\title{
Cardiac Arrhythmia Termination on the Vascular and Organ Scale
}

\author{
Dissertation \\ zur Erlangung des mathematisch-naturwissenschaftlichen \\ Doktorgrades \\ "Doctor rerum naturalium" \\ der Georg-August-Universität Göttingen \\ im Promotionsprogramm \\ Göttingen Graduate School for Neurosciences, Biophysics, and Molecular \\ Biosciences (GGNB) \\ der Georg-August University School of Science (GAUSS)
}

vorgelegt von

Daniel Hornung

aus Bonn

Göttingen, 2013 


\section{Betreuungsausschuss:}

Honorarprofessor Dr. Stefan Luther (Referent)

Forschungsgruppe Biomedizinische Physik, Max-Planck-Institut für Dynamik und Selbstorganisation, Göttingen

apl. Prof. Dr. Ulrich Parlitz

Forschungsgruppe Biomedizinische Physik, Max-Planck-Institut für Dynamik und Selbstorganisation, Göttingen

Prof. Dr. Eberhard Bodenschatz

Labor für Fluiddynamik, Strukturbildung und Biokomplexität, Max-Planck-Institut für Dynamik und Selbstorganisation, Göttingen

\section{Mitglieder der Prüfungskommission:}

Prof. Dr. Jörg Enderlein (Korreferent)

Forschungsgruppe Biophysik/Komplexe Systeme, Drittes Physikalisches Institut, GeorgAugust-Universität Göttingen

Dr. Eleni Katifori

Research Group Physics of Biological Organization, Max Planck Institute for Dynamics and Self-Organization, Göttingen

Prof. Dr. Andreas Janshoff

Institut für Physikalische Chemie, Georg-August-Universität Göttingen

Tag der mündlichen Prüfung: 26. November 2013 


Science is what we understand well enough to explain to a computer. Art is everything else we do.

We should continually be striving to transform every art into a science: in the process, we advance the art.

Donald Knuth $(\star 1938)$ 



\title{
GEORG-AUGUST-UNIVERSITÄT GÖTTINGEN
}

\author{
Fakultät für Physik
}

\section{Abstract}

\author{
by Daniel Hornung
}

Sudden cardiac death, caused by ventricular fibrillation, is a leading cause of mortality. To date, the only treatment is the delivery of a high-energy electrical shock through the heart, either externally or through implanted devices. Although potentially lifesaving, these shocks can be painful and traumatic for patients, especially when delivered due to diagnostic errors or device malfunctions. A promising new method to terminate ventricular fibrillation more gently is Low Energy Antifibrillation Pacing (LEAP). LEAP applies a series of pulses at low electric field strengths, thereby exciting the heart muscle locally at many different locations and synchronizing the tissue. In this work I show that this excitation takes place at the cardiac vasculature and that LEAP leads to substantial energy reductions. With the use of a micro-CT scanner, I obtained threedimensional data of cardiac vasculature of dogs and pigs and quantified the vessel sizes with a custom-developed algorithm. I found that the size distribution of the coronary vasculature follows a power law that can be transformed into a prediction of the dynamic behavior of cardiac tissue. To assess the efficiency of LEAP in clinically relevant settings, I performed in vivo and ex vivo experiments on porcine and canine hearts. On average, the defibrillation energy using LEAP could be decreased by up to $70 \%$ compared to the respective single shock energy. Pacing slower than the dominant fibrillatory frequency was more efficient than faster pacing, which supports the hypothesis that direct access to fibrillation vortex cores via heterogeneities is essential to LEAP success. 



\section{Contents}

Abstract vii

List of Figures $\quad$ xiii

List of Tables $\quad$ xvii

\begin{tabular}{ll}
\hline Abbreviations & xix
\end{tabular}

$\begin{array}{lll}1 & \text { Introduction } & 1\end{array}$

1.1 Excitable Media . . . . . . . . . . . . . . . . . . . . 2

1.1.1 The Heart and Arrhythmias . . . . . . . . . . . . . . . . . . 2

1.1 .2 Classical Defibrillation and a LEAP Forward . . . . . . . . . . . . . . . 2

1.2 Connecting Structure and Function . . . . . . . . . . . . . . . . . . . 3

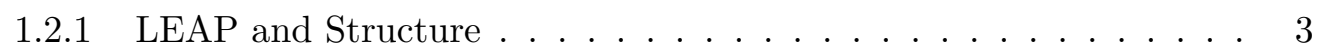

1.2 .2 Cardiac Vasculature . . . . . . . . . . . . . . . . 3

1.3 Classical and Advanced Defibrillation on Real Hearts . . . . . . . . . . . . 3

1.3.1 In Vivo and Ex Vivo Experiments . . . . . . . . . . . . . . . . . . . . . . 4

1.3 .2 Energy Reduction and LEAP Parameters . . . . . . . . . . . . . . 4

2 The Heart, an Excitable Medium 5

$2.1 \quad$ Excitable Media $\ldots \ldots \ldots \ldots \ldots \ldots \ldots$

2.1 .1 Examples of Excitable Media . . . . . . . . . . . . . . . 6

2.1 .2 Excitation and Wave Propagation in the Heart . . . . . . . . . . . 8

2.2 Modeling of Excitable Media . . . . . . . . . . . . . . . . . . . 10

2.2 .1 Bidomain and Monodomain Models . . . . . . . . . . . . . . . 11

$2.3 \quad$ Excitation Pathways in the Heart . . . . . . . . . . . . . . . . . 13

2.3.1 Overview over Cardiac Anatomy and Mechanic Function. . . . . . 13

$2.3 .2 \quad$ Biological Pacemakers . . . . . . . . . . . . . . . . . . 14

2.3 .3 From the Sinus to the AV Node. . . . . . . . . . . . . . . . . . . . . . . . . . . . 14

2.3 .4 Bundle of His and Purkinje System . . . . . . . . . . . . . . . 14

2.3 .5 Fiber Orientation . . . . . . . . . . . . . . . . . . . . . . . . . . . . . . . . . . 15

2.4 Pathological Heart Activations $\ldots \ldots \ldots \ldots$

2.4.1 AV Block: Time for a Backup Solution. . . . . . . . . . . . . . 15

$2.4 .2 \quad$ Alternans $\ldots \ldots \ldots \ldots \ldots \ldots \ldots$

2.4 .3 Ventricular Tachycardia: Mostly Harmless? . . . . . . . . . . . . . 17

$2.4 .4 \quad$ Ventricular Fibrillation $\ldots \ldots \ldots \ldots$ 


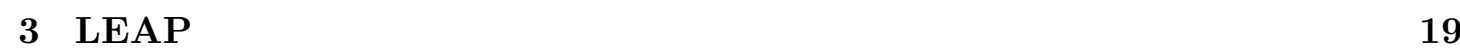

3.1 Termination of Arrhythmias . . . . . . . . . . . . . . . . . . . . . 19

3.2 External Fields and Tissue Boundaries . . . . . . . . . . . . . . . . . 20

3.3 Pinning of Spirals . . . . . . . . . . . . . . . . . . . . . 20

3.4 Low Energy Pacing . . . . . . . . . . . . . . . . . . . . . . . . . . . . . . . . . . . . . . . . . . . . . . .

$3.4 .1 \quad$ Fortified Spirals . . . . . . . . . . . . . . . . . . . . . . 21

3.4 .2 Obstacles to Assets . . . . . . . . . . . . . . . . . . . . . 21

$3.4 .3 \quad$ Many Small LEAPs to End Fibrillation . . . . . . . . . . . . . . . 22

\begin{tabular}{|lr|}
4 & Heterogeneities in Cardiac Tissue \\
\hline
\end{tabular}

4.1 Size Matters . . . . . . . . . . . . . . . . . . . . . . . . . 23

$4.1 .1 \quad$ Circular Heterogeneities Modeled . . . . . . . . . . . . . . . . . . 23

$4.1 .2 \quad$ Embedded Heterogeneities . . . . . . . . . . . . . . . . . . . 25

4.2 Heterogeneities in the Heart . . . . . . . . . . . . . . . . . . . . . . . . . . . . . 26

$4.2 .1 \quad$ Cardiac Vasculature . . . . . . . . . . . . . . . . . . . . . . 28

$4.2 .2 \quad$ Other Vascular Scaling Laws $\ldots \ldots \ldots$

$4.2 .3 \quad$ Size Distributions in the Literature . . . . . . . . . . . . . . . . 30

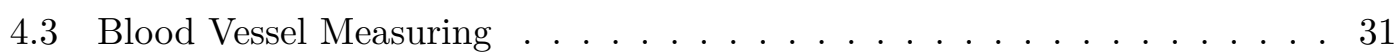

4.3 .1 Measurement methods . . . . . . . . . . . . . . . . . . 31

$4.3 .2 \quad \mu \mathrm{CT}$ scans $\ldots \ldots \ldots \ldots \ldots \ldots \ldots$

$4.3 .3 \quad$ Preparation and Scanning of Cardiac Tissue . . . . . . . . . . . . 34

4.4 Automatic Size Reconstruction . . . . . . . . . . . . . . . . . . . 36

$4.4 .1 \quad$ Locating the Vascular Tree $\ldots \ldots \ldots$. . . . . . . . . . 36

$4.4 .2 \quad$ Accurate Size Measurements on the Vascular Tree . . . . . . . . . 40

$4.4 .3 \quad$ Accuracy tests $\ldots \ldots \ldots \ldots \ldots$. . . . . . . . . . . . . . 42

4.5 Measuring the Size Distributions . . . . . . . . . . . . . . . . . . . . . . . . . . . . . . . . . . 43

$4.5 .1 \quad$ Data Acquisition of Dog Heart Vessel Sizes . . . . . . . . . . . 43

4.5 .2 Porcine Blood Vessel Sizes . . . . . . . . . . . . . . . . . . . . . . 44

4.6 Electrical Tissue Activation $\ldots \ldots \ldots \ldots$. . . . . . . . . . . . 47

4.6 .1 Activation Time Measurement . . . . . . . . . . . . . . . . . . 47

$4.6 .1 .1 \quad$ Optical Mapping of Cardiac Electrical Activity . . . . . . 48

4.6.1.2 The Experimental Setup of Optical Cardiac Activation Time Measurements . . . . . . . . . . . . . . . . . 49

$4.6 .1 .3 \quad$ Optical Mapping Image Analysis . . . . . . . . . . . . . . . 49

$4.6 .1 .4 \quad$ Activation Times in Cardiac Tissue . . . . . . . . . . . 51

4.6 .2 Linking Times and Sizes . . . . . . . . . . . . . . . . . 52

$\begin{array}{lll}5 & \text { Heterogeneity Characterization Results } & \mathbf{5 5}\end{array}$

5.1 Size Distributions of Blood Vessels . . . . . . . . . . . . . . . . . . . 55

5.1 .1 Canine Blood Vessel Size Distributions . . . . . . . . . . . . . . 55

5.1 .2 Porcine Blood Vessel Size Distributions . . . . . . . . . . . . . 57

5.1 .3 Vascular Size Distributions from Other Sources . . . . . . . . . . . 58

5.1 .4 Comparison of Vascular Size Distributions of Different Origin . . . 59

5.2 Activation Times . . . . . . . . . . . . . . . 60

$5.2 .0 \quad$ Activation Time Exponents $\ldots \ldots \ldots$. . . . . . . . . . 60

5.2 .1 Size Distributions Linked to Activation Times . . . . . . . . . . . . 60 
$\begin{array}{lll}6 & \text { In Vivo LEAP Experiments } & 63\end{array}$

6.1 Previous Studies . . . . . . . . . . . . . . . . . . . . . 63

6.2 Towards Clinical Application . . . . . . . . . . . . . . . . . . 64

$6.2 .1 \quad$ Experimental Criteria . . . . . . . . . . . . . . . . . . 64

6.2 .2 Clinically Relevant Animal Models for LEAP . . . . . . . . . . . . 65

6.3 The Experimental Setup . . . . . . . . . . . . . . . . . . 65

6.3 .1 Prototype LEAP Devices . . . . . . . . . . . . . . . 66

6.3.1.1 Power Amplifiers for Lower Energy Requirements and Higher Flexibility . . . . . . . . . . . . 67

$6.3 .1 .2 \quad$ A Custom-Built Defibrillator . . . . . . . . . . . . 68

6.3 .2 Electrical Connection to the Heart . . . . . . . . . . . . . . 71

6.3 .2 .1 A Temporarily Implanted Defibrillator. . . . . . . . . . 71

$6.3 .2 .2 \quad$ Epicardial Mesh Electrodes . . . . . . . . . . . . . . 71

6.3 .3 Physiological Measurement Recording . . . . . . . . . . . . . . 73

6.3 .4 The Animal Operation Room Setup . . . . . . . . . . . . . . . . . 74

6.4 Experimental Procedure . . . . . . . . . . . . . . . . . . . . . . . . . . . . . . . . . . 74

6.4 .1 Pre-Fibrillation . . . . . . . . . . . . . . . . . 76

$6.4 .1 .1 \quad$ ICD Phantom Implant $\ldots \ldots \ldots$. . . . . . . . . 76

6.4.1.2 Open Chest Electrode Application . . . . . . . . . . . . 77

6.4 .1 .3 Initial Impedance Measurement . . . . . . . . . . . . . 78

6.4 .2 Defibrillation to Evaluate LEAP Efficiency . . . . . . . . . . . . . 79

6.4 .2 .1 Choosing the Defibrillation Parameters . . . . . . . . . 80

6.4 .3 End of LEAP Experiment . . . . . . . . . . . . . . . . 82

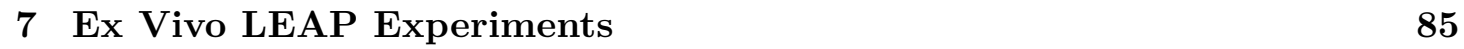

$7.1 \quad$ Benefits of Ex Vivo Antifibrillation Experiments . . . . . . . . . . . . . . 85

$7.1 .1 \quad$ A Controlled Environment . . . . . . . . . . . . . . . . . . . . . 85

7.1 .2 Experimental Efficiency $\ldots \ldots \ldots$. . . . . . . . . . . . . . . . . . . . . . . . . . . . . . . . .

7.1 .3 Contactless Activation Measurement . . . . . . . . . . . . . . . . . 86

7.2 Open Questions . . . . . . . . . . . . . . . . . . . . . . . 87

$7.2 .1 \quad$ Comparability with In Vivo Experiments . . . . . . . . . . . . . 87

7.2 .2 Comparison to other LEAP Evaluations . . . . . . . . . . . . . . . . . . . . . 87

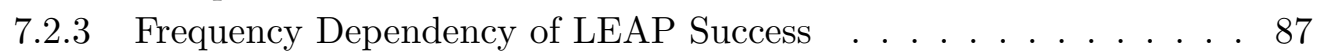

7.3 The Experimental Setup . . . . . . . . . . . . . . . . . . . . . . . . . . . . . . . . 88

$7.3 .1 \quad$ The Langendorff Perfusion System . . . . . . . . . . . . . . . . 88

7.3 .2 Optical Mapping . . . . . . . . . . . . . . . . . . . . . 90

7.3 .3 The Electrical Configuration . . . . . . . . . . . . . . . . . . . 90

7.3 .4 Effective Energy Transfer . . . . . . . . . . . . . . . . . . . . . . . . . . . . . . . . . . . . . . . . . . .

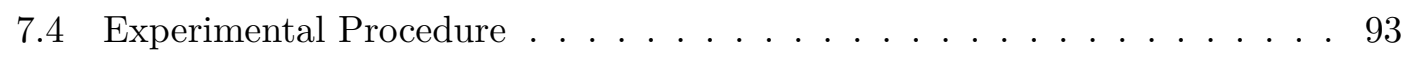

7.4 .1 Sample Preparation . . . . . . . . . . . . . . . . . . . . . 94

7.4 .2 Simulating a Physiological Environment . . . . . . . . . . . . . . . 94

7.4 .3 Defibrillation Energies . . . . . . . . . . . . . . . . . . . . . . . . . . . . . . . 95

7.4 .4 Frequency Scans . . . . . . . . . . . . . . . . . . . . . . . . . . . . . . . . . . . . . . . . .

7.5 End of Experiment $\ldots \ldots \ldots$. . . . . . . . . . . . . . . . . . . . . 97

\begin{tabular}{|ll}
8 & LEAP Experiment Results
\end{tabular}

$8.0 \quad$ On DFT Measurement . . . . . . . . . . . . . . . . . . . . . . . . . . 99 
8.1 Results of the In Vivo Experiments . . . . . . . . . . . . . . . . . . . . . . 101

$8.1 .1 \quad$ Evaluation of the Experimental Defibrillator Setup . . . . . . . . . 101

8.1 .2 Defibrillation Energies . . . . . . . . . . . . . . . . . . . . . 102

$8.1 .3 \quad$ LEAP Efficiency (In Vivo) . . . . . . . . . . . . . . . . . . 108

8.1 .4 Frequency Dependency of LEAP Success . . . . . . . . . . . . 110

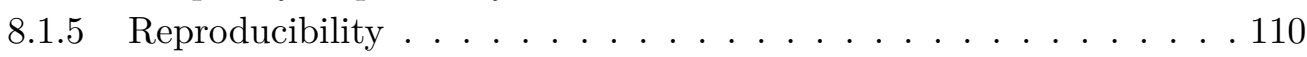

8.2 Results of the Ex Vivo Experiments . . . . . . . . . . . . . . . . . . . 113

$8.2 .1 \quad$ Setup Evaluation and Comparison to In Vivo . . . . . . . . . . . 113

$8.2 .2 \quad$ Defibrillation Energies . . . . . . . . . . . . . . . . . . . . . . . . . . . . . . . . . . . . . . . . .

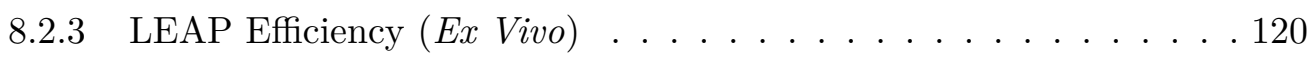

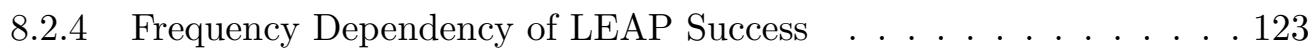

8.2 .5 Reliability of DFT Measurements . . . . . . . . . . . . . . . 123

$\begin{array}{lll}9 & \text { Discussion } & \mathbf{1 2 5}\end{array}$

9.1 Prediction of Activation from Vessel Sizes . . . . . . . . . . . . . . . . 125

9.2 Setup Evaluation . . . . . . . . . . . . . . . . . . . 126

$9.2 .1 \quad$ Clinical Relevance . . . . . . . . . . . . . . . . . . . 127

9.3 Defibrillation Energy Measurements . . . . . . . . . . . . . . . . . . . . . . . . . . . . 127

9.3 .1 The Unequal-Pairs Method . . . . . . . . . . . . . . . . . . . . 128

$9.3 .2 \quad$ DFT drift . . . . . . . . . . . . . . . . . . . . . . . . . . 128

9.4 Energy Reduction with LEAP . . . . . . . . . . . . . . . . . . . . . 129

9.5 Frequency Dependency of LEAP Success $\ldots \ldots$. . . . . . . . . . . . 130

10 Conclusion and Outlook 131

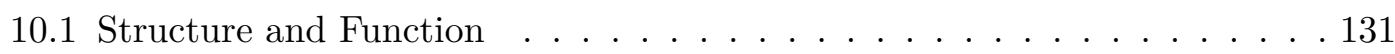

10.2 LEAP Energy . . . . . . . . . . . . . . . . . . . . . . . 131

10.3 LEAP Experiments . . . . . . . . . . . . . . . . . . . . 132

\begin{tabular}{ll}
\hline A Derivations & $\mathbf{1 3 5}$
\end{tabular}

A.1 Solution to the Bidomain Boundary Problem at Circular Boundaries . . . 135

A.2 Defibrillation threshold estimation . . . . . . . . . . . . . . . 137

A.2.1 Maximum Likelihood Estimation of a Defibrillation Probability . . 137

A.2.2 Error Estimation of the Defibrillation Probability. . . . . . . . . . 138

A.2.3 The "Unequal Pairs" Method . . . . . . . . . . . . . . . . . . . 138

A.2.3.1 Special Case: The Logistic Function . . . . . . . . . . . . . . . . . . . . . . . . . . . . . . . .

A.2.3.2 Different Energies . . . . . . . . . . . . . . . . 140

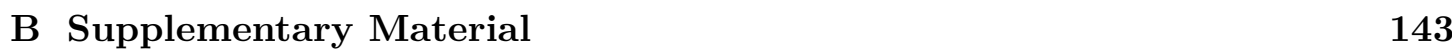

B.1 Vascular Size Distributions . . . . . . . . . . . . . . . . . . . . . . . . . . . . . . . . . . . . . . . . . . .

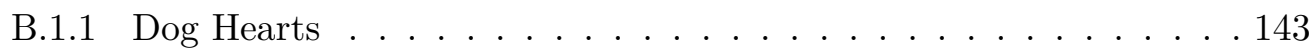

B.1.2 Pig Hearts. . . . . . . . . . . . . . . . . . . . . . . . . . . . . . . . 148

B.2 Frequency Dependency of LEAP Success . . . . . . . . . . . . . . . . . 151 


\section{List of Figures}

2.1 Excitable medium schematic . . . . . . . . . . . . . . . . . 6

2.2 Belousov-Zhabotinski Reactions, Cellular Automaton . . . . . . . . . . . . 7

2.3 Ion Channels $\ldots \ldots \ldots \ldots \ldots \ldots$

2.4 Action Potential of the Human Heart. . . . . . . . . . . . . . . . . . . . . . . . . . . .

2.5 Gap Junctions in Cardiomyocytes . . . . . . . . . . . . . . . . . . . 10

2.6 Simple Numerical Simulation $\ldots \ldots \ldots$

2.7 Restitution Curve . . . . . . . . . . . . . . . . . . . . . . . . . 16

$3.1 \quad$ ICD sketch $\ldots \ldots \ldots \ldots \ldots \ldots$

4.1 A Circular Obstacle . . . . . . . . . . . . . . . . . . . . . . . . 24

4.2 Depolarization at a Circular Obstacle . . . . . . . . . . . . . . 25

4.3 Endocardial Wall Structure . . . . . . . . . . . . . . . . . . . . 27

4.4 A Vascular Tree. . . . . . . . . . . . . . . . . . . . . . . . . . . . . . . . . . . . . . . 29

4.5 Vascular Exponents for Different Species . . . . . . . . . . . . . . . . . . . . . . . . . . . . . . 30

4.6 Blood Vessel Imaging. . . . . . . . . . . . . . . . . . . . . . . . . . . . 31

$4.7 \quad$ C T scan structure $\ldots \ldots \ldots \ldots . \ldots \ldots$

4.8 Example of Beam Hardening in a CT Image . . . . . . . . . . . . . . . . 34

$4.9 \quad \mu$ CT Scan Results $\ldots \ldots \ldots \ldots \ldots$

4.10 User-Assisted Vessel Reconstruction . . . . . . . . . . . . . . . . . . . . . . . . . . . . 37

4.11 Overview: Size Measurement Algorithm . . . . . . . . . . . . . . . . . . . 37

4.12 Skeletonization Process $\ldots \ldots \ldots$. . . . . . . . . . . . . . 38

4.13 Size Measurement from Cross Sections . . . . . . . . . . . . . . . . . . . . 41

4.14 Vascular Size Reconstruction - Graphical result . . . . . . . . . . . . . . . . . . 43

4.15 Size Measurement Calibration . . . . . . . . . . . . . . . . . . . . . . . . . . . . . . 44

$4.16 \mu \mathrm{CT}$ Cross Section through a Porcine Heart . . . . . . . . . . . . . 46

4.17 3D View of a Reconstructed Pig Heart . . . . . . . . . . . . . . . . . 46

4.18 The Minimum Obstacle Radius for Tissue Depolarization . . . . . . . . . 48

4.19 Optical Mapping Data . . . . . . . . . . . . . . . . . . . . . 50

4.20 Cardiac Tissue Activation Map . . . . . . . . . . . . . . . . . 51

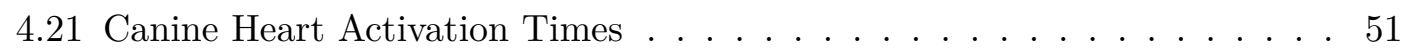

5.1 Vascular Size Distributions in Canine Ventricles and Atria . . . . . . . . . . 56

5.2 Scaling Exponents for Canine Ventricles and Atria Compared . . . . . . . 57

5.3 Size Scaling in a Porcine Heart . . . . . . . . . . . . . . . . . . 58

5.4 Size Scaling from Vascular Morphometric Data . . . . . . . . . . . . . . . 59

5.5 Vascular Size Exponents in Pig and Dogs Compared . . . . . . . . . . . . 60

$5.6 \quad$ Activation Time Exponents for Canine Ventricles and Atria Compared . . 61 
5.7 Vascular Size Distributions and Activation Times in Dog Hearts . . . . . 61

6.1 Power Amplifiers for LEAP Defibrillation . . . . . . . . . . . . . . . . . . 66

6.2 Power Amplifiers for defibrillation shocks . . . . . . . . . . . . . . . . 68

6.3 Experimental LEAP Defibrillator . . . . . . . . . . . . . . . . . . . . . . . . . . . . . . . . . . . . . . . . . . . .

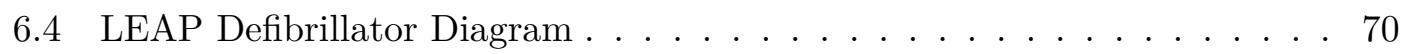

6.5 Defibrillation Data $\ldots \ldots \ldots \ldots \ldots \ldots$. . . . . . . . . . . . . . 71

6.6 ICD Catheter and ICD Can Phantom . . . . . . . . . . . . . 72

6.7 Mesh Electrodes . . . . . . . . . . . . . . . . . . . . . . 72

6.9 ICD Catheter Position in the RV . . . . . . . . . . . . . . . . 76

6.10 In Vivo Pig Heart Action Potential . . . . . . . . . . . . . . . . . . . . 77

6.11 Patch Mesh Electrodes on the Heart . . . . . . . . . . . . . . . . . . 78

6.12 Schematic of the in vivo experimental protocol. . . . . . . . . . . . . . . . 80

6.13 In Vivo Experiment duration . . . . . . . . . . . . . . . . . . . 83

7.1 Perfusion Setup Outline $\ldots \ldots \ldots$. . . . . . . . . . . . . . . . . . . . . . . . . . . . 88

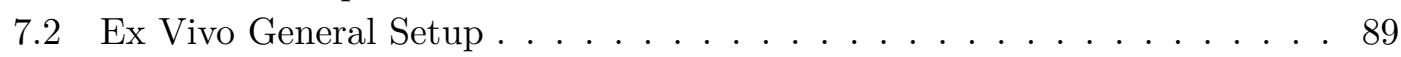

7.3 Langendorff Perfusion $\ldots \ldots \ldots$. . . . . . . . . . . . . . . . . . . . 89

7.4 Perfusion Bath: Electrical Configuration . . . . . . . . . . . . . . . . . 91

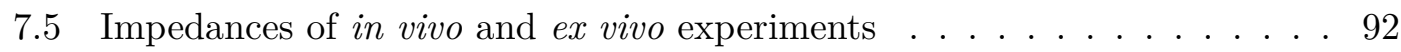

7.6 Ex Vivo Experiment duration $\ldots \ldots \ldots$. . . . . . . . . . . . . . . 95

$8.1 \quad$ In vivo energies . . . . . . . . . . . . . . . . . . . . . . . . . . . . . . . 102

8.2 Pig in vivo defibrillation energies, overview . . . . . . . . . . . . . . . . 104

8.3 Pig in vivo defibrillation energies, overview . . . . . . . . . . . . . . . . . . . . . . . . . . . . . . . . . . . .

8.4 Dog in vivo defibrillation energies, overview . . . . . . . . . . . . . . . . . 106

8.5 Dog in vivo defibrillation energies, overview . . . . . . . . . . . . . . . . . 107

8.6 Estimated in vivo defibrillation energies . . . . . . . . . . . . . . . . . . 109

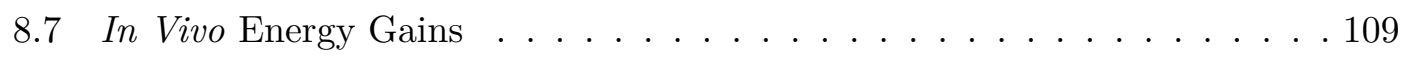

8.8 In vivo frequency dependency of LEAP efficiency . . . . . . . . . . . . . . 111

8.9 Pig ex vivo defibrillation energies overview, wedge preparation. . . . . . . . 114

8.10 Pig ex vivo defibrillation energies overview, wedge preparation. . . . . . . . 115

8.11 Pig ex vivo defibrillation energies overview, whole heart . . . . . . . . . . . . 116

8.12 Pig ex vivo defibrillation energies overview, whole heart . . . . . . . . . . . 117

8.13 Dog ex vivo defibrillation energies overview . . . . . . . . . . . . . . . 118

8.14 Dog ex vivo defibrillation energies overview . . . . . . . . . . . . . . . . 119

8.15 Estimated Ex Vivo Defibrillation Energies . . . . . . . . . . . . . . . . . . 121

8.16 Ex Vivo Energy Gains . . . . . . . . . . . . . . . . . . . . . . . . . . . . . 121

8.17 Ex vivo frequency dependency of LEAP efficiency . . . . . . . . . . . . . 122

9.1 Comparison of Field Geometries ～. . . . . . . . . . . . . . . . 126

A.1 Measurement probability for the Unequal Pairs method . . . . . . . . . . 139

A.2 Measurement probability with $E, E+\Delta \ldots \ldots$. . . . . . . . . . . 140

B.1 Vascular Size Distributions in Canine Atria . . . . . . . . . . . . . . . . . 143

B.6 Vascular Size Distributions in Canine Ventricles . . . . . . . . . . . . . . . 146

B.9 Vascular Size Distributions in Canine Atria . . . . . . . . . . . . . . . . . 148

B.10 Vascular Size Distributions in Canine Atria . . . . . . . . . . . . . . . . 149 
B.11 Vascular Size Distributions in Canine Atria . . . . . . . . . . . . . . . . . 149

B.12 Vascular Size Distributions in Canine Atria . . . . . . . . . . . . . . . . 150

B.13 Vascular Size Distributions in Canine Atria . . . . . . . . . . . . . . . . 150

B.14 LEAP Success for Different Pacing Frequencies . . . . . . . . . . . . . . 151 



\section{List of Tables}

4.1 X-ray mass attenuation coefficients $\ldots \ldots \ldots \ldots$

$5.1 \quad$ Size Distribution Exponents for Dogs . . . . . . . . . . . . . . . . . . 56

5.2 Activation Time Exponents for Dogs . . . . . . . . . . . . . . . . . . . 62

6.1 Overview over defibrillation shock wave forms. . . . . . . . . . . . . . . 81

7.1 In vivo and ex vivo impedances . . . . . . . . . . . . . . . . . . . . . . . . . . . . . . . 92

7.2 Cardioplegia Solution Composition . . . . . . . . . . . . . . . . . . . . . . . . . . . . 94

7.3 Solution Compositions . . . . . . . . . . . . . . . . . . . 95

$9.1 \quad$ LEAP energies in vivo and ex vivo . . . . . . . . . . . . . . . . . . 127 



\section{Abbreviations}

$\begin{array}{ll}\text { ATP } & \text { Anti-Tachycardia Pacing } \\ \text { APD } & \text { Action Potential Duration } \\ \text { BZ }(\text { R }) & \text { Belousov-Zhabotinski (Reaction) } \\ \text { cAMP } & \text { cyclic Adenosine MonoPhosphate } \\ \text { CT } & \text { Computed Tomography } \\ \text { DFT } & \text { Defibrillation Threshold } \\ \text { ECG } & \text { Electocardiogram } \\ \text { FFT } & \text { Fast Fourier Transform } \\ \text { HU } & \text { Hounsfield Unit } \\ \text { ICD } & \text { Implantable Cardioverter-Defibrillator } \\ \text { LAD } & \text { Left Anterior Descending artery } \\ \text { LCx } & \text { Left Circumflex artery } \\ \text { LEAP } & \text { Low Energy Antifibrillation Pacing } \\ \text { LV } & \text { Left Ventricle } \\ \text { MAP } & \text { Monophasic Action Potential } \\ \text { MIP } & \text { Maximum Intensity Projection } \\ \text { MRI } & \text { Magnetic Resonance Imaging } \\ \text { RCA } & \text { Right Coronary Artery } \\ \text { RV } & \text { Right Ventricle } \\ \text { SA node } & \text { Sino-Atrial node } \\ \text { VF } & \text { Ventricular Fibrillation } \\ \text { VT } & \text { Ventricular Tachycardia } \\ \text { WEH } & \text { Wave Emitting Heterogeneity } \\ \end{array}$





\section{Chapter 1}

\section{Introduction}

The history of modern physics started in the $16^{\text {th }}$ and $17^{\text {th }}$ century with the discovery and mathematically exact description of natural phenomena on the largest length scales imaginable at that time: Earth, the Moon, the Sun and the other planets in our solar system. The classical mechanical laws by Kepler, Galileo and Newton describing those systems remained valid for hundreds of years and most scientific discoveries were concerned with macroscopic systems. Mechanics, thermodynamics and electricity could be described easily on everyday scales. The scientific (or at least the physical) world view changed again at the beginning of the $20^{\text {th }}$ century, with the revolutionary insight that it was not possible within the known natural laws to take the limit towards very large or very small systems. One can say that the most important physical advancements in most of the $20^{\text {th }}$ century were on the (sub)atomic and the cosmological scale.

But in the last decades, a new class of problems has received the focus: complex systems on the boundary of statistical physics, biology, information theory, socio-economic sciences and nonlinear dynamics. They tend to happen on mesoscopic scales and show emergent behavior (both phrases have become too much buzzwords to be of much use anymore) and so far proved very resilient against complete analytical descriptions. Incidentally this probably also is as accurate a definition of "complex systems" as one can get. Bar-Yam [1] gives a very similar definition: "the complexity of a system is the amount of information needed in order to describe it." From a physicist's point of view, also biology and medicine are complex systems and questions arising in these fields should be tackled with physical methods for thorough understanding.

One of the leading causes of mortality worldwide is sudden cardiac death (SCD). In Europe alone, an estimated 500000 people per year [2] die because their heart "stops beating". Globally in the industrialized countries, the incidence of SCD is approximately 
40-100 cases per 100000 inhabitants and year [2-4] ${ }^{1}$. It turns out the reason for ventricular fibrillation, the medical condition underlying SCD is best understood by describing the heart as an excitable medium, a prime example of complex systems. With this description one can gain insight into the underlying mechanisms and develop fundamentally better treatments. In the following chapters, I will show how heterogeneities in the heart act with the surroundings tissue when synchronizing it with low energy electric stimuli and that low-energy approaches increase the defibrillation efficiency by $70 \%$.

\section{$1.1 \quad$ Excitable Media}

Many active systems far from equilibrium can be described as excitable media. In chapter 2 I show how many biological and chemical systems and also cellular automata share the same mechanism of activation, refractory periods and communication which is common to this class of systems. For many systems, modeling as an excitable medium offers a practical abstraction.

\subsubsection{The Heart and Arrhythmias}

The heart and especially the cardiac muscle is a classical example for an excitable medium since excitation may spread over the whole muscle once activated in one place. There are many mathematical models [7] to describe its behavior through reactiondiffusion equations, ranging from just two local variables (for example in the manner of [8] or [9]) to models trying to model cellular dynamics to much higher degrees of accuracy (e.g. [10] with more than 40 variables).

Given the complexity of the underlying dynamics, it comes as no surprise that the heart supports several spatio-temporal excitation patterns. The sinus rhythm during normal heart beat is just one example, but there is also a whole zoo of arrhythmias, some of which are self-sustaining and potentially lethal.

\subsubsection{Classical Defibrillation and a LEAP Forward}

The classical method to terminate arrhythmias, if local stimulation with low voltages is ineffective, is to deliver large electrical shocks across the heart. This effectively resets all cells of the heart into a common state, thereby eliminating spatial activity inhomogeneities. Starting with the next heart beat, the normal heart rhythm can take over

\footnotetext{
${ }^{1}$ For SCD numbers in e.g. India, a prototypical newly industrialized country, see [5, 6].
} 
again. Patients with a disposition to life-threatening arrhythmias may obtain implanted defibrillators to automatically deliver these shocks if required.

Because the defibrillation shocks may be potentially harmful to the tissue and can be perceived as very painful, better arrhythmia-terminating methods are required. In chapter 3. I present an approach towards more gentle defibrillation, Low-Energy Antifibrillation Pacing (LEAP), where many small shocks reset the cardiac muscle locally at many places.

\subsection{Connecting Structure and Function}

\subsubsection{LEAP and Structure}

The mechanism by which LEAP causes synchronization locally at many locations depends on structural heterogeneities in the tissue. Ventricular tachycardia and fibrillation, the most dangerous kinds of arrhythmias are also influenced by the heart's internal structures, which suggests an intimate connection between the mechanisms of the arrhythmias and LEAP.

\subsubsection{Cardiac Vasculature}

The most ubiquitous structuring component in the heart is the vasculature, which makes it an ideal candidate for LEAP's mechanism. I show in chapter 4 and 5 that the blood vessels' size distribution follows a power law and that this distribution can be linked conclusively to the effects of low electric fields on heart tissue as demonstrated in optical mapping experiments.

\subsection{Classical and Advanced Defibrillation on Real Hearts}

Although numerical models can predict many properties of (de)fibrillation, complete and accurate simulation of a whole heart and the effect of electric fields is not possible yet. Until then and to verify numerical results, experimental tests are necessary and provide new insights. 


\subsubsection{In Vivo and Ex Vivo Experiments}

Testing defibrillation on whole hearts or regular tissue is closer to eventual application in humans than experiments on in vitro cell cultures. In the chapters 6 and 7, I describe in vivo and ex vivo experiments to evaluate the implementation of LEAP in clinically relevant environments. While in vivo experiments are as close to the human organism as possible in pre-clinical studies, ex vivo setups provide for a more controlled environment. Combined they maximize the time of experiment for each heart.

\subsubsection{Energy Reduction and LEAP Parameters}

Although the experimental defibrillator devices used in the experiments are far from being reduced to a size that can be implanted, they show that LEAP has the potential to lower the defibrillation energy below the pain threshold.

In the experiments we evaluated the energy gain which is possible using LEAP and found that it is already substantial even on first tries. We also varied pacing parameters in order to determine if LEAP efficacy can be attributed mainly to successive synchronization of cardiac tissue or to stimulation at the "centers" of fibrillation. The answers to these questions are given in chapter 8 and 9 . 


\section{Chapter 2}

\section{The Heart, an Excitable Medium}

The heart as the most active muscle in the human body is a paradigmatic example for a self-organized system of a special class: an excitable medium. In the healthy heart, the initial contraction signal is given from few specialized pacemaker cells. The excitation propagates through the heart muscle itself, reaching its farthest corners before the heart relaxes, only to be activated again a few hundred milliseconds later. Yet many excitable media have the ability to exhibit self-sustained spatio-temporal activity which does not need external driving. In the heart these activities show up as several kinds of arrhythmias, some of which can be severely life threatening.

This chapter gives an introduction to what excitable media are, some examples and what could go wrong for otherwise comparatively reliable systems such as the heart.

\subsection{Excitable Media}

One of the features setting living matter apart from dead are sustained chemical reactions which take place far away from equilibrium. This abundance of energy makes way for a class of systems which are generally characterized as excitable media ${ }^{1}$.

An excitable medium has two specific properties: Its elements have a state with specific properties which may change over time (governing the local dynamics) and the current state can be communicated to the elements' neighbors (adding one or more spatial dimensions to the dynamics). Elements in an excitable state may, through interaction with neighboring elements, become excited and in this state, excite their neighbors in turn. Following this state, they drop into some refractory state before they become excitable again. Figure 2.1 shows a schematic view of this principle.

\footnotetext{
${ }^{1}$ See for example [1] for an overview over common properties of excitable media.
} 


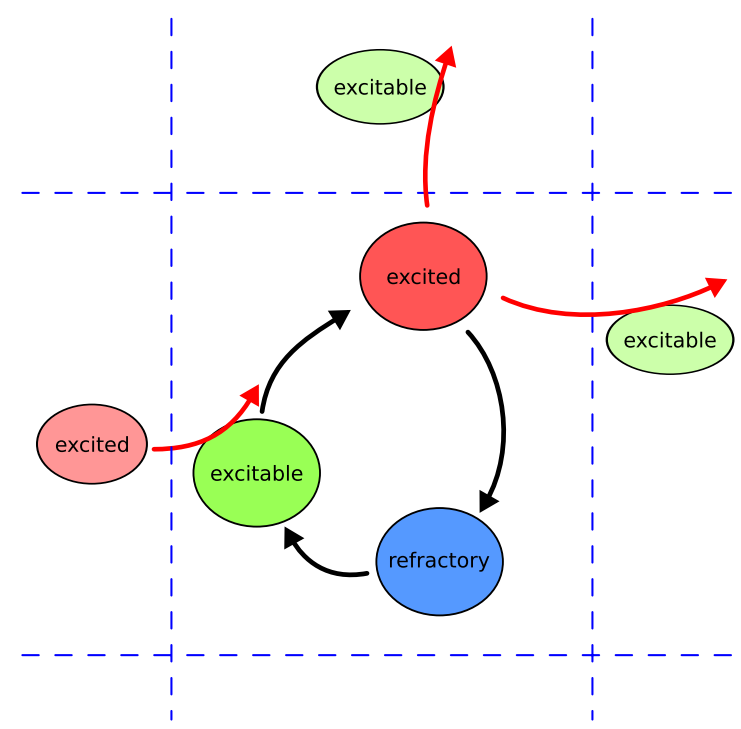

Figure 2.1: Schematic view of an excitable medium: Each element has a state and excited elements may push their excitable neighbors into the excited state.

Of course, discrete states as in this simplified description are rare, most systems' states $\mathbf{y}$ are continuous, e.g. molecular concentrations in biological tissue. The local dynamical behavior $\mathbf{y}(t)$ can then mostly be described by a set of differential equations:

$$
\frac{\partial \mathbf{y}}{\partial t}=\mathbf{F}(\mathbf{y}, t)
$$

Spatial coupling in the medium is usually modeled by adding a diffusive term to the system of local equations, resulting in the form of a so-called reaction-diffusion system:

$$
\frac{\partial y_{i}(\mathbf{x})}{\partial t}=F_{i}(\mathbf{y}(\mathbf{x}), t)+\nabla \mathbf{D}_{i}(\mathbf{x}) \nabla y_{i}(\mathbf{x})
$$

It has been known for a long time [12] that excitable media can feature wave propagation and even reentrant patterns such as spiral waves which survive prolonged times without external stimulation. Figure 2.6 shows examples of such wave patterns in numerical simulations.

\subsubsection{Examples of Excitable Media}

Among the best-known and oldest published reaction-diffusion systems are the BelousovZhabotinski reactions (BZR) [13, 14]. In this class of inorganic cyclic chemical reactions, malonic acid is oxidized by bromate, catalyzed by cerium or similar ions. The current (oxidation) state of the medium can be made visible by indicators such as ferroin, changing color between blue and red. A stirred BZ medium shows cyclic changes of the color, while in resting medium circular or spiral waves (in two dimensions) and even scroll 

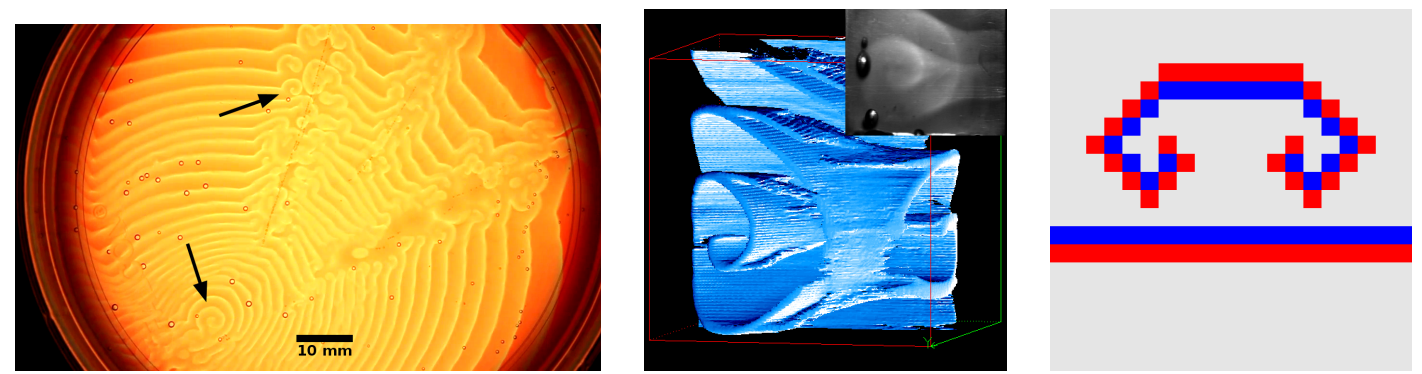

Figure 2.2: Left and middle: Images of BZR (2D and 3D). In the leftmost image, spontaneous circular activity and spiral waves (arrows) can be observed. The image in the middle shows a rendering of reconstructed three-dimensional scroll waves in a BZR medium as described in [16, with a corresponding raw image as inset. Images courtesy of Claudia Richter, Marion Kunze, Göttingen (2D) and Patricia Dähmlow, Magdeburg (3D) Right: Cellular automaton simulation of a plane wave traveling from top to bottom, with a double spiral induced in the wave back. The snapshot was made four time steps after the spiral was induced by manually setting a few pixels in the wave back to the activated state.

waves for the three-dimensional case [15, 16] can develop (see figure 2.2, left and middle).

The simplest numerical model for excitable media are cellular automata where each cell can switch between at least three states, as shown in figure 2.1. A set of transition rules $\mu: x_{i}(t) \times S_{i}(t) \mapsto x_{i}(t+1)$ which modify the current state $x_{i}$ of each cell $i$ depending on the neighborhood $S_{i}$ could look like this:

\begin{tabular}{l|l||l}
$x_{i}(t)$ & $S_{i}(t)$ & $x_{i}(t+1)$ \\
\hline excitable & no excited cell & excitable \\
excitable & at least one excited cell & excited \\
excited & irrelevant & refractory \\
refractory & irrelevant & excitable
\end{tabular}

This rule set was implemented using the cellular automata simulation software Golly [17, one exemplary result can be seen in figure 2.2 (right). Cellular automata like this have been employed for over 60 years [18, 19] as simplified representations of spreading excitation in neuronal tissue.

One especially high-level example of excitation waves with discrete "cells" can be observed in sports audiences, where human beings act as elements of the excitable medium: Fans in a stadium may synchronize to form a so-called "Mexican Wave", where a wave of cheering goes through the audience and around the playing field or at least parts of it. Farkas et al., Farkas and Vicsek [20, 21] have proposed a detailed model to describe the dynamics of spectators reacting on the cheering of their neighbors.

Currently one of the most studied excitable media from biology are colonies of the social slime mold amoebae Dictyostelium discoideum [22]. As long as there is sufficient 
food supply, i.e. bacteria to feed on, the D. discoideum population grows by mitosis. In contrast, under starvation the D. discoideum cells aggregate to migrate as multicellular "slugs", form a fruiting body and finally release some cells in the form of spores which may start this cycle again. The aggregation is organized by periodic production and emission of a messenger molecule, cyclic adenosine monophosphate (cAMP). Single starving D. discoideum cells follow the cAMP gradient via chemotaxis, thus building the clusters necessary for the migration and spore building part of their life cycle.

Phosphodiesterase leads to a constantly decreasing cAMP concentration through hydrolysis inside and outside the cells, unless more cAMP is synthesized or secreted, respectively. Outside the cells, cAMP concentration also changes due to diffusion. cAMP synthesis inside D. discoideum depends on the local cAMP concentration outside but is limited by the current sensitivity of the cAMP sensors which become desensitized after prolonged exposure 23]. Tyson et al. [24] were able to put this into a system of differential equations corresponding to $(2.2)$ which describe the evolution of the intracellular and extracellular cAMP concentrations $\beta$ and $\gamma$ and the fraction of sensitive cAMP receptors in the membrane $\rho$ :

$$
\begin{aligned}
& \frac{\partial \gamma}{\partial t}=k_{t} \frac{V_{\mathrm{ext}}}{V_{\mathrm{int}}} \beta-k_{\mathrm{ext}} \gamma+D \Delta \gamma \\
& \frac{\partial \beta}{\partial t}=F(\rho, \gamma)-k_{t} \beta-k_{\mathrm{int}} \beta \\
& \frac{\partial \rho}{\partial t}=-f_{1}(\gamma) \rho-f_{2}(\gamma)(1-\rho)
\end{aligned}
$$

Here, $k_{t}, k_{\text {ext }}$ and $k_{\text {int }}$ describe the cAMP secretion and hydrolysis rates, $D$ is the diffusion coefficient and $F, f_{1}$ and $f_{2}$ describe the production rate and sensor (de)activation in more detail.

With adequate parameters, this model can exhibit circular and spiral waves of changing cAMP concentration with periods of a few minutes, just as can be found in homogeneously distributed D. discoideum populations.

\subsubsection{Excitation and Wave Propagation in the Heart}

In the heart, muscle cells (cardiomyocytes) have to fulfill a complicated task: On an external stimulus they must contract in a synchronized way and end this synchronization again after some time, waiting for the next stimulus to arrive. Electrical activation and mechanical action of the cardiomyocytes is communicated via changing ion concentrations in the intra- and extracellular space. 


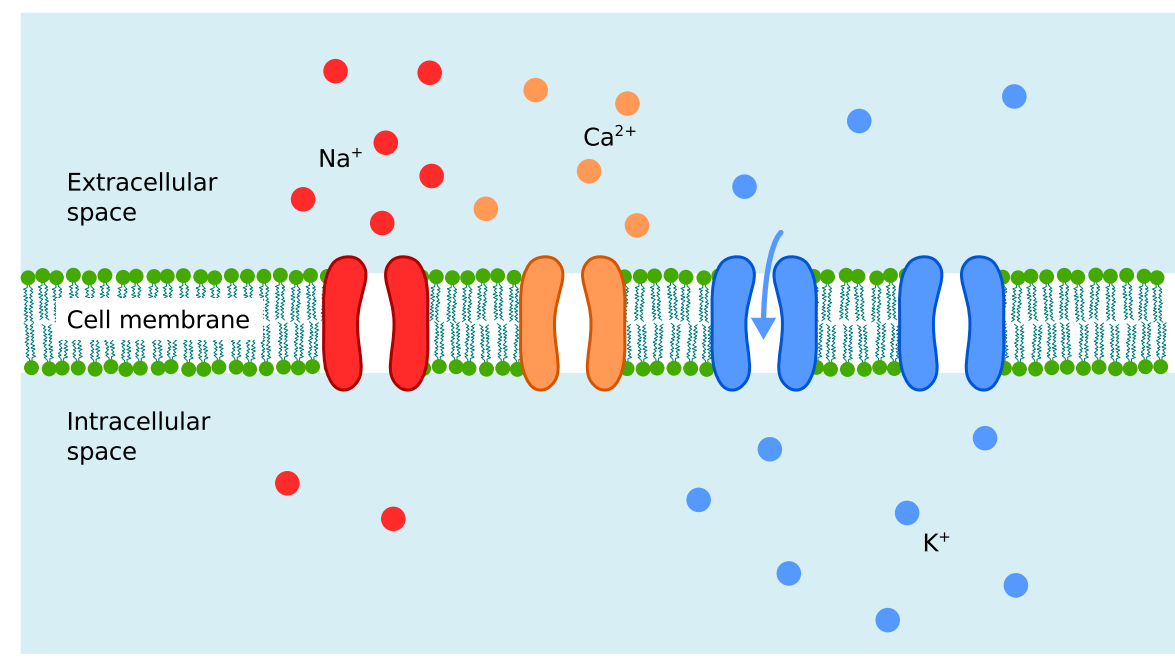

Figure 2.3: A selection of ion channels in the membrane of a cardiomyocyte. From left to right: (Fast) $\mathrm{Na}^{+}$channel, (slow) $\mathrm{Ca}^{2+}$ channel, $\mathrm{K}^{+}$inward rectifying channel, $\mathrm{K}^{+}$voltage gated channel.

In the heart, there is a strong surplus of $\mathrm{Na}^{+}$and $\mathrm{Ca}^{2+}$ ions outside and $\mathrm{K}^{+}$ions inside the cardiomyocytes. Ion channels allow some ions to diffuse between intra- and extracellular space. The ion concentration gradient is kept mostly constant by selective ion pumps in the cell membrane, which leads to an electrical potential difference across the membrane, the membrane potential. For resting cardiomyocytes, the membrane potential is usually between -90 and $-85 \mathrm{mV}$. Some of the ion channels are not static but their conductivity is dependent on the membrane potential or specific ion or molecule concentrations. For example the actual contraction of cardiomyocytes is induced by an increase of the intracellular $\mathrm{Ca}^{2+}$ concentration, an effect that is amplified by calcium sensitive calcium channels in the sarcoplasmic reticulum, an intracellular calcium storing structure.

Once the membrane potential of a cardiomyocyte rises above a certain threshold due to external activation, $\mathrm{Na}^{+}$channels open and let more $\mathrm{Na}^{+}$flow into the cell, increasing the membrane potential even more. These fast sodium channels are only open for a few milliseconds, but the depolarization is kept at a high level by the opening of slower calcium channels. At the same time the constant outflux of potassium ions into the extracellular medium is decreased and only at the end of the activation phase, 200 to $400 \mathrm{~ms}$ after the onset, additional $\mathrm{K}^{+}$channels open to restore the original membrane potential; the cell membrane returns to the polarized state. The action potential, the change of the membrane potential during activation and deactivation, is shown in figure 2.4

While the membrane potential increases noticeably during activation of a cardiomyocyte, it should be noted that the ion concentrations stay virtually constant since already small 


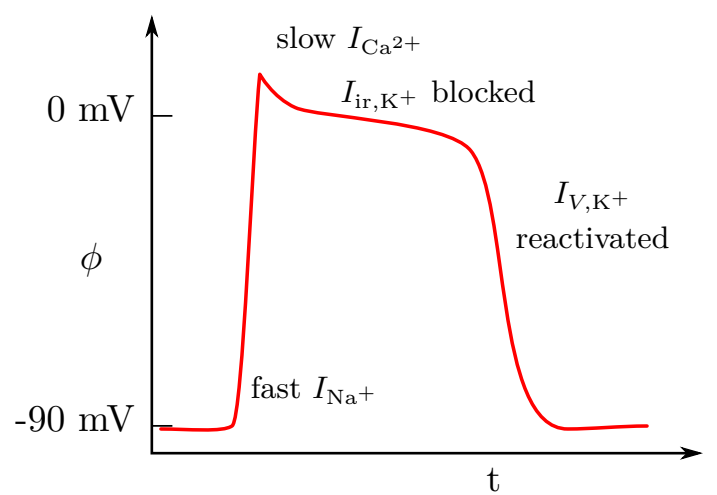

Figure 2.4: A schematic of the human cardiac action potential. Its shape emerge from the interaction of the cardiac ion channels. After the fast $\mathrm{Na}^{+}$channels initiate the upstroke, the plateau phase is maintained by slower $\mathrm{Ca}^{2+}$ channels and the blocking of inward rectifying $\mathrm{K}^{+}$channels. At the end of the action potential, additional $\mathrm{K}^{+}$ channels open to guarantee quick repolarization.

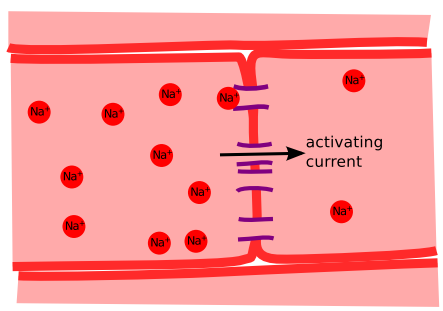

FiguRE 2.5: Gap junctions (in violet) connect neighboring cardiomyocytes and thus allow direct communication of action potentials, mainly in fiber direction.

charges ares sufficient for significant voltage changes.

The action potential in the heart spreads to neighboring cells through the extracellular space, but also directly from cell to cell. Cardiomyocytes are elongated cells of 50 to $70 \mu \mathrm{m}$ length, their cytoplasm is connected directly to their neighbors through gap junctions, mainly at the ends (figure 2.5). This anisotropy leads to significant differences in the excitation propagation velocities along and perpendicular to the muscle fiber orientations.

The described mechanisms, excitation amplification, depolarization and later repolarization and relaying this excitation to neighbors cause the spread of excitation as waves in cardiac tissue. In the section 'Excitation Pathways in the Heart]', I will explain the excitation of the whole heart in more detail and section 'Pathological Heart Activations" will cover pathological activation patterns.

\subsection{Modeling of Excitable Media}

Considering the medical importance of a functioning heart, it is understandable that extensive efforts have been made to describe the functional behavior of the cardiomyocytes 
in varying levels of detail and complexity. Mathematical models allow to understand the mechanisms underlying cardiac behavior, in particular pathologic activation patterns and methods to terminate them. Here I will focus on the electrical aspects, the equally important electro-mechanical coupling is beyond the scope of this work.

\subsubsection{Bidomain and Monodomain Models}

In a first approximation, it makes sense to consider mainly the electric potential on the inside and outside of cardiac muscle cells. On a macroscopic level, one can make the assumption that both the intracellular and extracellular domain pervade the whole geometric space, i.e. the state of each point consists of the intra- and extracellular state at that point.

This idea can be formulated in the bidomain model of cardiac tissue:

$j_{\mathrm{i}}$ and $j_{\mathrm{e}}$ are the current densities within the intra- and extracellular space, respectively. These current densities are governed by the potentials $\phi$. and conductivities $\sigma$. (conductivity tensors in the case of anisotropic media):

$$
\begin{aligned}
& j_{\mathrm{i}}=-\sigma_{\mathrm{i}} \nabla \phi_{\mathrm{i}} \\
& j_{\mathrm{e}}=-\sigma_{\mathrm{e}} \nabla \phi_{\mathrm{e}}
\end{aligned}
$$

Interaction between the two domains is mediated through the transmembrane current density $j_{\mathrm{m}}$, which scales with the surface to volume ratio $\chi$ :

$$
\nabla \sigma_{\mathrm{i}} \nabla \phi_{\mathrm{i}}=\chi j_{\mathrm{m}}=-\nabla \sigma_{\mathrm{e}} \nabla \phi_{\mathrm{e}}
$$

If the intracellular domain does not cover the same physical space as the extracellular domain, no-flux boundary conditions for the intracellular medium are assumed on the boundary (see figure 4.1, boundary effects are discussed in more detail in section 4.1 'Circular Heterogeneities Modeled'):

$$
\left.\mathbf{n}_{\mathrm{i}} \cdot \nabla \phi_{\mathrm{i}}\right|_{\partial \Omega_{i}}=0
$$

The extracellular medium's potential and current are assumed to be identical on both sides of this boundary.

Introducing the (trans)membrane potential $\phi_{\mathrm{m}}=\phi_{\mathrm{i}}-\phi_{\mathrm{e}}$ and the membrane capacitance per area $C_{\mathrm{m}}, j_{\mathrm{m}}$ can also be written as:

$$
j_{\mathrm{m}}=C_{\mathrm{m}} \frac{\partial \phi_{\mathrm{m}}}{\partial t}+j_{\mathrm{ion}}\left(\phi_{\mathrm{m}}\right)
$$


Here the ionic membrane current $j_{\text {ion }}$ includes the linear and nonlinear effects that make up the cells' behavior. In most models the overall transmembrane current is composed of currents for different types of ion channels and pumps, many of which depend not only resistively on the membrane voltage but also on the internal state of the cell.

Usually the previous equations are rewritten in a slightly different way which is closer to typical numerical approaches to solve them:

$$
\begin{aligned}
\frac{\partial \phi_{\mathrm{m}}}{\partial t} & =-\frac{1}{C_{\mathrm{m}}}\left(\frac{1}{\chi} \nabla \sigma_{\mathrm{e}} \nabla \phi_{\mathrm{e}}+j_{\mathrm{ion}}\left(\phi_{\mathrm{m}}\right)\right) \\
\nabla\left(\sigma_{\mathrm{e}}+\sigma_{\mathrm{i}}\right) \nabla \phi_{\mathrm{e}} & =-\nabla \sigma_{\mathrm{i}} \nabla \phi_{\mathrm{m}}
\end{aligned}
$$

The equations (2.11) and 2.12 constitute the bidomain equations. The most common way to solve such a bidomain system is for each time step to first integrate 2.11) and then solve (2.12) for $\phi_{\mathrm{e}}$.

Since especially the latter step is computationally expensive for large systems, it makes sense to consider the special case where $\sigma_{\mathrm{e}}$ and $\sigma_{\mathrm{i}}$ are not independent of each other, but are only scaled differently as in [25]:

$$
\sigma_{\mathrm{i}}=k \sigma_{\mathrm{e}}
$$

Putting (2.13) and $\phi_{\mathrm{i}}=\phi_{\mathrm{m}}+\phi_{\mathrm{e}}$ into (2.8) yields:

$$
\begin{aligned}
\nabla \sigma_{\mathrm{e}} \nabla \phi_{\mathrm{e}} & =-k \nabla \sigma_{\mathrm{e}} \nabla\left(\phi_{\mathrm{m}}+\phi_{\mathrm{e}}\right) \\
& =-\frac{k}{k+1} \nabla \sigma_{\mathrm{e}} \nabla \phi_{\mathrm{m}} \\
\stackrel{\partial \phi_{\mathrm{m}}}{\partial t} & =\frac{1}{C_{\mathrm{m}}}\left(\frac{1}{\chi} \frac{k}{k+1} \nabla \sigma_{\mathrm{e}} \nabla \phi_{\mathrm{m}}-j_{\mathrm{ion}}\left(\phi_{\mathrm{m}}\right)\right)
\end{aligned}
$$

Equation (2.16), the monodomain equation, depends only on the membrane potential $\phi_{\mathrm{m}}$ which makes solving it numerically a lot simpler. Although the assumption 2.13 does not hold physiologically in many cases, the monodomain representation can still reproduce a broad range of physiological behavior qualitatively and quantitatively.

The mono- and bidomain equations are compatible with the formal requirements for a reaction-diffusion system (equation (2.2) ) with just one or two diffusive variables. Additional variables $\mathbf{u}$ are "hidden" in the ion current dynamics of $j_{\text {ion }}=j_{\text {ion }}\left(\phi_{m}(\mathbf{x}), \mathbf{u}(\mathbf{x})\right)$ and evolve locally according to some $\frac{\partial \mathbf{u}(\mathbf{x})}{\partial t}=\mathbf{f}(\phi(\mathbf{x}), \mathbf{u}(\mathbf{x}))$.

Together with boundary and initial conditions and a suitable $\mathbf{f}$, the mono- and bidomain equations can describe the evolution of an excitable medium which is continuously 

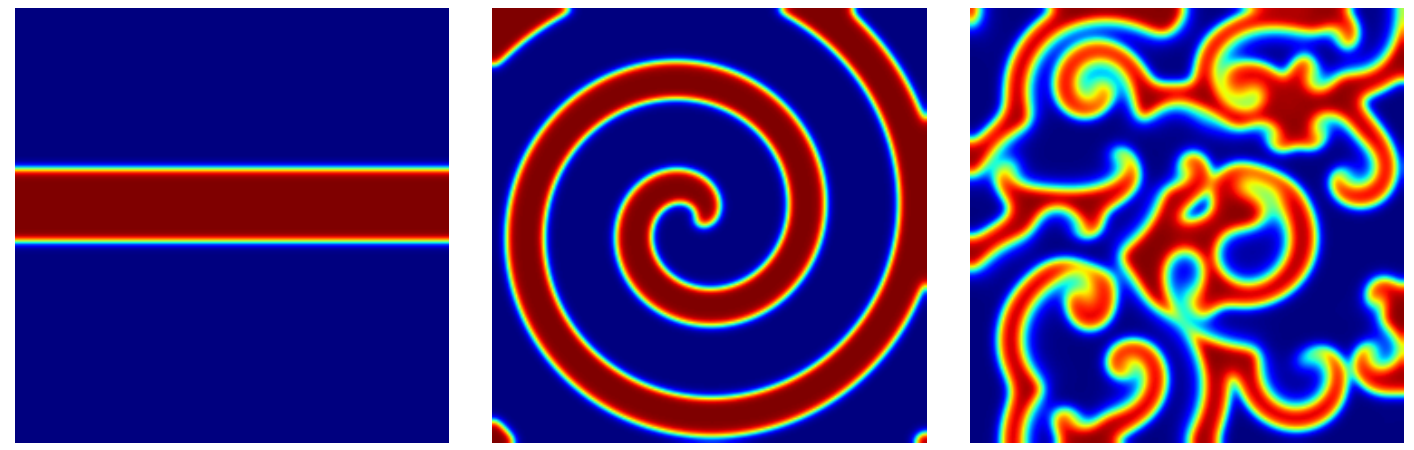

FiguRE 2.6: Numerical integration of the Barkley model on a square domain. The color, from blue to red, indicates the value of the activation variable. Left: A plane wave traveling in upward direction across the tissue Middle: A self-sustained spiral, in clockwise rotation. Right: Turbulent activation in the Bär-Eiswirth model[26], a modified Barkley model with an additional nonlinear term. The model systems were integrated using the MediaSim software framework (main author: Philip Bittihn).

extended in space. Several methods exist to handle spatially extended systems numerically, most of them discretize space so that the system only needs to be calculated at a finite number of sites. Typical examples of a particularly simple model (as proposed by Barkley [9]) system are shown in figure 2.6.

\subsection{Excitation Pathways in the Heart}

Abnormal heart rhythms can be serious and even fatal diseases, but usually the heart beats about 100000 times a day without problems. Therefore it is crucial to have a thorough understanding of how normal cardiac excitation spreads in the heart before investigating pathological patterns.

\subsubsection{Overview over Cardiac Anatomy and Mechanic Function}

The heart is "optimized" to supply the body with a steady flow of oxygenated blood of approximately 5 to $20 \frac{1}{\mathrm{~min}}$ (this and other basic physiological data are taken from [27]). To meet this requirement, the heart is structured into four main compartments, right and left atria and ventricles, each atrium connects to its corresponding ventricle.

During each cardiac cycle, at the beginning of the ventricular systole, the ventricles contract, and the increasing pressure of approximately $120 \mathrm{mmHg}$ (in the left ventricle) ejects about half the intraventricular blood volume into the respective arteries, which lead into the lung (right ventricle) or via the aorta (left ventricle) into the body. Valves prevent the blood from flowing back from the ventricles into the atria during and from the arteries into the ventricles after the contraction. The beginning of ventricular relaxation 
marks the end of the systole and start of diastole. The aortic and pulmonary valves close to prevent the reflow of blood into the ventricles while the comparatively elastic pulmonary artery and especially the aorta keep up the pressure at a high level (about $80 \mathrm{mmHg}$ in the aorta).

The ventricular relaxation in the diastolic phase lets blood flow into the ventricles from the atria, which shortly contract at the end of the diastole, just before the next systole begins.

In contrast to the left ventricle (LV), the right ventricle (RV) has a systolic pressure of only about $25 \mathrm{mmHg}$.

\subsubsection{Biological Pacemakers}

Electrical activation in the heart is started by specialized pacemaker cells in the sinoatrial node (SA node) in the right atrium (RA). These cells spontaneously oscillate between polarization and depolarization and in this way initiate excitation in connected excitation pathways. If for some reason electrical activity is not initiated in the SA node or blocked, other cells may act as backup pacemakers, e.g. in the atrioventricular node (AV node) or in the ventricles themselves. To prevent accidental pulse initiation in these secondary and tertiary pacemakers, their normal oscillation rate is only about 40 and 30 beats /min, respectively. During normal sinus rhythm, they are overdriven and reset by the standard activation.

\subsubsection{From the Sinus to the AV Node}

From the SA node, the excitation spreads over the atria, whose contraction denotes the end of the diastolic phase. The ventricles are electrically insulated from the atria so that they cannot be directly activated. The only connection is via the AV node and the bundle of His, the AV node is located in the RA above the interventricular septum, the bundle of His continues from the AV node in two branches into the septum. Once an excitation wave activates the AV node, activation does not propagate immediately into the bundle of His, but is delayed by about 0.1 to $0.2 \mathrm{~s}$. This permits the atria to contract completely and relax again before the ventricles start contracting.

\subsubsection{Bundle of His and Purkinje System}

To maximize blood pumping efficiency, ventricular contraction is synchronized as much as possible. For this purpose, the excitation signal in the ventricles is first conducted 
by the bundle of His in two branches in the interventricular septum and then further distributed by a branched system of Purkinje fibers. In this specialized conduction system, the propagation velocity in is increased approximately threefold compared to the normal myocardium.

According to the endpoints of the Purkinje fiber system, normal electrical activation in the ventricle starts approximately at the apex (the most inferior point, which is furthest from the arterial valves) and continues to the base. Contraction continues until about $0.1 \mathrm{~s}$ after the whole ventricle has been activated.

\subsubsection{Fiber Orientation}

Cardiomyocytes are elongated cells with a width to length ratio of about 5:1. In a review by Valderrábano [28], it is pointed out that the anisotropy of the conductivity tensor $\sigma$ is caused mainly by the particular orientation of the cardiomyocytes. The bulk of the resistance is due to the interfaces between cells, and e.g. gap junctions are mainly located at the ends of cardiomyocytes.

The ventricular cardiomyocytes are arranged in thin sheets of a few cells thickness which make up the cardiac muscle structure. In each of these sheets, the single cells are typically ordered parallel to each other with a preferred direction, winding around the heart. In [29], Holzapfel and Ogden give further insight into this multi level structure, which mainly serves optimal contraction of the cardiac muscle.

As a result of the hierarchical orientation structure, wave propagation in the ventricles along the main fiber orientation is about twice as fast as in a perpendicular direction.

\subsection{Pathological Heart Activations}

Considering the heart's activation and pumping mechanism is very robust, that the majority of people live for many decades without major cardiac malfunction, but still pathological cardiac excitation patterns may develop. The consequences can range from being not even noticeable to potentially lethal.

\subsubsection{AV Block: Time for a Backup Solution}

If for reasons such as inflammation or local ischemia the AV node or bundle of His is adversely affected, normal conduction of the activation signal from the atria to the 


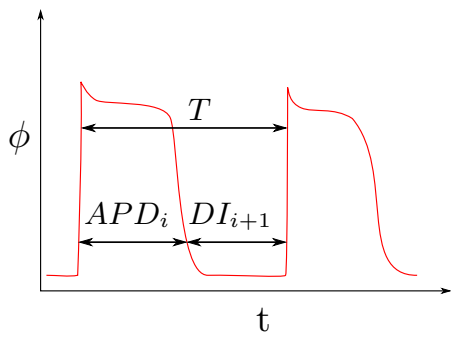

(a) APD and DI

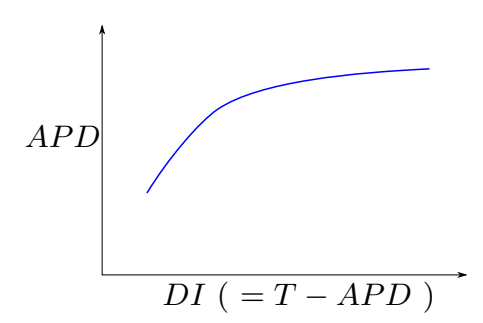

(b) Restitution curve

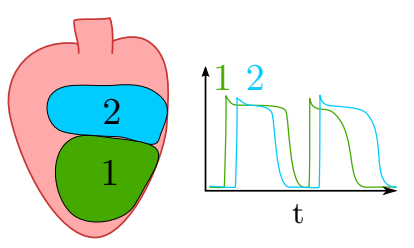

(c) Discordant alternans

FIGURE 2.7: Schematic APD restitution curve. (a) The beat-to-beat interval is identified by $T$, the action potential duration (for example when $\phi>\phi_{0}+0.2\left(\phi_{\max }-\phi_{0}\right)$ ) by $A P D$ and the remaining diastolic interval by $D I$. (b) Restitution curves are a simplified way to describe the dependence of an action potential on the time since the last action potential. Here an APD-DI restitution curve is used, if the slope is larger than 1 at $A P D(D I)=T-D I$, there is no single stable fixed point. (c) Spatially discordant alternans with two regions, which alternately exhibit short and long action potentials.

Figure according to 31 .

ventricle may be inhibited. In these cases, as noted in 2.3.2, further pacemaker sites take over to initiate contraction of the ventricle.

The resulting (ventricular) heart beat is significantly slower than the normal sinus rhythm, typically less than 40 beats / min [30], because higher frequencies of the ventricular pacemaker cells might interfere with the normal sinus rhythm.

\subsubsection{Alternans}

In some $\operatorname{cases}^{2}$, rapid heart beat from the sinoatrial node can lead to variations in the response of the ventricular action potential. This effect, called alternans, can be intuitively understood by considering the restitution curve which describes in the manner of an iterated map how the action potential duration (APD) depends on the preceding diastolic interval (DI) (figure 2.7).

If $\frac{\mathrm{d} A P D}{\mathrm{~d} D I}>1$ at $A P D(D I)=T-D I$, there is not a single limit cycle when the system is being paced (from the SA node or by external pacing), but possibly alternans (a case of period doubling) can develop. In the heart this may lead to regions of alternating behavior (figure 2.7c). Because media in this regime is prone to more complex behavior and patients whose ECG displays such alternans are in higher risk of potentially fatal arrhythmias, occurrence of alternans is an important cardiological marker in clinical practice [31.

\footnotetext{
${ }^{2}$ For example if one of the branches of the bundle of His or parts of the Purkinje fiber system is blocked.
} 


\subsubsection{Ventricular Tachycardia: Mostly Harmless?}

If for some reason, for example due to anatomical reentry (an excitation wave reaches medium which is excitable again through a unidirectional pathway) or from irregular depolarizations, excitation spreads in the ventricle before it is fully excitable, self-sustained spiraling excitation patterns may emerge, similarly to the simulation result in the middle image of figure 2.6. Because the spiral rotation frequency is a lot faster than the pulses entering through the AV node, the spiral is not influenced by whatever rhythm the atrium provides.

At about 150 beats/min, this arrhythmia still makes the heart contract sufficiently to supply the body and brain with oxygen. But although such ventricular tachycardia (VT) is not immediately fatal, it can degenerate into ventricular fibrillation (VF) if it is sustained and therefore constitutes a considerable threat.

\subsubsection{Ventricular Fibrillation}

Although the frequency of ventricular tachycardia is drastically increased as compared to normal sinus rhythm, it is regular and synchronized across the ventricle so that the heart can still contract. Prolonged episodes of VT however carry the risk that this regularity is lost and that the synchronized spiral breaks up into turbulent activity. In this state of ventricular fibrillation, different regions of the cardiac muscle are contracting in an uncorrelated manner at different times, so that the net pumping power vanishes. Unless treated immediately, the resulting loss of oxygen supply leads to irrecoverable and eventually lethal brain damage within minutes.

If fibrillation occurs not in the ventricles but in the atria, the low conduction velocity in the AV node effectively acts as a low pass filter and prevents propagation of the fast stimuli into the ventricles.

Several mechanisms may underlie VF, the most prominent being the "mother rotor" and wandering wavelets. In the case of a stable mother rotor spiral wave, distant regions can not follow the spiral frequency, causing waves to break up. Alternatively, the medium's dynamic may allow the continuous formation and annihilation of excitation wavelets. Both mechanism can occur in the same medium [32]. 



\section{Chapter 3}

\section{LEAP}

\subsection{Termination of Arrhythmias}

Cardiac arrhythmias can be terminated by shocks of high current which flow through the myocardium. The current forces all myocytes into excitation, after the shock the tissue is generally refractory, preventing further propagation of arrhythmic activity. After this refractory state, the next normal sinus rhythm signal can take over again.

Clinically this shock is either applied transthoracically through a pair of electrodes attached to the skin or, for patients with high risk of dangerous arrhythmias, automatically by an implanted device. These implantable cardioverter-defibrillators (ICD) consist of a metallic case which is usually located between the rib cage and the left pectoralis muscle and and a catheter in the right ventricle. The catheter is fitted with a helical coil which serves as one electrode, the ICD case is the other electrode across which defibrillatory shocks can be delivered. See figure 3.1 for a sketch of the electric field and figure $6.6 \mathrm{a}$ for a view of the catheter.

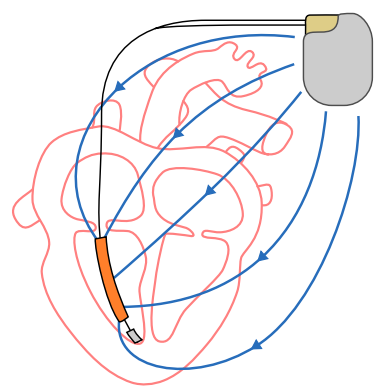

FIGURE 3.1: Electric field geometry between the case of an implantable cardioverterdefibrillator and the catheter inside the right ventricle of the heart. 


\subsection{External Fields and Tissue Boundaries}

Obviously electric fields have effects on the dynamics of heart tissue, understanding these effects allows to develop insights into the mechanisms of defibrillation and to develop more efficient defibrillation methods. Sobie et al. [33] and Tung [34] showed that both diverging electric fields and changes in the tissue conductivities can lead to local depolarization and hyperpolarization of cardiac tissue. Fishler [35] added the relative density of cardiomycoytes to the list of depolarization causes. The depolarization at tissue heterogeneities can, depending on shape and size of the heterogeneity and on the electric field strength, act as a nucleation core for new excitation waves. At minimally effective field strengths, only the largest heterogeneities (typically the tissue boundaries) emit waves, whereas if the electric field is at or above the defibrillation field strength (about $6 \frac{\mathrm{V}}{\mathrm{cm}}[36]$ ) the membrane potential of the whole tissue is elevated above the excitation threshold.

Details about the interaction of electric fields with heterogeneities are given in more detail in section 4.1 (Size Matters).

\subsection{Pinning of Spirals}

In numerical models as well as in reality, spiral excitation may move on complex paths (see [7] for an overview), the spiral tip's trajectory generally also depends on the structure of the underlying tissue. Most significant in this context are heterogeneities which are not excitable themselves, rotating spirals may attach to these obstacles and enter a periodic steady state. Experimentally this has been observed for example at the base of the papillary muscle [32].

\subsection{Low Energy Pacing}

Although defibrillation by external or internal defibrillators (ICDs) is a life-saving measure in most cases, especially ICDs are burdened by a number of drawbacks: The high energy associated with defibrillation shocks may be detrimental to the affected tissue [37. Maybe more significant yet are the psychological effects. Several studies [38-40] have found significant negative effects of delivered shocks on the quality of life of patients, this finding may well be connected to the fact that about $10 \%$ of all ICD patients experience inappropriately delivered shocks within the first 12 months after implantation [41. For some patients [42, the experience of ICD shocks can be traumatic enough 
to rather have the ICD explanted or deactivated than suffering another shock in spite of the often life-saving effects.

It is important to note that modern ICDs do not deliver the full shock energy in all cases. When the patient only suffers from monomorphic VT, it is often sufficient to stimulate the heart locally from a small bipolar electrode at the tip of the intraventricular catheter with a frequency higher than that of the tachycardia. Such anti-tachycardia pacing (ATP) is commonly sufficient to drive the self-sustained spiral center into the boundaries of the tissue (similar to an effect described in [43]). The energies involved in ATP only need to be sufficient to excite the myocardium locally. Only if ATP fails or is unlikely to succeed, for example in the case of VF, the full defibrillation shock is delivered.

If it were possible to decrease the energy required to terminate even complex VF below the perceived pain threshold, this would mean a tremendous gain in the quality of life for ICD patients and probably also increase their physical health. Fortunately there are physical principles by which such a reduction could be achieved.

\subsubsection{Fortified Spirals}

ATP is successful against freely rotating spirals because of the annihilating properties of waves in excitable media, which allow the faster of two periodic wave sources to entrain gradually larger regions until the whole medium is synchronized. If a spiral is pinned to an unexcitable obstacle however, this mechanism may fail if the pacing source is sufficiently far away from the obstacle: although the spiral tip is unpinned and driven away, the pacing wavefront creates a new pinned free end [44].

\subsubsection{Obstacles to Assets}

The idea of low-energy antifibrillation pacing (LEAP) is to utilize the very tissue heterogeneities which inhibit ATP's success. While unpinning and driving away of pinned spiral is not possible when the pacing source is far away, it is feasible for pacing sources in the vicinity (relative to the spiral wavelength) of the obstacle [45, 46]. Obviously with a single intraventricular catheter, it is impossible to guarantee that local pacing always occurs from very close to the relevant obstacle(s). In 1999 however, Pumir and Krinsky [47] showed that it is possible to combine the necessity of potentially many pacing sites with the specifically required locations: by applying an external electric field $\mathbf{E}$, heterogeneities, including those with a pinned spiral, emit excitation waves, which can lead to the unpinning and thereby the potential removal of formerly pinned spirals. The energy 
required for this activation of obstacles as wave emission sites should be about a factor of 100 lower than for defibrillation.

\subsubsection{Many Small LEAPs to End Fibrillation}

One important restriction for LEAP success is the angle or phase of the spiral relative to the electric field at the instant of pacing. Only if the "unpinning window" in the phase is hit, a wave can be emitted and unpin the spiral [47, 48]. In order to increase the chance of unpinning and thus LEAP success, a series of pulses set apart by $T_{\Omega \Omega} \neq T_{\circledast}$ are delivered to perform a phase scan over the spectrum of different spiral phases. With $T_{\Omega \Omega}<T_{\circledast}$ (named overdrive pacing), unpinned spirals will be driven away further from the former pinning center.

However because even pulses which fail to unpin the spiral influence its phase, overdrive pinning can lead to phase locking [49], so that underdrive pacing with $T_{\Omega \Omega}>T_{\Im}$ may be more successful. These two contradicting strategies up to now remained to be tested experimentally (some promising results could already be demonstrated in [45]).

A crucial advantage of LEAP is that it should be comparatively simple to implement clinically without major changes currently implanted ICDs. Possibly it may even be sufficient to just adapt the antifibrillation software to deliver a series of LEAP pulses instead of a single high energy shock. If LEAP fails, maybe because it terminates VF only with a finite probability, a traditional single defibrillation shock could follow. 


\section{Chapter 4}

\section{Heterogeneities in Cardiac Tissue}

As briefly explained in section 3.2 (External Fields and Tissue Boundaries), external fields can lead to the emission of activation waves at tissue boundaries. This heterogeneity effect can to a certain extent also take place in structures where the medium properties change significantly, although it is most prominent at tissue boundaries.

Both size and shape of the heterogeneities play an important role at determining the effect an electric field has on the tissue. In this chapter I will focus on one type of heterogeneity which is comparatively easy to quantify and nearly ubiquitous in the myocardium, on blood vessels.

\subsection{Size Matters}

\subsubsection{Circular Heterogeneities Modeled}

To quantify the idea of boundaries as excitation sources as discussed in section 3.2 . consider a circular heterogeneity of radius $R$ in an isotropic medium. Outside the cardiac tissue, we assume that the extracellular properties are the same as inside $\left(\phi_{\mathrm{e}}=\phi_{\mathrm{o}}\right.$, $\sigma_{\mathrm{e}}=\sigma_{\mathrm{o}}$ ), while the missing or dead cardiomyocytes are modeled by infinite intracellular resistance, leading to no-flux boundary conditions for the intracellular current. This, together with charge conservation for currents across the boundary (with boundary normal $\mathbf{n}$ ) in the extracellular medium, can be formulated as

$$
\begin{aligned}
& \mathbf{n} \cdot \sigma_{\mathrm{e}} \nabla \phi_{\mathrm{e}}=\mathbf{n} \cdot \sigma_{\mathrm{o}} \nabla \phi_{\mathrm{o}} \\
& \mathbf{n} \cdot \sigma_{\mathrm{i}} \nabla \phi_{\mathrm{i}}=0,
\end{aligned}
$$




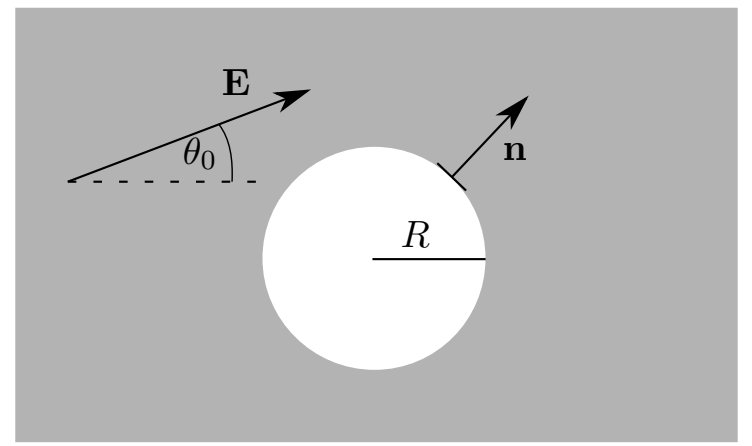

FIGURE 4.1: Sketch of a circular obstacle with radius $R$ inside an otherwise continuous medium. The surface normal is $\mathbf{n}$, the external electric field $\mathbf{E}$ forms an angle of $\theta_{0}$ to the $\mathbf{x}$ axis.

where $\phi_{\mathrm{o}}$ is the outside potential. Assuming a constant electric field $\mathbf{E}=-\nabla \phi_{\mathrm{o}}$ and the simpler case of an isotropic medium, we can combine the two equations to:

$$
\mathbf{n} \cdot \nabla \phi_{\mathrm{m}}=\mathbf{n} \cdot \mathbf{E}
$$

For small deviations $\phi$ of the membrane potential from the resting potential, $\phi_{\mathrm{m}}=$ $\phi_{\text {resting }}+\phi$, the transmembrane ionic current can be linearized so that the effective transmembrane resistance (at $\phi_{\mathrm{m}} \approx \phi_{\text {resting}}$ ) is sufficient to describe the system.

In the steady state, this means that the monodomain equation (2.16) and the boundary condition 4.3 can be rewritten as follows:

$$
\begin{aligned}
\Delta \phi-\frac{1}{\lambda^{2}} \phi & =0 \\
\mathbf{n} \cdot(\nabla \phi-\mathbf{E}) & =0
\end{aligned}
$$

Here $\lambda$ is the electrotonic space constant governing the decay of point potential sources over a distance, see the appendix A.1 for more details.

For the two-dimensional case of circular heterogeneities of radius $R$ embedded into a medium (see figure 4.1), these equations can be solved in polar coordinates $(r, \theta)$ by modified Bessel functions of the second kind [47]:

$$
\phi(r, \theta)=-\lambda|\mathbf{E}| \frac{K_{1}\left(\frac{r}{\lambda}\right)}{K_{1}^{\prime}\left(\frac{R}{\lambda}\right)} \cos \left(\theta-\theta_{0}\right)
$$

This solution leads to hyperpolarization at the cathodal and depolarization at the anodal side of the obstacle, with an effect proportional to the field strength. The exact 


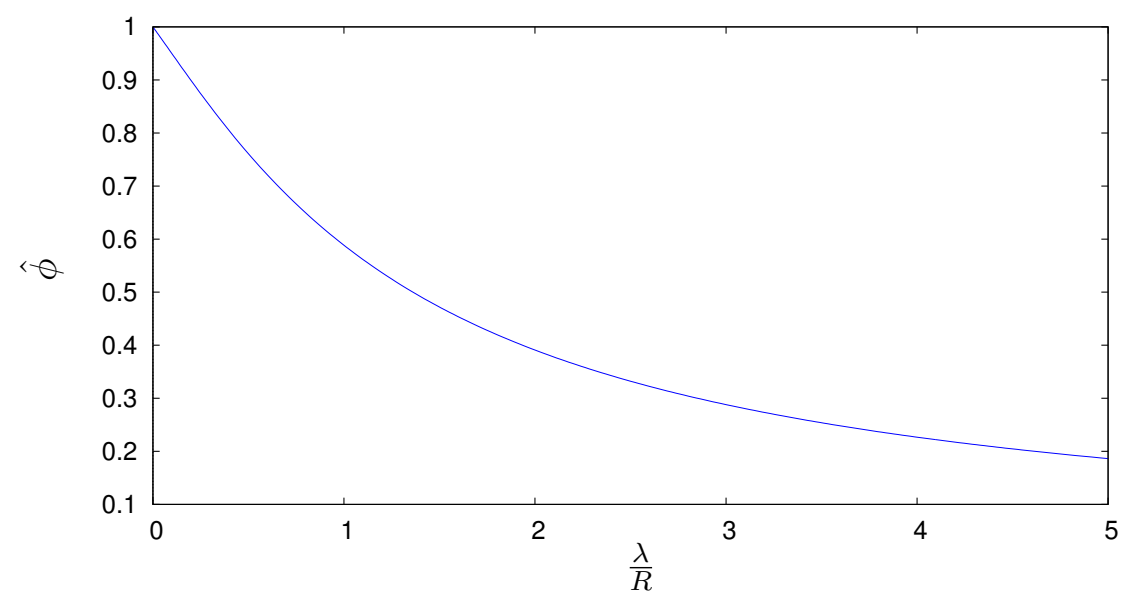

FIGURE 4.2: The normalized maximum depolarization $\hat{\phi}(R)=\frac{1}{\lambda|\mathbf{E}|} \phi\left(r, \theta_{0}\right)$ for given normalized positive curvatures $\frac{\lambda}{R}$.

dependency of the depolarization on the obstacle size is plotted in figure 4.2. One easily sees that for a given electric field, there is a critical obstacle size required to reach the threshold potential $\phi_{\text {thr }}$ which is required for activation of the cardiomyocytes. All obstacles of this size or larger will push cardiomyocytes into the excited state and thus serve as wave emitting heterogeneities (WEH).

Building upon these findings, Bittihn et al. [50] developed a more general theory of the interaction of boundaries and applied electric field. It describes how the shape and especially the curvature of the interface determines tissue depolarization. One implication is that pieces of cardiac tissue embedded into non-cardiac surroundings also experience activation depending on their size and that this effect can even outperform that of non-cardiac enclosures. These results are detailed in the appendix section A.1.

\subsubsection{Embedded Heterogeneities}

In the heart of course, there is not just one single heterogeneity, but whole structures which can be influenced by electric fields. For the moment we assume they can be modeled as spatially discrete obstacles $o_{i}$ with sizes much smaller than the distances between neighboring obstacles. Then for an applied electric field of a given strength, some of these obstacles emit spherical (or, in the case of a homogeneously anisotropic medium, elliptical) excitation waves which spread until the whole medium has been activated.

These heterogeneities which are embedded into the excitable medium are expected to play a crucial role both in the genesis and the sustainment of turbulent spatio-temporal activation patterns and in the termination thereof: 
- The presence of heterogeneities in an otherwise homogeneous medium can lead to wave breakup and therefore turbulent behavior [51 53 .

- Spiral or scroll waves (in two or three dimensions, respectively) tend to pin to heterogeneities in the medium, which may also stabilize the turbulent state against plane waves trying to synchronize the medium [44, 54, 55].

- In contrast to the previous two points, heterogeneities may also serve as wave emitting sites and thereby help to terminate fibrillation-like turbulent states [45, 47.

\subsection{Heterogeneities in the Heart}

In cardiac tissue, heterogeneities of many types and geometries exist. Some are boundaries where the myocardium and fluids or other tissue types meet, while others are formed by more or less subtle changes of physiological properties.

Among the first kind are:

- The epicardial (outside of the heart) boundary of the heart: The heart is embedded into a flui filled sac, the pericardium [56], which protects the heart and eases its movements by providing lubrication. The epicardium as a heterogeneity has a negative boundary curvature as described in section A.1 in the appendix.

- The endocardial (inside of the heart) side of the heart: While the epicardium is comparatively smooth, this is not the case for the endocardium. To support the heart's blood pumping, it is structured in a complicated way, with different characteristics in the atria and the ventricles. Some of the endocardial atrial walls have muscular links, the pectinate muscles. Similarly, the ventricles are strengthened on their interior by columnar muscles, the trabecular muscles which run along the ventricular walls. Additionally the ventricles have papillary muscles, column-like muscles which are attached to the inferior ventricle walls at one end and prevent the atrial valves (connected by the chordae tendineae, fibrous strings) from opening into the atria during the systolic phase. Figure 4.3 illustrates the anatomical features of the ventricular and atrial endocardium.

While the overall curvature of the interior cavities of the heart (atria and ventricles) is of course positive, the complicated structure at smaller scales prevents the assignment of a single typical size which would describe the behavior completely. 


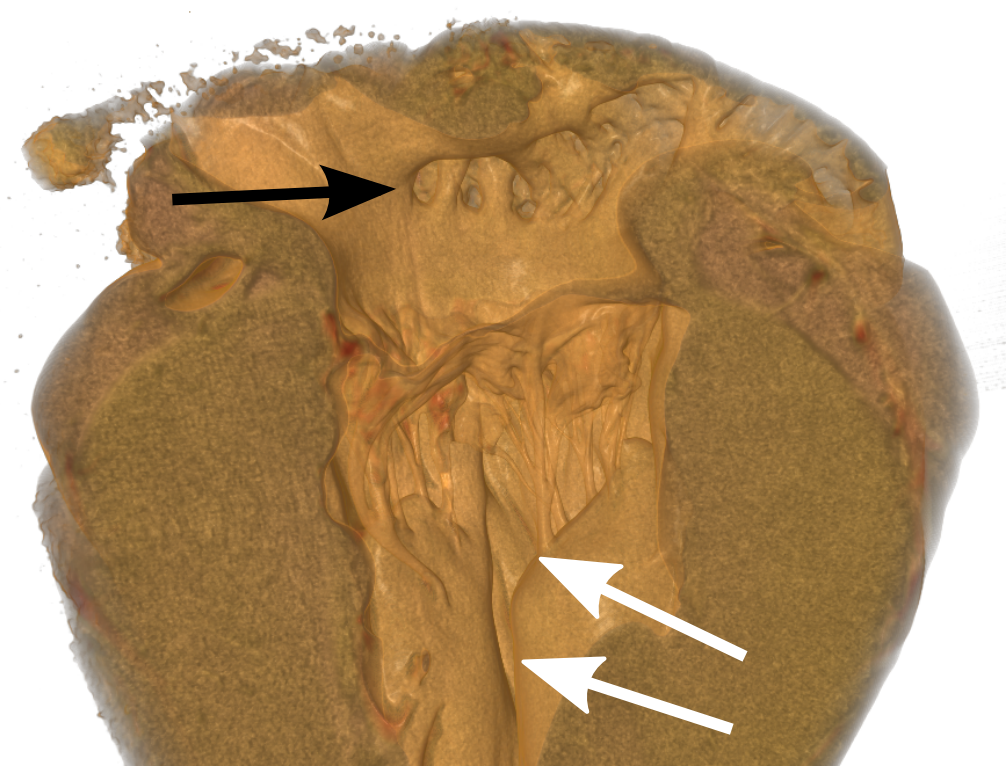

FIGURE 4.3: The structure of the endocardial walls as seen in the left ventricle and the atrium. Note the papillary muscle and trabeculae (white arrows) in the ventricle and the pectinate muscles in the atrium (black arrow).

- Coronary vasculature: Being permanently active, the heart needs a reliable and powerful supply of oxygen and nutrients. Only in small animals where the heart walls are smaller than on the order of $1 \mathrm{~mm}$, direct diffusion from the atria and ventricles into the myocardium is sufficient to play a significant role for the muscular oxygenation.

In larger hearts however, oxygen and nutrient are provided by a system of blood vessels, the coronary vasculature, which branches directly from the aorta. This vascular system is omnipresent in the myocardium, and traverses it whenever necessary, although the larger vessels run mainly on the myocardium or very close to the surface [57]. In two-dimensional cross-sections, the blood vessels can be seen as circular obstacles where the response to external electric fields follows equation (4.6).

For further references on cardiac anatomy, see e.g. [56].

In the second type of heterogeneities the myocardium does not abruptly cease to exist. This type contains modifications of cardiac tissue, by natural causes such as fat deposits, but also scarred tissue after surgeries or fibrotic tissue after myocardial infarctions. These heterogeneities may be locally confined or nearly everywhere in the heart and they may show different degrees of severeness. While it may be impossible to give analytical results of how they react to external fields, they certainly can serve as WEHs, as long as they change the relation between intra- and extracellular space enough to induce current flows across the cell membrane. 
Heterogeneities in the sense of this type do not need to be of different tissue types. Also local changes of the fiber orientation as described in section 2.3.5 can effectively result in conductance changes and thereby lead to wave emissions.

Of all the different kinds of heterogeneities, there is one with distinct properties, it is ubiquitous in the myocardium, there is absolutely no wave propagation inside and its behavior can be described exactly: The coronary blood vessels. For these reasons I will concentrate in this chapter on the interaction of vasculature with electric fields and how it may be used to influence arrhythmias like fibrillation.

\subsubsection{Cardiac Vasculature}

Since blood vessels are one of the main candidates for wave emissions in the bulk tissue, the geometric structure of the coronary vascular tree is of primary interest. Additionally to theoretical considerations, luckily some of its properties like size and connectivity are relatively simple to measure and quantify in animal models.

It has long been known that hierarchical biological systems tend to to follow power law scaling in their sizes due to metabolic optimization [58, 62$]$

In 1926, Murray showed in [58] that basic laws for vascular structures can be derived from the assumption that the metabolic work to keep up the blood flow is minimal. Specifically, the volumetric flow rate $q$ is proportional to the cubic blood vessel diameter, $r^{3}$. Conservation of volume at branching points then lead directly to

$$
r_{\text {parent }}^{3}=\sum_{i} r_{i}^{3} \text { for child branches } i
$$

70 years later, Zhou et al. were able to show that the total lengths and volumes of arterial networks, the diameter of common parental artery segments and the flow should follow power laws in order to minimize the metabolic cost for the organism. Similar relations had been found earlier for the pulmonary [63] and renal system [64].

Consider the radius $r$ of a vascular tree stem (see figure 4.4 for the stem/crown nomenclature as used in [62]) and the flow $q$ through this tree. By denoting the total volume of the tree by $V$ and the total length of all vessels by $L$, the following power law relations and relations between the exponents hold: 


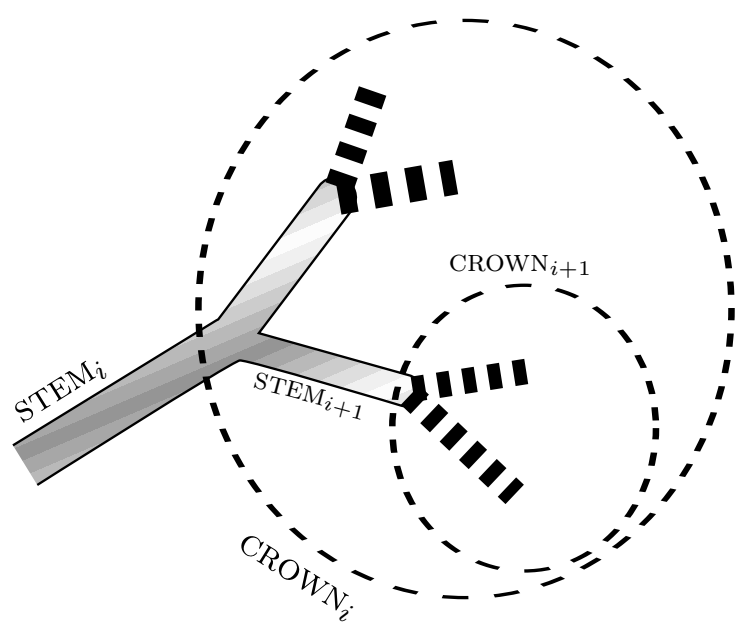

Figure 4.4: Structure of a vascular subtree split into a crown and a stem, as used by 62. Each subtree $i$ starts with a stem $\operatorname{STEM}_{i}$ which continues until the first branching, all following vessels are summed up into the crown $\mathrm{CROWN}_{i}$. This nomenclature is valid recursively for all levels.

$$
\begin{aligned}
V & \propto L^{\xi_{V L}} \\
r & \propto L^{\xi_{r L}} \\
q & \propto r^{\xi_{q r}}
\end{aligned}
$$

with

$$
\begin{aligned}
\xi_{V L}+4 \xi_{r L} & =3 \\
\xi_{r L} \cdot \xi_{q r} & =1
\end{aligned}
$$

With this knowledge it makes sense to expect a power law for the blood vessel size distribution as well. We will define such a distribution in the following way: Consider the vascular tree as a set of segments of length $l_{i}$ (with $L=\sum_{i} l_{i}$ ). The question we now ask is th following: what is the probability $\operatorname{Pr}(r)$ to measure the radius $r$ if you randomly pick one point along the whole tree and does the probability scale as $\operatorname{Pr}(r) \propto r^{\alpha}$ ? And if it does, does $\alpha$ depend on the species or other factors?

\subsubsection{Other Vascular Scaling Laws}

The scaling exponents between e.g. volume and length of vascular trees or flow and vascular size were found to be mostly comparable for pigs, rats, cats, humans and hamsters and for different vascular branches [62]. Figure 4.5 shows the distribution of values for the exponents $\varepsilon^{\prime}$ and $\beta$ from table 2 of Kassab [62] (which can be identified 


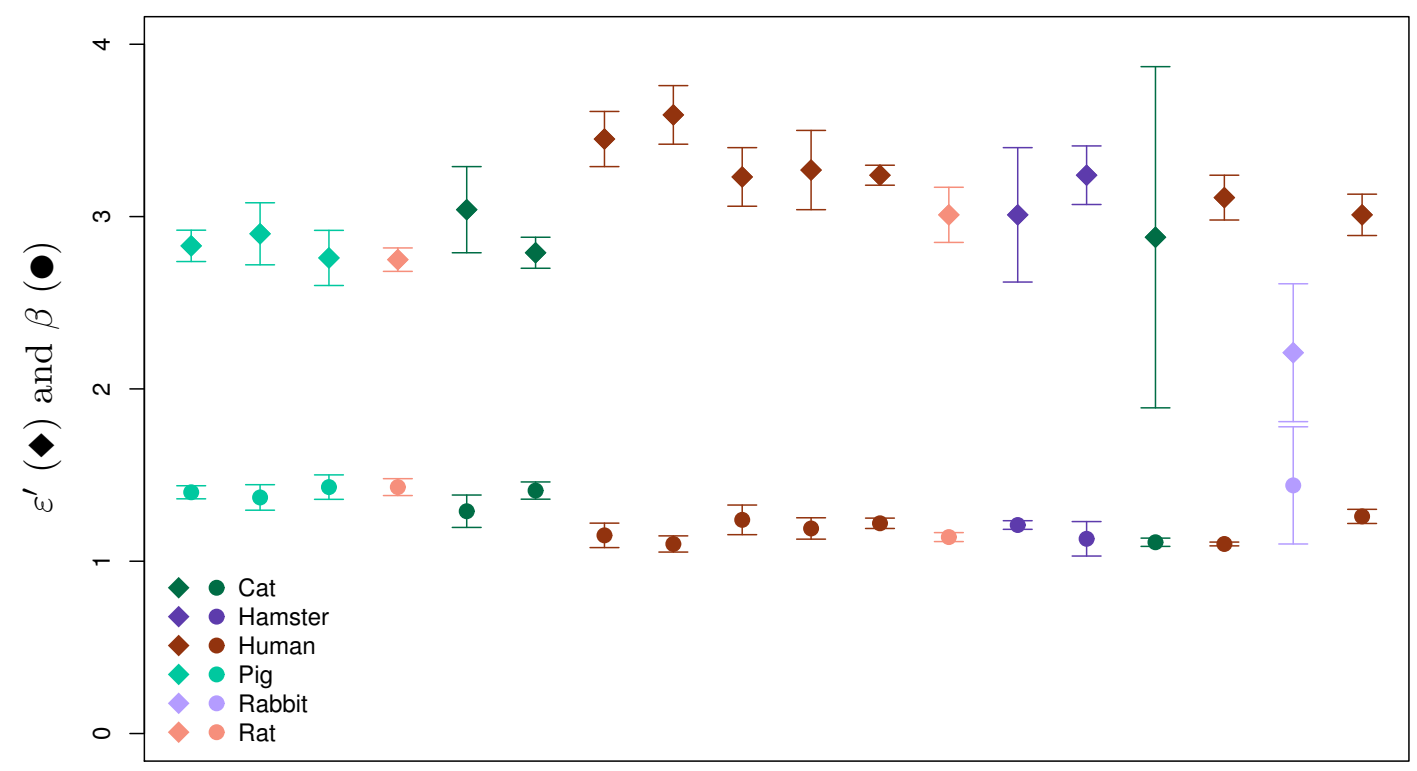

Figure 4.5: Exponents $\varepsilon^{\prime}$ and $\beta$ as given in 62. Each symbol stands for one examination of a vascular branch in several subjects of one species $(4 \leq N \leq 17)$, diamonds

$(\bullet)$ denote measured $\varepsilon^{\prime}$ and circles $(\bullet)$ stand for $\beta$, the error bars show the exponents' standard deviation.

with $\frac{5}{\xi_{V L}}-1$ and $\xi_{V L}$, respectively, from the equations above). Although there are clear differences between some data samples, the exponents are mostly consistent between different species.

\subsubsection{Size Distributions in the Literature}

Although several morphometric relationships as the above-mentioned have been researched before, there are no reports about the overall scaling of cardiac vasculature diameters. Fortunately however, I could use some of the data produced in these previous studies to obtain approximations for the investigated size distribution scaling.

In [65, Kassab et al. examined the diameters and length ratios of arteries before and after branching as a function of the vessel segments order. In their nomenclature, capillaries and arterioles have orders 0 and 1, respectively. Parent segments are of order $n+1$ if their children are of order $n$ and smaller by a specified amount, else they are of order $n$ (the order of their largest child) as well. Kassab et al. measured the smaller vessels (up to order 5-6) in a few samples of histological slices and the larger ones in macroscopic polymer corrosion casts.

For several cardiac tissue types, this article lists, for each vessel segment order $n$, how many segments $\left(N_{n}\right)$ of the respective order were found, their mean diameter $\overline{D_{n}}$ and mean length $\overline{L_{n}}$. Under the assumption that the length $L$ and diameter $D$ of a segment are not correlated except by its order $n$, this data leads to a size distribution $\operatorname{Pr}(r)$ for 


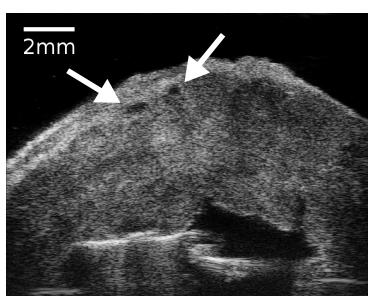

(a) Ultrasound

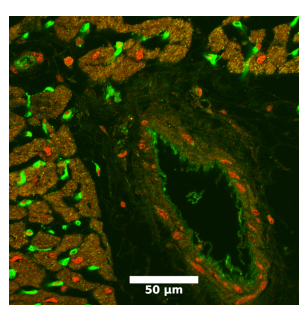

(b) Microscopic

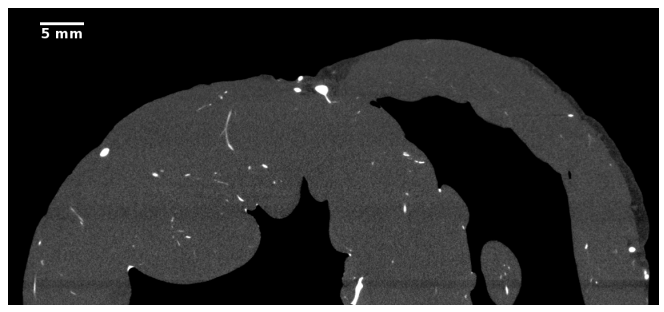

(c) $\mu \mathrm{CT}$

Figure 4.6: Examples of several cardiovascular blood vessel imaging methods. (a) Ultrasound cross section through a rabbit ventricle with two large blood vessels (arrows). Image courtesy of Jan Christoph, Göttingen (b) Laser scanning microscope fluorescence image (microtomic section) of a coronary artery (red: Alpha-actinin, binds to cardiomyocytes, green: Lectin, binds to endothelial cells). (c) $\mu \mathrm{CT}$ cross section through pig left and right ventricle (gray). Blood vessels are visible throughout the tissue, filled with contrast agent (white in this representation).

the given data set at $r_{n}=\overline{D_{n}} / 2$ :

$$
\operatorname{Pr}\left(r_{n}\right) \propto \overline{N_{n}} \overline{L_{n}}
$$

\subsection{Blood Vessel Measuring}

There are several methods to experimentally measure the sizes of coronary blood vessels ex vivo, some of which are also, to a certain extent, applicable in vivo.

\subsubsection{Measurement methods}

For sufficiently large vessels or vessels that are close enough to the surface, the size can be measured with ultrasound imaging. This method is comparably fast and works non-destructively, thus it may be applied during the course of experiments. Figure 4.6 shows examples of ultrasound vascular images and of other methods.

Thie highest spatial resolutions can be achieved with microtomic sections imaged by microscopes, this allows the measurement of blood vessels down to the capillary level. This method comes with several downsides however: Full microtomic imaging of large organs such as the heart requires a lot of manual work since thousands of slices (with a thickness on the order of a few micrometers) need to be cut, scanned and aligned to a three-dimensional image stack. Additionally the process of embedding the tissue into a medium, slicing and staining is prone to distort the organic tissue which makes the subsequent aligning difficult and introduces artifacts in the resulting volume data. 


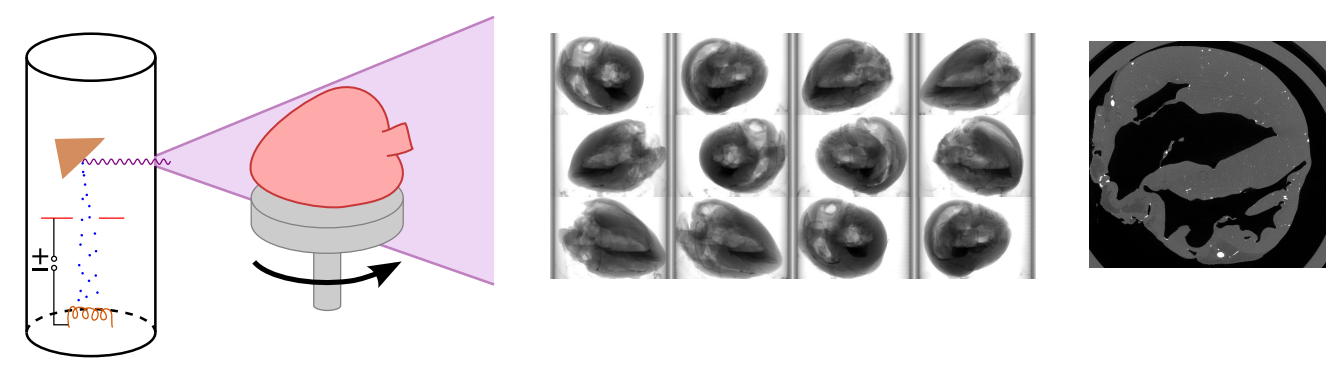

Figure 4.7: In a CT scan, a series of two-dimensional X-ray images is taken of a sample. From this image series the original attenuation volume information can be reconstructed approximatively.

In recent years, other methods have gained increasing popularity: MRI (magnetic resonance imaging) and CT (computed tomography) are not only invaluable for clinical diagnostics but may also be used for research purposes. These imaging techniques produce volume data organized in voxels (volume elements), which can then be analyzed further.

In this work, I will concentrate on $\mathrm{CT}$, which has a generally higher spatial resolution and lower costs. For application in living humans, MRI may be favorable, since it does not have the high X-ray exposure associated with CT scans. For ex vivo imaging, radiation safety is of no immediate concern, since the samples can usually be scanned in fully enclosed scanners which keep radiation at a safe level on the outside.

\subsection{2 $\mu \mathrm{CT}$ scans}

Computed tomography produces volume information of an object by taking a series of two-dimensional X-ray images at different angles (see figure 4.7). In these images the intensity $I$ of each point reflects the attenuation of the X-ray beam through the observed sample according to the Beer-Lambert law

$$
I(u, v)=I_{0} \exp \left(-\int_{0}^{l} \mu(\mathbf{x}(s)) \mathrm{d} s\right)
$$

Here $\mu(\mathbf{x})$ is the attenuation coefficient for a specific volume element of the sample, and the integral runs along the beam from the X-ray source to the point $(u, v)$ on the detector screen.

From the acquired series of two-dimensional images it is possible to calculate the original values of $\mu(\mathbf{x})$ in the sample, by combining filters with a back projection of the attenuation values into the original space. 


\begin{tabular}{ll} 
Medium/Element & Mass Attenuation $\mu / \rho\left(\right.$ in $\left.\frac{\mathrm{cm}^{2}}{\mathrm{~g}}\right)$ \\
\hline air & $1.541 \times 10^{-1}$ \\
\hline water & $1.707 \times 10^{-1}$ \\
blood & $1.695 \times 10^{-1}$ \\
soft tissue & $1.693 \times 10^{-1}$ \\
muscle tissue & $1.693 \times 10^{-1}$ \\
bone & $1.855 \times 10^{-1}$ \\
\hline carbon & $1.514 \times 10^{-1}$ \\
oxygen & $1.551 \times 10^{-1}$ \\
iodine & 1.942 \\
sulfur & $2.020 \times 10^{-1}$ \\
barium & 2.196
\end{tabular}

TABLE 4.1: X-ray mass attenuation coefficients for several media at $100 \mathrm{keV}$ photon energies. Note that these are mass coefficients and thus need to be scaled with the medium density to get the volume attenuation $\mu$ as used in (4.14). Values are cited from $[66,68$

With specialized devices, computed tomography allows spatial resolutions to below $1 \mathrm{\mu m}$, which is three orders of magnitude smaller than in clinical application. Usually the high resolution computed tomography is called "micro-CT" or just " $\mathrm{CCT}$ ".

Since the information returned by $\mu \mathrm{CT}$ is the local attenuation coefficient, measuring blood vessel geometries requires that the blood vessel be linked to X-ray attenuation. Unfortunately, the intensity of water or blood on CT images is practically indistinguishable from the intensity of soft tissue or muscle, as can be seen in table 4.1. The preferred method to make the vasculature visible is the injection of high-attenuation media. (It is also possible to create contrast through replacing fluids by air, for example in the digestive tract. For vasculature this method is not applicable due to the small sizes of the blood vessels.) Elements with high atomic numbers are best suited for this task, but of course also biocompatibility plays a role.

When complete solubility of the applied contrast agent is not required, an aqueous suspension of barium sulfate, $\mathrm{BaSO}_{4}$, can be used. Generally a toxic element, barium can be applied in this form because $\mathrm{BaSO}_{4}$ it is virtually insoluble in water and alcohol ${ }^{1}$. The high attenuation coefficients of barium and sulfur, combined with the increased density of the suspension results in very high attenuation. In this case the attenuation can reach levels where the Beer-Lambert law 4.14 in the presented simple form is not sufficient anymore: because $\mu$ is not independent of the wavelength, typically low energy photons are absorbed more than those with higher energies. This "chromatic" effect know as beam hardening can then lead to artifacts around high contrast areas in the reconstructed image (see figure 4.8 for an example). Another problem connected with the use of $\mathrm{BaSO}_{4}$ as a contrast agent is the sedimentation un undissolved $\mathrm{BaSO}_{4}$

\footnotetext{
${ }^{1}$ approximately $0.0003 \mathrm{~g} / 100 \mathrm{~g} \mathrm{H}_{2} \mathrm{O}$ at $100{ }^{\circ} \mathrm{C}$ 69
} 


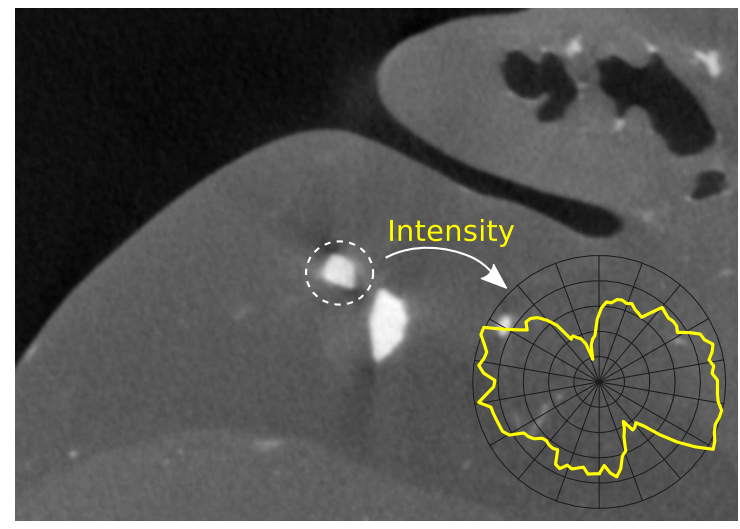

Figure 4.8: Beam hardening in a CT scan: note the dark and bright streaks around the bright spot (vessel filled with contrast agent). The contrast agent attenuates $\mathrm{X}$ rays of some wavelengths more than others, this non-linear behavior can not be compensated by the reconstruction algorithm.

particles which takes place within a few hours, this time span becomes relevant when scanning for an extended amount of time.

Another common contrast agent, attractive because of its high atomic number 53 and because it is relatively harmless to higher organisms, is iodine. In the simplest case, an aqueous solution of $\mathrm{I}_{2}$ and $\mathrm{KI}$ under the name Lugol's solution is used. The disadvantage of this contrast agent for measuring the vasculature is that the dissolved iodine ions are small enough to diffuse easily into the surrounding tissue and increase the X-ray attenuation there as well. This issue can be circumvented by embedding the iodine into larger molecules which diffuse much slower. Iodized alkanes for example are readily available from commercial chemical suppliers.

To minimize artifacts from morphological changes of the surrounding tissue due to drying up or uptake of fluids and to increase the available contrast, it is also possible to fill the vasculature with hardening polymer. Then after the polymer is cured, the surrounding tissue can be corroded away so that only the polymer structure inside the blood vessels remains. This corrosion cast method has been extensively described in the literature, for example in [70] and [71]. After creating the corrosion cast, the resulting structure can be measured either manually or with tomographic methods as described above. The many steps involved make the corrosion cast method is comparatively work intensive, though.

\subsubsection{Preparation and Scanning of Cardiac Tissue}

In the scans in this work, quantitative analysis was done with leaded polymers and 1-iodooctane as contrast agents, some images shown used barium sulfate. 

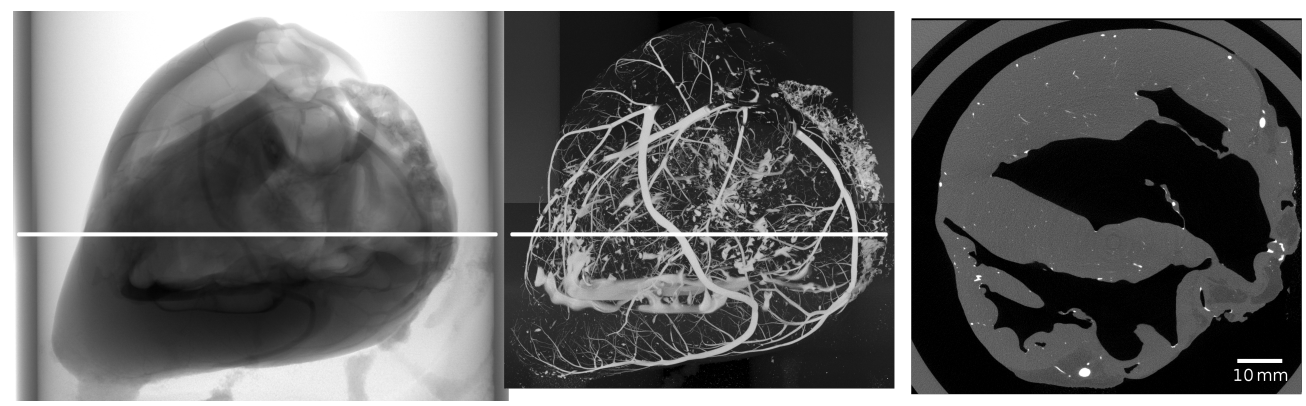

Figure 4.9: Results of $\mu \mathrm{CT}$ scans. The first image shows an unmodified twodimensional view of a pig heart with contrast agent injected into its coronary vascular system (dark tree-like structures). In the second image, three-dimensional volume information was reconstructed from the same specimen. The image shows a maximum intensity projection (MIP), i.e. the intensity of each pixel corresponds to the maximum intensity along its line of sight. The image to the right shows a cross section through the reconstructed volume at the level marked by a white line on the left and middle image. One can easily distinguish air (black, on the outside), tissue (gray) and contrast agent filled vessels (white).

The hearts used in this work originate from animals used in other research experiments. The handling of the animals and the experimental procedure were performed in accordance with the respective national legal requirements. After the end of the experiments, the animals were administered heparin (approximately $400 \mathrm{IU} / \mathrm{kg}$ ) to prevent blood coagulation inside the vascular system after the animal's death.

The heart was retrogradely perfused with the contrast agent at a pressure that was sufficient for a constant flow of medium into the heart. For the dog hearts this was done shortly after the finished experiment while the pig hearts were preserved for a prolonged time in $4 \%$ formalin solution ${ }^{2}$ before administration of the contrast agent.

To achieve the necessary high resolutions, long exposure times (up to several seconds per single image, several images were averaged for noise reduction) and many images (the number of necessary images increases linearly with the resolution, it should be greater than the number of sensor pixels orthogonal to the rotational axis) are necessary. The resolution requirement therefore lead to overall scan times of several hours.

Figure 4.9 shows a $\mu \mathrm{CT}$ scan example, at $60 \mu \mathrm{m}$ resolution. The scan took 4 hours, the resulting volume measures 1400 voxels $\times 1440$ voxels $\times 1377$ voxels, which corresponds to $84 \mathrm{~mm}^{3} \times 86 \mathrm{~mm}^{3} \times 83 \mathrm{~mm}^{3}$. Cardiomyocytes, for comparison, measure about $50 \mu \mathrm{m}$ in length with an approximate $5: 1$ ratio of length vs. diameter.

\footnotetext{
${ }^{2}$ Roti-Histofix, Carl Roth GmbH, Karlsruhe, Germany
} 


\subsection{Automatic Size Reconstruction}

To obtain the size distributions of the coronary vasculature of hearts, scanning and reconstructing the volume information must be followed by size measurements. While the actual measurement of vessel sizes could be done by hand, the time needed for this procedure would render the manual measurement of large numbers of size distributions infeasible.

The problem of obtaining vessel size statistics from a set of voxel data can be split into two separate tasks: First finding where the blood vessels are located and second what their sizes are.

\subsubsection{Locating the Vascular Tree}

The coronary arterial vasculature can be thought of as a forest of disjoint trees of blood vessels in which each tree starts at the aorta. Immediately after the aortic valve, two arteries branch from the aorta to supply the heart muscle itself with oxygen and nutrients [57: The right coronary artery feeds most parts of the right ventricle and the atria, especially the SA node. The left coronary artery branches into two nearly equally sized arteries very shortly after the aorta. Its descendants are the left anterior descending artery, which goes straight down until the apex, and the left circumflex artery, which runs along the heart's base and supplies the left atrium and the remaining parts of the left ventricle.

There exist several methods in the literature to semi-automatically track blood vessels on images based on user assistance [72]. In those cases the user has to manually specify e.g. endpoints of blood vessels and an algorithm finds an optimum way along the blood vessels to connect the given points (figure 4.10). The "optimum way" in this context is usually defined by traversing as few voxels of as much intensity as possible. Although this is already much more efficient than fully manual segmentation, it does not yet make identification of the full vascular tree possible.

Automatic methods to locate all coronary vascular vessel may require the user to enter some initial information such as expected intensity ranges or starting points of the main coronary branches. After obtaining this data however, an automatic algorithm must be able to continue without further user interaction until all blood vessels have been found.

The algorithm I developed in the course of this thesis [73] to find all vessels is based on thresholding and subsequent thinning of the obtained structure, followed by criteria to remove structures caused by scanning artifacts. The algorithm was implemented in 


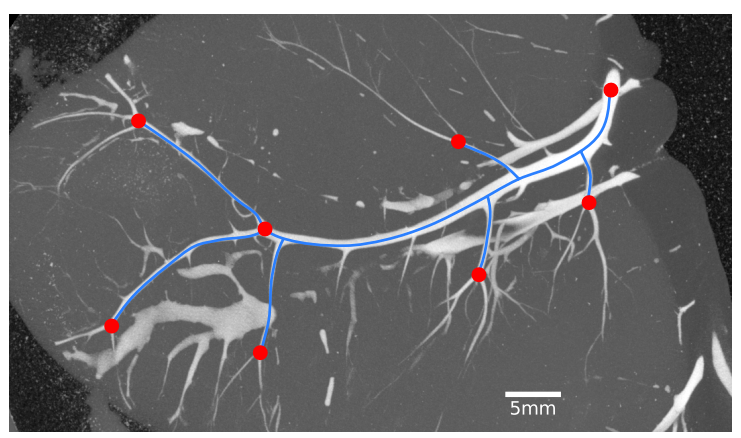

FIGURE 4.10: In the semi-automated vessel segmentation proposed in [72], the user has to manually identify a number of characteristic points of the arterial vasculature on images of the patient. The algorithm then connects these points using the contrast agent as an indicator of the optimum path.

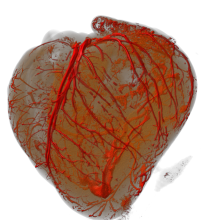

Raw image data

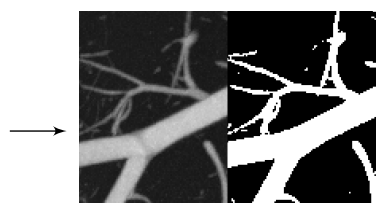

Thresholding

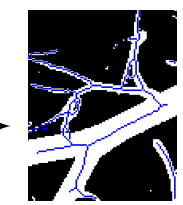

Skeletonization

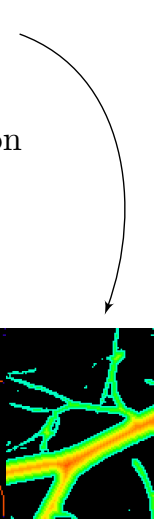

Skeleton cleaning

Size statistic

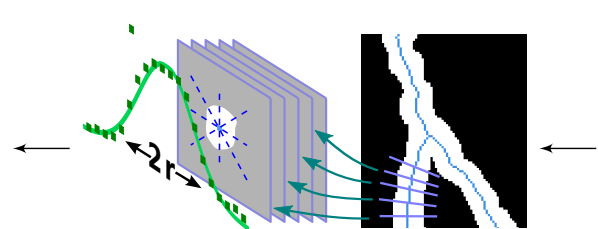

Slice orientation and fit

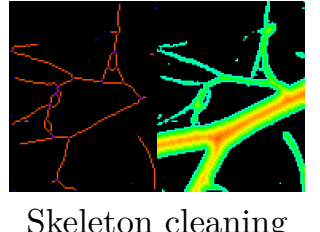

Figure 4.11:

Overview over the general structure of the size measurement algorithm:

- Thresholding

- Skeletonization

- Skeleton analyzation \& removal of surfaces, exceedingly large vessels and small trees

- Orientation and size for each center line voxel

This algorithm creates a list of vessel elements with position and radius from a volume image, the result is plotted graphically as a three-dimensional rendering where each calculated size corresponds to the color at its respective coordinates.

Java as a plugin to the image analysis platform Fiji [74], a distribution of the widely used ImageJ [75] program. In the plugin I made use of numerical libraries provided by the Apache Commons project [76]. An overview over how the program works is shown in figure 4.11

In the first step, the volume image is segmented into high and low intensity voxels according to user input, high intensities correspond to high X-ray attenuation and thus 


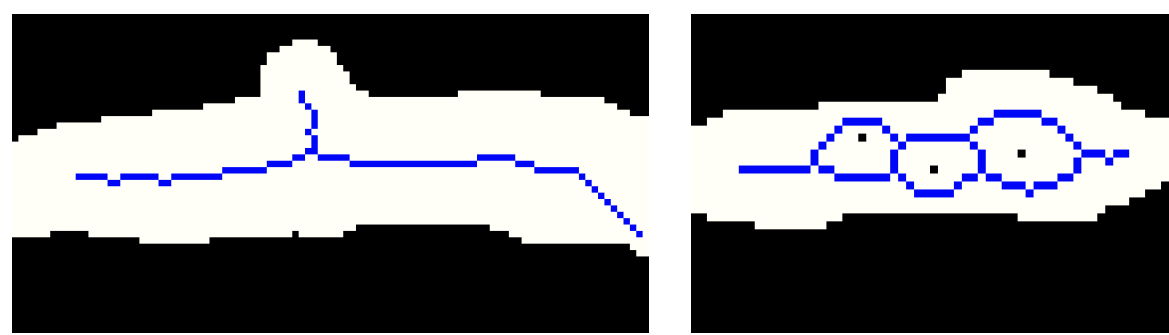

FIgURE 4.12: Visualization of the skeletonization process. Voronoi cell artifacts can be caused by stray black voxels and vessel protrusions may lead to unintended skeleton branches. Note that these sketches are two-dimensional, but the underlying effects occur analogously in three dimensions as well. (First published in [73.)

probably to contrast agent, in the vessels and elsewhere. Thus it is reasonable to assume that the blood vessels' center lines are completely within the set of high-intensity voxels - except those places the contrast agent failed to reach and very small vessels, for which the scanner resolution was not sufficient. To extract a center line of exactly one voxel thickness, the resulting thresholded image was successively thinned using a skeletonization algorithm described by [77]. This algorithm results in a set $S$ of skeleton voxels $\vec{x}$ which contain information about their distance $d$ to the next low intensity voxel: $S=\left\{\left(\vec{x}_{i}, d_{i}\right)\right\}$

The size measurement plugin for this work uses an implementation of the thinning algorithm that existed already as a skeletonization plugin to ImageJ [78, 79].

Without noise and artifacts of preparation and scan in the volume data, this procedure would result in skeleton trees ${ }^{3}$ of voxels that lie in the center line of the coronary vascular system. Unfortunately thinning algorithms like the aforementioned one are sensitive to small voxel classification errors, e.g. due to noise or reconstruction artifacts:

Noise The thinning process is sensitive to single voxels that are wrongly marked as black instead of white, as can be seen in figure 4.12. Low intensity ("black") regions inside volumes classified as high intensity will expand during the thinning process, leaving a thin wall around them (right hand side image in figure 4.12 instead of the expected one-dimensional filament. For several single black voxels, the resulting wall represent the boundaries of the Voronoi cells corresponding to the defects.

Conversely, single white voxels would not be changed and become their own 1 voxel tree. Erroneously marked black or white voxels may arise from noise in the original $\mathrm{X}$-ray images, which can be countered to a certain degree by averaging over many images. This further increases the scan time, of course.

\footnotetext{
${ }^{3}$ Here trees are understood in the sense of graph theory: A tree is a connected graph where the edges do not form loops.
} 
Skeletonization ambiguities Another problem may arise when the vessel shows protrusions which can lead to spurious branches of the skeletal tree (left image in figure 4.12. These unphysiological branches need to be removed in later stages.

Spilled contrast agent While filling the coronary vascular system with contrast agent, sometimes spilling of contrast agent on the outside of the sample cannot be avoided completely. This leads to additional high intensity regions in the resulting volume data which produce yet more artificial skeleton trees.

Contrast agent in the ventricles and atria Perfusion of whole hearts with contrast agents is usually done retrogradely through the aorta. At a sufficiently high pressure, the contrast agent will flow into the coronary arteries which start shortly after the aortic valve. Some contrast agent however inevitably also passes through the aortic valve into the left ventricle and possibly also into the left atrium. Contrast agent returning through the venous system may also end up inside the right atrium.

In the reconstructed CT image, the ventricle and/or atrium can then be clearly distinguished from the surrounding tissue. Depending on the miscibility of the contrast agent with the previous filling fluid and on the relative density, there will be one or more relatively large connected regions of high intensity, at the bottom or top of the left chambers and in the aortic trunk which are also reduced to some kind of skeletal structure in the skeletonization process.

To yield a skeleton of one single voxel thickness as a faithful representation of the vascular structure, the above mentioned artifacts are removed in a number of additional steps:

- Remove voxels whose distance $d_{i}$ is larger than a predefined threshold $d_{\max }$. This threshold is usually chosen to be slightly larger than the diameter of the largest coronary artery where it joins the aorta. After this step, the skeleton is reduced to $S=\left\{\left(\vec{x}_{i}, d_{i}\right) \mid d_{i} \leq d_{\max } \forall i\right\}$.

This pruning removes unwanted contributions from the aorta or even contrast agent filled ventricles or atria.

- Additionally all skeleton points with 6 neighbors (out of the possible 26 neighboring lattice positions) or more are removed. This procedure cleans the skeleton from surfaces which stem from dark voxels included within white areas.

- In a next step, the remaining skeleton is segmented into a number of disjoint trees using the AnalyzeSkeleton [79] Fiji plugin. If there are loops within the skeleton graph, the edge with the smallest size $d$ is removed. There are two criteria in place 
which need to be fulfilled by skeleton trees and the branches of each tree, otherwise they are removed:

1. For each tree $i$, count the number of voxels $n_{i}$ in this component $i$. Remove the tree if $n_{i}<c \sum_{i} n_{i}$ with $c$ being a fixed fraction parameter, typically 0.05. This is an effective measure to remove artifacts from small spots of contrast agent in other places than the blood vessel system, e.g. on the atrial or ventricular endocardium.

2. For each leaf branch $j$ (that has only one voxel with more than two neighbors) get the length $l_{j}$ and distance to black $d_{j}$ at the connection to the main tree. Remove this leaf branch if $l_{j}<d_{i}$. This removes spurious branches as in figure 4.12 , image on the left.

From the thus reconstructed vasculature skeleton, it is possible to accurately measure the blood vessel sizes.

\subsubsection{Accurate Size Measurements on the Vascular Tree}

The size of a vessel is measured in the two-dimensional plane perpendicular to the vessel direction. In the case of contrast agent filled vessels, this problem reduces to finding the size of a shape which best describes the intensity distribution as shown in the examples of figure 4.13 .

To derive the cross section for each point $i$ on the skeleton structure, the reconstruction program estimates the local direction $t_{i}$ from the positions of the neighboring skeleton voxels. Up to $4 \cdot d_{i}$ neighboring voxels are considered for this direction approximation, where $d_{i}$ is the same distance as used for the branch pruning above.

There exist a number of algorithms (see the info box on the facing page) to measure the size of a roughly circular shape in 2D. However, most of these algorithms are computationally expensive because they operate on the full two-dimensional plane. This means that the runtime cost $T\left(r_{i}\right)$ to measure the radius $r_{i}$, including actually acquiring the plane data from the raw volume, grows at least quadratically with the radius: $T\left(r_{i}\right) \in \Omega\left(r_{i}^{2}\right)$. One additional problem is that the user (or program) usually needs to provide at least the approximate expected size in advance in order to deliver a fitting cross-sectional plane.

In contrast to this, the algorithm used in this work does not take into account the whole plane, but only samples the volume along a number of directions, which enables us to bound the computational complexity linearly from above: $T\left(r_{i}\right) \in \mathcal{O}\left(r_{i}\right)$. 


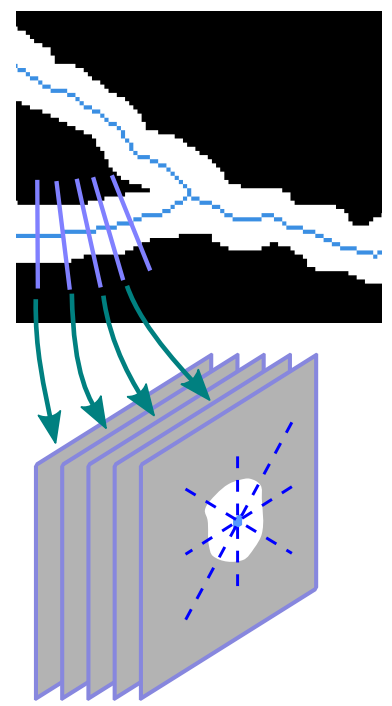

(a)
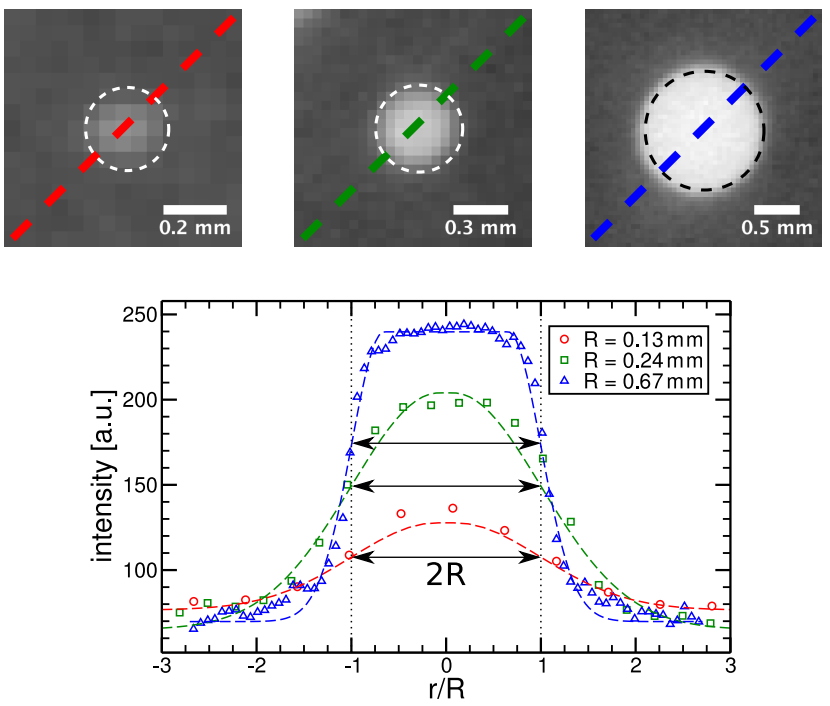

(b)

Figure 4.13: Vascular sizes are measured from cross sections through the CT image. (a) Schematic cross sections of a blood vessel along its center line. (b) Typical blood vessel cross sections, the diameter may span the whole range from several millimeters down to the resolution of the acquired image, $60 \mu \mathrm{m}$ in this case. Brighter pixels correspond to higher X-ray attenuations. Figure first published in [45].

\section{Detection and measurement of circular objects}

Detecting circles and ellipses is a common problem in digital image analysis, with many possible solutions:

Area detection Once the included area $A$ has been calculated, e.g. with a threshold operation, calculate the center as the center of mass and the radius as $r=\sqrt{A / \pi}$.

Hough transform Requires an edge detection of the image first. All pixels on the boundary of the circle add weight to all points in the parameter space $\langle x, y, r\rangle$ which are compatible [80]. The maximum in this parameter space then constitutes the measurement. The parameter space grows to 5 dimensions for general ellipses.

Boundary fit The boundary, once detected, is fit to a parametric curve describing the circle or ellipse.

All these methods share the limitation that they need to process $\mathcal{O}\left(r^{2}\right)$ pixels.

Specifically, the size estimation program repeats the following procedure for each skeleton voxel $i$ until convergence is reached or a maximum number of iterations $n_{\max }$ has passed:

- Sample intensity data along 4 lines through the estimated vessel center, perpendicularly to the vessel direction, angled at $45^{\circ}$ to each other. The length of these lines is chosen to be three times larger than the previously estimated diameter $2 r_{i}^{n-1}$. During the first run $(n=0)$, use the skeleton coordinates as the center and the distance to the background $d_{i}=2 r_{i}^{0}$ as initial guess for the diameter. 


\section{The Gaussian step function}

In the "Gaussian step function" 4.15 the parameter $\sigma$ denotes the width of the fall-off region, a measure for the resolution of the tomography data. For $\sigma=0$, it becomes the ideal step function, while for $\hat{r}=0$ we get the well-known Gaussian function $I(x)=$ $b+a \cdot e^{-\frac{x^{2}}{2 \sigma^{2}}}$

For a typical curve of the Gaussian step function, see the schematic below or figure 4.13b.

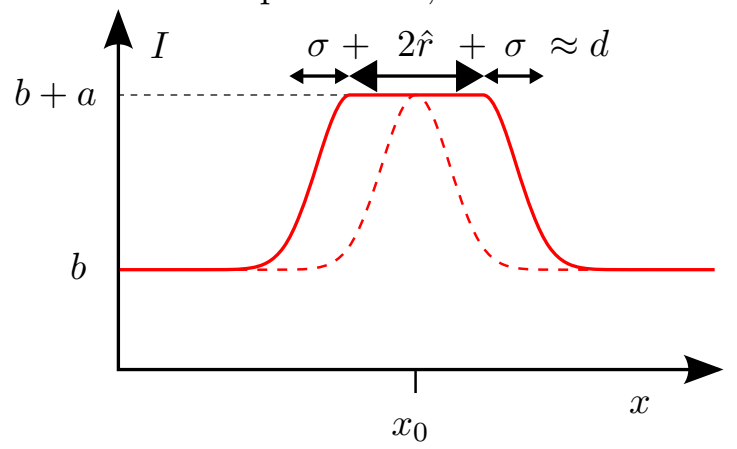

- Fit the following function describing a double step function with a Gaussian fall-off to the intensity values $I(x)$ along each line:

$$
I(x)=I(x)_{a, b, \hat{r}, \sigma, x_{0}}=\left\{\begin{aligned}
b+a & \text { if }|x|<\hat{r} \\
b+a \cdot \exp \left(-\frac{\left(\hat{r}-\left(x-x_{0}\right)\right)^{2}}{2 \sigma^{2}}\right) & \text { if }|x|>=\hat{r}
\end{aligned}\right.
$$

Here $b$ is the base intensity of the background and $b+a$ the intensity of the blood vessel. The true radius of the blood vessel can now be estimated by $r_{i}^{n}=\hat{r}+\sigma$, $x_{0}$ gives the center of the vessel.

- In order to accept these values, $2 r_{i}^{n}$ must be between $40 \%$ and $70 \%$ the length of the sampling line.

Otherwise, the procedure is repeated using larger or smaller values, respectively, for the size of the blood vessel, with the vessel center taken from the latest estimates.

This algorithm finally yields a vessel size for every point along the skeleton, along with a more accurate spatial location for each point. Figure 4.14 shows a graphical representation of such a reconstruction, the vascular sizes are color-coded and overlayed over a maximum intensity projection of the original CT scan.

\subsubsection{Accuracy tests}

To evaluate the accuracy of the presented method and the effective resolution of the $\mu \mathrm{CT}$ scanner, I tested the procedure for cylindrical objects of known sizes. 


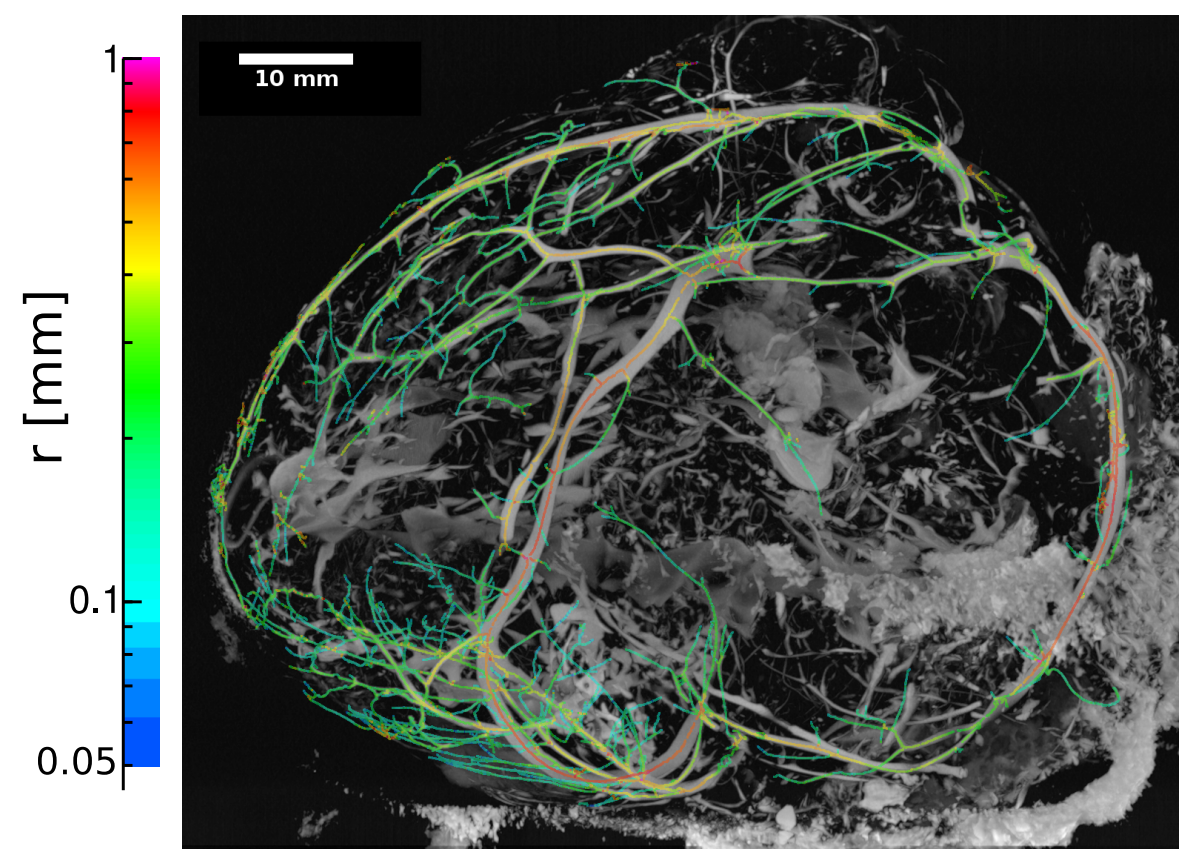

FIgURE 4.14: Graphical result of a vascular size reconstruction. In this maximum intensity projection, the color-coded radii are overlaid over the original image.

I used glass fibers of known diameters for this purpose, glued together in a tree-like structure. I scanned this phantom with the $\mu \mathrm{CT}$ scanner which was also used for the size analysis of the porcine hearts.

The resulting detected radii are shown in figure 4.15, they agree with the glass fibers' nominal sizes with an error of less than 1 voxel length.

\subsection{Measuring the Size Distributions}

To measure the size distribution in the cardiac coronary vasculature, we acquired $\mu \mathrm{CT}$ data from the atria and ventricles of adult beagle dogs, which were used in in vivo and ex vivo LEAP experiments previously (for more details on these experiments and the $\mu \mathrm{CT}$ reconstruction, see [45]). This also gave us the opportunity to retrospectively relate potential peculiarities in the dynamical behavior during the experiment to the structure of the heart.

\subsubsection{Data Acquisition of Dog Heart Vessel Sizes}

Five atrial and three ventricular dog heart wedges were scanned [45] in a $\mu \mathrm{CT}$ scanner ${ }^{4}$ at $25 \mathrm{\mu m}$ voxel resolution in all directions, after injection of $1-2 \mathrm{~mL}$ of Microfil ${ }^{5}$ immediately

\footnotetext{
${ }^{4}$ GE CT120, GE Healthcare, Little Chalfont, UK

${ }^{5}$ Flow Tech Inc., Carver, MA
} 


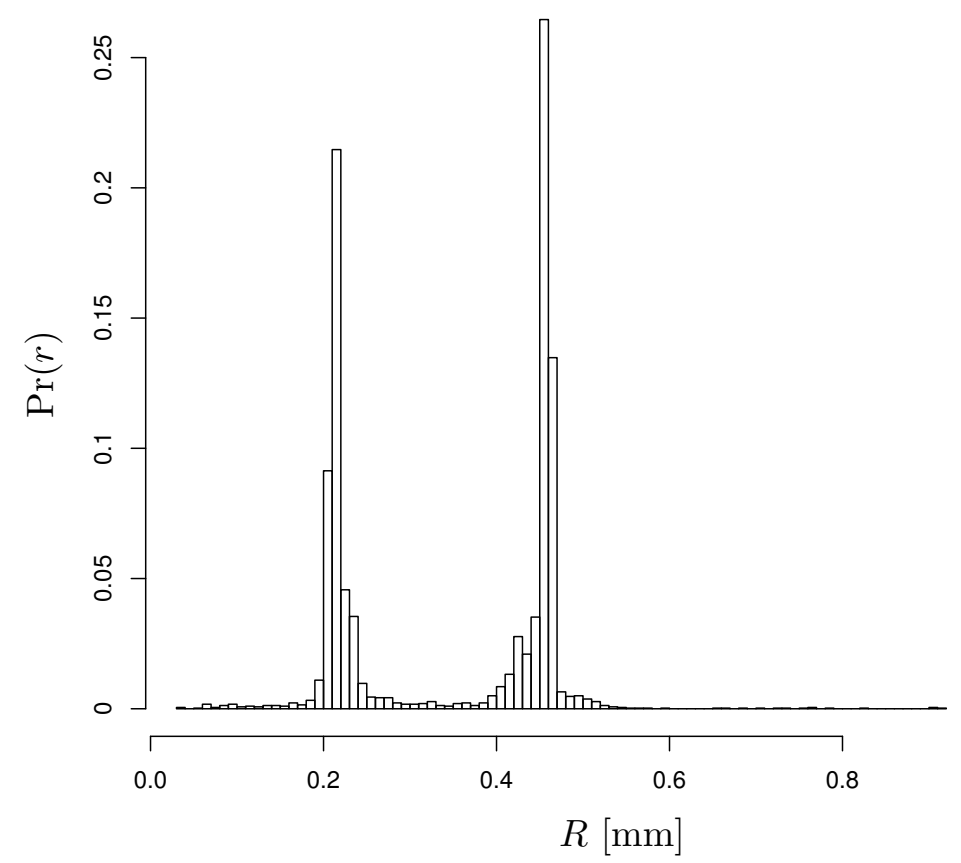

FiguRE 4.15: The detected "blood vessel" sizes for a phantom made of glass fibers. The voxel resolution was $60 \mu \mathrm{m}$, the glass fibers had nominal radii of $0.25 \mathrm{~mm}$ and $0.50 \mathrm{~mm}$.

following the ex vivo experiments, via the same catheter that was used for the perfusion during these experiments. This contrast agent's radiopacity is based on its lead content; it is fluid initially, but polymerizes in the hours following administration.

The scanner's X-ray source was set to $80 \mathrm{kV}$ at $32 \mu \mathrm{A}$. From 1200 equally spaced projections over $360^{\circ}$ we obtained reconstructed images of 25 and $50 \mu \mathrm{m}$ voxel resolution.

The volume images were cropped and preprocessed in order to lessen the influence of the catheter which was clearly visible and to reduce the memory requirements. To decrease the random noise of the data, a spatial median filter (over a $3 \times 3 \times 3$ voxels cube) was applied as necessary.

I applied the size reconstruction algorithm described in 4.4 to these data sets on high performance computers available at the Max Planck Institute for Dynamics and SelfOrganization (16 CPU cores, Intel Xeon E7340 at $2.4 \mathrm{MHz}$ with $4 \mathrm{Mi}$ B cache, $64 \mathrm{Gi} \mathrm{B}$ RAM, Linux 2.6.32). The reconstruction procedure took between one and two hours for each data set on these computers.

\subsubsection{Porcine Blood Vessel Sizes}

To compare the blood vessel size distribution to that in hearts of another species, I adapted the procedure that was used previously in dog hearts and performed supplementary size statistic measurements in pig hearts. 
I prepared $N=7$ pig hearts for $\mu \mathrm{CT}$ vasculature scans after they were used in the LEAP experiments described in more detail in the following chapters 6 and 7 .

After the end of the experiment, the hearts were first perfused with a hyperkalemic solution to assure a diastolic state and then flushed and covered with $4 \%$ formaldehyde (Roti-Histofix $4 \%^{6}$ ) in order to preserve the heart until the scan.

As contrast agent I chose a mixture of $10 \%$ iodo-octane $\left(\mathrm{C}_{8} \mathrm{IH}_{17}{ }^{7}\right)$ and $90 \%$ dodecane $\left(\mathrm{C}_{12} \mathrm{H}_{26}{ }^{7}\right)$. The iodine content gives enough X-ray contrast to show even small vessels, combined with several advantages over the previously used $\mathrm{BaSO}_{4}$. Since it is a fluid, it will not block the capillaries and there is no risk of sedimentation as could be observed with $\mathrm{BaSO}_{4}$ suspensions during prolonged scans. Additionally we observed several cases of $\mathrm{BaSO}_{4}$ diffusion into the tissue (possibly at fissions of the aortic walls), an effect that should be much less pronounced due to the higher molecular weight of iodo-octane. It shares these properties with the, commercially not available, oil-based contrast agent Angiofil [81.

At least one hour before the $\mu \mathrm{CT}$ scan, the heart was removed from the formaldehyde solution and the formaldehyde in the heart chambers and on the surface was removed. The coronary vasculature was then manually filled with the contrast agent via catheters leading directly into the left and right coronary arterial branch, respectively. We found that staining the contrast agent with red Sudan III $^{8}$ helped to apply the correct volume and pressure while filling the vascular system.

The pig hearts were placed in a custom-made acrylic glass container onto low-density foam plastic which was of significantly lower intensity than the water-based heart tissue. The acrylic glass container also doubled as a "water" phantom of $0 \mathrm{HU}^{9}$ intensity. In this container, the pig hearts were scanned in a Phoenix Nanotom $\mathrm{\mu CT}$ scanner (GE), at $100 \mathrm{kV}$ and approximately $90 \mu \mathrm{A}$ with 2600 angular steps. Scanning of one sample took approximately nine hours. The scanner images were automatically corrected pixel-wise by their respective dark-image and response to increasing X-ray intensity.

After the scan, the images were corrected for potential movement artifacts during the scan and the volume image was reconstructed at a spatial resolution of around $60 \mathrm{\mu m}$. The contrast agent in the blood vessels had an intensity of up to approximately $1500 \mathrm{HU}$ (see also figure 4.16 for a visualization of the intensity values and figure 4.17 for a threedimensional rendering).

\footnotetext{
${ }^{6}$ Carl Roth, Karlsruhe, Germany

${ }^{7}$ Sigma-Aldrich, Seelze, Germany

${ }^{8}$ Sigma-Aldrich, Seelze, Germany

${ }^{9}$ Hounsfield Units (HU) denote the relative X-ray attenuation of a medium. The Hounsfield scale is normalized so that vacuum has $-1000 \mathrm{HU}$ and water has $0 \mathrm{HU}$.
} 

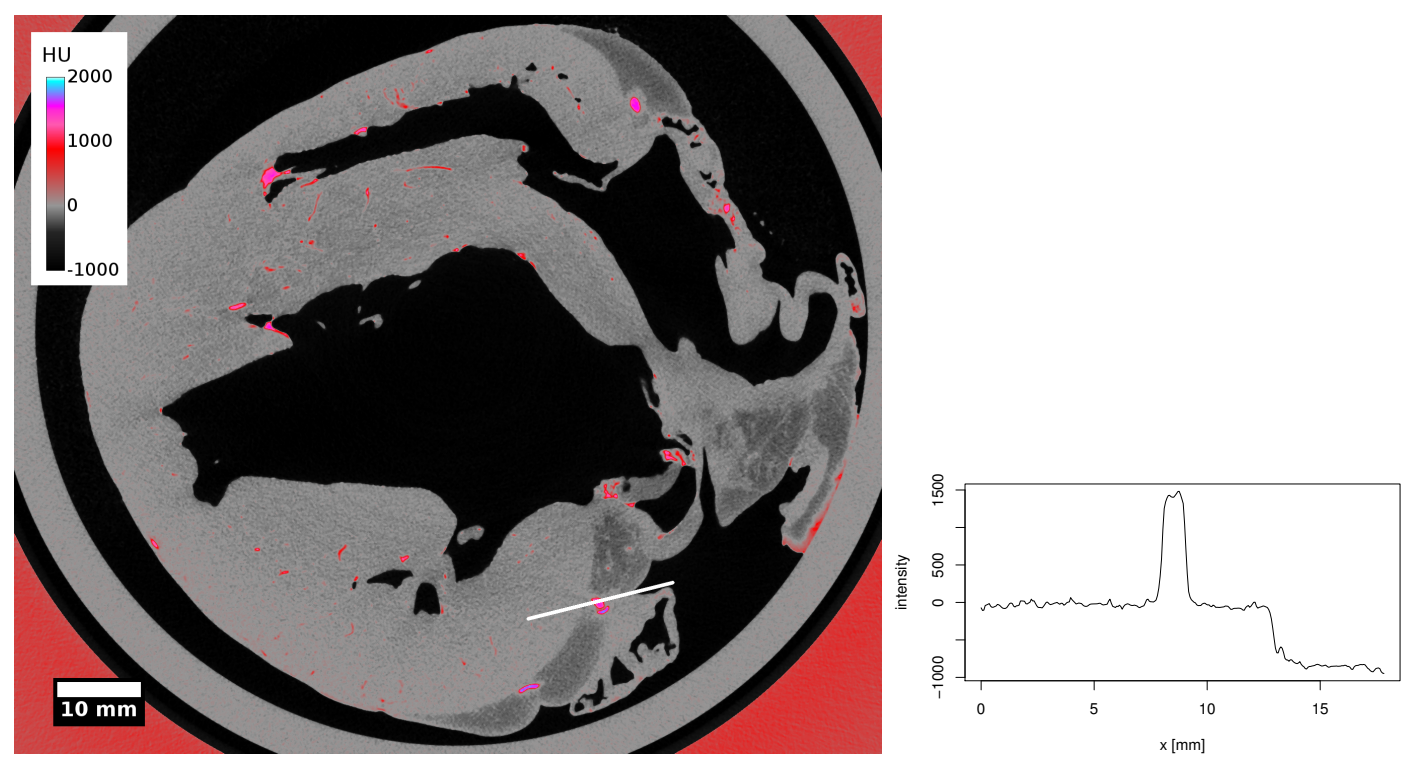

Figure 4.16: Cross section through the reconstructed $\mu \mathrm{CT}$ volume of a pig heart. The intensity of muscle tissue is at around $0 \mathrm{HU}$, fatty tissue is slightly below. Blood vessels that are filled with contrast agent are at about $1000 \mathrm{HU}$ and above, a value that is comparable to bones. The left ventricle (LV) is at the bottom, the RV at the top, also visible are parts of the atrioventricular valves and of the atria (in the right image half). The intensity values along the white line in the lower right are shown in the plot on the right hand side.

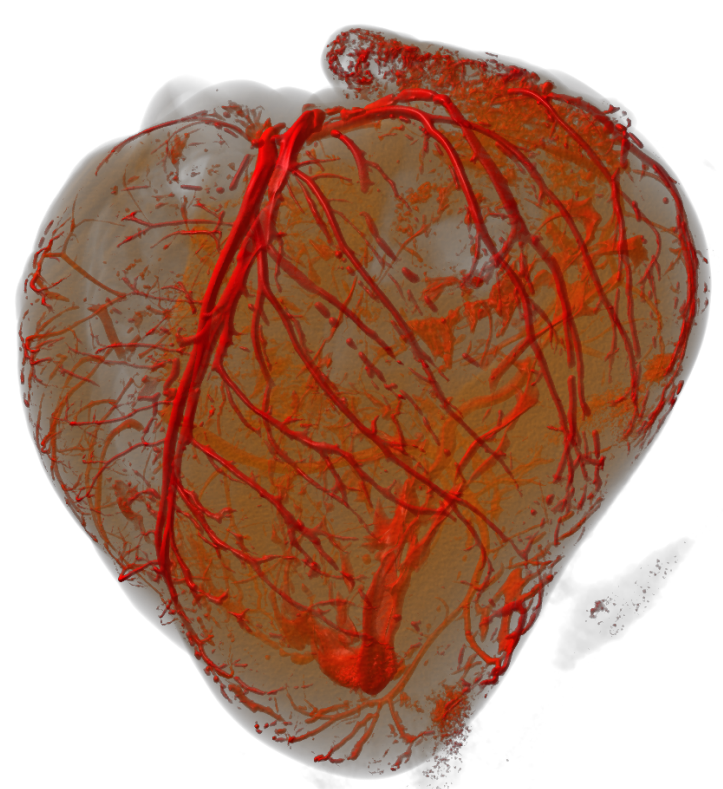

Figure 4.17: The reconstructed view of a pig heart. Tissue is shown as semitransparent, the contrast agent is red. The LV is at the right, the RV at the left and the apex at the bottom of the figure. The spatial resolution for the rendered image is $150 \mu \mathrm{m}$, the heart is $83 \mathrm{~mm}$ wide. 
After preprocessing as described for the dog hearts (mostly cutting away unnecessary parts of the volume), the volumes of approximately $1500^{3}$ voxels were subjected to the same size analysis programs on the Max Planck Institute's computing cluster. This computation took approximately 9 hours for each data set.

\subsection{Electrical Tissue Activation}

As stated before, heterogeneities may serve as excitation wave sources (wave emitting heterogeneities, WEH) under externally applied electric fields. In quiescent tissue, these waves will eventually excite the whole tissue, this process can be described by the activated area $A(t)$, the part of the tissue which has been activated already. Observing $A(t)$ under controlled conditions allows to draw conclusions about the underlying structure of the tissue and its interaction with electric fields. The methods described in this section have been published in [45] and more details are given in [82].

$A(t)$ will grow as the cumulative volume of the emitted waves, initially proportionally to $t^{D}$ for $D$ dimensions. The time $\tau$ required to activate all of the tissue can also be quantified, at least if the $N$ WEHs are distributed homogeneously in the medium of volume $V$. In this case, the distance $d$ between two neighboring WEHs and the wave propagation velocity $v$ lead to

$$
\tau(\mathbf{E})=\frac{d}{v}=\frac{1}{v}\left(\frac{V}{N(|\mathbf{E}|)}\right)^{\frac{1}{D}} .
$$

Since the minimum size for obstacles to turn into WEHs decreases monotonically with growing $E$, more and more obstacles are recruited at higher field strengths, leading to shorter activation times. The minimum size $R_{\text {min }}$ of an obstacle to achieve a sufficient depolarization $\phi_{0}$ for a given electric field strength $E$ is plotted in figure 4.18. For sufficiently small obstacle sizes, $R_{\min }$ decays like $\frac{1}{E}$.

\subsubsection{Activation Time Measurement}

Several methods exist to measure the tissue activation: Electrodes sampling the membrane potential at different locations [83, mapping of mechanical contraction via ultrasound [84] and the use of voltage or ion concentration sensitive dyes all allow to obtain data on how excitation fronts propagate and which effects heterogeneities have on the tissue. Because it provides the best spatial resolution and is less invasive than the other methods, we focused on the latter option to measure the activation times $\tau$ of cardiac tissue. 


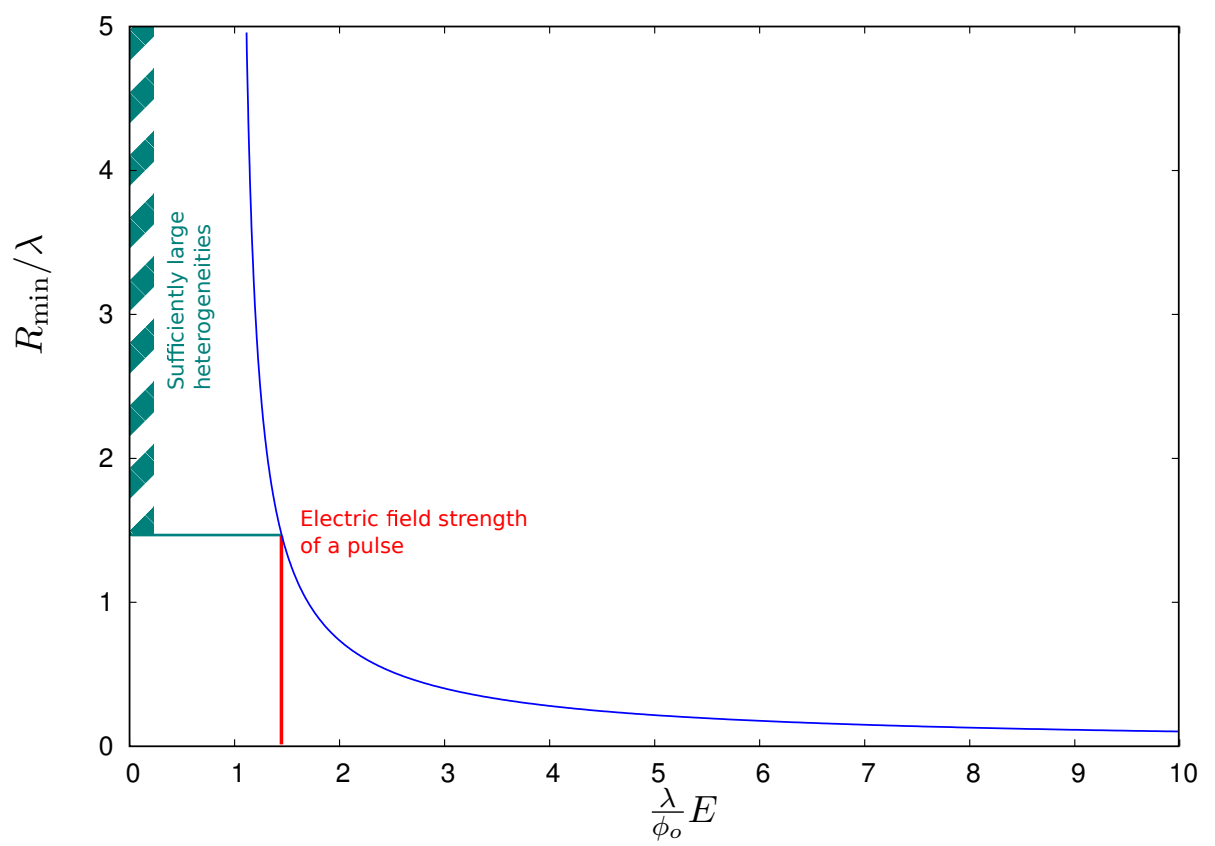

FIgURE 4.18: The rescaled minimum radius $R_{\min } / \lambda$ of a circular obstacle which is sufficient to depolarize the tissue at its boundary by $\phi_{0}$ when an electric field $E$ is applied. For the elecric field denoted by the red line, only obstacles with the sizes marked in green will be sufficiently depolarized to emit a secondary wave.

\subsubsection{Optical Mapping of Cardiac Electrical Activity}

Optical mapping is an experimental technique developed to observe the change of (mostly cellular) states in tissue through the use of dyes which change their optical characteristics depending on the properties to be examined. Typically used are fluorescent dyes whose emission wavelengths are sensitive to either ion concentrations (such as fluo-4 [85, 86], which reacts on the intracellular $\mathrm{Ca}^{2+}$ concentration) or the membrane potential (for example $d i$-4-ANEPPS [87, 88]).

In our experiments, we used the membrane potential sensitive dye di-4-ANEPPS, which attaches preferentially to lipid bilayer membranes, to measure activation times and to monitor the tissue's electrical activity during sinus rhythm, fibrillation and defibrillation attempts. di-4-ANEPPS is excited by blue and green light around $470 \mathrm{~nm}$ and emits around $630 \mathrm{~nm}$ [88, 89]. In response to depolarization of the cell, the fluorescence is decreased and the emission spectrum is slightly red-shifted, which allows to reconstruct the activation to be reconstructed from optical recordings. 


\subsubsection{The Experimental Setup of Optical Cardiac Activation Time Mea- surements}

Optical mapping for activation maps was performed by Philip Bittihn, Flavio Fenton, Stefan Luther and Amgad Squires [45]. Atrial $(n=5)$ and ventricular $(n=7)$ wedge preparations of beagle dog hearts were cannulated through the coronary arteries and perfused with normal Tyrode solution (at 50-80 $\mathrm{mmHg}, 37.0(5)^{\circ} \mathrm{C}$ ) bubbled with carbogen $\left(5 \% \mathrm{CO}_{2}, 95 \% \mathrm{O}_{2}\right)$. The tissue was immobilized by administration of blebbistatin, a myosin II inhibiting excitation-contraction decoupler (approximately $7 \mu \mathrm{mol}$ ) and stained with a bolus of di-4-ANEPPS at a concentration of $10 \frac{\mu \mathrm{mol}}{\mathrm{L}}$.

The optical setup was illuminated by LEDs (LUXEON, maximum power $5 \mathrm{~W}$, peak wavelength $530 \mathrm{~nm}$ ). The tissue was placed horizontally into a glass dish which allowed imaging from above and below (endo- and epicardium) simultaneously.

High-speed EMCCD cameras (CASCADE $128+$ with $128 \times 128$ pixels, 16 bit, Photometrics, USA) fitted with low pass filters at $610 \mathrm{~nm}$ were used to record the electrical tissue activity, controlling and recording of the signals was done with a custom-written pro$\operatorname{gram}^{10}$.

To measure the tissue activation times' dependence on the strength of applied electric fields, two stainless steel mesh electrodes (approximately $2 \times 4 \mathrm{~cm}$ ) were placed into the Tyrode bath at opposite sides of the tissue, at a distance of about $12 \mathrm{~cm}$. A custom program written in LabView (National Instruments, USA) produced pulses which were amplified by a high-power amplifier (BOP 100-4M, up to $\pm 100 \mathrm{~V}$ and $\pm 4 \mathrm{~A}$, Kepco, USA) and delivered to the electrodes. For initially quiescent tissue, series of such pulses with increasing field strengths allowed to measure the tissue's response.

We described this setup and the results of the experiments in Luther et al. [45], where additional details, mostly pertaining to LEAP efficiency experiments, are given.

\subsubsection{Optical Mapping Image Analysis}

In optical mapping with voltage sensitive dyes, the change in amplitude in the optical signal due to the depolarization of the cardiac tissue is much smaller (typically a few percents) than the naturally occuring spatial variance (can be several orders of magnitude, caused partly by the structure of the epi- and endocardium and partly by inhomogeneous staining and lighting of the tissue). To overcome this obstacle, the raw data needs to be processed before measurements of activated areas and activation times can take place.

\footnotetext{
${ }^{10}$ MultiRecorder, written mainly by Johannes Schröder-Schetelig
} 


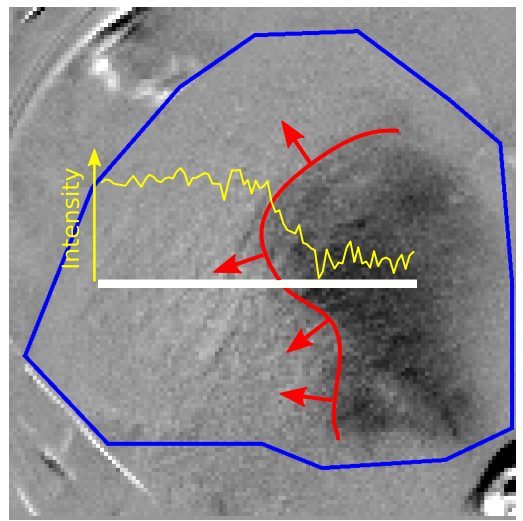

FiguRE 4.19: A single frame from an optical mapping experiment (intact dog heart) during normal sinus rhythm. For this image, the long time median was subtracted for each pixel. The approximate silhouette of the heart is given in blue, the wave front is delineated in red. The pixel intensity values along the white line are shown in yellow, excited tissue corresponds to darker values. Noise artifacts are still clearly visible, the only preprocessing for this image was to subtract the temporal median from each pixel.

As described in Luther et al. [45] and in more detail in Bittihn [82, the following processing steps were performed in order to obtain the activation times:

- A spatial and temporal box filter $\left(5 \mathrm{pixel}^{2} \times 5 \mathrm{pixel}^{2}, 3\right.$ frames $)$ was applied to the data to remove noise.

- Each pixel's time series was normalized locally with respect to its minimum and maximum onto the range $[0,1]$.

- A simple threshold, typically at 0.5 , was applied to classify the pixels' states as active or inactive.

- To remove erroneous activation detection due to noise, only those pixels counted as active (normalized intensity below the threshold) where the average of the next 10 frames also was below the threshold.

- Only pixels whose intensity changes were higher than the noise level and who were located within one heart chamber (e.g. the right atrium in figure 4.20) were taken into account.

- Under these transformations, the activation time $T(\mathbf{x})$ for each pixel $\mathbf{x}$ is the first time when its state changed from inactive to active.

Figure 4.20 shows an activation map obtained with this method, where the time between pulse delivery and activation of each tissue pixel is shown graphically over an unprocessed image taken by the camera. 


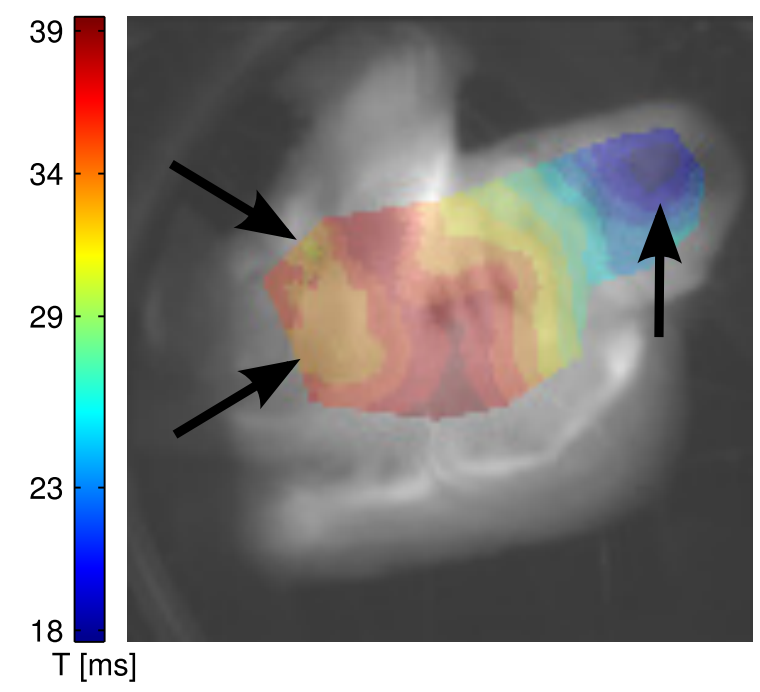

FIGURE 4.20: The activation map of an piece of atrial/ventricular canine dog heart, under an external electric field of approximately $0.22 \frac{\mathrm{V}}{\mathrm{cm}}$. Overlayed over the original grayscale image of the tissue are the color coded times (after onset of the stimulating pulse) at which the tissue became activated. Image courtesy of Philip Bittihn [82]
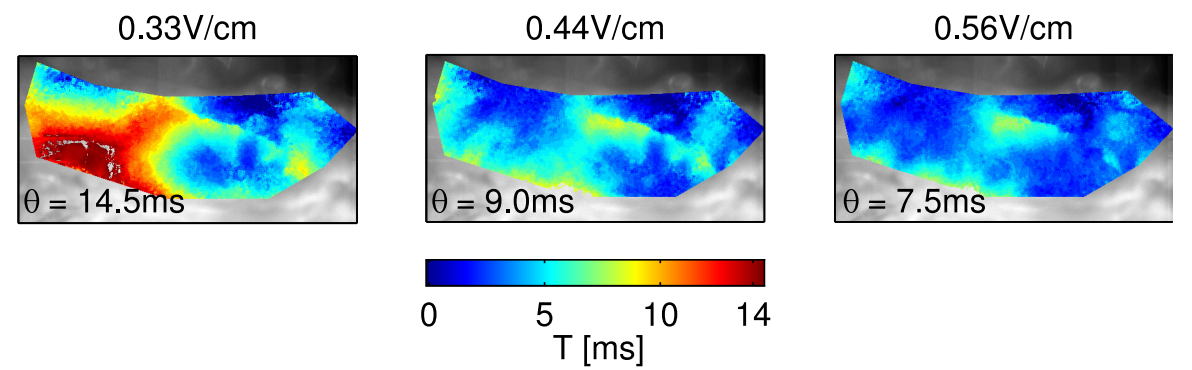

Figure 4.21: Activation times in the canine heart under external electric fields. The required time to activate the whole tissue decreases with increasing field strength, note that the temporal resolution here is limited by the image acquisition rate of approximately $2 \mathrm{~ms} /$ frame. Images courtesy of Philip Bittihn [82]

Following this calculation of activation times for each single pixel, we obtained the activation time $\tau$ for a bulk volume as follows. For each applied pulse of field strength $E$, the activation time $\tau(E)$ was defined as the time between the activation of 1 and $99 \%$ of the tissue [45, 82].

\subsubsection{Activation Times in Cardiac Tissue}

As expected, the activation time decreases with increasing field strength, as can be seen in figure 4.21, where the activation times are plotted against the field strength, for atrial and ventricular preparations. Common to all the measured activation time curves $\tau(E)$ is an apparent power law behavior:

$$
\tau(E) \propto E^{\beta} \text { with } \beta<0
$$




\subsubsection{Linking Times and Sizes}

Although others [62 have shown how certain vascular features are related via their scaling exponents (see also section 4.2.1 on p. 28), there have been no statements about the sizes or activation times before. Nevertheless it seems reasonable that there should at least be a prediction about the exact relation between $\beta$ and $\alpha$ (the exponent in $\operatorname{Pr}(r) \propto r^{\alpha}$ ). After all, the density of blood vessels (or heterogeneities) of a given size is not only governed by a power law relation as shown in section 5.1.1. but via equation (4.6) it also influences the number of wave sources and therefore (with 4.16) the activation time.

Following the arguments laid out in Luther et al. [45] and Bittihn [82, let us assume sufficiently homogeneously distributed heterogeneities of size $r$ in the tissue, whose size distribution follows the power law

$$
\begin{gathered}
\operatorname{Pr}(r) \propto r^{\alpha} \\
\text { with } \quad \alpha<-1
\end{gathered}
$$

and which act as obstacles in the sense of section 4.1.1. It makes sense to assume that these heterogeneities are rather small so the assumption about homogeneous distribution is most likely true. Since for small obstacle sizes according to 4.6 the electric field required to emit a wave behaves like

$$
R_{\min } \propto \frac{1}{E}
$$

A.3 from the appendix.)

the density $\rho \equiv \frac{N}{V}$ of wave emitting heterogeneities (WEHs) in the tissue should be:

$$
\begin{aligned}
\rho(E) & =\rho\left(R_{\min }(E)\right) \equiv \frac{N\left(r \geq R_{\min }\right)}{V}=\frac{1}{V} \int_{R_{\min }}^{\infty} N(r) \mathrm{d} r \\
& \propto \int_{R_{\min }}^{\infty} \operatorname{Pr}(r) \mathrm{d} r \propto \int_{R_{\min }}^{\infty} r^{\alpha} \mathrm{d} r=\left[\frac{1}{\alpha+1} r^{\alpha+1}\right]_{R_{\min }}^{\infty} \\
& \stackrel{4.19}{=} 0-\frac{1}{\alpha+1} R_{\min }^{\alpha+1} \\
\Longrightarrow \quad \rho(E) & \propto R_{\min }^{\alpha+1} \frac{\mathrm{A} \cdot 3}{\alpha} E^{-(\alpha+1)}
\end{aligned}
$$


Now applying this result to the activation time dimensional argument 4.16) yields for a physical tissue dimension $D$ :

$$
\begin{array}{cl}
\stackrel{\stackrel{(4.16)}{\longrightarrow}}{\longrightarrow} \tau(E) \propto \rho(E)^{-\frac{1}{D}} \propto E^{\frac{\alpha+1}{D}} \\
\text { together with } \tau(E) \propto E^{\beta}(\text { from 4.17) } \\
\Longrightarrow \beta=\frac{\alpha+1}{D}
\end{array}
$$

Using equations (4.21) and (4.24), it is possible to transfer the size statistics into activation times and vice versa. This also applies to the formulation in terms of power law distributions (lower part of the diagram).

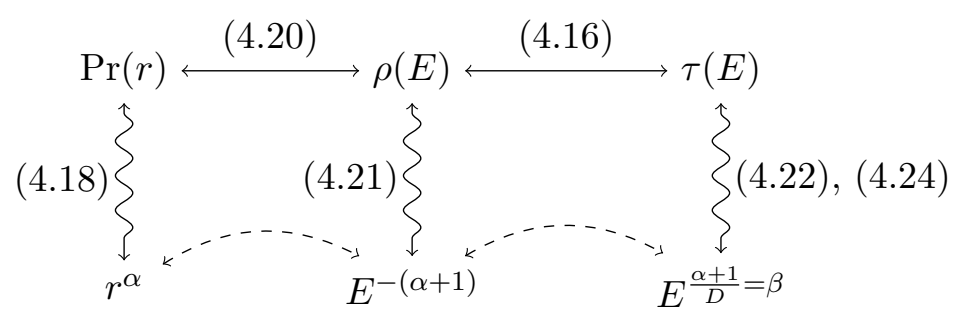





\section{Chapter 5}

\section{Heterogeneity Characterization Results}

\subsection{Size Distributions of Blood Vessels}

\subsubsection{Canine Blood Vessel Size Distributions}

The identified vessel sizes in the examined dog hearts ranged from the order of the voxel resolution (approximately $50 \mu \mathrm{m}$ ) to the size of the largest artery present in the volume or the catheter, when it could not be completely removed. Between these two extremes, the distribution of vessel sizes apparently follows a power law, as is shown in two examples in figure 5.1. Only towards very large sizes, one can see a deviation due to finite sample sizes, the largest artery or the catheter become very prominent. Apart from this artifact, the sizes show a consistent behavior over the whole expected range.

The size distributions for all examined heart tissues can be found in detail in the appendix, in section B.1.

For all analyzed scans, I fitted a power law curve $\operatorname{Pr}(r)=a \cdot r^{\alpha}$ to the data, using a least-squares algorithm implemented in the graphical data analysis tool Grace [90]. The obtained exponents $\alpha$ are given in detail in table 5.1 and plotted as box plots in figure 5.2 . In this series of experiments, no significant difference between the exponents in canine atria and ventricles can be found (according to Welch's $t$ test, the null hypothesis of equal means can explain the results with $p=0.83)$. 


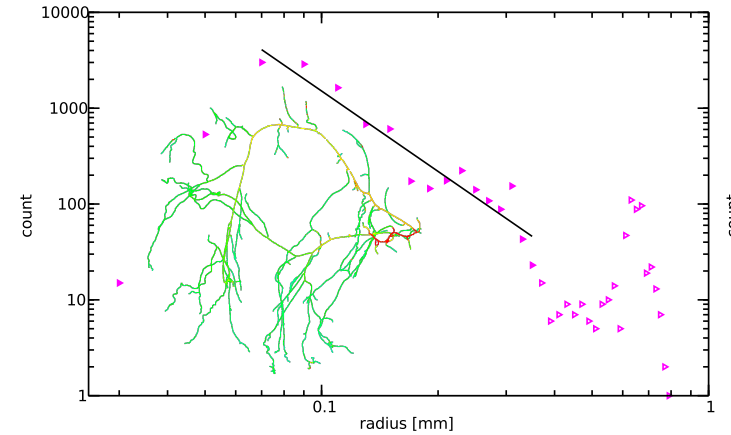

(a) canine atrium

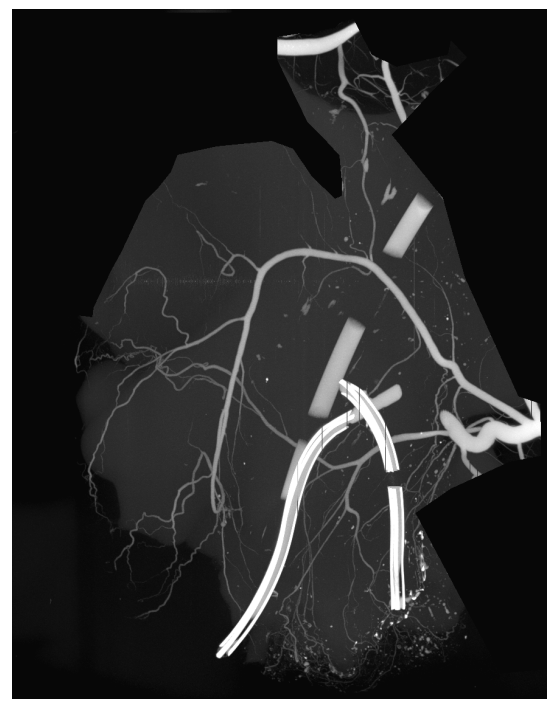

(c) atria: $\mu \mathrm{CT}$ projection

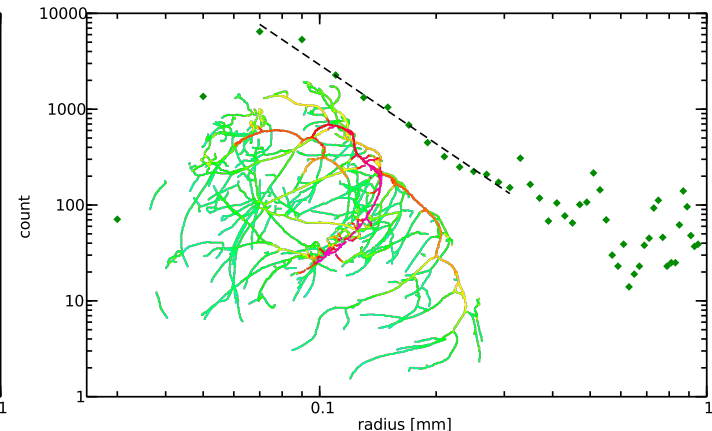

(b) canine ventricle

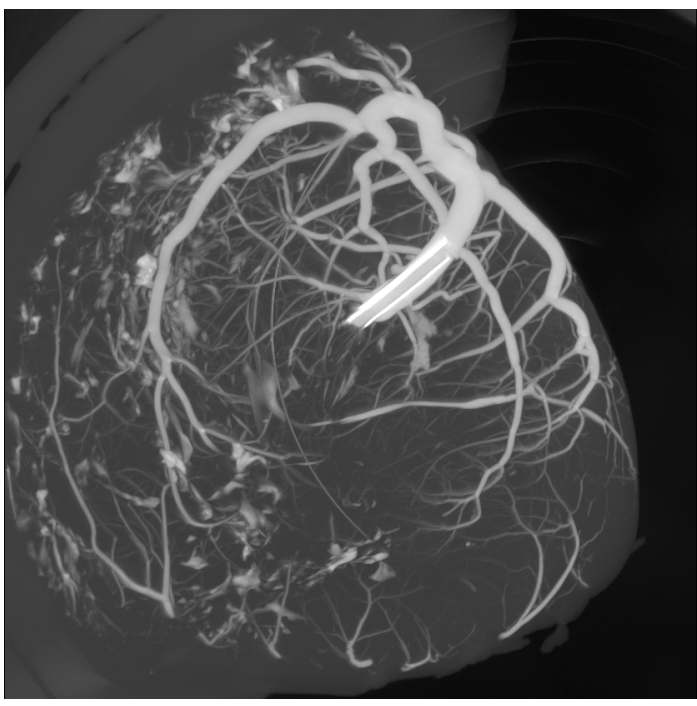

(d) ventricle: $\mu \mathrm{CT}$ projection

Figure 5.1: Vascular size distributions in dog hearts: Overlayed over the raw radius counts $((\mathrm{a})$ and $(\mathrm{b}))$ are the color-coded positions of the detected blood vessels in a maximum intensity projection (MIP). The power law of the size distributions is clearly visible in the expected range. A line representing the fitted power law exponent is overlayed in both plots. For comparison, (c) and (d) show a MIP of the $\mu \mathrm{CT}$ data that was used for the size reconstructions. Figure first published in [45].

\begin{tabular}{l|l} 
Preparation & $\alpha \pm$ std. error \\
\hline \hline$\# 1$ & $-2.80 \pm 0.23$ \\
$\# 2$ & $-2.74 \pm 0.41$ \\
$\# 3$ & $-2.76 \pm 0.17$ \\
$\# 4$ & $-2.67 \pm 0.15$ \\
$\# 5$ & $-2.79 \pm 0.25$ \\
\hline average & $-2.74 \pm 0.05$
\end{tabular}

(a) canine atria

\begin{tabular}{l|l} 
Preparation & $\alpha \pm$ std. error \\
\hline \hline$\# 1$ & $-2.49 \pm 0.11$ \\
$\# 2$ & $-2.73 \pm 0.10$ \\
$\# 3$ & $-3.09 \pm 0.13$ \\
\hline average & $-2.75 \pm 0.30$
\end{tabular}

(b) canine ventricles

TABLE 5.1: Exponents for the power law scalings of cardiac blood vessel size distributions in adult beagle dogs. 


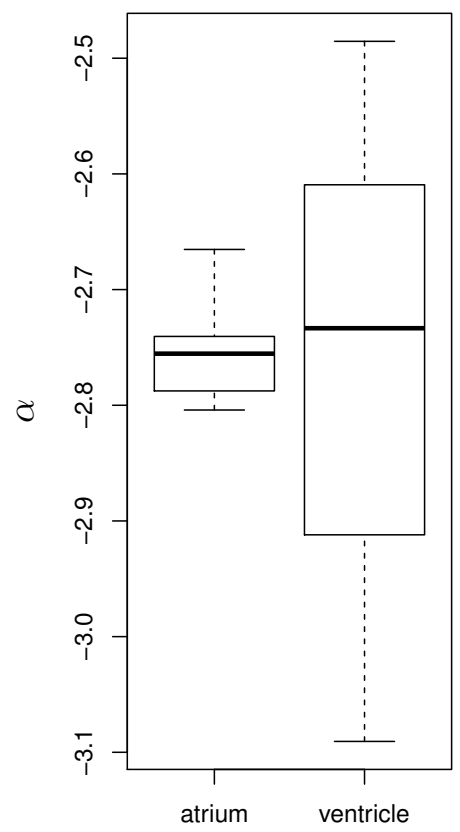

Figure 5.2: Box plot of the scaling exponents $\alpha$ for canine atria (left, $N=5$ ) and ventricles (right, $N=3$ ). The boxes mark the quartiles, the thick lines indicate the median of each group. According to this analysis, the scaling exponents for ventricles and atria are not significantly different $(p>0.5)$.

\subsubsection{Porcine Blood Vessel Size Distributions}

Although the voxel resolution of the reconstructed pig heart volumes was $60 \mu \mathrm{m} /$ voxel, the edge response function of the $\mathrm{\mu CT}$ had, depending on the exact scanning setup, a width of around $200 \mu \mathrm{m}$ for an intensity rise from 10 to $90 \%$ full intensity. This corresponds well to the smallest detected vessel sizes of approximately $100 \mu \mathrm{m}$ in this sample.

As with the dog hearts, the pig vessel sizes were analyzed and a power law distribution $\operatorname{Pr}(r) \propto r^{\alpha}$ was fitted to the data (from the maximum to the minimum before the last peak), a typical result for the size distribution is shown in figure 5.3

The exponents $\alpha$ which obtained from these examinations (detailed distributions are plotted in the appendix, section B.1.2 are as follows:

\begin{tabular}{l|l} 
Preparation & $\alpha \pm$ std. error \\
\hline \hline$\# 1$ & $-2.54 \pm 0.34$ \\
$\# 2$ & $-2.88 \pm 0.15$ \\
$\# 3$ & $-2.94 \pm 0.22$ \\
$\# 4$ & $-3.91 \pm 0.33$ \\
$\# 5$ & $-4.63 \pm 0.12$ \\
$\# 6$ & $-3.79 \pm 0.28$ \\
$\# 7$ & $-2.86 \pm 0.19$ \\
\hline average & $-3.47 \pm 0.76$
\end{tabular}




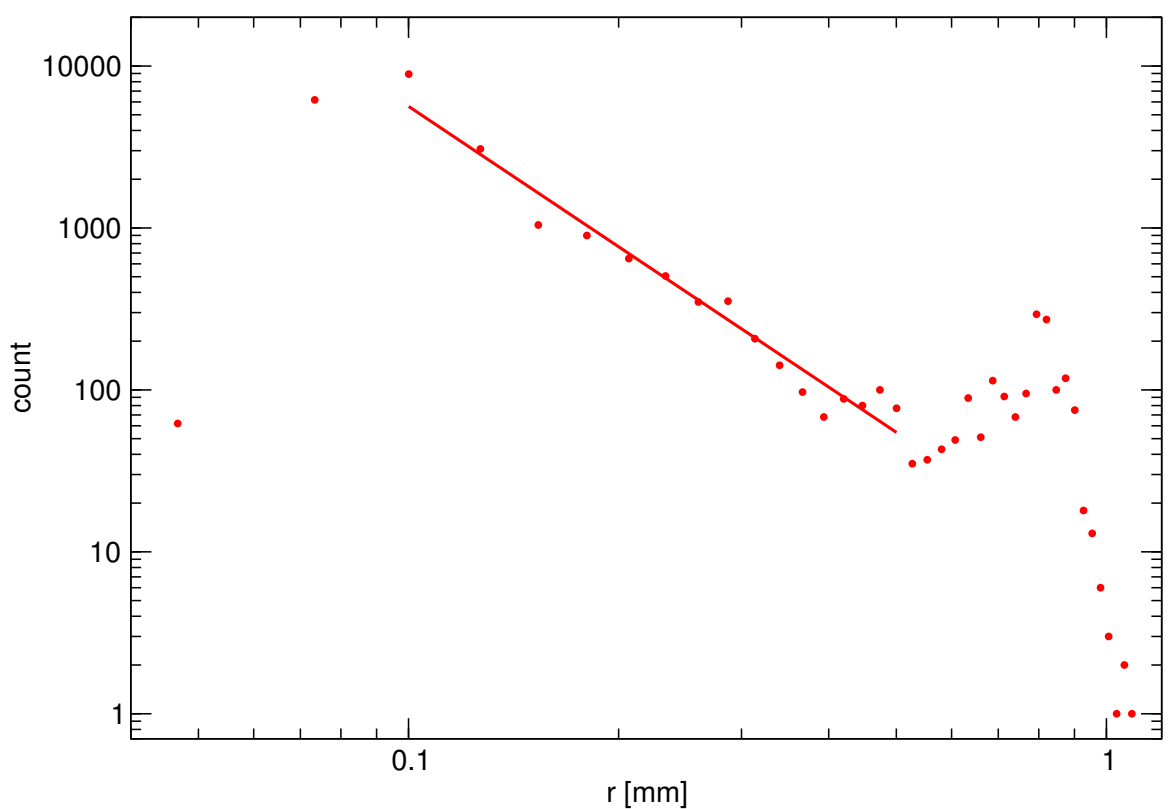

Figure 5.3: Size scaling in a porcine heart. The line represents the fitted power law $\operatorname{Pr}(r)=7.43\left(\frac{r}{\mathrm{~mm}}\right)^{2.88}$. The peak around $0.8 \mathrm{~mm}$ corresponds to the largest arterial branch, the RCA. The voxel resolution of the $\mu \mathrm{CT}$ reconstruction was $60 \mu \mathrm{m}$.

These exponents show higher variations compared to the ones yielded for the dog hearts, which may be because the heart were prepared longer after the experiment or owing to the different contrast agent.

\subsubsection{Vascular Size Distributions from Other Sources}

I applied 4.13) to the numbers for the RCA, LAD and $\mathrm{LCx}^{1}$ arterial branches published in Kassab et al. [65, tables 1-3 and 9]. The obtained size distributions are shown in figure 5.4 , the resulting exponents $\alpha$ are:

\begin{tabular}{c|c|c} 
tissue & $\alpha$ & \multicolumn{1}{|c}{$\alpha_{2}$} \\
\hline \hline $\mathrm{LAD}$ & $-1.01(6)$ & $-1.29(13)$ \\
$\mathrm{LCx}$ & $-0.88(7)$ & $-0.85(9)$ \\
$\mathrm{RCA}$ & $-1.00(5)$ & $-1.29(6)$
\end{tabular}

The original data was obtained from polymer corrosion casts (larger vessel diameters) and extrapolated from histological slices (smaller diameters). The exponents $\alpha$ were fitted over the whole range, $\alpha_{2}$ only over the larger vessels.

These numbers should be taken with a grain of salt, though, the assumption that taking the mean lengths and diameters returns representative results may turn out to be

\footnotetext{
${ }^{1}$ right coronary artery, left anterior descending and left circumflex
} 


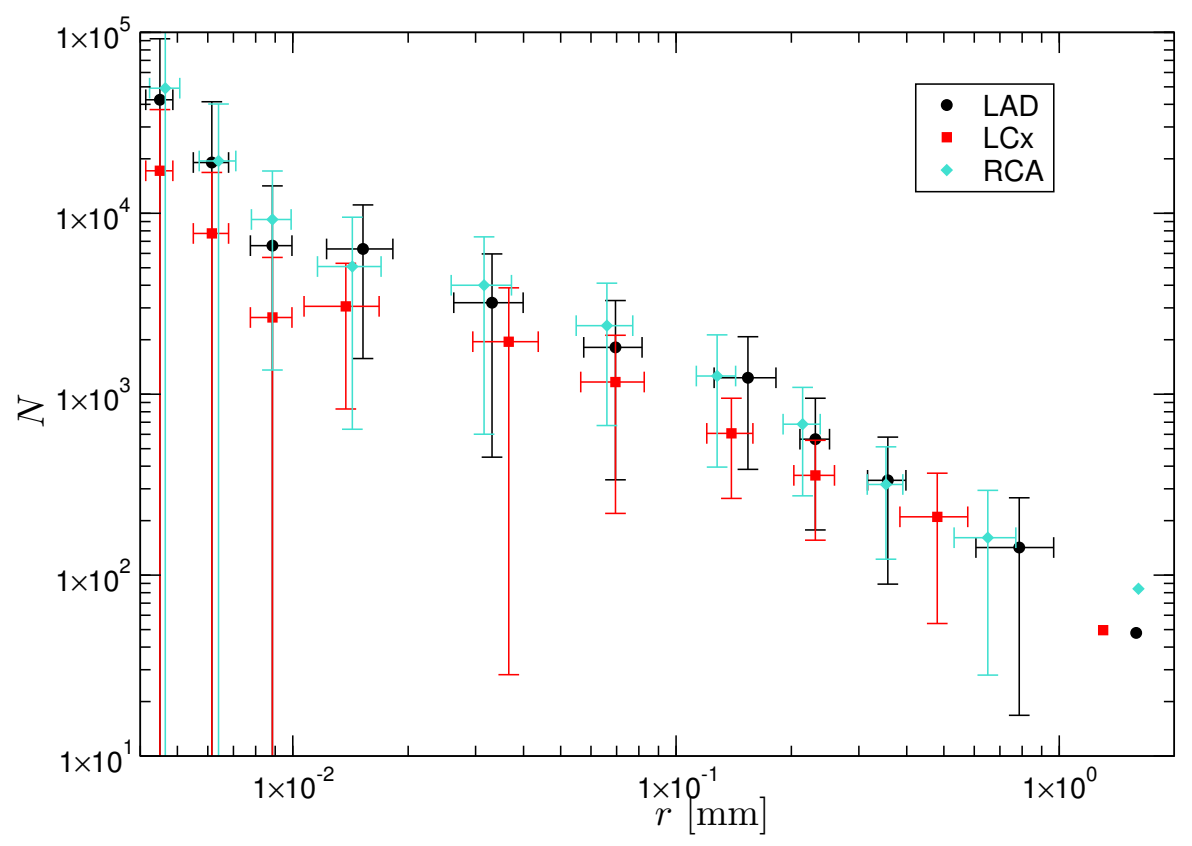

FiguRE 5.4: Size scaling in pig coronary arteries from vascular morphometric data as published in [65]. Each data point corresponds to one hierarchical vessel order, starting with order 1 from the left. Note how the error bars allow for a wide range of possible power law exponents $\alpha$ which would be consistent with this data. Below order 5-6 $\left(r<1 \times 10^{-1} \mathrm{~mm}\right)$, the data was sampled mostly from histological slices, higher orders come mainly from macroscopic polymer corrosion casts of the vasculature. Colors and symbols denote for which part of the vasculature the measurements were made: Black circle $(\bullet)$ : LAD, red square $(\boldsymbol{\bullet})$ : LCx, cyan diamond $(\bullet)$ : RCA.

wrong. The large uncertainties associated with the original data (see the error bars in figure 5.4 are caused by three factors: The size of vessel elements of the same order varies considerably, the number of elements in an order is only extrapolated from small tissue samples for smaller orders, and the two different measurement methods (corrosion casts and histological slices) may introduce an additional bias. Therefore, fitting a power law distribution to only the larger sizes (orders 7-10) results in the steeper exponents $\alpha_{2}$ from the table above.

\subsubsection{Comparison of Vascular Size Distributions of Different Origin}

Figure 5.5 shows the distribution of the size scaling in pig and in dog hearts (from table 5.1) and the values $\alpha_{2}$ calculated from independent branch scaling values in the literature. $\alpha_{\text {pig }}$ and $\alpha_{\text {dog }}$ seem to be different, but their means do not differ statistically significantly, possibly due to the small sample sizes. 
Figure 5.5: Box plot of the size exponents in porcine and canine hearts. The three single values around -1 are the $\alpha_{2}$ as described in section 5.1 .3 they are significantly different from the ones measured directly from $\mu \mathrm{CT}$ data $(p=0.0001)$. Although the $\alpha$ distribution of pig and $\operatorname{dog}$ hearts are different, this difference is not statistically significant $(p>0.05)$.

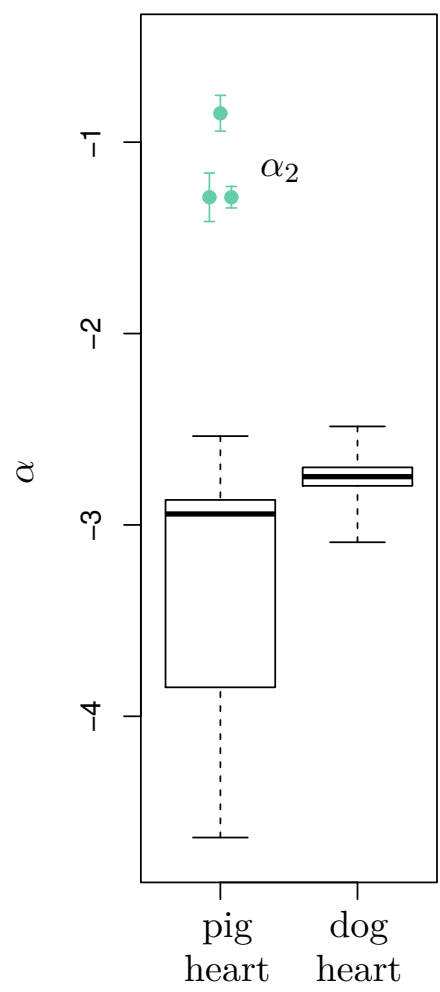

\subsection{Activation Times}

\subsubsection{Activation Time Exponents}

Philip Bittihn [45, 82] analyzed the optical mapping data of dog hearts to yield activation times as a function of the applied field strength, $\tau(E)$. Least-squares fits of equation (4.17) to this data yielded exponents $\beta$ with generally small variations as listed in table 5.2 and figure 5.6. As the vascular size distribution exponents, the exponents from these data sets do not show a significant difference between atria and ventricles.

\subsubsection{Size Distributions Linked to Activation Times}

Applying the power law relations from section 4.6 .2 to the measured data yields figure 5.7. with $D=3$ for ventricular and $D=2$ for atrial data (for a sparse enough distribution of WEHs, the atrial myocardium is thin enough to be considered essentially two-dimensional). When the power law relations re applied to the exponents from table 5.1. we obtain the following predicted exponents, which are within one standard error of the measured values from table 5.2 .

\begin{tabular}{l|c|r|r} 
Preparation & $\alpha$ & predicted $\beta$ & measured $\beta$ \\
\hline \hline canine atria $(D=2)$ & $-2.74 \pm 0.05$ & $-0.87 \pm 0.025$ & $-0.75 \pm 0.18$ \\
canine ventricles $(D=3)$ & $-2.75 \pm 0.30$ & $-0.58 \pm 0.10$ & $-0.81 \pm 0.23$
\end{tabular}




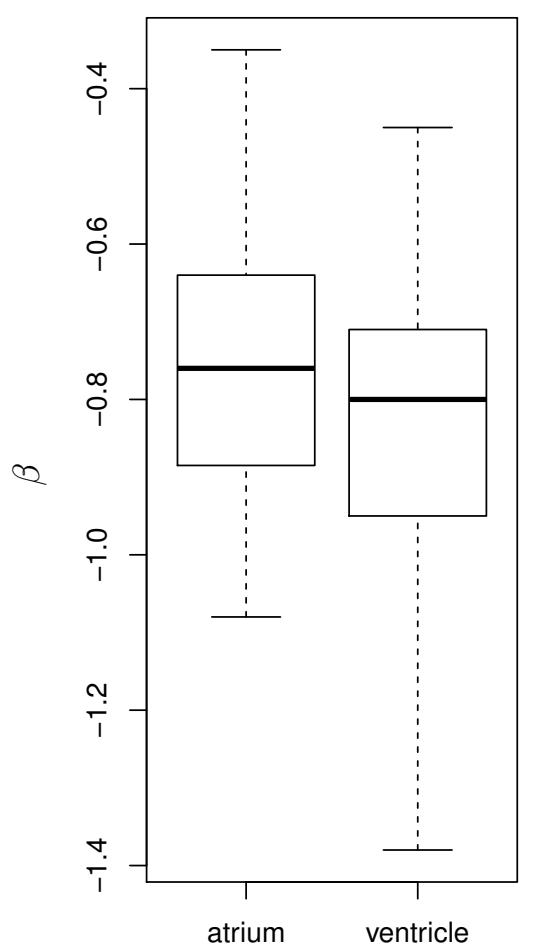

Figure 5.6: Box plot of the activation time scaling exponents $\beta$ for canine atria (left, $N=23$ ) and ventricles (right, $N=17$ ). The boxes mark the quartiles, the thick lines indicate the median of each group. According to this analysis, the scaling exponents for ventricles and atria are not significantly different $(p>0.3)$.
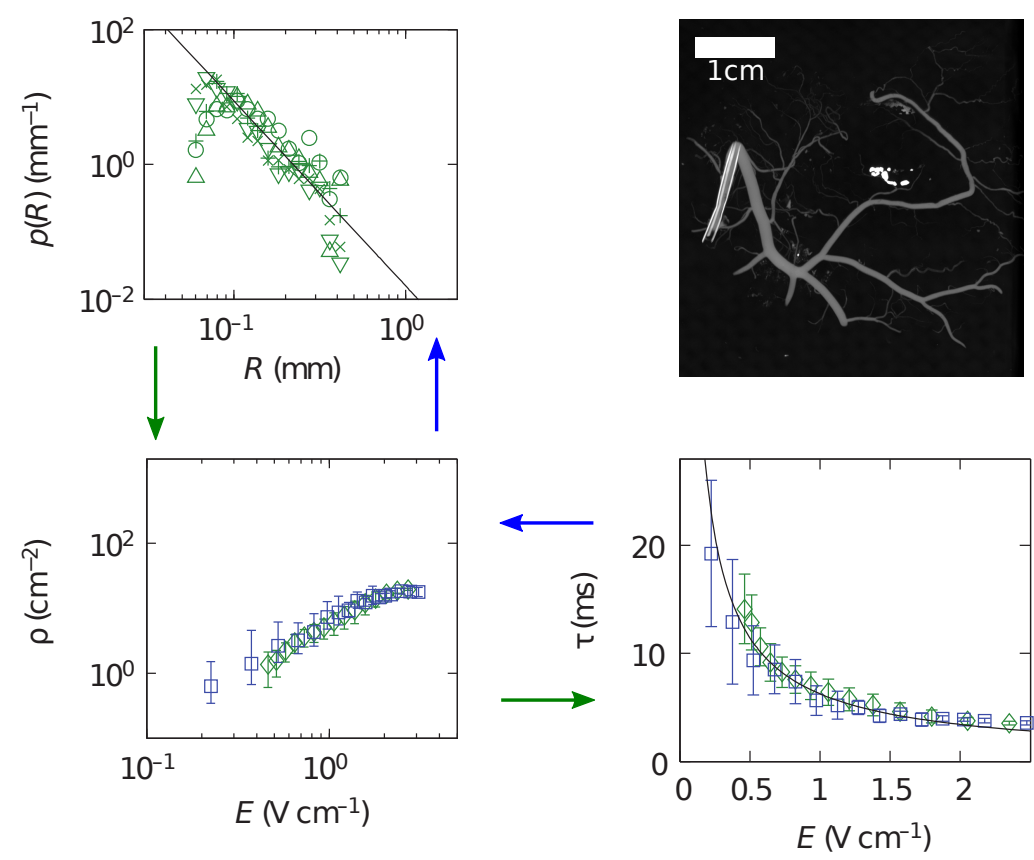

FiguRE 5.7: Vascular size distributions and activation times in dog atria, transformed using 4.25). The green symbols stand for the data which originates in the size distribution (upper left panel) and is being transformed into WEH source densities (lower left) and activation times (lower right). The original activation time data, reversely, is represented by the blue symbols and can be transformed into a corresponding size distribution. This figure was first published in [45]. 


\begin{tabular}{l|l} 
Preparation & $\beta$ (mean \pm std. error) \\
\hline \hline$\# 1$ & $-0.76 \pm 0.09$ \\
$\# 1$ & $-0.78 \pm 0.06$ \\
$\# 2$ & $-0.35 \pm 0.10$ \\
$\# 2$ & $-0.97 \pm 0.10$ \\
$\# 3$ & $-1.08 \pm 0.41$ \\
$\# 4$ & $-0.92 \pm 0.06$ \\
$\# 4$ & $-0.54 \pm 0.08$ \\
$\# 4$ & $-0.94 \pm 0.07$ \\
$\# 4$ & $-0.68 \pm 0.08$ \\
$\# 4$ & $-0.90 \pm 0.07$ \\
$\# 4$ & $-0.55 \pm 0.07$ \\
$\# 5$ & $-0.67 \pm 0.08$ \\
$\# 5$ & $-0.55 \pm 0.05$ \\
$\# 5$ & $-0.81 \pm 0.12$ \\
$\# 5$ & $-0.67 \pm 0.07$ \\
$\# 5$ & $-0.83 \pm 0.09$ \\
$\# 5$ & $-0.64 \pm 0.03$ \\
$\# 6$ & $-0.99 \pm 0.07$ \\
$\# 6$ & $-0.53 \pm 0.09$ \\
$\# 6$ & $-0.87 \pm 0.10$ \\
$\# 6$ & $-0.67 \pm 0.09$ \\
$\# 6$ & $-0.80 \pm 0.08$ \\
$\# 6$ & $-0.64 \pm 0.10$ \\
\hline average & $-0.75 \pm 0.18$
\end{tabular}

\begin{tabular}{l|l} 
Preparation & $\beta($ mean \pm std. error $)$ \\
\hline \hline$\# 1$ & $-0.80 \pm 0.06$ \\
$\# 1$ & $-1.38 \pm 0.09$ \\
$\# 1$ & $-0.45 \pm 0.11$ \\
$\# 1$ & $-0.98 \pm 0.10$ \\
$\# 2$ & $-0.77 \pm 0.06$ \\
$\# 2$ & $-0.81 \pm 0.05$ \\
$\# 2$ & $-0.95 \pm 0.08$ \\
$\# 2$ & $-0.80 \pm 0.08$ \\
$\# 2$ & $-1.01 \pm 0.08$ \\
$\# 2$ & $-1.12 \pm 0.10$ \\
$\# 3$ & $-0.89 \pm 0.05$ \\
$\# 3$ & $-0.76 \pm 0.05$ \\
$\# 3$ & $-0.71 \pm 0.05$ \\
$\# 3$ & $-0.75 \pm 0.03$ \\
$\# 3$ & $-0.58 \pm 0.03$ \\
$\# 3$ & $-0.56 \pm 0.04$ \\
$\# 3$ & $-0.53 \pm 0.03$ \\
\hline average & $-0.81 \pm 0.23$
\end{tabular}

(b) canine ventricles

(a) canine atria

TABLE 5.2: Measured exponents and weighted averages for the power law exponent $\beta$ of cardiac activation times $\tau(E) \propto E^{-\beta}$ in adult beagle dogs. For each preparation, several activation time measurements were made and their weighted average was calculated. Data was first published in [45. 


\section{Chapter 6}

\section{In Vivo LEAP Experiments}

In clinical application, life-threatening ventricular fibrillation (VF) is usually treated with electrical shocks resulting in field strengths of over $5 \frac{\mathrm{V}}{\mathrm{cm}}$ (if delivered externally, this means energies of $150 \mathrm{~J}$ which at typically $15 \mathrm{~ms}$ corresponds to over $20 \mathrm{~A}$ at about $500 \mathrm{~V}$ [91]). For patients at an increased risk of ventricular fibrillation, implantable cardioverter-defibrillators (ICD) are a common measure, with over 200000 new implants taken place in 2009 alone [92. An ICD typically delivers shocks with energies of about $40 \mathrm{~J}$ in the case of detected $\mathrm{VF}$, which is considerably less than for external defibrillation but can nevertheless lead to traumatic pain in the case of malfunctions [38] (when the patient is still conscious). Because of its potential life-saving abilities, there is currently no alternative to ICDs, so lower energy alternatives such as LEAP (see chapter 3 for an introduction) are an area of interest. In order to advance these new methods towards clinical applicability, it is necessary to test them in models that are as close to human use cases as possible.

\subsection{Previous Studies}

After the first theoretical proposals [47], LEAP has been experimentally tested in numerous systems: In cell cultures of cardiomyocytes [93], in perfused rabbit hearts [94] and dog heart tissue preparations [45] and in vivo in dog hearts [45].

In [93] Pumir et al. argue that for neonatal rat myocyte cell cultures, as predicted in [47, the required energy to unpin a pinned spiral from a non-conducting heterogeneity should be a factor of 500 smaller than the energy for complete defibrillation.

We showed in [45] that in dog heart preparations it is possible to decrease the energy required to terminate VF to $15 \%$ of the single shock defibrillation energy. For atrial 
fibrillation (AF), the necessary energy was only $10 \%$, both in ex vivo preparations and in in vivo experiments.

\subsection{Towards Clinical Application}

\subsubsection{Experimental Criteria}

In order to further develop LEAP towards clinical application, model systems need to be as close to the human organism as possible. This includes:

Physiological Environment Although many insights into the mechanisms of cardiac activation, pathological excitation patterns and termination of e.g. fibrillation can be gained through experiments with cell cultures or ex vivo preparations, these methods always include a certain level of abstraction from living organisms. Only with in vivo models there is a realistic chance of capturing all relevant properties and responses to defibrillation attempts.

Scaling In order to develop fibrillatory excitation patterns comparable to the human heart, the ratio of the excitable substrate's size to the typical excitation wave lengths should be about the same as in human hearts. In general, Strain and Greenside [95] showed than turbulent spatio-temporal behavior requires a minimum medium size. In too small media such as mouse or rabbit hearts, fibrillatory patterns are enabled through administration of drugs which affect the action potential. For example, acetylcholine is commonly used, since it shortens the action potential [96] and thus promotes arrhythmia.

Field geometry The most relevant potential application for LEAP would be in ICDs (implantable cardioverter-defibrillators). The common implementation of one intraventricular coil as one electrode and the case of the ICD itself, implanted below the pectoralis major muscle, as the other electrode, leads to a very specific field geometry which is not reproduced in most experiments so far. In most numerical simulations, cell culture experiments [97], tissue preparations and even many in vivo experiments [45] though, the electric field is mostly homogeneous. This difference must be taken into account to compare results.

Species Results obtained for a single species only may not giove the full picture yet. E.g. the Purkinje fiber network is different in pigs compared to humans [98] and the different orientation of the heart relative to the chest may play a role in $\mathrm{VF}$ termination through external shocks. Deviating shapes of action potentials in 
other species indicate differences on the cellular level and in the process of turbulent activity, which may also make the transfer from animal models to humans challenging.

\subsubsection{Clinically Relevant Animal Models for LEAP}

Previous experiments assessing the viability and efficiency of LEAP in whole hearts only included animals up to (regarding the size) rabbit [94]. In dog hearts, LEAP was tested only in atria or in isolated tissue, which changes both the size of the relevant medium and also potentially its topology. To evaluate LEAP in models closer to clinical application in humans, we conducted a series of in vivo experiments with pigs.

The hearts of young or small pigs are comparable in size to human hearts (approximately $210 \mathrm{~g}$ for pig hearts [99] compared to about $300 \mathrm{~g}$ in humans [27]), as well as the dimensions of the thorax, which allowed us to test electric configurations similar to the ones used in ICD patients. The results showed that the required energy for defibrillation was about the same as applied on humans.

We used pigs of two races for our LEAP experiments: juvenile male German Landrace animals and adult Göttingen minipigs. The pigs of both races were comparable in body weight in heart size. We expected this diversity to highlight possible biasing effects due to age, while still retaining a model that is comparable to adult humans.

Since the previous most successful attempts at testing LEAP in experiments were done using beagle dogs (though only $\mathrm{AF}$ in vivo and heart slices ex vivo), we decided to continue with that animal model and do in vivo VF and whole heart ex vivo experiments. Here the in vivo experiments were done with with epicardial mesh electrodes in an open chest setting instead of ICD catheter and can.

\subsection{The Experimental Setup}

The experiments were set up so that a number of constraints would be fulfilled:

- Reproducible inducibility of fibrillation with very low energies.

- Reliable termination of VF even if LEAP fails in order to minimize the duration of ischemia for the heart.

- Manually controllable and reproducible delivery of shocks to the heart, either multiple shocks for LEAP or single shocks for comparison with classical defibrillation. 


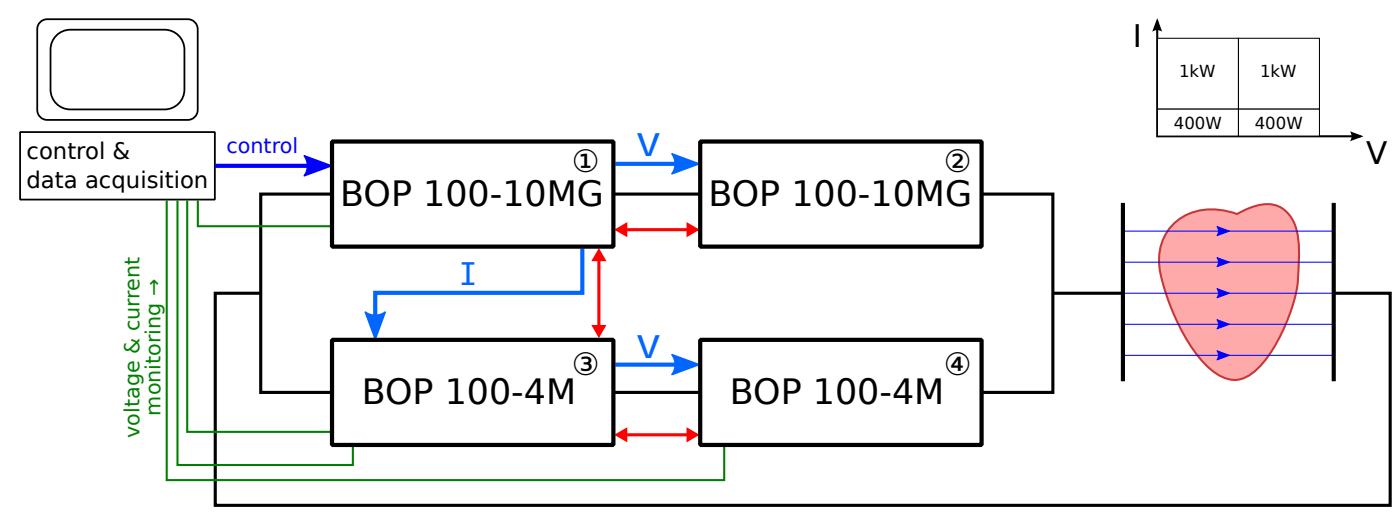

Figure 6.1: Schematic of four Kepco power amplifiers connected in series and parallel, for defibrillation of the heart in the experiment (on the right). The analog BOP 100-4M deliver up to $100 \mathrm{~V}$ and $4 \mathrm{~A}$, while the digital BOP 100-10MG can support currents up to $10 \mathrm{~A}$ at the same voltage. Together they can defibrillate with $200 \mathrm{~V} \times 14 \mathrm{~A}$ (power diagram in the upper right corner). In this schematic, blue lines depict the control hierarchy. The computer controls the amplifier (1)'s output, which in turn passes on its current state to (2) through (4) for voltage and current amplification, respectively. The amplifiers are connected by fail-safe emergency shut-off signal lines (red) so that failure in one device shuts off the others as well. The current and voltage of the devices is continuously monitored by the computer (green lines). Further explanations are given in the text below.

The single shocks should be strong enough (i.e. be above the defibrillation threshold (DFT)) to reliably defibrillate the heart.

- Ability to stimulate the heart should it go into asystole after successful defibrillation.

- Acquisition of the relevant electrophysiological data as collected by ICDs and beyond, such as ECG, intraventricular action potential and defibrillation current.

- Controlled life support for the animal to attain reproducible results over the course of the experiment and to minimize the effects of the unavoidable periods of relative ischemia during VF.

\subsubsection{Prototype LEAP Devices}

According to the specific needs, we used two distinct kinds of power supplies for defibrillation. Common to both are the "connections" to the animal mimicking clinical application. 


\subsubsection{Power Amplifiers for Lower Energy Requirements and Higher Flex- ibility}

In the open chest experiments we conducted on beagle dogs, the required peak power was low enough that commercially available high-power amplifiers were sufficient. We used up to four amplifiers (two BOP 100-4M, $400 \mathrm{~W}$, two BOP 100-10MG, $1 \mathrm{~kW}$, Kepco Inc., USA) together delivering a maximum power of $2800 \mathrm{~W}$, or $200 \mathrm{~V} \times 14 \mathrm{~A}$. Although the amplifiers themselves were available off the shelf, connecting them in series and in parallel as shown in figure 6.1 still needed major adjustments. Specifically, this included:

Synchronization The amplifiers can act either as current or as voltage sources (or sinks, depending on the voltage across it load outputs). In order to deliver the needed current to the heart, all four devices must work together, and especially their polarity must be matching lest they act as a short-circuit with positive feedback. The $1 \mathrm{~kW}$ amplifier marked with (1) in figure 6.1 acted as the primary master to the other amplifiers. It was controlled directly through a $\mathrm{DA} / \mathrm{AD}$ converter by a custom LabView program (see figure 6.8 for a screenshot of the defibrillator control software). Information about the voltage across its load outputs was fed into the primary slave (2), an identical device which effectively doubled the voltage. To ensure that no current was flowing circularly through the four amplifiers, the secondary master (3) was configured to act as a current source providing $40 \%$ of the current flowing through (1). This $400 \mathrm{~W}$ amplifier in turn worked in series with another identical device, the secondary slave (4) in voltage mode.

This configuration guaranteed that all amplifiers worked synchronously at the same level of their respective maximum power. The control flow is shown with blue arrows between the amplifiers in the figure.

Safety Because the amplifiers were set to reach their given target voltage/current with all the power available, one faulty device might cause a cascade of potentially harmful or damaging failures in the others as well. Therefore we connected all amplifiers with a chain of fail-safe, partly custom-built shut-off triggers, denoted with red lines in figure 6.1. In the case of one device detecting a malfunction and shutting itself off, all other devices would switch themselves off immediately as well.

Recording In order to reliably record the energy which is actually delivered to the heart, the output current and voltage of the involved amplifiers was fed back into the control and measurement computer, shown as green lines in the setup schematic. 
Figure 6.2: The power amplifiers in a 19 " rack. Correspondence of single amplifier units to figure 6.1 is denoted by the numbers (1)... (4).

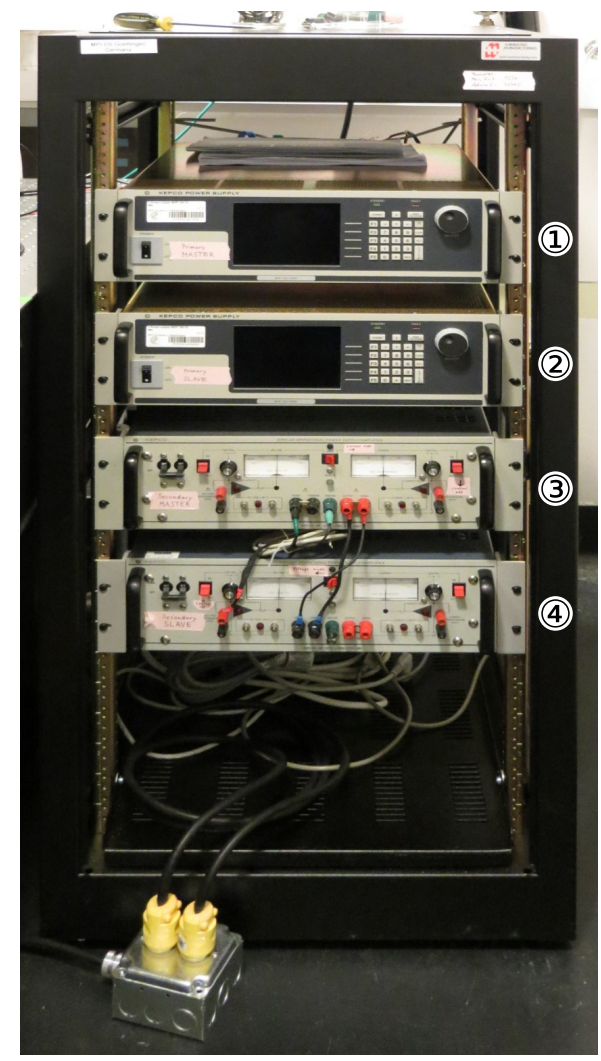

This setup allowed us to adjust e.g. the waveform of the LEAP pulses arbitrarily and also to deliver an arbitrary number of pulses, without delays for recharging of capacitors. The rise time from 0 to $100 \mathrm{~V}$ was less than $1 \mathrm{~ms}$, fast enough compared to typical action potential durations on the order of $100 \mathrm{~ms}$. One practical requirement was that the whole setup be mobile in order to quickly set up the experiment in the operation room and move all devices out again after finishing the experiment. We mounted the amplifiers onto a standard wheeled 19 " rack (figure 6.2). In the course of the experiments, it turned out that the required power was so low that the amplifier system could be reduced to just the two digital $1 \mathrm{~kW}$ devices (1) and (2)).

\subsubsection{A Custom-Built Defibrillator}

The peak power which could be delivered by the power amplifier assembly described in the last section was sufficient for the open-chest experiments with patch electrodes. For the experiments with catheter shock electrodes and ICD case however, larger energies per pulse were necessary.

To fulfill these increased requirements across one or many user-defined shocks, we had a custom experimental defibrillator built at and in collaboration with the electronics workshop at the Third Institute of Physics at the University of Göttingen. According to 


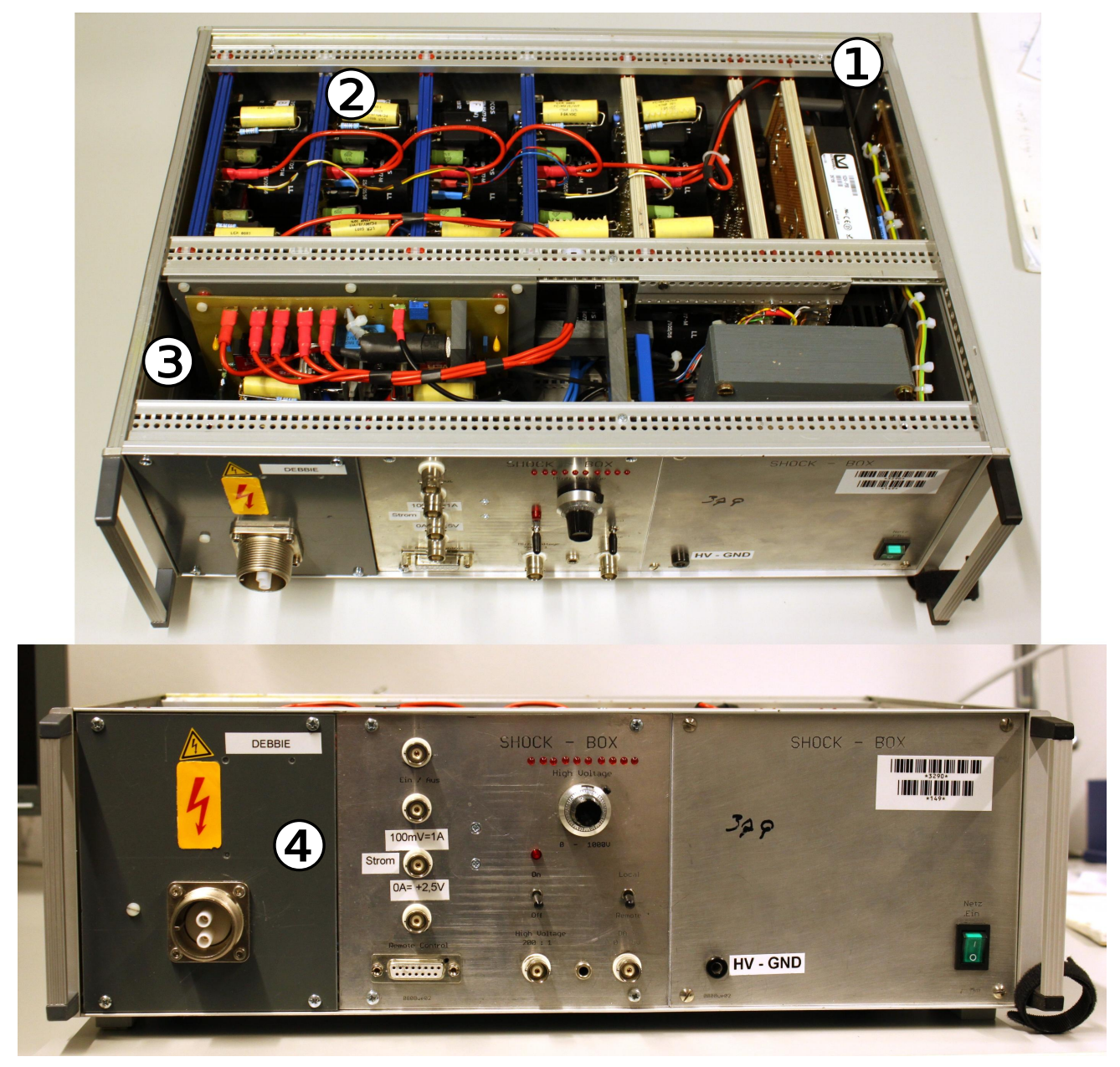

Figure 6.3: The experimental LEAP defibrillator. Top view of the internal boards: (1) The high voltage transformer used to charge the capacitors and the control logic board. (2) Five capacitor banks for up to 10 high voltage pulses. (3) The bridge board to route the capacitors to the experiment connector and to switch polarity if required. (4) Front panel with high voltage connector (left), and control/sensing links and charge display (middle panel)

our specifications regarding voltage, energy and number of pulses, Dr. Lautscham and Mr. Ebrecht at the workshop developed a LEAP prototype defibrillator.

This defibrillator can deliver up to ten monophasic shocks with $1000 \mathrm{~V}$ peak voltage and $78 \mathrm{~J}$ per pulse, corresponding to five symmetric biphasic shocks of over $150 \mathrm{~J}$ each.

In order to be able to deliver the required high peak power, our defibrillator is based on the same principle as commercial defibrillators, using charged capacitors. Here, the defibrillator (see figure 6.3 for pictures) contains ten capacitor banks of $155 \mu \mathrm{F}$ which are charged through a high voltage power supply (1C24-P30, Ultravolt, USA). Charging takes approximately $40 \mathrm{~s}$ from a fully discharged state to the maximum of $1000 \mathrm{~V}$. The maximum voltage can be set either manually on the front panel or remotely via an analog voltage input (stepped up by a factor of 200). Each capacitor bank (see figure 6.3 for an interior view) can be discharged separately through a digital input to the defibrillator, 


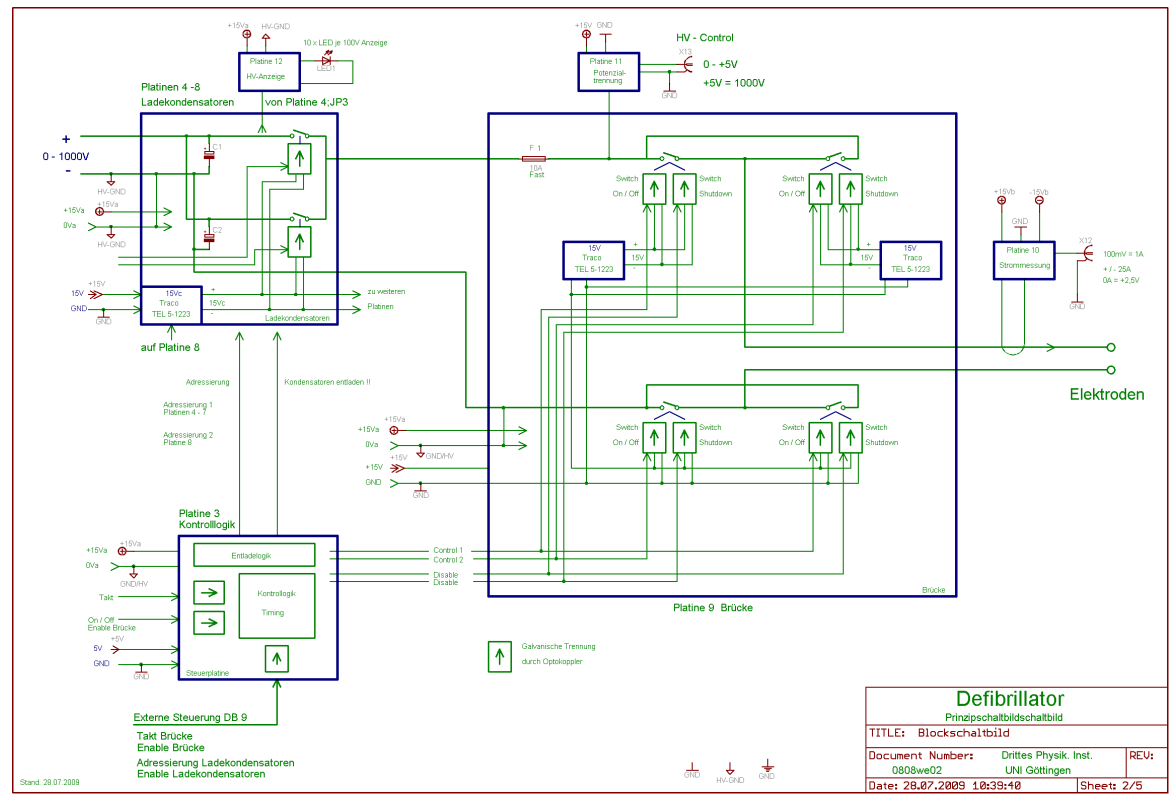

FIguRE 6.4: Overview schematic diagram of the experimental LEAP defibrillator, showing control of the output bridge and capacitor charge/discharge control. Schematic by Ebrecht/Lautscham

the polarity can be chosen as required and switched during or between pulses. For safety reasons, the capacitors automatically discharge upon shut-down or power loss.

The defibrillator was accessed by a DA/AD converter (NI USB-6259 BNC, National Instruments, USA) which was controlled by a PC running self-written LabView (National Instruments, USA) control software, similar to the power amplifier described in the previous section. This software allowed us to monitor one arbitrarily chosen bioelectric signal, for example the ECG and, if deemed necessary, to automatically calculate the temporal defibrillation parameters from its time series.

At the same time, data from (electro)physiological sensors and the defibrillator current and voltage was recorded for simultaneous and later analysis (figure 6.5). Using for example the peak values of current and voltage of the last shocks to calculate the impedance, our software can autonomously choose the correct voltage for a desired energy.

In the example of figure 6.5, the impedance was $50 \Omega$, the peak current at the initial voltage of $399 \mathrm{~V}$ was $8 \mathrm{~A}$. With less than one quarter of its maximum power, the custom-built defibrillator successfully defibrillated the heart (pig, 2011-12-12) where the combination of four power amplifiers would have failed. As can be seen in the plot, about two seconds after the shock, the blood shows a significant level of oxygen again. 


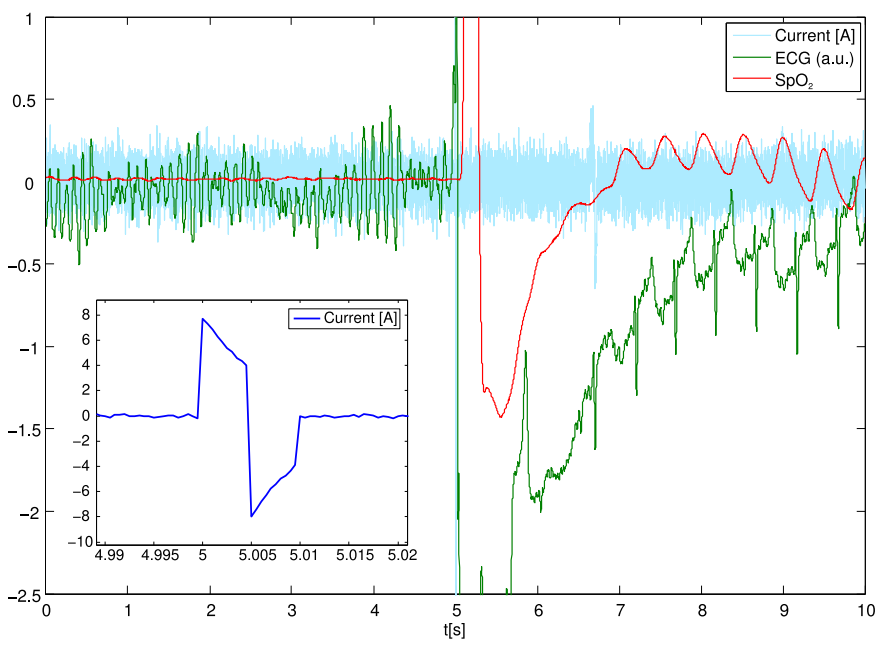

Figure 6.5: Time series of a successful single pulse defibrillation. Shown are the defibrillator current (light blue, dark blue in the inset), one ECG lead, and the blood oxygen saturation. The inset shows the exponentially decaying current during the two phases of the shock.

\subsubsection{Electrical Connection to the Heart}

\subsubsection{A Temporarily Implanted Defibrillator}

To be as close to clinical application as possible, we used commercially available ICD catheters (Sprint Quattro, Medtronic, USA) which were inserted into the right ventricle (RV) of the animal. The electrically relevant part consists of a shocking coil and two electrodes for recording and low-energy stimulation, see also figure 6.6a.

Figure 6.6b shows the other electrode, a stainless steel replacement for the ICD can which was embedded under the left pectoralis major muscle. This guaranteed with little effort that the electric field would be as similar to the one in humans with ICDs as possible. Both parts, the catheter and the ICD phantom were safely connected to the defibrillator devices, if necessary, the connection could be switched to a backup defibrillator within a few seconds.

\subsubsection{Epicardial Mesh Electrodes}

Similarly to the experiments in [45], we decided to use mesh electrodes on the epicardium for the open chest dog experiments. Additionally to better comparability to previous studies, we expected advantages with respect to the field geometry and absolute energy requirements. We used flexible wire mesh (stainless steel) patches of $10 \mathrm{~cm}^{2} \times 10 \mathrm{~cm}^{2}$ to which we welded solderable contacts of about 3 to $4 \mathrm{~cm}$ width (see figure 6.7 and the electrodes in use in figure 6.11). In-situ, the mesh was cut with common scissors so as to fit to the heart's shape. 


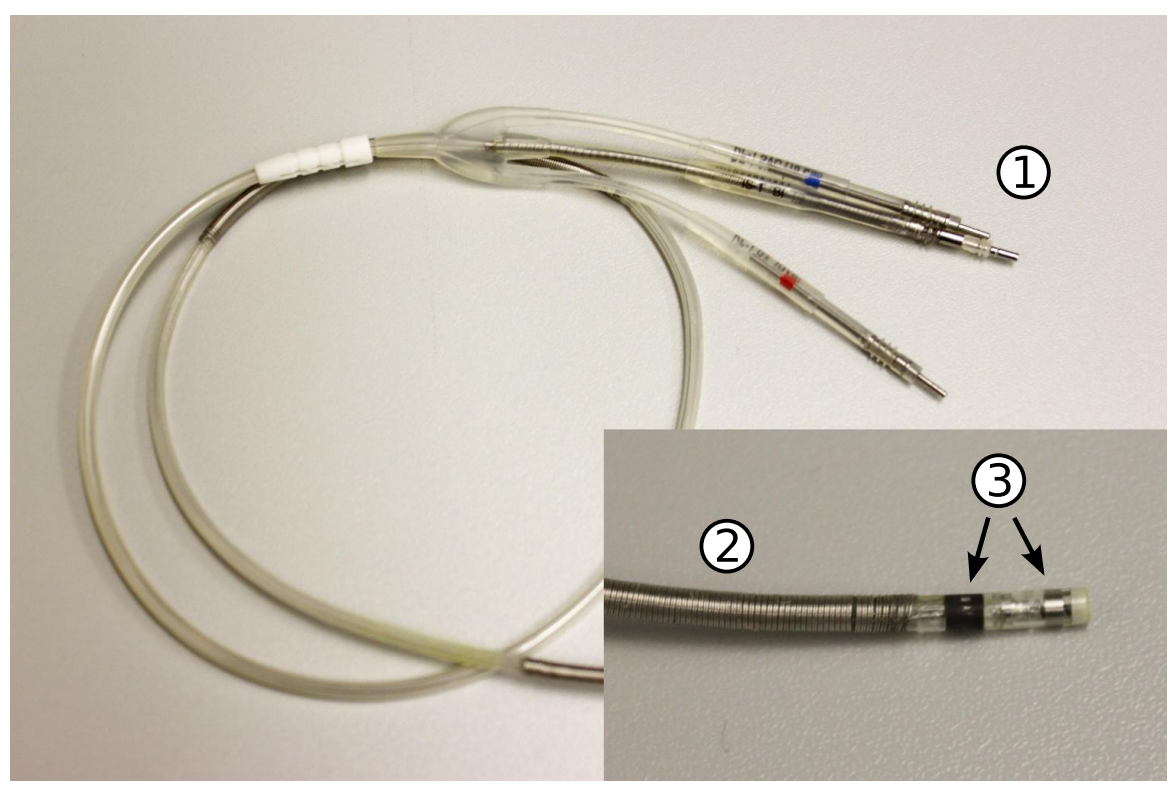

(a) An ICD catheter of the type used in the in vivo experiments. The recording and defibrillation equipment is connected at (1). Note the two small electrodes at the tip of the catheter (3), the corkscrew emerges from the tip and fastens the catheter tip into the tissue. The coil (2) serves as a high surface area electrode during defibrillation shocks.
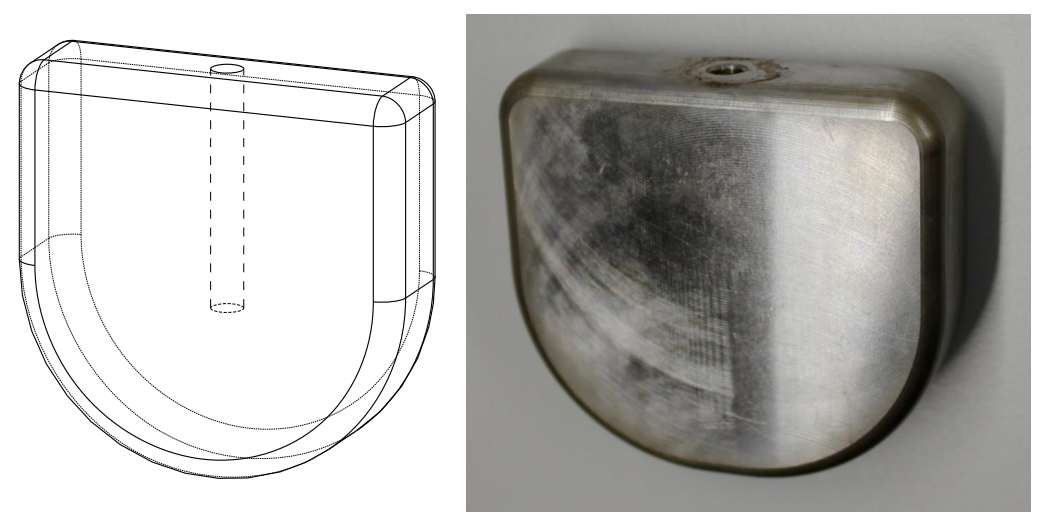

(b) The ICD phantom used in the ICD experiments. Its size and shape $(55 \mathrm{~mm} \times 50 \mathrm{~mm} \times 15 \mathrm{~mm})$ correspond to ICD cans that are routinely implanted to human patients.

FiguRE 6.6

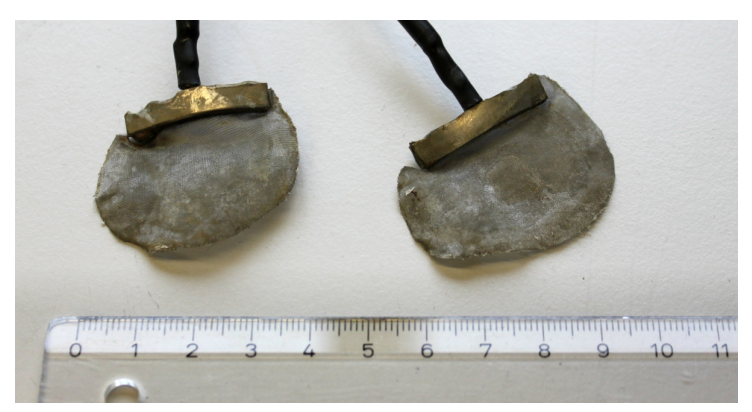

Figure 6.7: Mesh electrodes used in the open chest experiments. The shape was individually adapted by cutting the mesh with normal scissors. 


\subsubsection{Physiological Measurement Recording}

During the experiment, we recorded data from electrophysiological and other medically relevant sources, for two-fold purposes: It enabled us to adapt the experimental settings in the course of the experiment and allows for detailed analysis of possible dynamical effect of success or failure of defibrillation afterwards.

All signals were captured at $2 \mathrm{kHz}$ with commercial hardware modules (Biopac, USA) and recorded with the software package Acqknowledge (Biopac, USA). In all experiments, we recorded the following signals:

- Three-lead ECG, taken from the upper right and left chest and near the left hip joint. Due to the different orientation of the heart in quadrupeds (the apex is oriented towards the sternum), the recorded signal differs from the well-known ECG in humans.

- Action-potential recordings from the tip of the intraventricular catheter. The potential difference across the two small electrodes on the catheter (see figure 6.6a) gives a reliable signal about the electrical activity in the vicinity of the catheter. This is also the configuration which is used by commercial ICDs to monitor and record the electrical activity in the heart.

The action potential and ECG signals were amplified and directly fed into the Biopac AD converter. To prevent potential risks for users and the hardware modules during high voltage events, the leads were fitted with an overvoltage protection.

- Blood oxygen saturation $\left(\mathrm{SpO}_{2}\right)$ and exhalation $\mathrm{CO}_{2}$ concentration were measured at the tongue or ear and the respiration apparatus, respectively. During fibrillation, these easily accessible vital parameters give an indication how ischemic the organism is.

- Current through and voltage across the defibrillator output, whether it be our custom-built prototype or the high power amplifiers. During defibrillation attempts, only the shock voltage is set, while the impedance of the system is generally unknown. To obtain the delivered energy, the exact values of current and voltage over time are necessary.

During the experiment, we used the electrophysiological recordings (ECG and local action potential at the catheter on the endocardium) to estimate the current dominant frequency of the fibrillation and to decide the pacing frequency of the next defibrillation attempt (see figure 6.8 for a screenshot of the acquisition software). 
The time-resolved time series of current and, depending on the configuration, voltage, allowed to calculate the delivered energy per pulse. Generally we calculated the energy by first computing the setup's impedance $R$ as the ratio of maximum voltage and current. With this information, the energy $E_{\Omega}$ for a pulse could be calculated by

$$
E_{\Omega}=\int_{\Omega} R \cdot I(t)^{2} \mathrm{~d} t .
$$

\subsubsection{The Animal Operation Room Setup}

The operation room setup was equivalent for the in vivo experiments for pigs and dogs. The setup was designed to provide all necessary means required by animal welfare guidelines and for a maximum efficiency in the experiment. The animals were held in anesthesia by artificial respiration with pure $\mathrm{O}_{2}$ supplemented with $1-2 \%$ isoflurane. The artificial respiration guaranteed a minimum of ischemia during the periods of reduced or suppressed cardiac pumping. The recorded signals mentioned in the previous section were continuously monitored.

In addition to our experimental defibrillator / power amplifiers, we had a commercial defibrillator implant support device (Medtronic DISD 5358, Medtronic, USA) available in standby as a backup defibrillator system to minimize the fibrillatory period. As a secondary backup, an external defibrillator with paddles was available in the OR for cases when the experimental defibrillator or the first backup failed, e.g. because the catheter was in an unfavorable position.

For the pig in vivo experiments, we had a mobile fluoroscopic X-ray system available to control the correct positioning of the ICD catheter inside the heart. This was not necessary for the dog open chest experiments, since there the catheter's position could be seen directly.

\subsection{Experimental Procedure}

For the in vivo pig LEAP experiments, we used $N=9$ juvenile male domestic pigs (Sus scrofa domesticus, German Landrace) and $N=5$ adult male Göttingen Minipigs. We chose the pigs' ages so that irrespective of their race, they weighed approximately $30 \mathrm{~kg}$ (mean \pm s.d. $35 \pm 11 \mathrm{~kg}$ ). The beagle dogs were about 14 months (mean \pm s.d. $14.3 \pm 1.7$ months) old and weighed about $10 \mathrm{~kg}$ (mean \pm s.d. $9.7 \pm 1.3 \mathrm{~kg}$ ). All animal handling and 


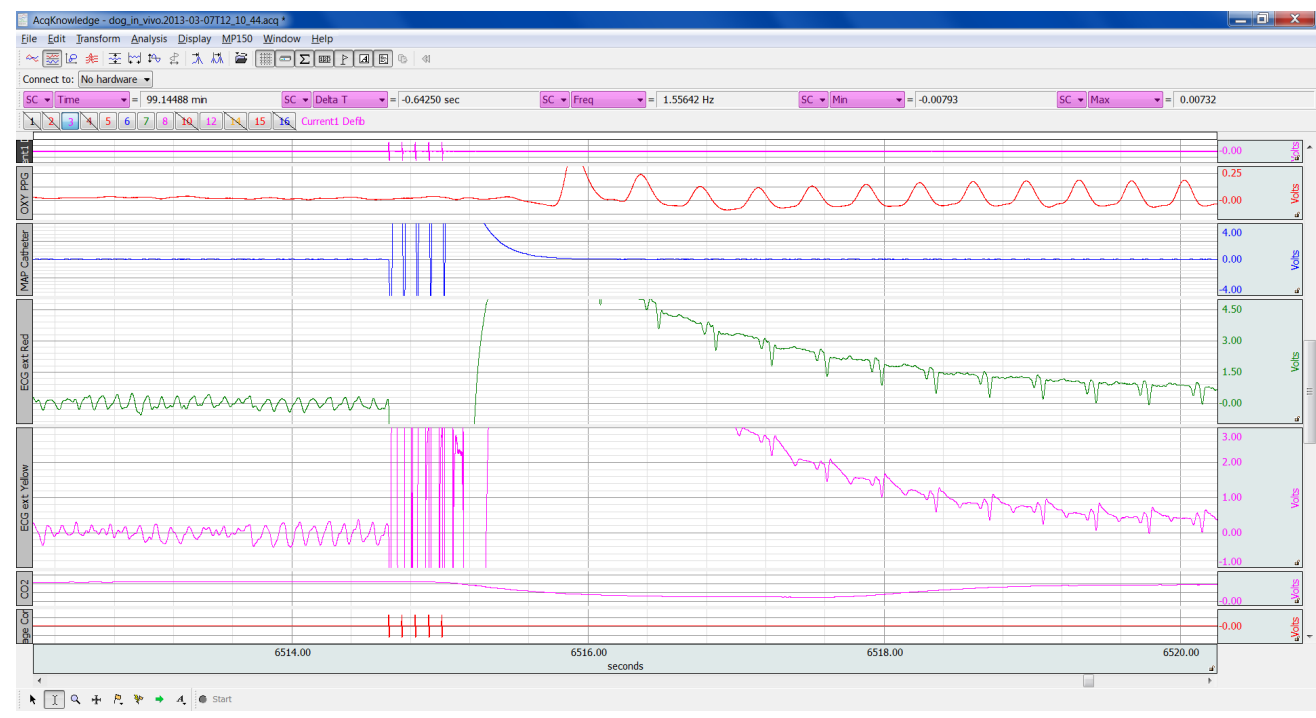

(a) Data acquisition software

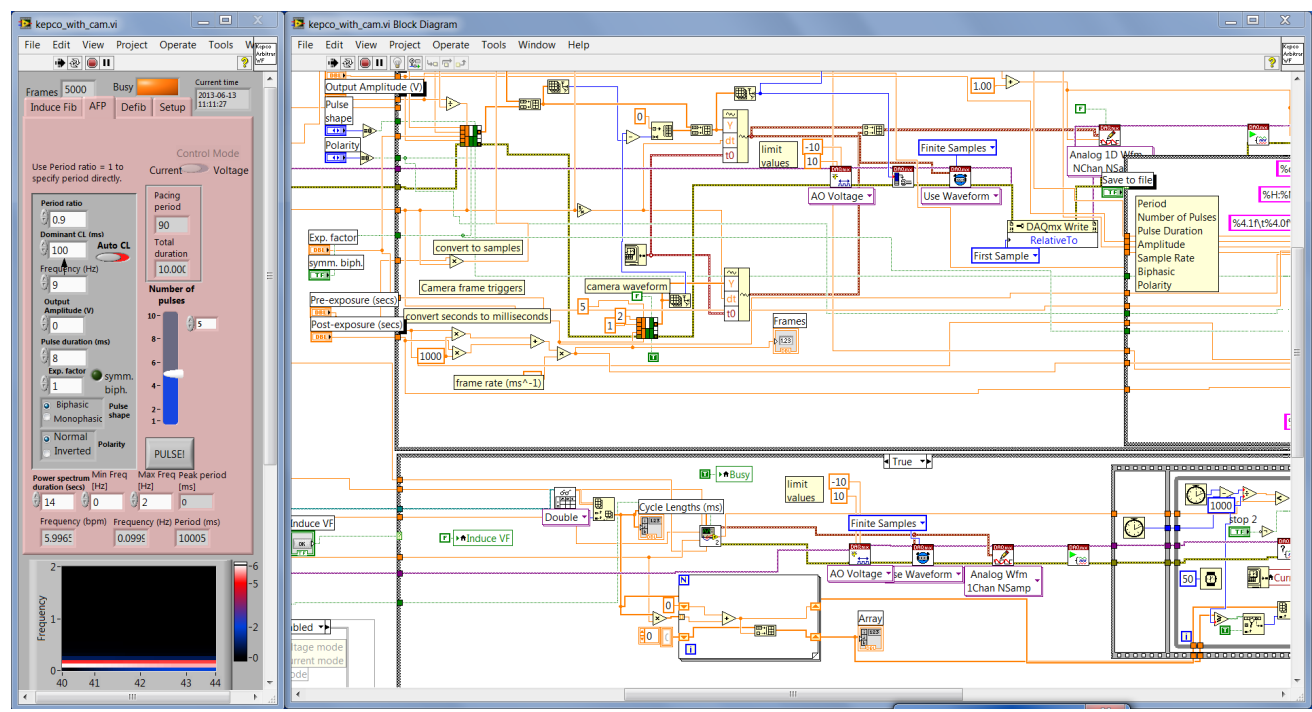

(b) Defibrillator control software

FIgURE 6.8: Screenshots of the software used for data acquisition (Acqknowledge, Biopac, USA) and defibrillator control. (a) The relevant recorded time series during defibrillation with LEAP. 5 pulses terminate the fibrillation with $99 \mathrm{~V}$, single pulses with $130 \mathrm{~V}$ failed to defibrillate before and after this event. The two ECG traces (green and magenta) and the blood oxygen saturation (red) indicate successful defibrillation. (b) The control software front panel (left) and part of its internal structure (right), written in LabView (National Instruments, USA). With this custom-written software, we could control the number of pulses, voltage, pacing period, polarity, pulse duration and pulse shape and switch between mono- and biphasic pulses.

the experimental procedures were approved by the responsible authorities (the Niedersächsisches Landesamt für Verbraucherschutz und Lebensmittelsicherheit (LAVES) for the pig experiments and the Institutional Animal Care and Use Committee (IACUC) at Cornell University for the dogs) and experienced veterinarians were present during the experiments to supervise the procedures and to ensure the animal was free of pain at all 


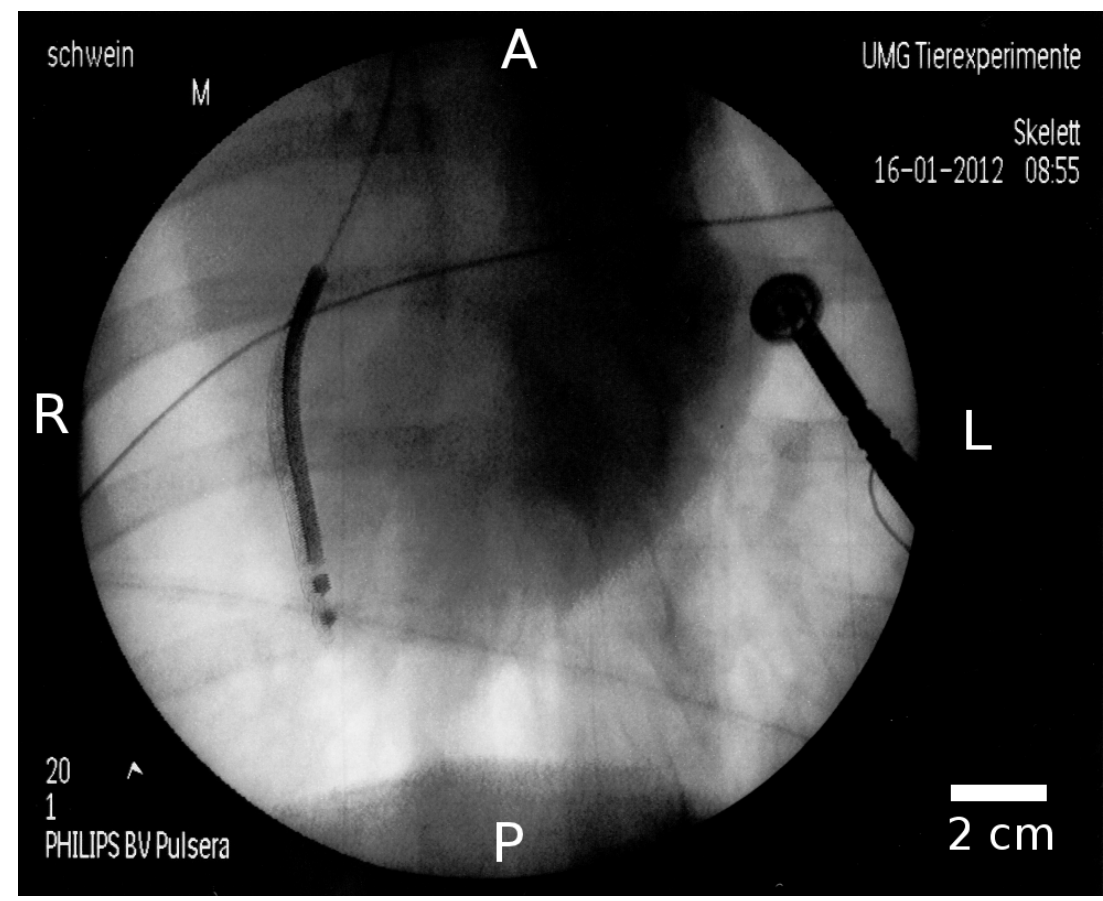

Figure 6.9: Fluoroscopic ventral view of an ICD catheter in the RV of a pig heart. Right, left, anterior and posterior directions are marked on the picture. Note the two sensing/stimulating electrodes at the tip of the catheter. The slight double image of the catheter coil is due to the heart's beating during exposure.

times.

\subsubsection{Pre-Fibrillation}

The animals were anesthetized with fentanyl and sedated with propofol by the veterinarians, intubated and connected to the respirator $\left(100 \% \mathrm{O}_{2}\right.$ with $1-2 \%$ isoflurane). Capillary $\mathrm{SpO}_{2}$, the exhaled $\mathrm{CO}_{2}$ concentration and the electrocardiogram were continually monitored. During the whole experiment, the body temperature was kept constant at a physiological level with blankets or electric heating.

\subsubsection{ICD Phantom Implant}

In the pigs, an ICD catheter (two sensing/stimulating electrodes at the tip, 1-2 coils for delivering defibrillation shocks, Medtronic, USA) was inserted via the jugular vein into the right ventricle (RV). Under fluoroscopic positioning control, its tip was secured at the endocardium close to the apex by means of a corkscrew-type fastener embedded into the catheter. After this procedure, the coil was completely inside the RV. For an example of the final catheter position, comparable to human ICD implants, see the printout of a fluoroscopic image in figure 6.9 . 


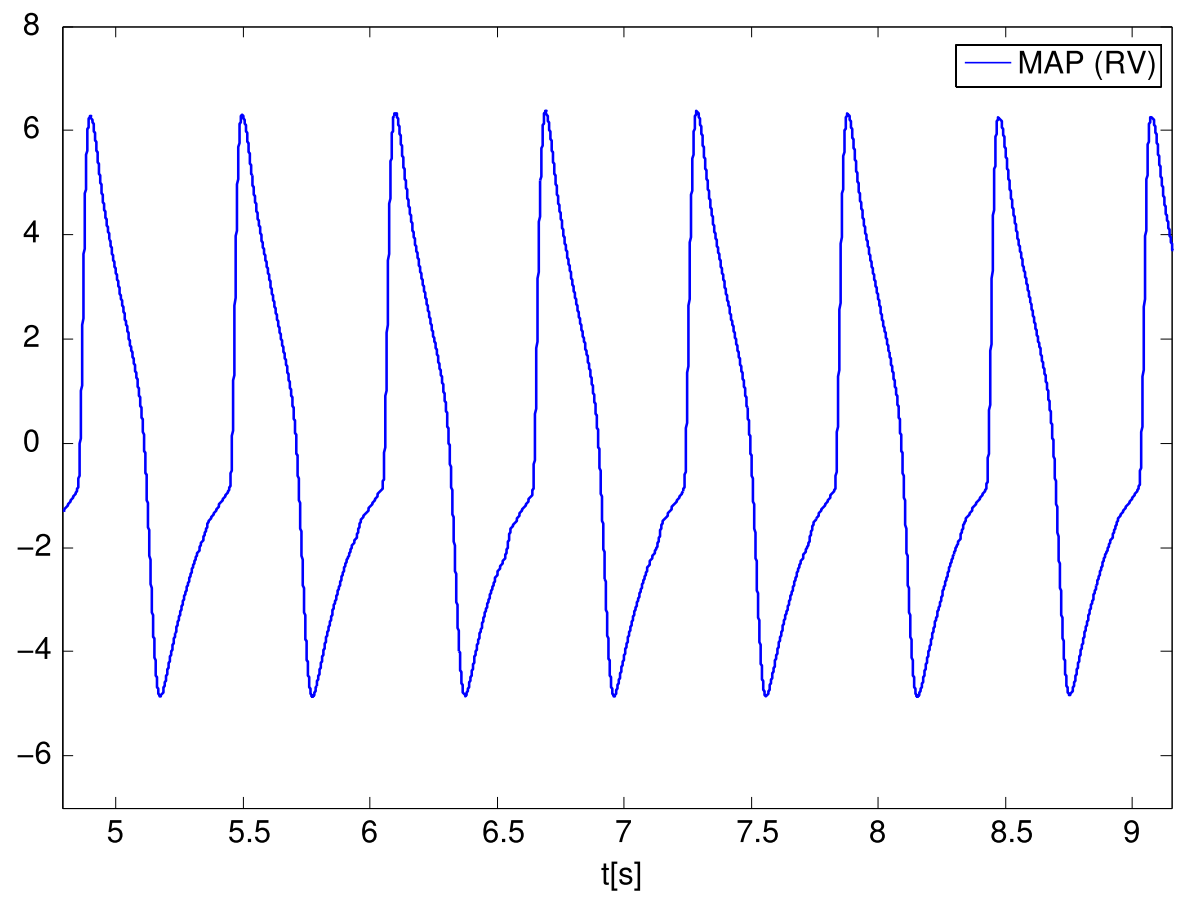

FIgURE 6.10: RV monophasic action potential (MAP) as measured with an ICD catheter electrode in a pig heart.

Subsequently the ICD case phantom (figure 6.6b) was placed into a pocket under the pectoralis major m., about $15 \mathrm{~cm}$ from the catheter coil. The catheter and the case were connected to the defibrillator sets and to the monitoring/stimulating modules.

The correct position of the catheter tip was verified by two independent means: the electric signal should show an undistorted action potential (figure 6.10) and a low pacing threshold for the stimulating electrodes (around $1 \mathrm{~V}$ at $0.5 \mathrm{~ms}$ pulse duration) was verified (with a Medtronic Analyzer 2290, Medtronic, USA).

\subsubsection{Open Chest Electrode Application}

In the open chest dog experiments, after induction of anesthesia, the thorax was opened up via sternotomy. The pericardium was incised and epicardial electrodes were attached to the epicardium. To keep the impedance between the defibrillators and the heart and the electric field inside the heart as constant as possible in the course of an experiment, we secured the patch mesh electrodes to the epicardium and filled any remaining gap with conductive gel. In the course of the experiment, the mesh and gel were kept wet by adding saline as necessary and before each fibrillation episode. The patches were fixed to the epicardium either with several sutures or by applying a few drops of commercially available superglue (figure 6.11). 


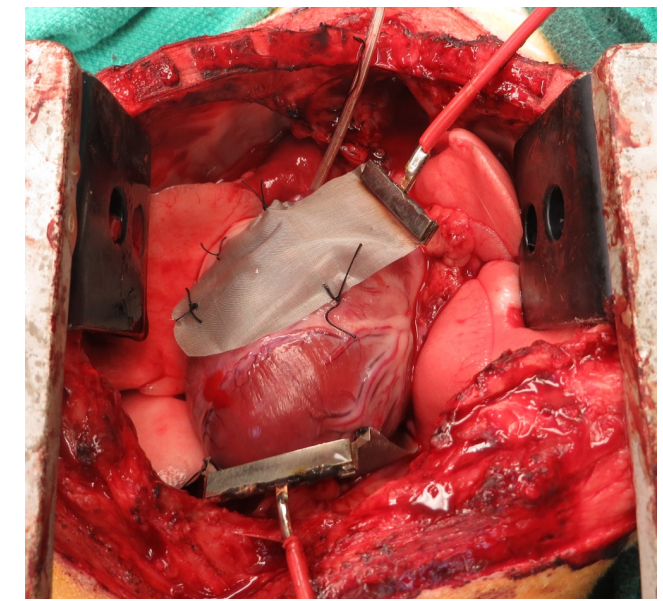

(a)

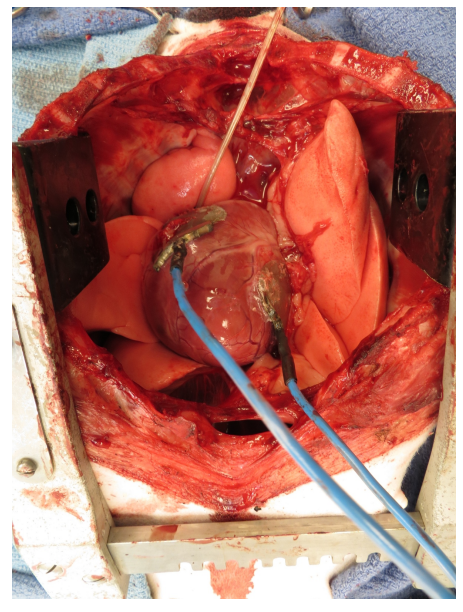

(b)

FIgURE 6.11: Patch mesh electrode in situ secured onto a dog heart in open chest LEAP experiments. The electrodes in (a) were sutured to the epicardium, the electrodes in (b) were held in place with common superglue. The orientation of these images is the same as in figure 6.9

In order to find the configuration with the lowest energy requirements, we used several distinct electrode configurations: with an additional ICD catheter electrode inside the RV and without. We also varied the size of the patches from covering nearly the whole heart to pieces of about $3 \mathrm{~cm}$ diameter.

In all experiments, the ICD catheter was inserted through the opened thorax and the v. cava superior into the RV.

\subsubsection{Initial Impedance Measurement}

After completing the setup of the high voltage electrodes, the shock impedance was measured with the implant support device by delivering a $0.2 \mathrm{~J}$ test pulse. In case this pulse would induce an arrhythmia in the heart (it was not synchronized with the ECG), the same Medtronic device could be used to immediately defibrillate if necessary. This impedance then served as a scaling factor during the experiment to estimate the shock energy from the applied voltage and to set the voltage so that a desired energy was reached.

The measured impedances with catheter-can configurations were in the same range as for human ICD implants (20 to $100 \Omega$ [100]); for epicardial patch electrodes, they were significantly higher, probably due to the fact that since at least one electrode was in the air, no current could flow around the heart: 


\begin{tabular}{r|r|r|r} 
Electrode setting & $N$ & Impedance mean & s.d. \\
\hline catheter-can (pig) & 14 & $65.8 \Omega$ & $7.2 \Omega$ \\
epicardial patch (dog) & 6 & $92.8 \Omega$ & $14.2 \Omega$
\end{tabular}

\subsubsection{Defibrillation to Evaluate LEAP Efficiency}

The main purpose of the series of experiments was to assess the efficiency of LEAP compared to single pulse defibrillation, measured as the energy per pulse required to successfully terminate VF.

The general procedure for all (de)fibrillation episodes was: induction of VF, waiting $10 \mathrm{~s}$ to verify that the VF would not terminate itself, termination of VF within less than 1 min after induction, letting the heart and animal recover before the next episode.

We induced fibrillation by stimulating the heart near the apex with the tip of the ICD catheter for about $3 \mathrm{~s}$ with $50 \mathrm{~Hz}$ and just sufficient voltage to obtain capture (as could be verified in the ECG traces). Subsequently we waited for $10-15 \mathrm{~s}$ to ensure that the fibrillation was stable.

No more than 20-30s after effective pumping of the heart stopped (which means that the VF induction time had to be included), we attempted to terminate the fibrillation, either with a single defibrillation shock in order to determine the defibrillation threshold (DFT) or with LEAP (5 pulses of lower energy), varying the energy, wave form or the pacing frequency's ratio to the fibrillation's dominant frequency.

If this attempt failed, we immediately switched the wiring to connect to the commercial backup defibrillation device and defibrillated the heart with a single biphasic shock of $34 \mathrm{~J}$ (for the catheter-can configuration in the pigs) or $10 \mathrm{~J}$ (for the patch electrode setup in the dogs). In nearly all cases of primary failure to defibrillate, the first backup shock was sufficient to terminate the fibrillation at once. In those cases where the backup shock failed as well, the external defibrillator was loaded and prepared for discharge. If during this preparation time further backup shocks by the primary backup defibrillator did not terminate the arrhythmia, the external defibrillator was discharged as many times as necessary to get the heart out of fibrillation.

If the heart stayed in asystole for more than a few seconds after fibrillation, we stimulated it with low voltages at physiological frequencies (about 1 to $2 \mathrm{~Hz}$ depending on the species) until normal sinus rhythm set in again.

After each such episode of fibrillation and termination, we waited several minutes to let the animal recover from the period of ischemia. This waiting time differed depending 


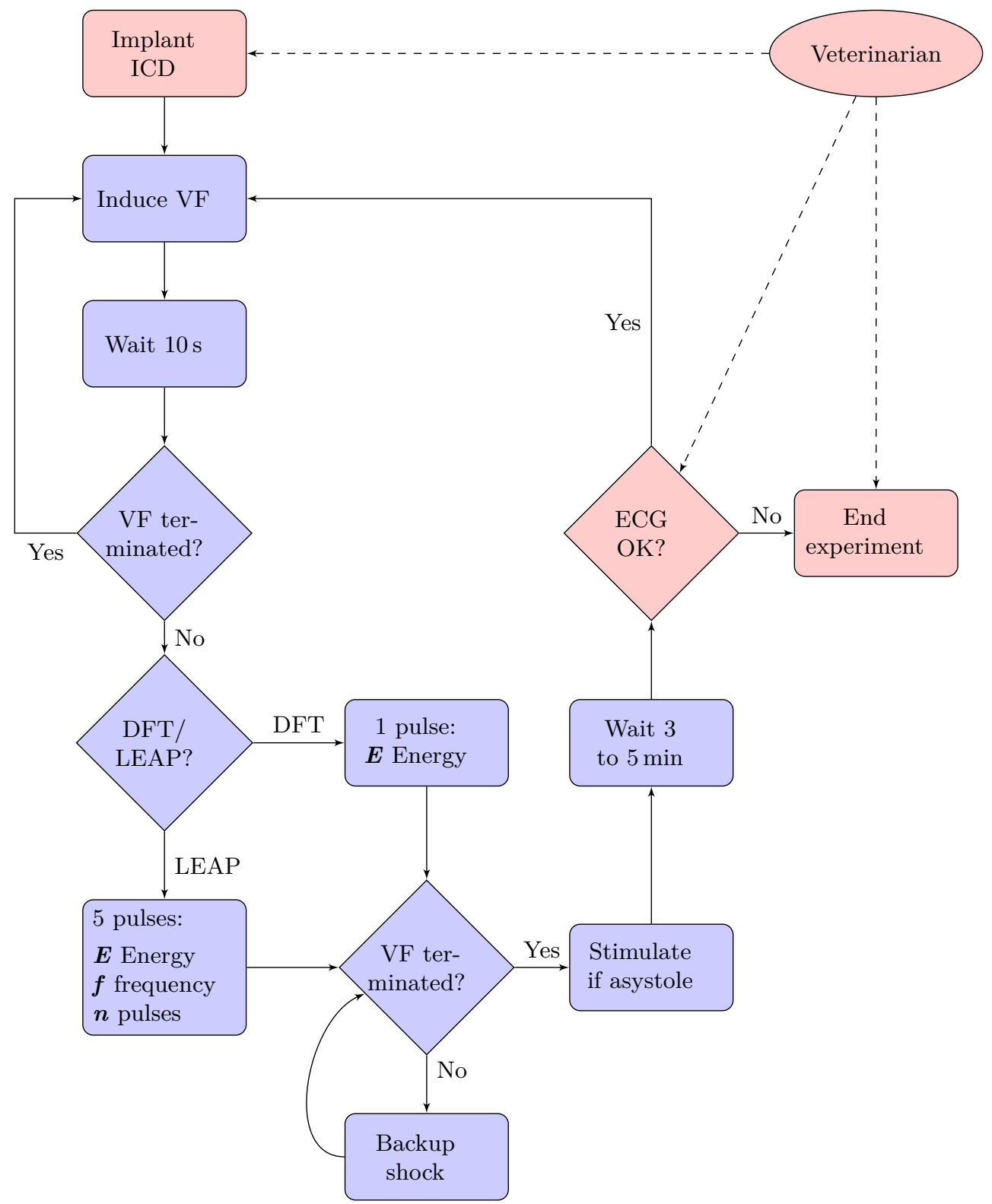

FiguRE 6.12: Schematic of the in vivo experimental protocol.

on the ECG interpretation of experienced cardiologists who were present during the experiments, it was mostly about 2 to 3 min long. In a few experiments we deliberately waited considerably longer ( 7 to 10 minutes) to check if the recovery time had any influence on the DFT.

\subsubsection{Choosing the Defibrillation Parameters}

Our aim was to measure the possible energy gain through LEAP, this gain is always relative to the energy required for defibrillation by single shocks. Unfortunately there is no sharp cut-off energy above which defibrillation suddenly happens, but the DFT can 
only be defined in terms of defibrillation success probabilities. To detect possible drifts in the DFT, we measured it first at the beginning of an experiment and later at least once every hour. For this we attempted to defibrillate with a single shock at a supposedly above-threshold energy. If the defibrillation attempt was successful we decreased the energy by some amount (usually $2 \mathrm{~J}$ ), otherwise we increased it by the same amount. This up-down procedure was repeated until the "success barrier" was crossed at least two times, i.e. until we had gone from successful to unsuccessful and back again or vice versa.

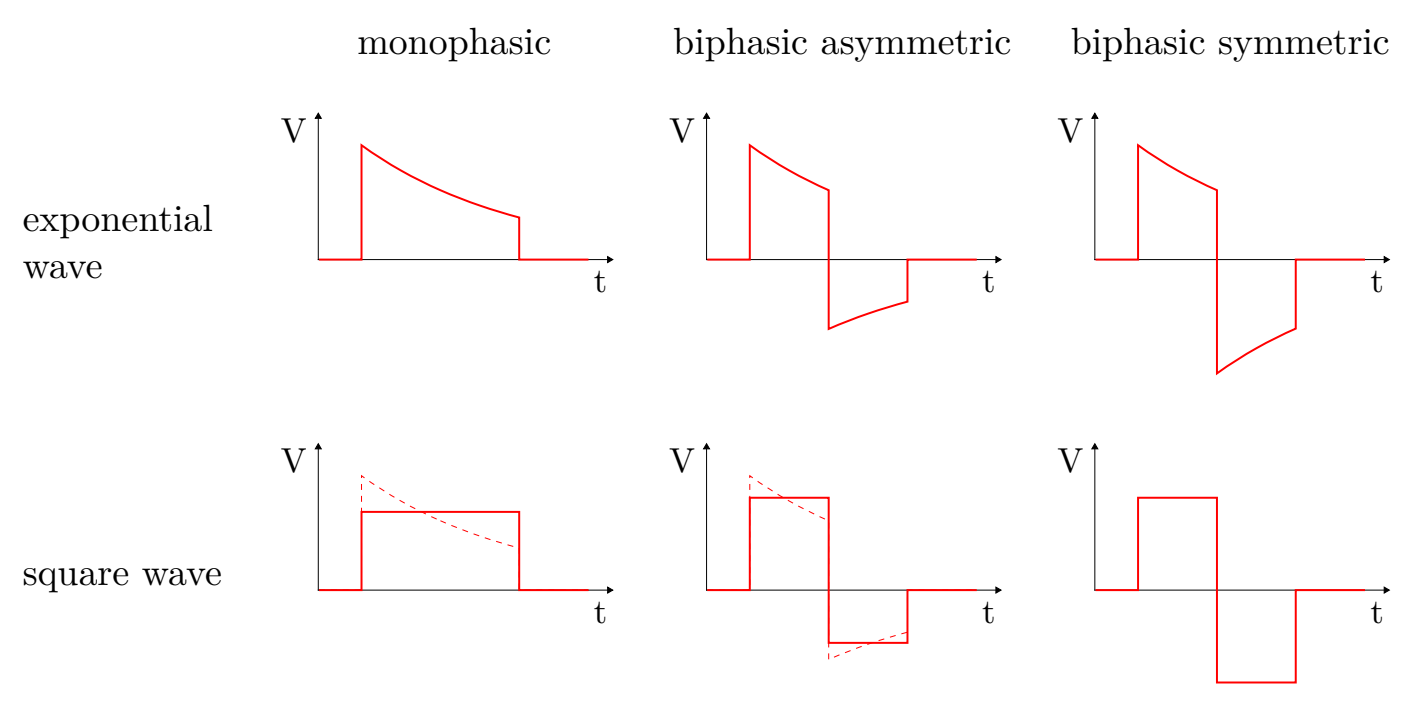

TABLE 6.1: Overview over defibrillation shock wave forms.

Additionally to the energy, the wave form also plays a role in determining defibrillation success, table 6.1 shows an overview over some characteristics of shock pulses. The following are the parameters we had to choose, some of which only apply to LEAP pulses, though:

Single Pulse Shape Clinical defibrillators, implanted and external, use capacitors to deliver a large amount of energy in a short time, just as the custom-built defibrillator we used for the pig experiments. In contrast in the dog experiments, we were able to define arbitrary wave forms, especially square pulses instead of exponentially decaying voltages as in discharging capacitors (first row of table 6.1). We mostly chose square pulses (second row) because ex vivo experiments showed no difference between the DFT for both wave forms and with square pulses the same energy could be delivered with less peak power. 
Mono-/biphasic Instead of discharging the capacitor at once (monophasic pulse, first column of pictures in table 6.1), it is possible to switch polarity during the discharging process (biphasic pulse, second column). Since it is widely known that biphasic shocks have higher probabilities of terminating fibrillation than monophasic shocks of the same energy [101, we used only biphasic shocks in nearly all experiments.

Wave Symmetry When a single capacitor is used to produce a biphasic pulse, the second part naturally has lower voltages (second column). We decided mostly on either discharging another capacitor or setting the voltage symmetrically in the power amplifiers (third column) in order to deliver a maximum of energy and to be more independent of the configuration's impedance, which influences the steepness of the exponential decay and thus the voltage and power ratios of the two pulse parts.

Frequency Ratio When pacing with more than one pulse, the temporal coordination with the fibrillation pattern becomes important. For termination of tachycardia (ventricular and atrial), local pacing slightly faster than the dominant cycle length (antitachycardia pacing, ATP) [102, 103] is the preferred method of driving the heart into a healthy rhythm again. For termination of VF with LEAP which uses far-field pacing, previous studies have been inconclusive [45] if higher or lower frequencies compared to fibrillation obtain the best energy gains. Therefore we investigated broad frequency ranges, from about 0.7 to 1.3 times the dominant frequency.

Number of Pulses Theory and numerical simulations predict [47, 48, that for LEAP to succeed in unpinning a pinned spiral wave, the phase of the pulse relative to the spiral must fall into the unpinning window. Several pulses increase the chance that one of the pulses falls into the unpinning window and when $n_{\text {pulse }}=f_{\Omega \Omega} /\left(f_{\Omega \Omega}-f_{\Im}\right)$, the whole phase should be covered (with $n_{\text {pulse }}$ being the number of pulses and $f_{\Omega \text { 几 }}$ and $f_{\circledast}$ being the frequencies of the pacing and the pinned spiral, respectively). This reasoning is not perfect though, since effects like phase resetting as described in [49] may arise. With frequency differences of up to approximately $20 \%$, we stayed with $n_{\text {pulse }}=5$ for nearly all experiments, this was also the maximum our custom-built defibrillator could deliver for biphasic symmetric pulses. We did not gather meaningful amounts of data for $n_{\text {pulse }}$ other than 1 or 5 .

\subsubsection{End of LEAP Experiment}

For all in vivo specimen, we continued the experiment as long as technically and ethically possible. We stopped to do further defibrillation attempts, if the heart showed signs of 


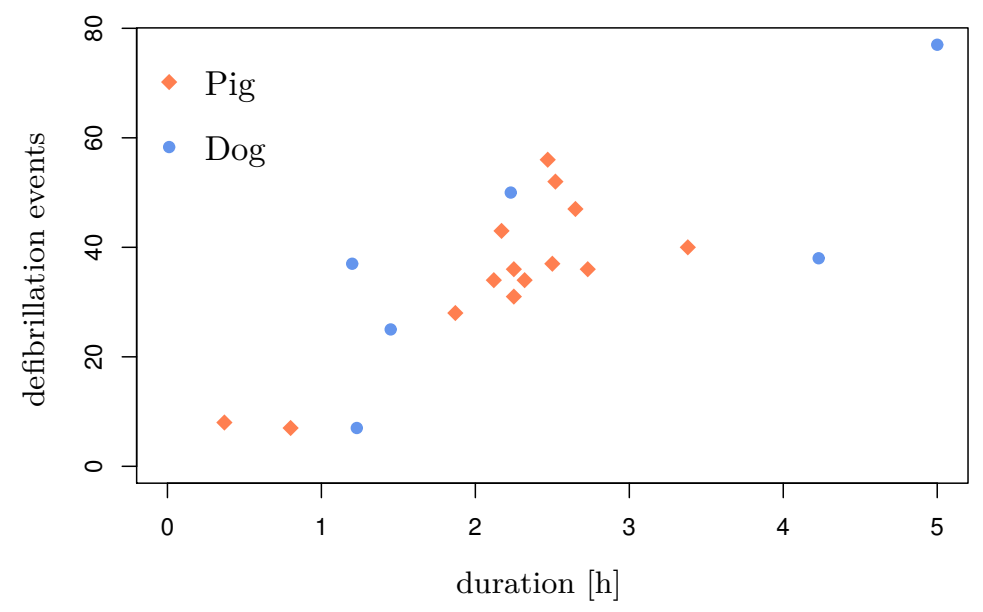

Figure 6.13: Experiment durations and number of defibrillation events for the in vivo experiments. Times measured from the first to the last defibrillation episode. The two short pig experiments (bottom left corner) were one where defibrillation failed early in the experiment (leftmost) and where the catheter detached from the endocardium (second from the left).

substantial change in behavior, such as:

- much slower recovery after fibrillation events

- difficulties to terminate VF (increased DFT)

- VF would terminate spontaneously or after very low shocks (decreased DFT)

- after professional advice from the veterinarians, e.g. after drop of blood pressure

Usually the experiments lasted for over 2 hours (average for pig experiments: $2.2 \mathrm{~h}$, dog experiments: $2.6 \mathrm{~h}$ ), the maximum was $3.4 \mathrm{~h}$ (pigs) and $5 \mathrm{~h}$ (dogs), respectively. In this period, we were able to perform on average 35 and 39 defibrillation attempts (see also figure 6.13.

After the decision to end the in vivo experiment, the animal was prepared for the following Langendorff perfusion. We administered sufficient heparin to prevent post-mortem blood coagulation $(400-500 \mathrm{IU} / \mathrm{kg})$ a few minutes before the veterinarians applied a lethal dose of pentobarbital. After the animal's death, while still under artifical respiration, we flushed the heart with approximately $500 \mathrm{ml}$ of ice cold cardioplegic solution (high-K, approximately $8 \mathrm{mmol}$, exact composition in section 7.4.1) in order to arrest the heart in the diastolic state and to preserve it as well as possible for the following $e x$ vivo experiments.

Subsequently the heart was extracted, with median sternotomy if necessary, submerged into $0{ }^{\circ} \mathrm{C}$ cardioplegia solution, with any air removed from the vasculature and stored until the beginning of the ex vivo experiments (see section 7.4). 



\section{Chapter 7}

\section{Ex Vivo LEAP Experiments}

In addition to the in vivo experiments described in the previous chapter, we conducted two series of ex vivo low energy antifibrillation pacing (LEAP) experiments with pig and dog hearts. Similar experiments have previously been performed on smaller samples, either in intact rabbit hearts under Langendorff perfusion [94, 104] or in wedge preparations of larger hearts (dog hearts, [45]). We continued in this direction in order to fill the gap between the more controlled environments of in vitro and the more clinically relevant in vivo experiments.

\subsection{Benefits of Ex Vivo Antifibrillation Experiments}

Although in vivo studies have advantages such as a naturally realistic physiological embedding and the same configurations of electrical field and current as in clinical applications, there are a number of undisputed benefits of ex vivo defibrillation experiments. Besides the ethical and economical advantage of obtaining as much knowledge as possible from a heart after an in vivo experiment, there are the following points:

\subsubsection{A Controlled Environment}

In contrast to experiments in living animals, the electric field can be defined comparatively freely. The freedom of choosing the electrode configuration allows to study how the field geometry influences the energy requirements of single pulse defibrillation and LEAP. Whereas electrode configurations with one intraventricular catheter coil and an ICD always lead to regions with no or at least negligible fields (according to Walgott et al. [36], the field on the far side of the heart is only $1 / 10$ as large as on the side 
facing the ICD can), in ex vivo setups the $E$ field can be chosen to be nearly homogeneous. Conversely, it is of course also possible to reproduce the $E$ field of in vivo configurations or to adapt the electrode configuration in order to optimize upcoming in vivo experiments.

Furthermore, the controlled environment of a perfusion setup minimizes changes in the experiment over time such as movement of the heart relative to the electrodes and keeps chemical and physiological changes at a minimum. The continuous perfusion with oxygenated solutions such as Krebs-Henseleit solution or Tyrode's solution ensures a steady supply of oxygen to the tissue, thereby removing the risk of ischemia inherent to in vivo antifibrillation experiments. Possible adverse effects of long-term fibrillation itself can not be excluded in this way, but they could be differentiated from effects of ischemia.

\subsubsection{Experimental Efficiency}

Without ischemic periods, it is not necessary in perfusion experiments to give backup shocks should the fibrillation not be terminated immediately after induction. Combined with the absence of recovery periods between fibrillation episodes, this leads to a much higher efficiency in terms of defibrillation attempts per time. Whereas in vivo, one defibrillation attempt required about 4 minutes, in the perfusion setup, the average time between two attempts was as low as 30 seconds. The defibrillation results show no difference regarding the long-term behavior of defibrillation energy compared to the in vivo experiments (chapter 8), so it is a valid assumption that in spite of the different time scales the results are comparable. For an overview over the ex vivo number of defibrillation attempts also compare figure 7.6 to figure 6.13 .

\subsubsection{Contactless Activation Measurement}

Major advantages of a perfusion setup are the accessibility of the whole surface of the heart for experimental measures and the independence of the heart on its pumping function. With voltage sensitive dyes, the cells' activation can be measured with high spatial resolution and without electrical contact. This circumvents the problem that measuring the low amplitude electrophysiological signals directly is not possible during shocks. Also the recording amplifiers usually need several seconds after the delivery of a defibrillation shock to recalibrate themselves to the normal signal level again. Optical mapping of cardiac excitation does not suffer from these limitations and thus can give reliable information about success or failure of antifibrillation pacing. 
Additionally the spatial resolution of optical mapping allows more complex analyses of the heart's spatio-temporal behavior, e.g. through activation maps under different electric fields.

\subsection{Open Questions}

With the ex vivo experiments, we tried to gain insight into the following problems:

\subsubsection{Comparability with In Vivo Experiments}

Any results in abstracted setups have to be gauged against how well they can be applied to "reality", in our case clinically relevant configurations. Since the most realistic setup available at present are in vivo experiments, the question arises if results from in vivo and ex vivo experiments are comparable. Therefore we checked, whenever possible, for differences between the outcome of both experiment types.

\subsubsection{Comparison to other LEAP Evaluations}

Theory [47] and previous experiments [45] predicted and showed high energy gains of LEAP compared to single pulse defibrillation. Since the ex vivo experiments performed so far were only done on smaller hearts or on heart preparations, an open question remains whether these results can be transferred to larger systems. We reproduced the experimental setup of previous studies in large parts so that differing outcomes would probably be due to reasons inherent to the samples.

\subsubsection{Frequency Dependency of LEAP Success}

There is no clear consensus yet at which frequencies LEAP works best. Since it takes several defibrillation attempts to measure the efficacy of a given defibrillation parameter set, scanning the pacing frequency at several energies is only feasible in setups where many defibrillation episodes can take place in a short time. Perfusion systems have a clear advantage compared to a in vivo setup here (see also 7.1.2. 


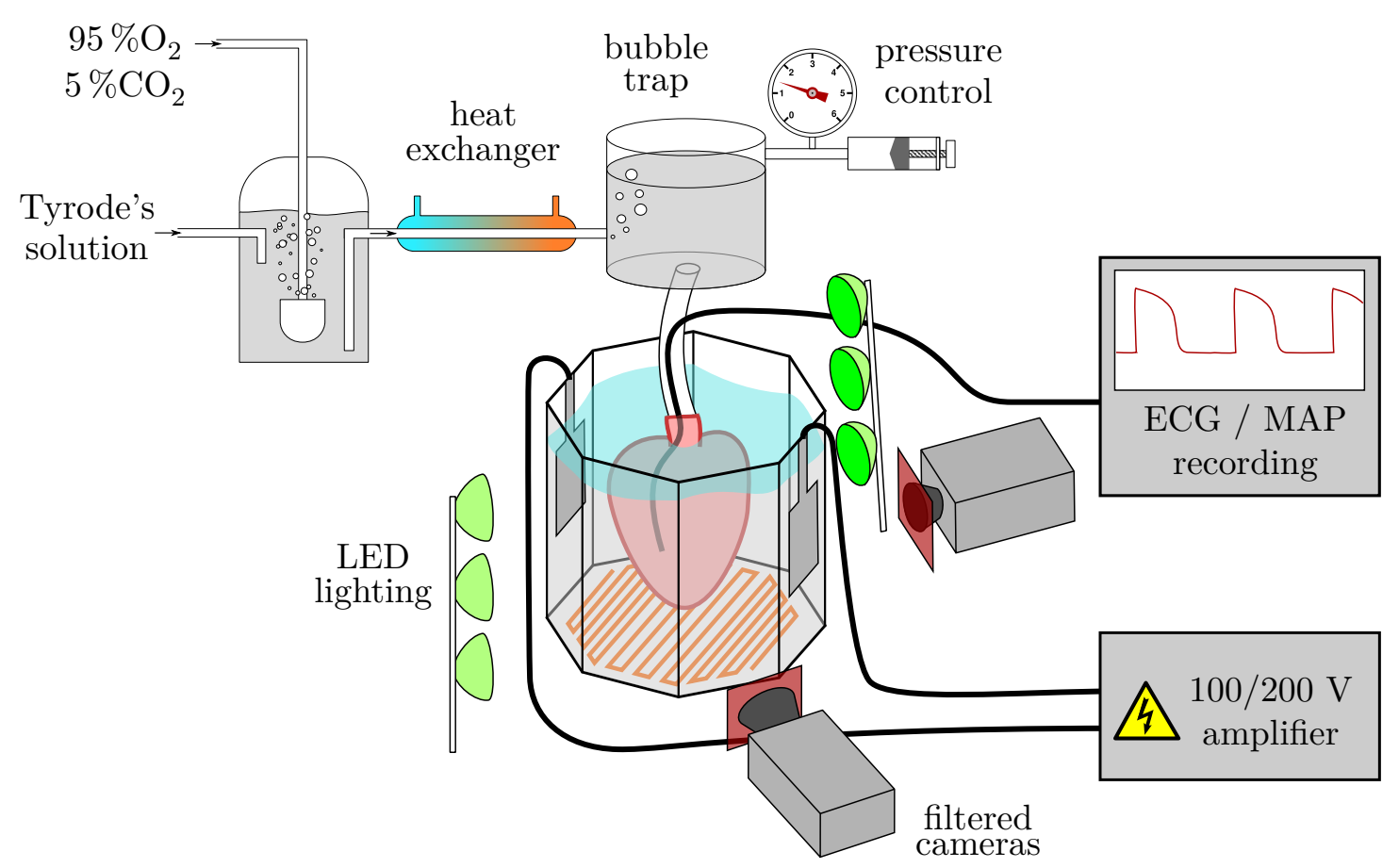

FIGURE 7.1: Outline schematic of the perfusion experiment setup. With graphics from http://www.openclipart.org

\subsection{The Experimental Setup}

We set up the ex vivo experiments based on previously described configurations concerning the perfusion, the optical mapping of electrical activity and electric field geometry [94, 105.

Figure 7.1 shows an overview over our experimental setup. We perfused the isolated heart with a warm physiological buffer solution in a transparent glass bath. The bath was fitted with electrodes for measuring the ECG and with antifibrillation pacing panels. The heart was stained with a voltage sensitive dye, illuminated at the excitation wavelength and cameras recorded the optical activation signal through emission wavelength filters. Further details about the setup are given in the following sections.

\subsubsection{The Langendorff Perfusion System}

Like any organ, the heart needs a sufficient supply of oxygen, nutrients and a physiological level of ion concentrations (most prominently $\mathrm{Na}^{+}, \mathrm{Ca}_{2}^{+}, \mathrm{K}^{+}$) in order to maintain all necessary physiological functions. We perfused the heart with a Krebs-Henseleit/Tyrode solution bubbled with carbogen $\left(95 \% \mathrm{O}_{2}\right.$ and $\left.5 \% \mathrm{CO}_{2}\right)$. The heart itself was suspended less than $10 \mathrm{~cm}$ from a bubble trap and pressure regulation block (Harvard Apparatus, USA) in the Krebs-Henseleit/Tyrode solution bath. The bath was kept at a constant 


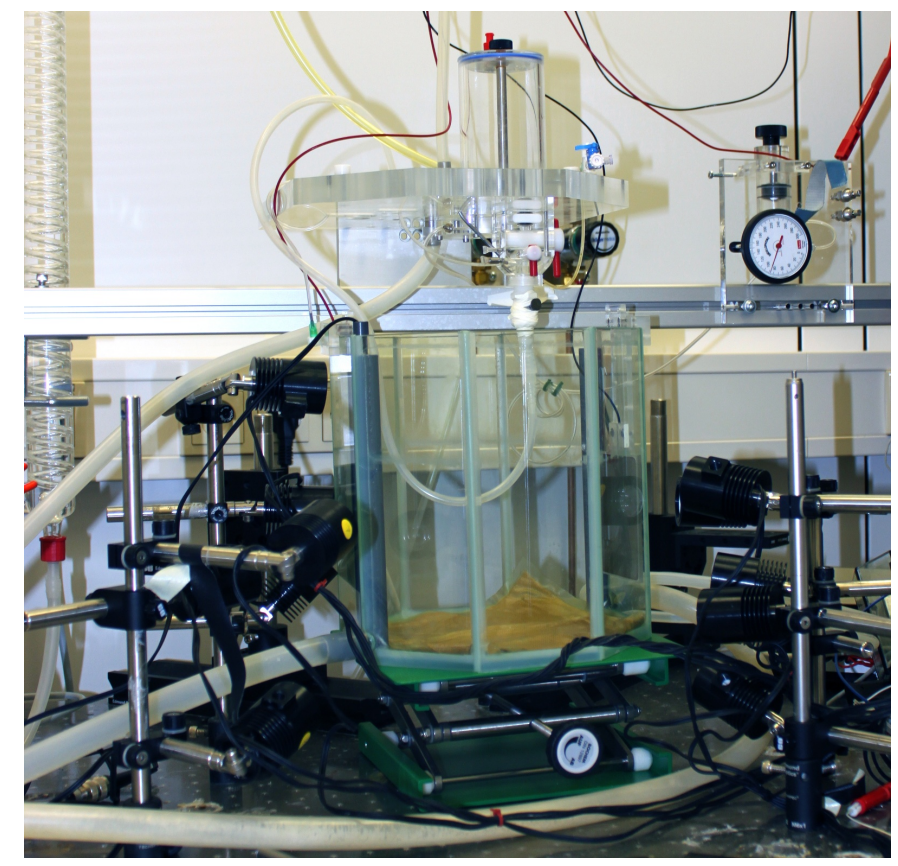

Figure 7.2: The general setup of the ex vivo perfusion experiment. Centered: the glass bath in which the heart is suspended during the experiment. Above: The aortic block, consisting mainly of a cylindrical bubble trap (cf. figure 7.1) which also reduces pressure variation from the pump (not on the image) and a pressure regulator (to the right). The LEDs around the bath excite the fluorescent voltage sensitive dye.

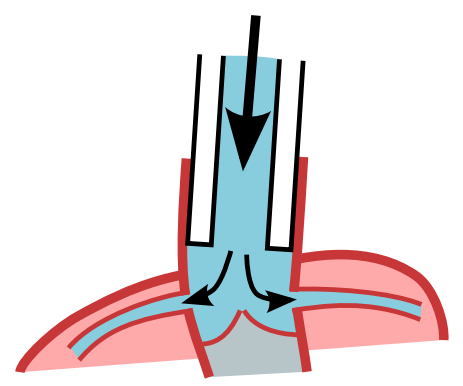

FiguRE 7.3: The principle of Langendorff perfusion: The perfusion is connected to the aorta, its pressure closes the aortic valve (bottom) to the left ventricle (gray area). Therefore, the perfusion solution flows completely into the coronary arteries, keeping the cardiac muscle alive.

temperature of 36 to $37^{\circ} \mathrm{C}$ (slightly below the physiological temperature of 37 to $39^{\circ} \mathrm{C}$ [106]), by actively compensating heat losses of the bath and by pre-heating the perfusate. We adjusted the perfusion pressure to approximately $100 \mathrm{mmHg}(13 \mathrm{kPa}$ [106], a physiological value for awake and anesthetized swine) at flow rates of approximately $300 \frac{\mathrm{ml}}{\min }$.

In the Langendorff perfusion, which we used for all whole-heart experiments, the perfusing system is connected retrogradely to the aorta (figure 7.3 ). Since the aortic valve is still functional, the perfusate flows mainly into the coronary arteries and supplies the cardiac muscle with oxygen. The perfusion system also served as a pathway for administering drugs to the heart as necessary. 


\subsubsection{Optical Mapping}

As described in more detail above (section 4.6.1.1), we stained the hearts with 1 to $2 \mathrm{ml}(10 \mu \mathrm{M})$ of voltage sensitive dye di-4-ANEPPS ${ }^{1}$. In order to obtain a sufficiently high optical intensity, we illuminated the heart with 12 (for the pig heart) or 20 (dog) high power green LEDs (pig: $3 \mathrm{~W}$, dog: up to $5 \mathrm{~W}$ per LED, peak wavelength $530 \mathrm{~nm}$, LUXEON $^{2}$ ). The pig hearts were suspended in an octagonal custom-built glass water bath with $23 \mathrm{~cm}$ diameter. Through up to four sides of this bath, we recorded the electrical activity with electron multiplication CCD (EMCCD) cameras (CASCADE $128+{ }^{3}$ ) at native frame rates of up to $500 \mathrm{~Hz}$. The cameras were fitted with long pass filters (cutoff edge at $590 \mathrm{~nm})^{4}$ to maximize the intensity changes of the stained tissue. During the experiment, the heart was immobilized by the excitation-contraction decoupler blebbistatin to prevent motion artifacts in the signal traces of single camera pixels.

The cameras were controlled and the images were recorded with a custom-written program, MultiRecorder ${ }^{5}$. Additionally to recording the movies, this program allowed to show the image signal and time traces from selected image regions. This gave us the possibility to monitor in near real-time if the defibrillation was successful and if pacing had any effect at all. Furthermore the power spectrum of the trace(s) was automatically calculated, showing the dominant fibrillation frequency which was then used by us to calculate the LEAP frequency.

\subsubsection{The Electrical Configuration}

During the ex vivo experiments, we used a monophasic action-potential (MAP) catheter inside the RV to record an action potential signal from the RV apical region. This catheter could also be used for stimulation of the heart and, in those cases where it was the ICD catheter which was used in vivo previously, as a shock electrode.

Additionally, we placed two stainless steel needle electrodes on the sides of the bath. Being only influenced by the heart activation's electrical far field, their potential difference was equivalent to classical ECG signals, which provided us with an alternative way to determine the fibrillation frequency.

The configuration of the shock electrodes was chosen in each experiment according to the particular heart preparation:

\footnotetext{
${ }^{1}$ Life Technologies, Darmstadt, Germany

${ }^{2}$ Philips Lumileds, San Jose, CA, USA

${ }^{3}$ Photometrics, Tucson, AZ, USA

${ }^{4}$ Edmund Optics, Karlsruhe, Germany

${ }^{5}$ Main author: Johannes Schröder-Schetelig
} 


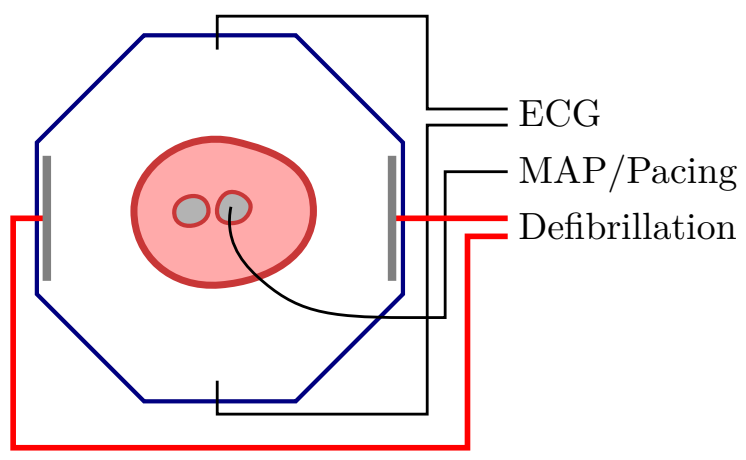

Figure 7.4: Top view of the perfusion bath in the pig setup. Two stainless steel plates serve as defibrillation shock electrodes (left and right). Two needle electrodes, arranged perpendicularly in order to minimize the voltage at the amplification module, sense the heart's electric far-field in the manner of ECG electrodes. This electric sensing is supported by an MAP electrode in the right ventricle which also works as a stimulation pacing site.

Wedge Preparations For the wedge preparations (the first three pig heart experiments), we separated the left ventricle including the interventricular septum (201202-14 and 2012-05-07) or the right ventricle (2012-06-11) from the rest of the heart. In the case of LV preparations, we cut along the septum from the base to the apex to place the ventricle in a flat manner into the bath, similar to the individual RV preparation. The preparations were perfused through tubing leading directly into the main arteries. For the wedge preparations we did not use the octagonal bath but a flat container with heatable double walls. On two opposite sides of the tissue, we set up small $\left(2 \mathrm{~cm}^{2} \times 4 \mathrm{~cm}^{2}\right)$ mesh electrodes for far-field pacing.

Intact Pig Heart The shock electrodes for the whole pig heart consisted of $3 \mathrm{~cm} \times 4 \mathrm{~cm}$ stainless steel plates fixed at opposite sides of the water bath (figure 7.4).

Intact Dog Heart For the dog hearts, we generally used the same electrode configuration as during the in vivo experiment before, i.e. patch electrodes on the epicardium.

In all cases, the shock electrodes were connected via a safety switch to the defibrillator devices we used, either one or more power amplifiers or the custom-built experimental capacitor-discharging defibrillator.

We used the same LabView software as in vivo for controlling the defibrillator devices. Likewise the electrical signals were amplified and digitized with Biopac modules and recorded with the Acqknowledge recording software. The defibrillation control software also synchronized all up to four cameras with the defibrillation attempt to enable later analysis of the videos. 
FiguRE 7.5: Impedances of in vivo and ex vivo (only whole heart) experiments. The impedance means are pairwise significantly different between all setups $(p<0.01)$ except between the ex vivo values for pigs and dogs, respectively. The low ex vivo impedances meant that high currents were required in order to deliver the necessary defibrillation energy to the heart.

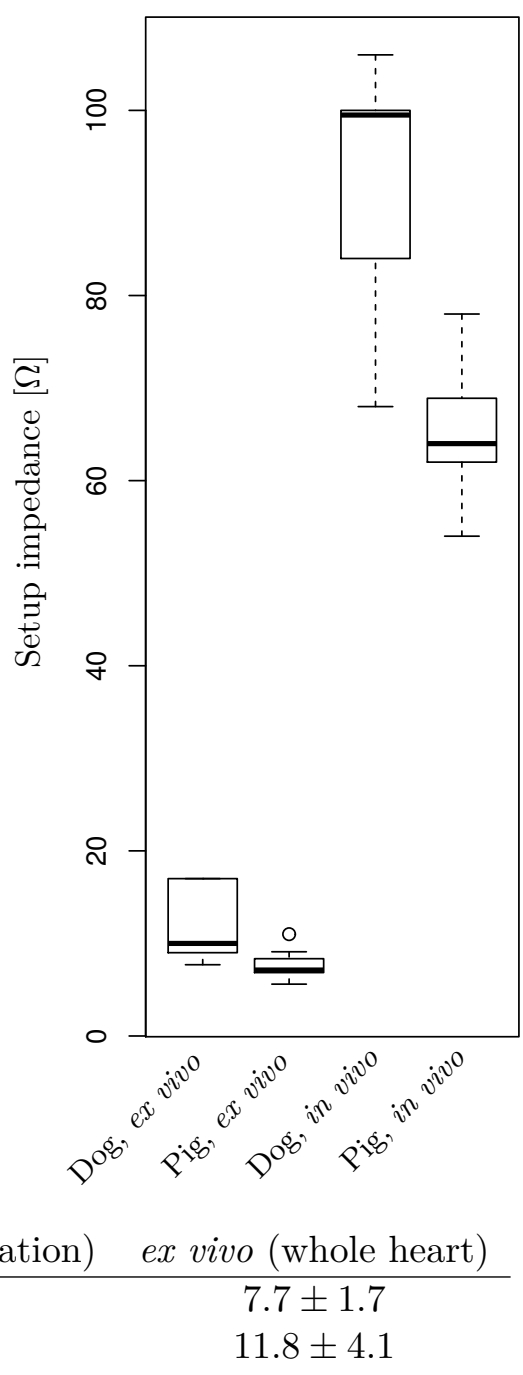

TABLE 7.1: In vivo and ex vivo impedances compared. Impedance (mean \pm standard deviation) given in $\Omega$.

\subsubsection{Effective Energy Transfer}

Maybe the most important physical difference between the in vivo and ex vivo setup is not the heart itself but its environment. In contrast to the complex mixture of bone, muscles, air (in the lungs) and other tissue, the specific conductivity of the perfusion solution in the bath is comparatively low. The impedance for all in vivo experiments ranged from 54 to $106 \Omega$, whereas the ex vivo experiments (intact heart only) had impedances in the range of 5.6 to $17 \Omega$ (see table 7.1 and figure 7.5 for mean values and standard deviation).

Especially in the pig heart experiments, it remains an open question how much of the current actually flows through the heart instead of around it and through the Tyrode solution. The heart's cross-sectional area was about one third as large as the bath's area and the cardiac muscle conductivity (about $0.5 \frac{\mathrm{S}}{\mathrm{m}}$ and even lower if transverse to the 
fiber orientation [107]) is much lower than the conductivity of an approximately isotonic saline solution $\left(1.4 \frac{\mathrm{S}}{\mathrm{m}}[107]\right)$. This meant that, as opposed to the setup in the in vivo experiments, most of the current would not flow through the heart, which increases the total power necessary for defibrillation. The electrical configuration posed no problem for the dog heart experiments where we had the mesh electrodes placed directly on the heart's surface. In this setuup, more of the delivered energy was able to activate the cardiac tissue and thus terminate fibrillation.

In the pig heart experiments, we performed some defibrillation attempts with our capacitor discharging experimental defibrillator. Two times during these experiments, the capacitor current was too large (at or above $60 \mathrm{~A}$ ) and some electronic parts were damaged, although lower voltages or currents failed to defibrillate the heart. When using the power amplifiers, we sometimes had to increase the duration of the pulses considerably in order to defibrillate with single shocks.

\subsection{Experimental Procedure}

We performed successful ex vivo LEAP experiments on 11 pig and 6 dog hearts. All hearts were excised from animals after they were sacrificed after the end of in vivo experiments which were performed according to the local animal welfare regulations (approvals by Niedersächsisches Landesamt für Verbraucherschutz und Lebensmittelsicherheit (LAVES) for the pig experiments and Institutional Animal Care and Use Committee (IACUC) at Cornell University for the dogs). Most of these preceding experiments were LEAP experiments we conducted ourselves (see the previous chapter for more information), and some pigs were used before in non-cardiac related experiments [108] of the OMS department ${ }^{6}$ at the University Medical Center Göttingen. The following table gives an overview over the number and origins of the examined animal hearts for all successful ex vivo experiments.

\begin{tabular}{lll} 
animal & origin & number of hearts \\
\hline Pig (German Landrace, juvenile) & LEAP experiment & 1 \\
& LEAP experiment & 2 \\
Pig (Göttingen Minipig, adult) & OMS experiment & 8 \\
Dog (Beagle) & LEAP experiment & 6
\end{tabular}

\footnotetext{
${ }^{6}$ Oral and maxillofacial surgery (Abteilung Mund-, Kiefer- und Gesichtschirurgie an der Universitätsmedizin Göttingen), the experiments tested tooth implants.
} 


\subsubsection{Sample Preparation}

Prior to excision of the heart, the animal was given heparin and cardioplegic (with a high $\mathrm{Ka}^{+}$concentration) solution to prevent blood coagulation, to arrest the heart in the diastolic state and to protect it during the following period of reduced metabolism [109]. Immediately after the heart was taken out of the chest, it was put into a container with more ice-cold cardioplegia solution until further preparation.

The cardioplegia solution had the following molar concentrations:

\begin{tabular}{llr} 
& \multicolumn{2}{l}{ Cardioplegia solution } \\
& $\mathrm{NaCl}$ & $110 \mathrm{~mm}$ \\
& $\mathrm{KCl}$ & $16 \mathrm{mM}$ \\
& $\mathrm{MgCl}_{2}$ & $16 \mathrm{~mm}$ \\
& $\mathrm{CaCl}_{2}$ & $1.2 \mathrm{mM}$ \\
TABLE 7.2: Composition of the cardioplegic & $\mathrm{NaHCO}_{3}$ & $10 \mathrm{mM}$ \\
& $\mathrm{MgCl}_{2}$ & $16 \mathrm{~mm}$
\end{tabular}

Before connecting the heart to the perfusion system, it was cut clean of tissue that was not functionally essential, such as fatty or connective tissue and unneeded arteries or veins. This way, the myocardium would be optimally visible for the camera during the experiment.

Already during the in vivo part of the experiment, the water bath and the perfusion solution were heated to $37^{\circ} \mathrm{C}$. After preparing the heart, we connected it to the perfusion system, taking care that no air was left inside the heart or the tubing system. Once inside the bath, we put the MAP electrode into the heart's RV, connected the ECG electrodes and shocking electrodes and let the heart come "back to life" for at least 30 minutes. During this time we started to add blebbistatin to the perfusate (about $7 \mu \mathrm{mol}$ ) in order to reduce optical motion artifacts from muscle contraction. Shortly before the start of the experiment, the voltage sensitive dye di-4-ANEPPS was added (10 to $20 \mathrm{nmol})$.

\subsubsection{Simulating a Physiological Environment}

During the experiment, we kept the heart as close to physiological levels as possible in all relevant parameters. The temperature of the bath and the perfusate was regulated to $37^{\circ} \mathrm{C}$ and the perfusate was continuously bubbled with oxygen and carbon dioxide. 


\begin{tabular}{lrlr}
\multicolumn{2}{c}{ Krebs-Henseleit } & \multicolumn{2}{c}{ Tyrode } \\
$\mathrm{NaCl}$ & $118 \mathrm{mM}$ & $\mathrm{NaCl}$ & $124 \mathrm{~mm}$ \\
$\mathrm{NaHCO}_{3}$ & $25 \mathrm{~mm}$ & $\mathrm{NaHCO}_{3}$ & $24 \mathrm{~mm}$ \\
$\mathrm{KH}_{2} \mathrm{PO}_{4}$ & $1.2 \mathrm{mM}$ & $\mathrm{NaH}_{2} \mathrm{PO}_{4}$ & $0.9 \mathrm{~mm}$ \\
$\mathrm{KCl}$ & $4.7 \mathrm{mM}$ & $\mathrm{KCl}$ & $4 \mathrm{mM}$ \\
$\mathrm{CaCl}_{2}$ & $3.4 \mathrm{mM}$ & $\mathrm{CaCl}_{2}$ & $2 \mathrm{~mm}$ \\
$\mathrm{MgSO}_{4}$ & $1.2 \mathrm{mM}$ & $\mathrm{MgCl}_{2}$ & $0.7 \mathrm{mM}$ \\
D-Glucose & $2 \frac{\mathrm{g}}{\mathrm{L}}$ & D-Glucose & $0.99 \frac{\mathrm{g}}{\mathrm{L}}$
\end{tabular}

TABLE 7.3: Compositions of the solutions used in the Langendorff perfusion experiments. For the pig hearts we used the Krebs-Henseleit, for the dogs Tyrode's solution.

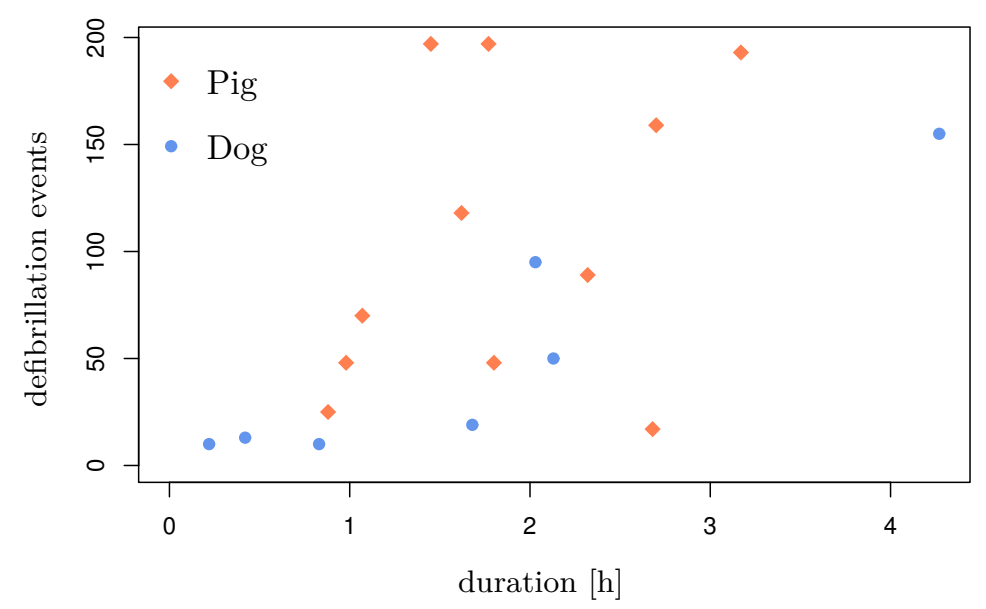

FIGURE 7.6: Experiment durations and number of defibrillation events for the ex vivo experiments. Times measured from the first to the last defibrillation episode. Compare also to figure 6.13

Physiological ion concentrations and a sufficient level of glucose were maintained through perfusion with Krebs-Henseleit solution (for the pig hearts) and Tyrode's solution (for the dog hearts). The respective component concentrations are given in table 7.3 .

During the first ex vivo experiments on dog hearts, unfortunately we did not have a sufficiently big bubble trap at our disposal, so that a few dog hearts suffered from air embolization and the experiment had to be aborted. After we exchanged the bubble trap however, we were able to keep the heart in a healthy state, cf. figure 7.6.

\subsubsection{Defibrillation Energies}

Exactly as in the in vivo experiments, ventricular fibrillation (VF) was induced by lowfield (about 0.5 to $2 \frac{\mathrm{V}}{\mathrm{cm}}$ ), high-frequency (about $50 \mathrm{~Hz}$ ) pulse trains. These pulses were delivered via either the $\mathrm{MAP} /$ catheter electrode or the shock electrodes.

Similarly to the in vivo experiments, we tested the necessary defibrillation energies for LEAP in comparison to the energy values for single shocks. As in [45], we set the total 
pulse length of the biphasic pulses to $8 \mathrm{~ms}$ whenever possible. We evaluated the defibrillation threshold (DFT) by attempting to defibrillate with varying shock energies around the suspected threshold. We repeated the DFT measurement several times during each experiment in order to detect possible drifts and changes in the responsiveness of the heart. While this is a reproduction of the procedure in the in vivo experiments, there is one major difference: If a defibrillation attempt turned out to be unsuccessful, we could simply continue with the next try without delivering a backup shock and waiting for the heart to recover, since the heart was not in danger of ischemia.

We set up the configuration for most of the LEAP shocks as described in [45]: Five rectangular biphasic pulses with a total duration of $8 \mathrm{~ms}$. The pacing frequency $f_{\text {л几 }}$ was chosen either 10 to $20 \%$ above or below the dominant fibrillation frequency $f_{\circlearrowleft}$ as determined from live power spectra (obtained by Fast Fourier Transform, FFT) of the heart's electrical activity. We varied some of these settings, such as the frequency ratio, in order to find the lowest necessary energy.

In the experiments on pig hearts, the shock electrode configuration was as described above in section 7.3.3 except for two days (2012-06-25 and 2012-06-28) when we used one external electrode and an intraventricular catheter as in the in vivo experiments. In the dog heart experiments, we varied the electrical setup in order to reproduce the varying in vivo experiments and to find the electrode configuration which would minimize the required energy for the following open chest experiments. For example, we tested the feasibility of glued electrodes ex vivo first before putting this technique into practice in vivo. Towards the end of the dog experiments, we also switched from separated phases of LEAP and DFT measurements to an interleaved pattern: After each three attempts to terminate VF with LEAP, we inserted one attempt to defibrillate with a single shock, at an energy above or below the previous unsuccessful or successful single shock energy. This DFT measurement pattern allowed us to quickly detect whenever the fibrillation would become more or less vulnerable to shocks.

For some electrode configurations in the pig heart experiments, it was not possible to measure the defibrillation threshold, since the impedance of the perfusion bath setup was too low: It turned out that even at the maximum current of the $1 \mathrm{~kW}$ amplifier $(10 \mathrm{~A})$, the heart could not be defibrillated, neither at the usual pulse widths of 8 to $10 \mathrm{~ms}$ nor at longer pulses of up to $70 \mathrm{~ms}$. Therefore for those experiments, it only makes sense to compare the LEAP thresholds to the lower limit of single pulse DFT defined by said long single pulse energy values. 


\subsubsection{Frequency Scans}

In order to solve the question about which pacing frequency is most beneficial for LEAP success, we systematically varied the pacing frequency $f_{\text {几л }}$ and evaluated the defibrillation success rate for the respective frequency.

After estimating the DFT energy $\hat{E}$, we chose a considerably lower energy $E_{1}<\hat{E}$ for which we still expected some measurable success with LEAP, typically at about $40 \%$ of $\hat{E}$. We attempted to terminate fibrillation with $E_{1}$ at several pacing frequencies $f_{\Omega \Omega}$ at and around the dominant fibrillation frequency $f_{\odot}$. We varied the frequency ratio $f_{\circlearrowleft} / f_{\text {几л }}$ around 1 in steps of 0.1 or 0.05 in both directions until the success rate dropped to near zero. For each of these frequency ratio values, we estimated the success rate by performing three defibrillation attempts. At the latest after the frequency scan, we measured the DFT again, and if it did not change much, repeated the frequency scan for another energy $E_{2}$, higher or lower than $E_{1}$ depending on whether the range of successful frequencies was already very narrow or not.

\subsection{End of Experiment}

As in the in vivo experiments, the experiment continued as long as the heart was in a physiologically stable state. If the efficiency of the dye decreased due to bleaching or if the heart started contracting again, we administered another dose of di-4-ANEPPS or blebbistatin, respectively. Regular monitoring of the DFT gives early evidence of unwanted DFT drift which may distort the perceived energy gain by LEAP. If however fibrillation could be terminated with too low energies, could not be induced anymore or even terminated itself, the experiment was ended. 



\section{Chapter 8}

\section{LEAP Experiment Results}

\subsection{On DFT Measurement}

The measurement of the "defibrillation threshold" (DFT) is notoriously difficult and a topic of many debates [110]. It is routinely estimated for freshly implanted ICDs [111] in order to configure their defibrillation energy, although there are discussions whether this actually is the best practice or if other methods are better suited to customize ICDs (Swerdlow et al. give an overview over ICD calibration methods in [112]).

A common misunderstanding is the perception that the DFT be a fixed property which can be measured with a certain degree of confidence. At the basis of these difficulties lies the probabilistic nature of defibrillation. There is no fixed energy (or electric field or current density, for that matter) below which fibrillation is never terminated and above which defibrillation always succeeds, i.e. no threshold as such. On the contrary, the efficacy of defibrillation is best represented by a dose-response curve, with zero success probability at low energies and an increasing probability for higher amplitudes. The energy with $50 \%$ success rate $E_{50}$ is typically about $10 \mathrm{~J}$ for ICDs and the width between the 20 and $80 \%$ energies is estimated to be $E_{80}-E_{20} \approx 0.6 E_{50}$ [11].

For the application of adjusting the ICD energy the exact dose-response curve is of minor interest. In clinical application settings, it is sufficient to be certain that the set ICD defibrillation energy is high enough to defibrillate in nearly all cases. Exact details of the measurement method notwithstanding, the principle of choosing the ICD energy is always the same. Assume there is a way to get a measured DFT $E_{\mathrm{DFT}}$, which with a high probability (e.g. $95 \%$ ) is at most some chosen energy lower than $E_{99}$. Then increasing 
the DFT by a so-called measured safety margin $E_{\mathrm{M}}$ should result in an energy $E_{\mathrm{ICD}}$ which is an (unknown but positive) intrinsic safety margin above the required $E_{99}{ }^{1}$

The DFT is not defined as a specific feature of e.g. the dose-response curve. Contrariwise, its definition follows purely from a specific measurement protocol, with the objective to keep the variance of $E_{\mathrm{ICD}}$ as low as possible while minimizing potential negative side effects of the measurement on the subject. Among the most popular protocols are (more details in [114]):

Step-Down (alternatively: Step-Up) Start with an expected successful (unsuccessful) energy and decrease (increase) the energy by fixed amounts until defibrillation fails (succeeds) for the first time. The last (finally) working energy is $E_{\mathrm{DFT}}$. This method's lower confidence interval limit can be improved by finding the lowest energy for which defibrillation worked twice or three times in a row (then the method is called DFT + or DFT ++ ), and the initial up/down direction could also be chosen upon the outcome of the first defibrillation attempt.

Binary Search If the distribution of expected dose-response curves is known, it may make sense to search for the DFT by interval bisection. Starting at the average $E_{50}$, the energy is increased or decreased in successively smaller steps depending on the previous defibrillation outcomes.

These protocols claim to find a realistic DFT within typically 3 to 4 (for the binary search) defibrillation attempts or fibrillation episodes. While the results may be sufficient in order to determine a safe $E_{\mathrm{ICD}}$, the error margin is still too large for quantitative comparisons of single pulse and LEAP energies, unless one performs a prohibitively large number of defibrillation attempts, and the outcome may depend on factors like the energy step between tries (see the infobox "Manipulating the DFT").

In our experiments, we typically followed a modified step up/down method: If defibrillation succeeded at one energy, we decreased the energy, else we increased it for the next attempt. Without immediately resulting in a single energy value, this method is guaranteed to have the majority of its direction changes around $E_{50}$. Examining "unequal pairs" of successful and unsuccessful defibrillation attempts therefore allows to directly compare two different defibrillation methods, single pulse and LEAP in the examined case. The Unequal-Pairs method and other approaches to determining defibrillation efficacy are presented in more detail in the appendix, section A.2.

\footnotetext{
${ }^{1}$ Terminology in italics taken from Barold et al. [113] and from Singer and Lang 111.
} 
The sensitivity of the DFT on the measurement protocol
As an example of how the DFT outcome can depend on the choice of protocol parameters,
let us consider the commonly used step down method. Starting with $E^{0}$, the energy is
reduced by $\Delta E$ until defibrillation fails at $E^{k} . E^{k-1}$ is then considered to be the DFT.
$E^{0}$ is usually chosen so that the success probability $\operatorname{Pr}\left(E^{0}\right) \approx 1$, and with a probability
of $p \%$, the DFT will be larger than $E_{100-p}$. For a "wisely chosen" $E^{0}$ and $\Delta E$, the DFT
will be probably close to $E_{50}$, so as to minimize both the risk of delivering too small
shocks in case of fibrillation but also the delivered energy. This choice of $E^{0}$ and $\Delta E$
depends on prior knowledge of the dose-response curve of course though and also imposes
a limit on the accuracy of the DFT result (the width of the probability distribution).
With decreasing $\Delta E$ though, the resulting DFT increases, with the limit being at $E^{0}$
for $\Delta E \rightarrow 0$. This limitation remains valid even for procedures where the DFT is
confirmed by several more defibrillation attempts at the same energy. The practical
implication is that while clinical DFT estimation methods may have their application in
everyday practice to adjust ICD settings in patients, their applicability for quantitative
defibrillation efficacy needs to be thoroughly evaluated.

\subsection{Results of the In Vivo Experiments}

\subsubsection{Evaluation of the Experimental Defibrillator Setup}

We found that our in vivo setup is a valid test bed for assessing clinical defibrillation settings. Except for a single case where the animal died due to prolonged fibrillation episodes (pig, 2012-01-16), the animals showed physiologically stable parameters over the course of the experiment. In all animals but one, fibrillation could be successfully induced by fast local pacing (tip of ICD catheter, 50 to $60 \mathrm{~Hz}$ ) at low voltages. In the experiment where this induction method failed, it turned out that the catheter tip had become detached from the endocardium (pig, 2011-11-28).

In nearly all experimental setups, the energy and current delivered by the experimental defibrillators were sufficient to defibrillate with a single shock. The only exception during the pig experiments was when we tried patch electrodes on the skin in a ventral-dorsal configuration. In this case even the commercial defibrillator we used for backup rescue shocks failed to terminate ventricular fibrillation (2012-02-13). Similarly, at two openchest dog heart experiments, the power amplifiers could not deliver sufficient amounts of current: For the tested electrode configurations, the impedance was too high for the amplifier configuration so that only the backup shocks could finally defibrillate (201303-05 and 2013-03-06).

We acquired control, bioelectrical and other physiological signals at a sampling rate of $2 \mathrm{kHz}$ continuously during each experiment. For each defibrillation attempt, there exists electrophysiological data (ECG or catheter MAP) for the seconds preceding the shocks. 


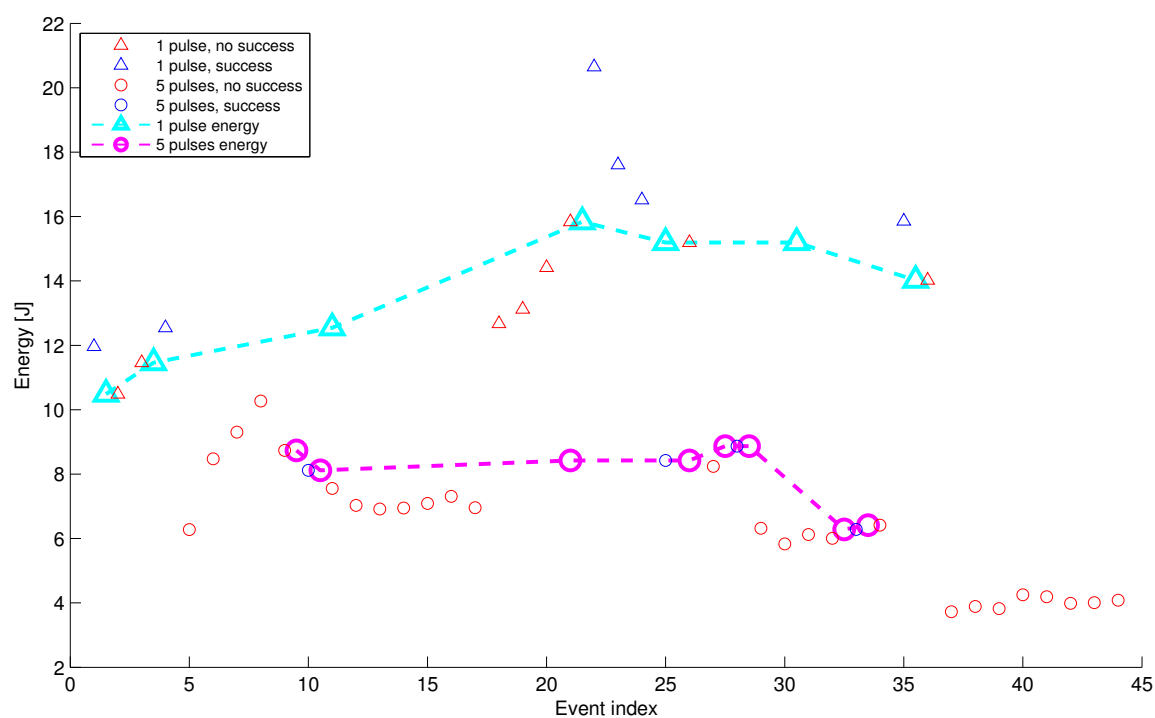

(a) Pig, 2012-02-07

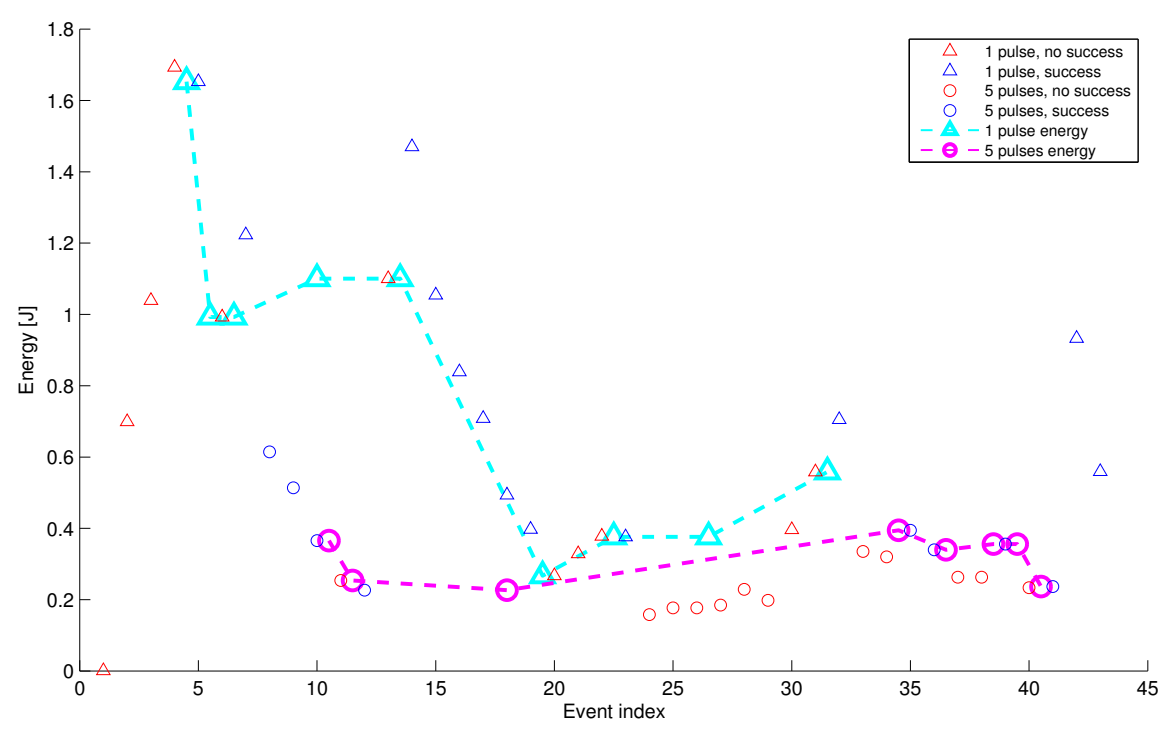

(b) Dog, 2013-03-04

FIgURE 8.1: Energies of two in vivo experiments. The thin symbols represent the single attempts' energies, the thick symbols, connected by dashed lines, are the estimated $E_{50}$ defibrillation energies by the Unequal-Pairs method (conservative).

After the shock, it took the Biopac modules typically a few seconds to recalibrate the internal automatic gain settings.

\subsubsection{Defibrillation Energies}

Figure 8.1 shows the measured energies for the events of two typical experiments. The experiment on 2012-02-07 (figure 8.1a) ran over $2 \mathrm{~h} 39 \mathrm{~min}$, the experiment in March 2013 (figure $8.1 \mathrm{~b}$ lasted for nearly $2 \frac{1}{4} \mathrm{~h}$. The small symbols in the plots show the individual defibrillation attempts, in the order they were performed. The large triangles 
and circles are the typical defibrillation energies, estimated by the Unequal-Pairs method as described in the previous section and in A.2. Here the more conservative approach was used, where for single shocks the lower and for LEAP the higher energy was taken. The dog experiment shows a behavior typical for experiments of longer duration, strong fluctuations of the dose-response curve. While at the beginning the defibrillation threshold seemed to be around $1 \mathrm{~J}$, half of that value was sufficient later in the experiment to reliably defibrillate.

The energies for all in vivo experiments and the $E_{50}$ estimate are shown in figure 8.28 .5 . The energies were in the expected range for the given electrode configurations, about 15 to $25 \mathrm{~J}$ for ICDs and 0.5 to $3 \mathrm{~J}$ for the mesh electrodes. The exact values differed from case to case, probably due to different electrode configurations and animal age and weight. 


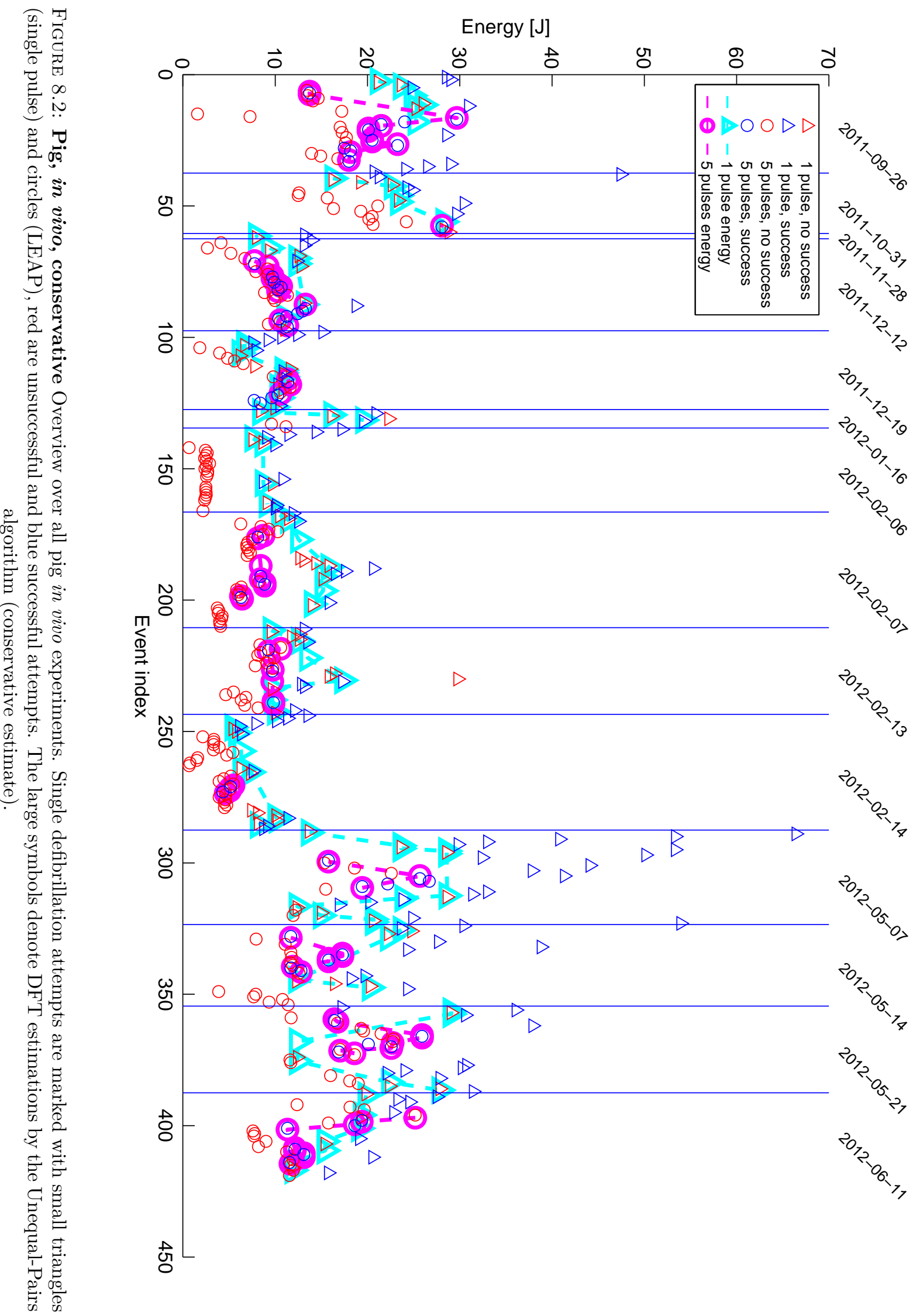




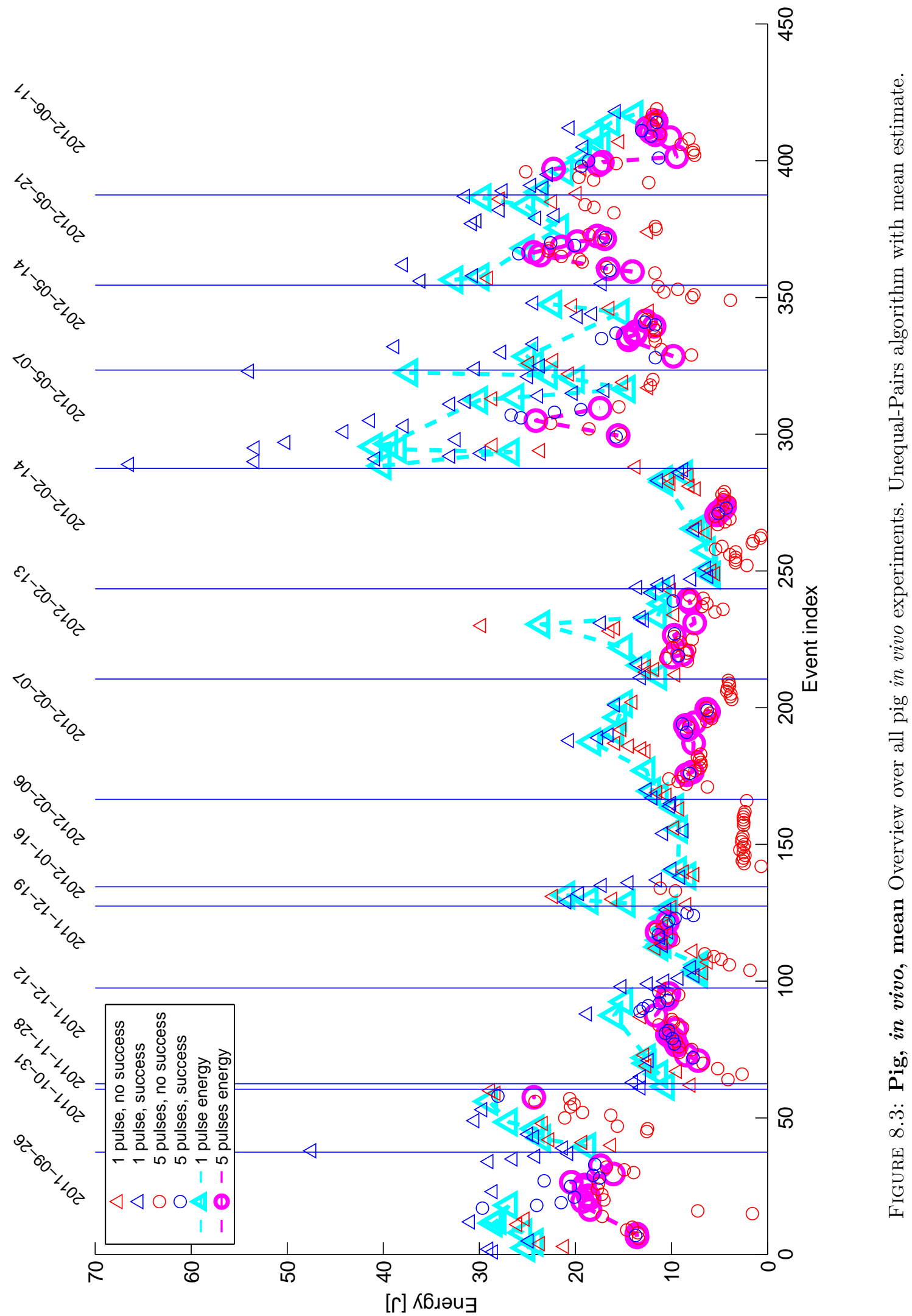




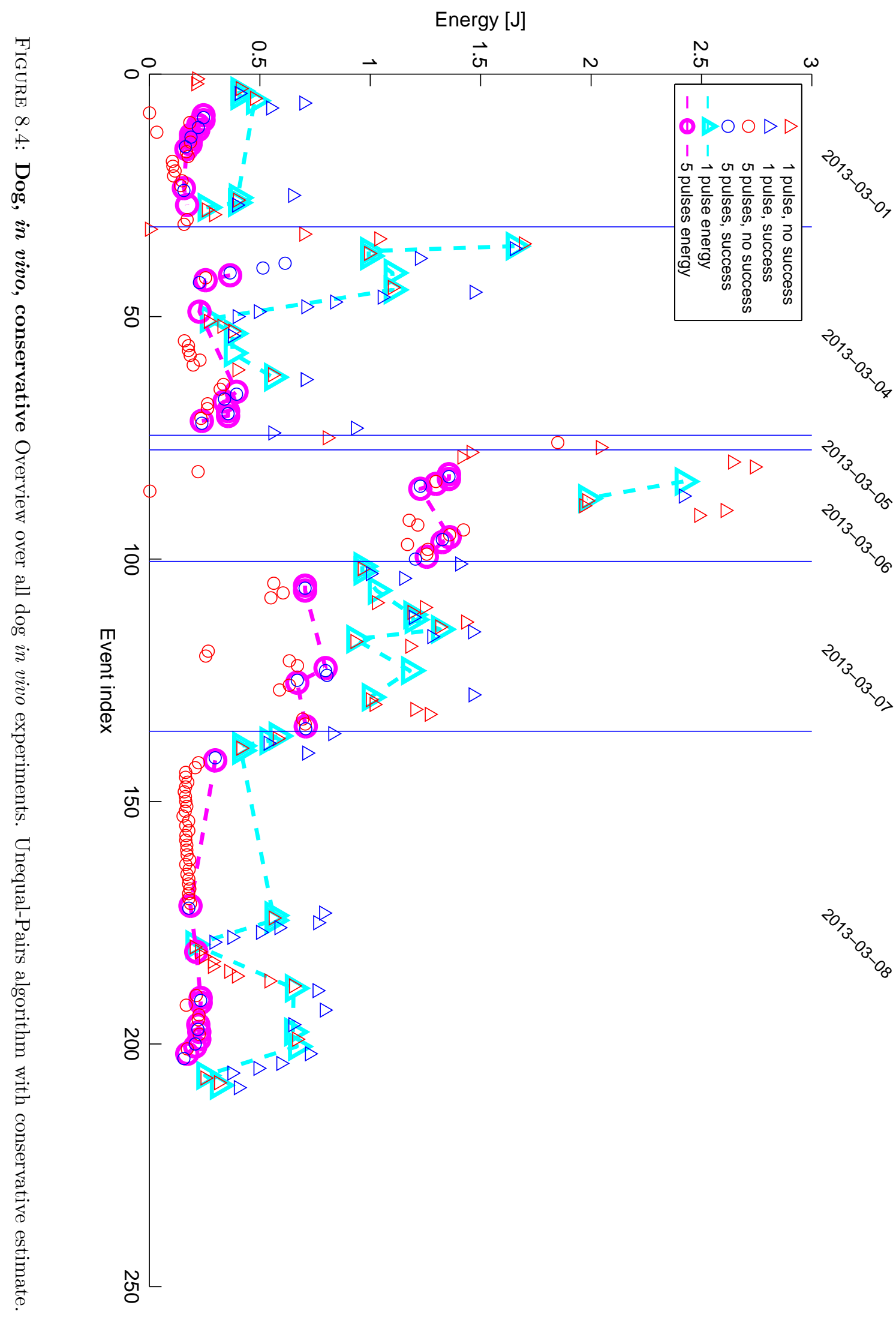




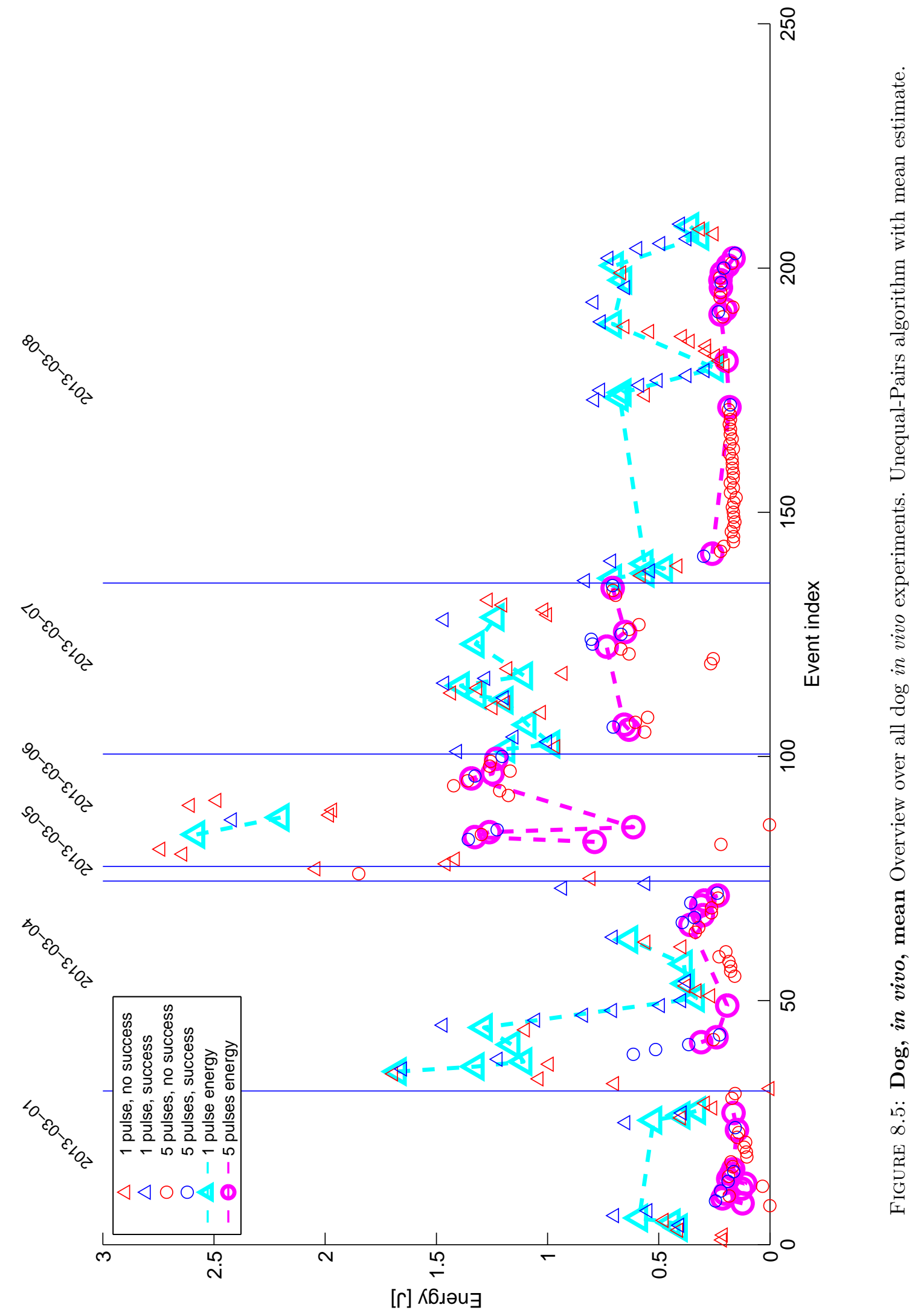




\subsubsection{LEAP Efficiency (In Vivo)}

For all in vivo experiments, we calculated the mean energy $\left\langle E_{\Omega}\right\rangle$ required for single pulses and the mean and minimum energies $\left\langle E_{\Omega \Omega}\right\rangle$ and $E_{\Omega \Omega m i n}$ which were sufficient when pacing with 5 pulses.

These energy estimates, for the same examples as above, are shown in figure 8.6. As in most experiments, the mean estimated energies were significantly lower for LEAP than for single pulses. The energy gains for all in vivo experiments, $\frac{\left\langle E_{\Omega \Omega}\right\rangle}{\left\langle E_{\Omega}\right\rangle}$ and $\frac{E_{\Omega \Omega m i n}}{\left\langle E_{\Omega}\right\rangle}$, are plotted in figure 8.6 and shown in the following table:

\begin{tabular}{l|cccccl} 
Experiment & $\left\langle E_{\Omega}\right\rangle[\mathrm{J}]$ & $\frac{\left\langle E_{\Omega \Omega}\right\rangle}{\left\langle E_{\Omega}\right\rangle}$ & $\frac{E_{\Omega \Omega \min }}{\left\langle E_{\Omega}\right\rangle}$ & $\left.\frac{\left\langle E_{\Omega \Omega}\right\rangle}{\left\langle E_{\Omega}\right\rangle}\right|_{95 \%}$ & significance: $p$ \\
\hline Pig, 2011-09-26 & 26.4 & 0.67 & 0.51 & 0.74 & $* * *$ & $3.3 \times 10^{-7}$ \\
Pig, 2011-12-12 & 13.4 & 0.72 & 0.54 & 0.86 & $* *$ & 0.0055 \\
Pig, 2011-12-192 & 9.59 & 1.12 & 1.09 & 1.26 & & 0.95 \\
Pig, 2012-02-07 & 14.3 & 0.53 & 0.43 & 0.66 & $* * *$ & 0.0001 \\
Pig, 2012-02-13 & 13.8 & 0.63 & 0.55 & 0.88 & $*$ & 0.013 \\
Pig, 2012-02-14 & 7.86 & 0.62 & 0.57 & 0.80 & $* *$ & 0.0020 \\
Pig, 2012-05-07 & 30.6 & 0.62 & 0.51 & 0.86 & $* *$ & 0.009 \\
Pig, 2012-05-14 & 21.7 & 0.60 & 0.45 & 0.84 & $*$ & 0.012 \\
Pig, 2012-05-21 & 27.3 & 0.71 & 0.51 & 0.85 & $* *$ & 0.0016 \\
Pig, 2012-06-11 & 18.5 & 0.74 & 0.51 & 0.91 & $* *$ & 0.0077 \\
\hline Dog, 2013-03-01 & 0.45 & 0.36 & 0.25 & 0.54 & $* * *$ & 0.00021 \\
Dog, 2013-03-04 & 0.92 & 0.30 & 0.21 & 0.64 & $* *$ & 0.0023 \\
Dog, 2013-03-06 & 2.4 & 0.47 & 0.26 & 0.76 & $*$ & 0.019 \\
Dog, 2013-03-07 & 1.2 & 0.56 & 0.53 & 0.63 & $* * *$ & $1.7 \times 10^{-7}$ \\
Dog, 2013-03-08 & 0.55 & 0.38 & 0.30 & 0.53 & $* * *$ & $7.0 \times 10^{-6}$ \\
\hline \hline Pig, mean( $\pm 47.5 \%)^{2}$ & & $0.65(5)$ & $0.51(3)$ & & & \\
Dog, mean( $\pm 47.5 \%)^{2}$ & & $0.41(13)$ & $0.31(16)$ & & &
\end{tabular}

Statistical significance ${ }^{3}$ in this table is denoted by one, two or three asterisks, which stand for a p-value smaller than $0.05,0.01$ and 0.001 , respectively; the $5^{\text {th }}$ column shows the upper limit of the $95 \%$ confidence interval. On average over all dog experiments, with LEAP the energy could be reduced to $41 \%$, with an average "best energy per experiment" of $31 \%$. The numbers in parentheses in the last two rows give the distance of the mean to the limits of the $95 \%$ confidence interval.

\footnotetext{
${ }^{2}$ In the experiment of 2011-12-19, the single pulse energy increased significantly by $50 \%$ before the first LEAP energies could be estimated. Therefore this experiment's energy gain values were ignored for the mean.

${ }^{3}$ In the difference of energy means, evaluated by a one-sided t-test.
} 


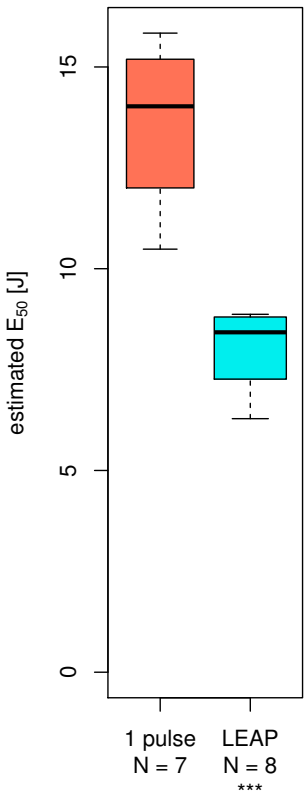

(a) Pig, 2012-02-07

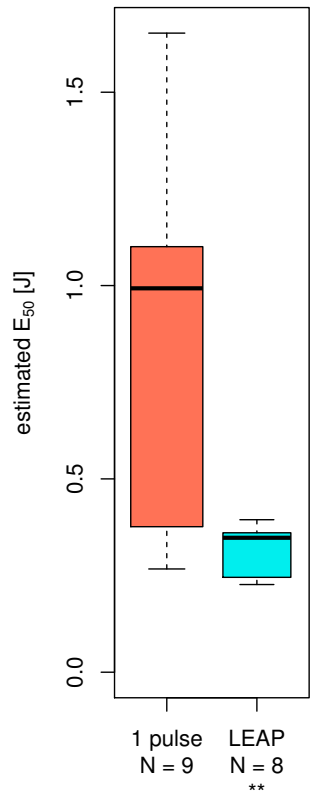

(b) Dog, 2013-03-04

FIGURE 8.6: Energy estimates (conservative) of the in vivo experiments shown in figure 8.1. Two asterisks $(* *)$ denote statistical significance of means of $p<0.01$, three $(* * *)$ mean $p<0.001$. Here the mean gain in the energy required for defibrillation is $41 \%$ (pig) and $62 \%(\mathrm{dog})$.

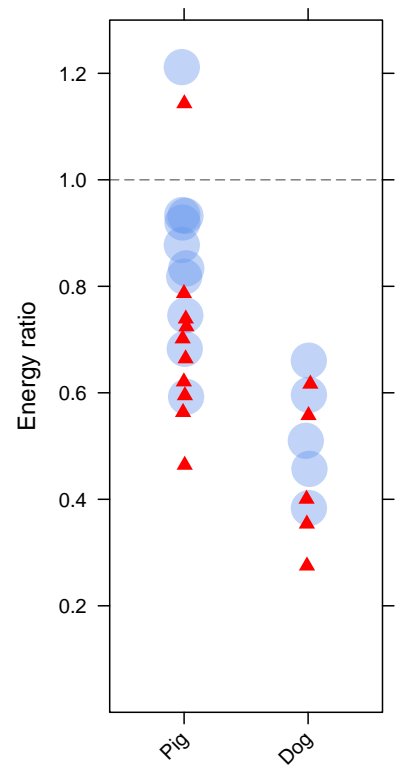

(a) Conservative estimate

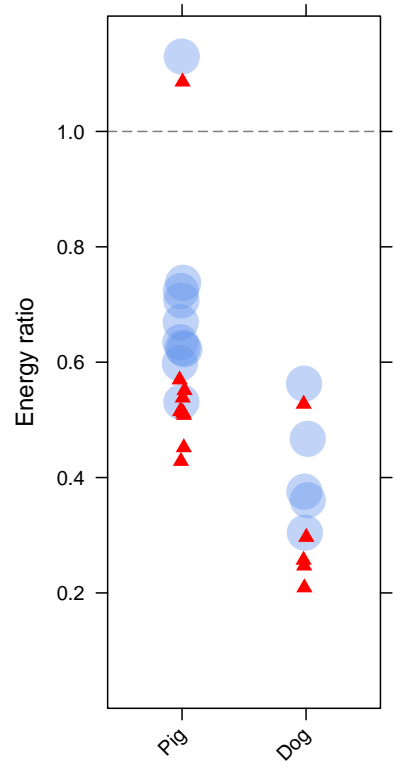

(b) Mean estimate

FIGURE 8.7: Energy gains through LEAP in all in vivo experiments with results for both single pulse and LEAP defibrillation attempts. Mean LEAP energies (as a fraction of the to single pulse energies) are denoted as blue circles, the minimum LEAP energies which were found in each experiment are shown as red triangles. 


\subsubsection{Frequency Dependency of LEAP Success}

Due to the limited number of defibrillation events in the in vivo experiments and the drift in the single pulse DFT, it was not possible to exactly determine the optimum pacing frequency $f_{\Omega \text { 几 }}$ in relation to the dominant fibrillation frequency $f_{\Im}$.

In figure 8.8, we pooled pooled all events from the in vivo experiments, separately for dogs and pigs. Instead of the total energy, which varied depending on the configuration, we normalized it by the mean of the estimated single pulse energies $\left(\hat{e}:=\frac{E_{\Omega \Omega}}{\left\langle E_{\Omega}\right\rangle}\right)$. The ratio of successful vs. unsuccessful attempts at such an energy and the projected frequency ratio $\left(\hat{f}:=\frac{f_{n \Omega}}{f_{\oplus}}\right)$ is presented through differently sized circles, the area is proportional to this success rate. Since the average single pulse energy was not constant over the course of an experiment (see the next section) and here $\hat{f}$ only represents the frequency ratio we targeted during the experiment, the exact number may vary for each event. Nevertheless, this method allowed us to gain information from as many data sources as possible.

In the pig experiments, we could not find a preference for a specific frequency ratio. This result is in line with the frequency-success plots for each single experiment, shown in the appendix in section B.2. The dog experiments, in contrast (figure 8.8b) indicate a preference for frequency ratios $<1$ at low to medium energies.

In the dog experiments, for those energies with at least three events on either frequency side, the estimated average success rates $\mathrm{Pr}$ are:

\begin{tabular}{r|rr}
$\hat{e}$ & $\operatorname{Pr}(\hat{f}<1)$ & $\operatorname{Pr}(\hat{f}>1)$ \\
\hline 0.5 & 0.50 & 0.18 \\
0.3 & 0.47 & 0.33 \\
0.2 & 0.21 & 0.063
\end{tabular}

As evaluated with a paired t-test, given these measured probabilities, with $p=95 \%$ the success rate of overdrive pacing $(\hat{f}>1)$ is at most 5 percentage points higher than underdrive pacing $(\hat{f}<1)$. This suggests that underdrive LEAP may indeed be more efficient than overdrive pacing.

\subsubsection{Reproducibility}

The energy reduction values from section 8.1.3 (LEAP Efficiency (In Vivo) were possibly influenced by one factor: drifting of the single pulse DFT over the course of the experiment. In some experiments, the energy estimates did not stay nearly constant as expected, but exhibited sudden jumps in either direction or drifted towards considerably 


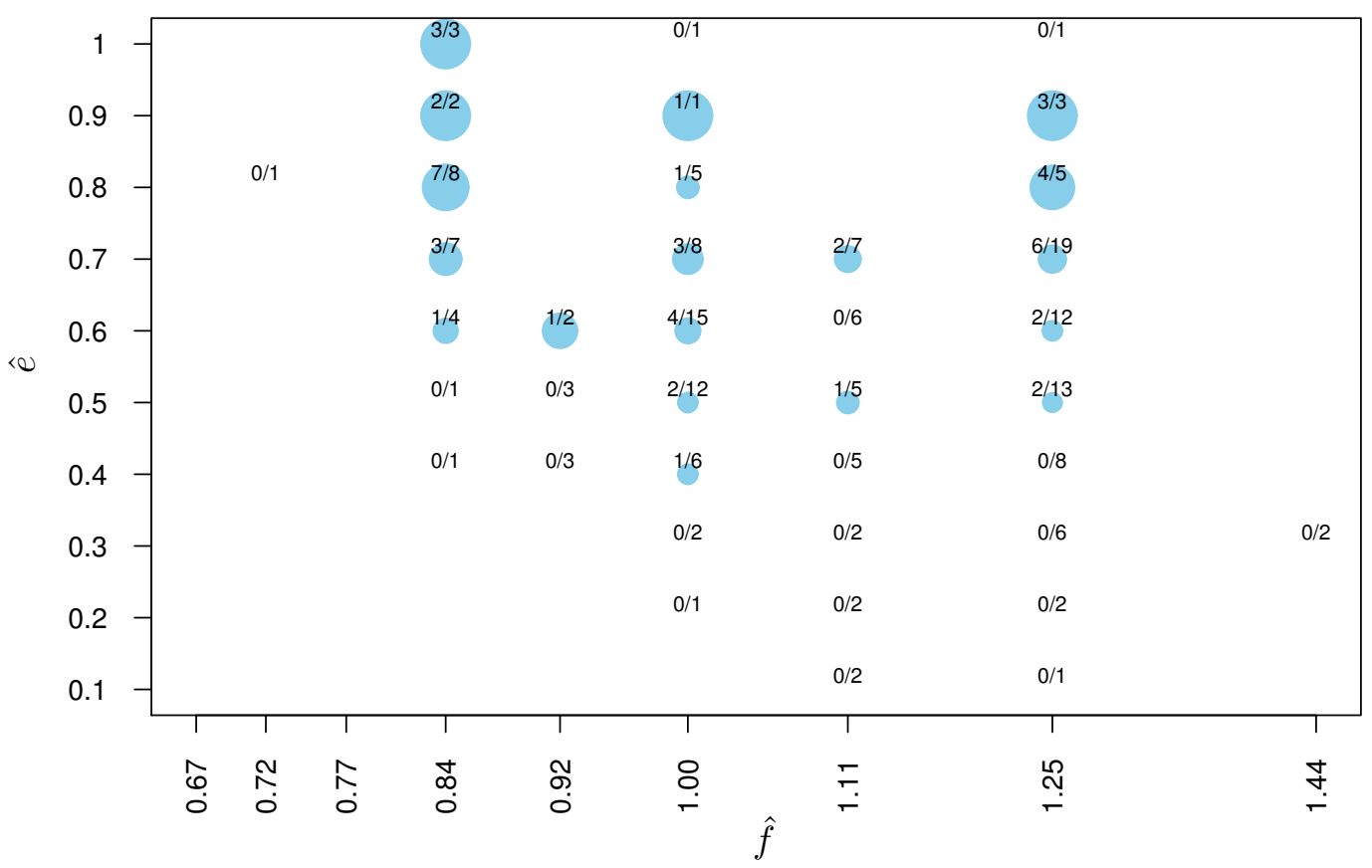

(a) Pig in vivo defibrillation probabilities

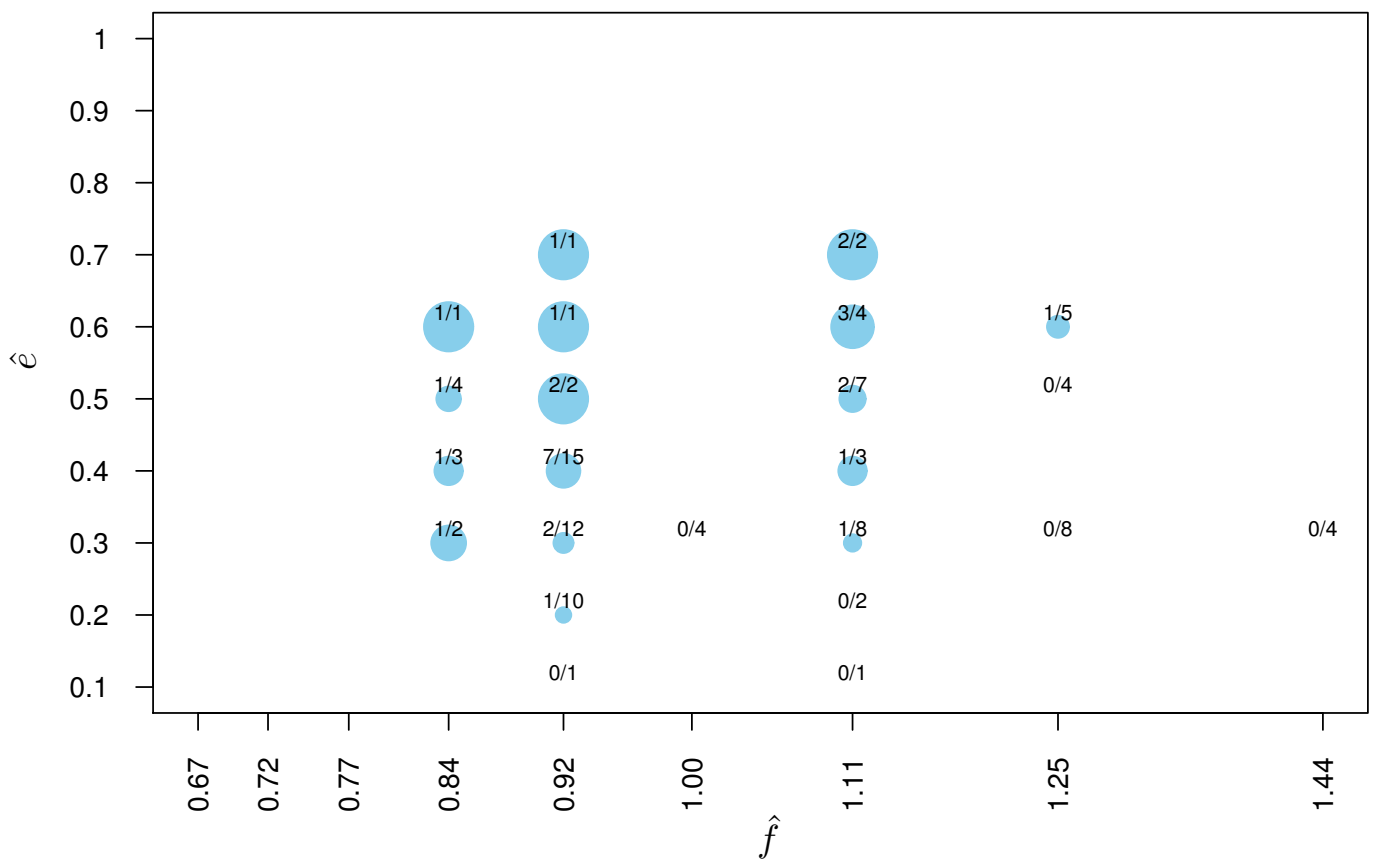

(b) Dog in vivo defibrillation probabilities

FiguRE 8.8: Defibrillation probabilities for LEAP at different frequencies and energies. The area of the circles corresponds to the success ratio which is also given as numbers (success/total attempts) above the symbols. For frequency-energy configurations without numbers, no data is available. Only for the dog experiments (bottom plot) there seems to be a consistent preference for a distinct frequency range, lower pacing frequencies $\left(f_{\Omega \Omega}<f_{\circledast}\right)$ in this case. For all energies of at most half the single pulse energy and with at least three events on either side of $\hat{f}=1$, the lower frequencies have consistently higher success probabilities. 
higher or lower values. When this happened suddenly after a long running experiment, we assumed the animal was in an irrecoverably pathological state and terminated the experiment.

The most prominent examples of irregular DFT estimates, with no apparent reasons for this behavior, are:

\begin{tabular}{|c|c|c|}
\hline Experiment & DFT energies & Comment \\
\hline Pig, 2011-10-31 & $\begin{array}{l}\text { rising continuously from } 18.8 \\
\text { to } 29.0 \mathrm{~J}\end{array}$ & rise by $>50 \%$ \\
\hline Pig, 2012-06-11 & $\begin{array}{l}\text { falling continuously from } 23.7 \\
\text { to } 13.9 \mathrm{~J}\end{array}$ & decline by $\approx 40 \%$ \\
\hline Dog, 2013-03-04 & $\begin{array}{l}\text { mean DFT first around } 1.3 \mathrm{~J} \text {, } \\
\text { later around } 0.43 \mathrm{~J}\end{array}$ & $\begin{array}{l}\text { p-value after Student's t test: } \\
p<0.0005\end{array}$ \\
\hline Dog, 2013-03-08 & $\begin{array}{l}\text { in the middle of experiment: } \\
\text { drop of DFT from around } \\
0.58 \mathrm{~J} \text { to } 0.25 \mathrm{~J} \text { and immediate } \\
\text { rise to the old values again }\end{array}$ & $\begin{array}{l}\text { rise to "regular" values af- } \\
\text { ter DFT drop may have } \\
\text { been caused by increased rest- } \\
\text { ing times between fibrillation } \\
\text { episodes }\end{array}$ \\
\hline
\end{tabular}

If unexpected changes similar to these happened during LEAP attempts, they may have biased the LEAP efficiency values without being detected.

The increased resting time between fibrillation episodes during the last two dog experiments did not show conclusively that the single shock DFT would become more or less stable over the experiment or if the resting time has any influence on the comparability of different experiments. It allowed however to record at least as many events (2013-03$07, n=38)$ or even significantly more $(2013-03-08, n=77)$ compared to shorter resting times.

We performed LEAP experiments on pigs and dogs, albeit with different electrode configurations. Therefore direct comparison of achieved results is neither possible nor necessary. There were no noticeable differences for the energy requirements between the pig races we used. 


\subsection{Results of the Ex Vivo Experiments}

\subsubsection{Setup Evaluation and Comparison to In Vivo}

For pig and dog heart experiments, the ex vivo setups were designed to reproduce the respective in vivo environment as far as possible. The impedances were significantly lower than in vivo though (c.f. table 7.1), therefore we used power amplifiers for nearly all experiments instead of our custom-built defibrillator prototype. We found that generally the same methods for VF induction and termination could be applied in vivo and ex vivo and yielded comparable results, both for far-field electrodes (pig) and epicardial patches (dog).

Electrical and electrophysiological data was acquired with the same combination of software and hardware as in vivo and with the same restrictions imposed by the automatic calibration after shocks. Additionally we acquired fluorescence imaging movies for most events, which do not suffer from this type of limitation and give immediate feedback for the heart's activation state during and after the shocks.

Owing to the low impedances and large volumes of water around the heart, for some hearts the energy requirement for single pulses exceeded the capabilities of our power amplifiers. Therefore on six experiments, the defibrillation energy data could not be normalized by the respective single pulse DFT.

\subsubsection{Defibrillation Energies}

Using the same notation as in section 8.1.2, the energies for all ex vivo experiments are plotted in figure 8.9-8.14. Depending on the configuration, the typical single pulse energies ranged from under $0.5 \mathrm{~J}$ to some value above $45 \mathrm{~J}$ (higher than what could be provided by the amplifiers). The necessary LEAP energies were consistently at or below the single pulse energies, down to below $0.2 \mathrm{~J}$. As expected, the in vivo and ex vivo energies were comparable for the dog heart experiments, since the electrode configurations were virtually identical. 


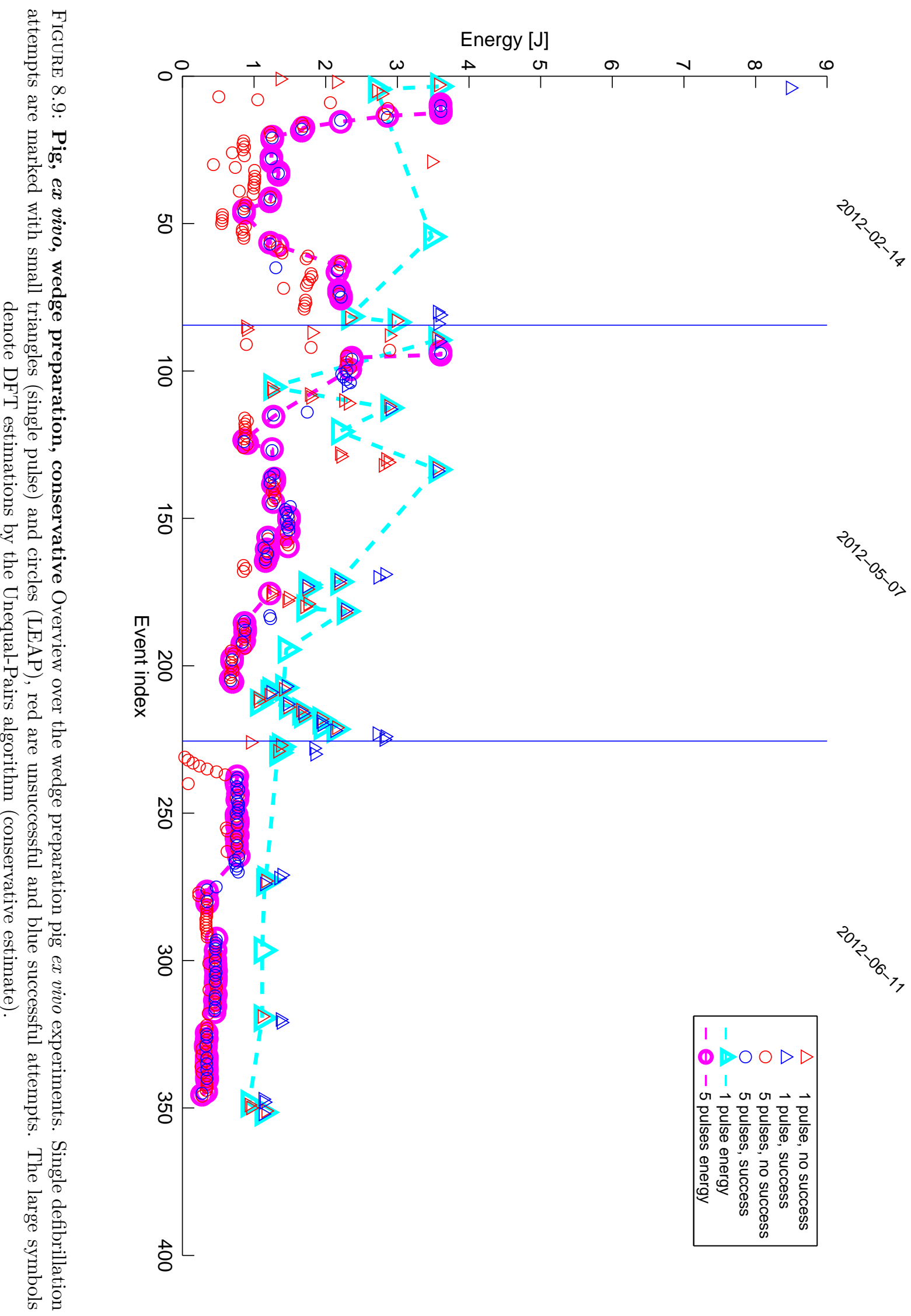




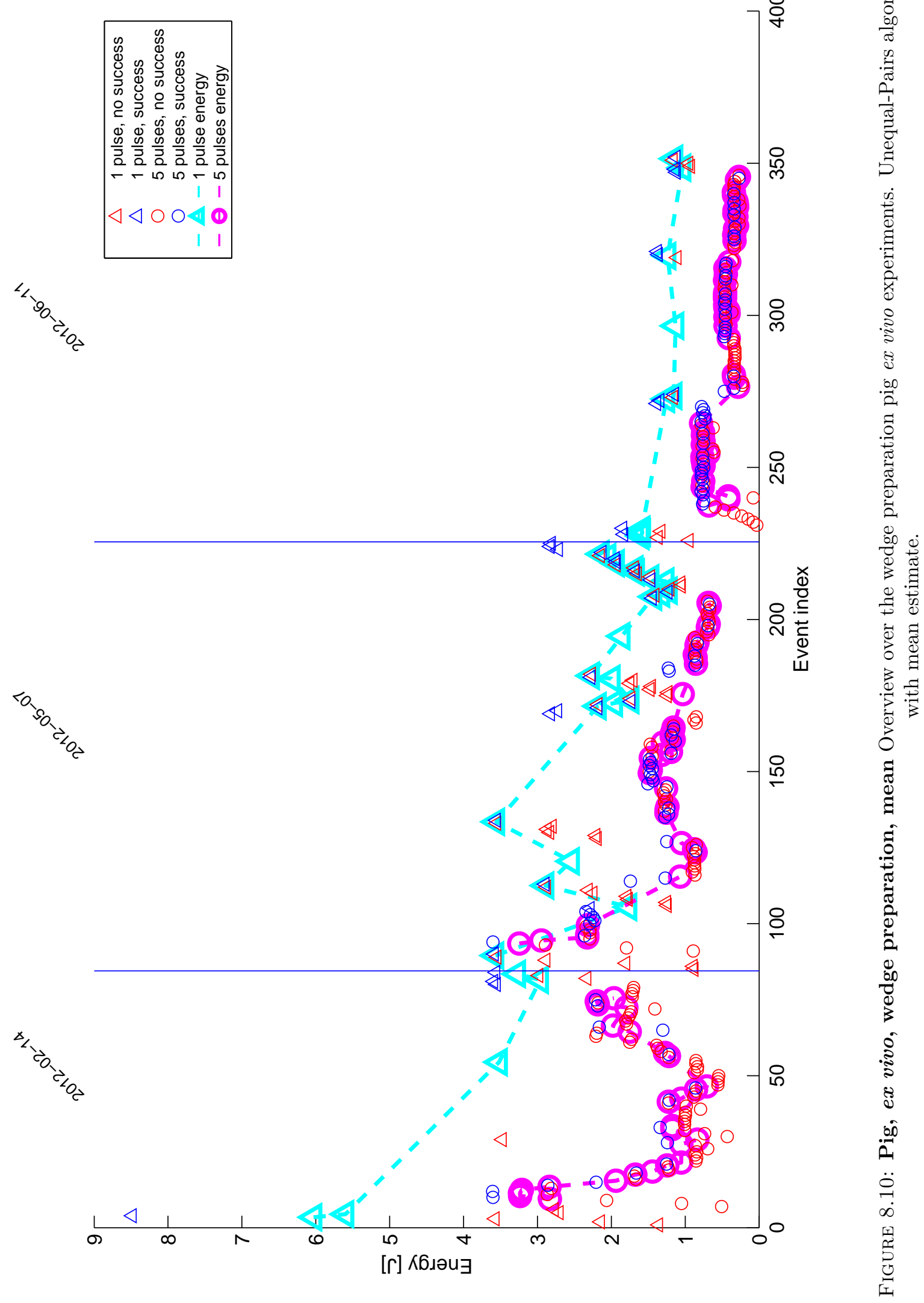




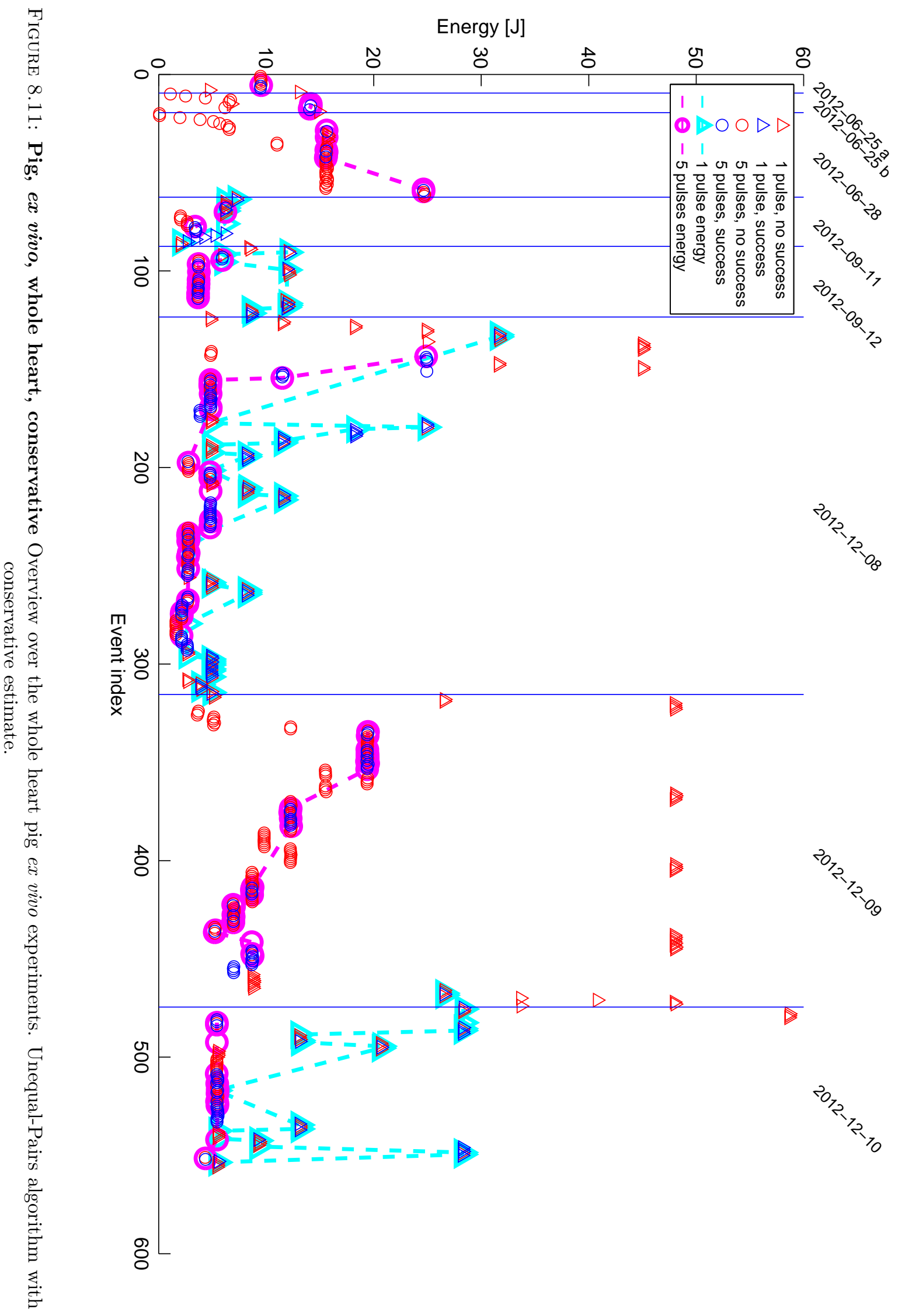




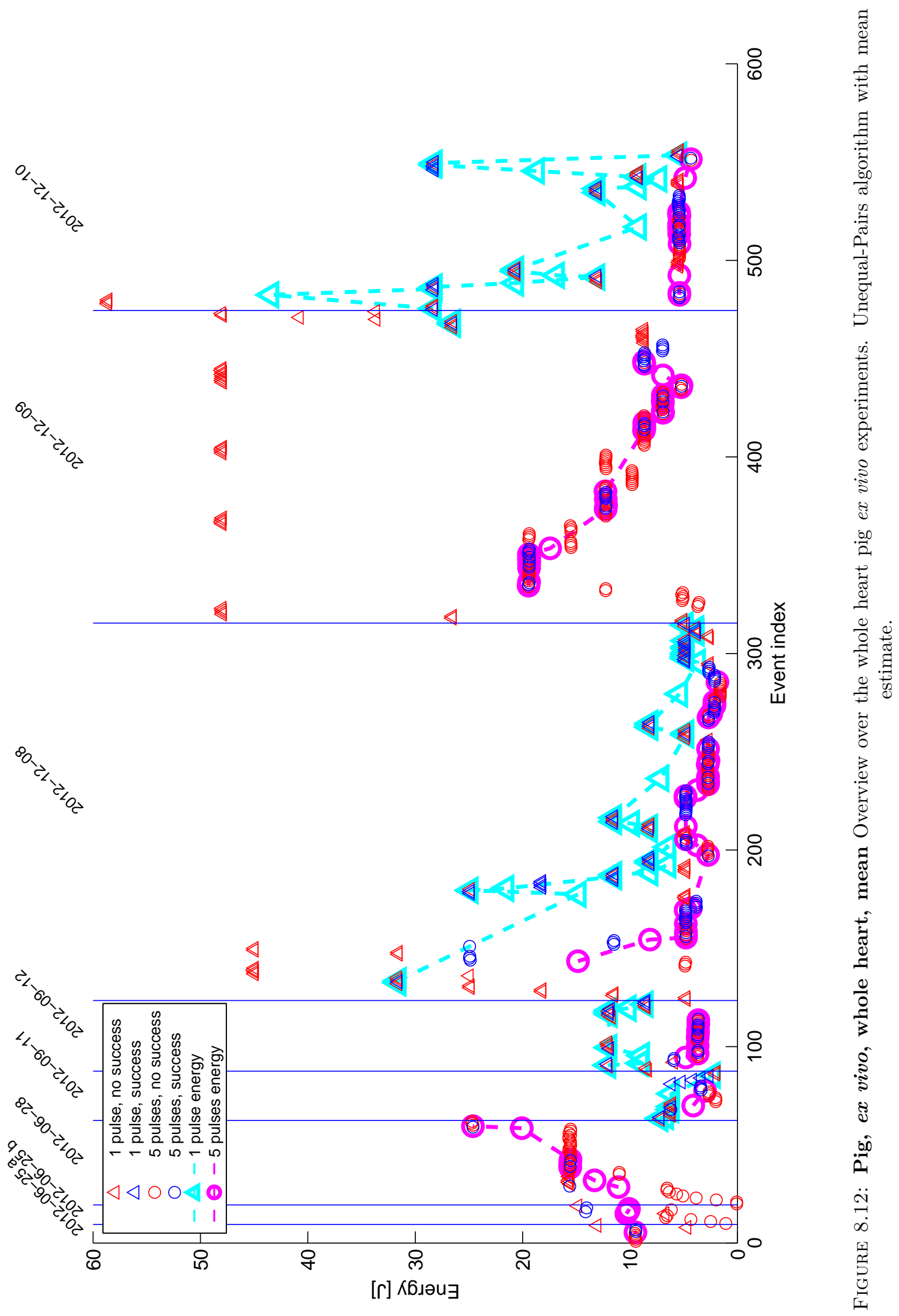




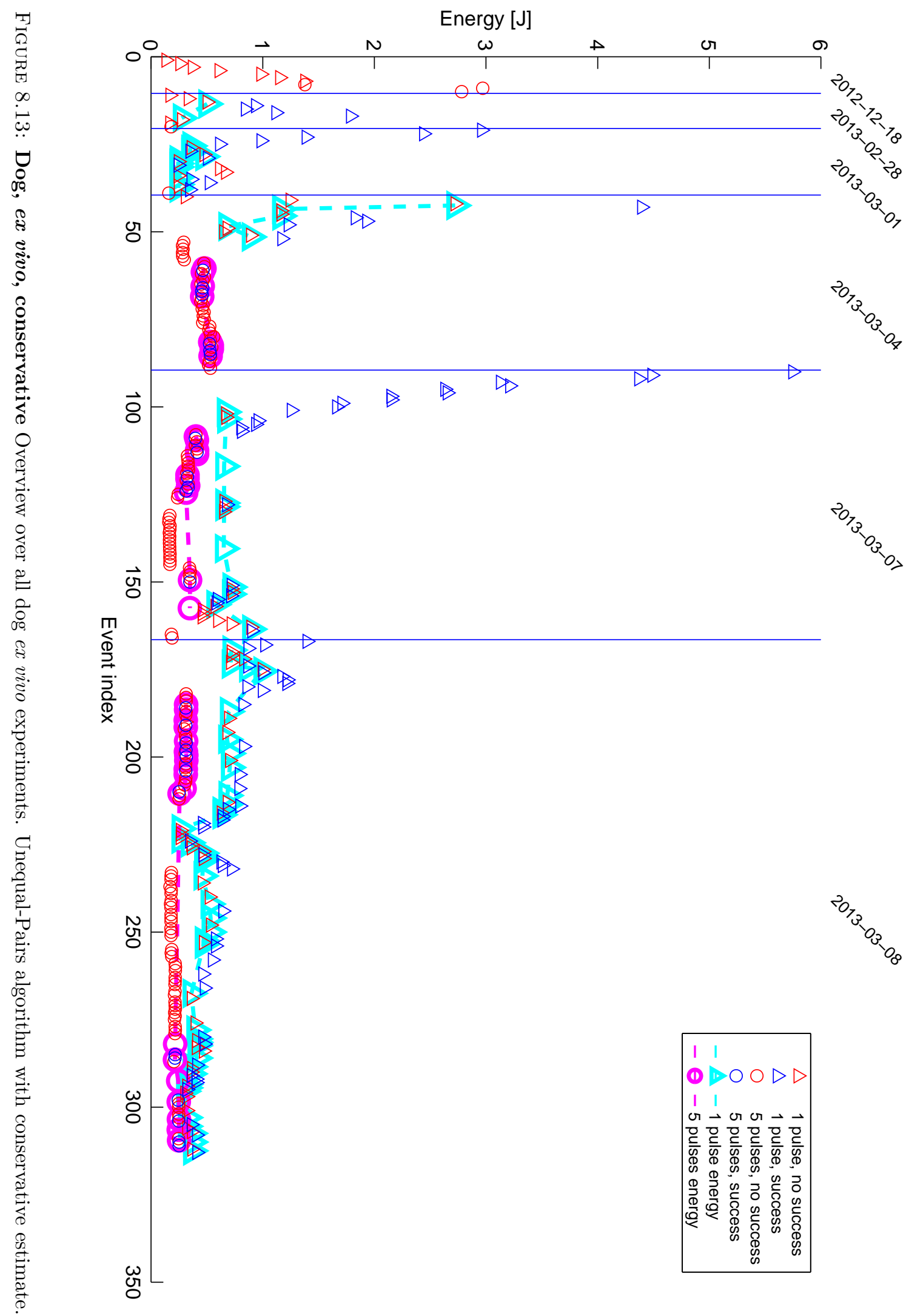




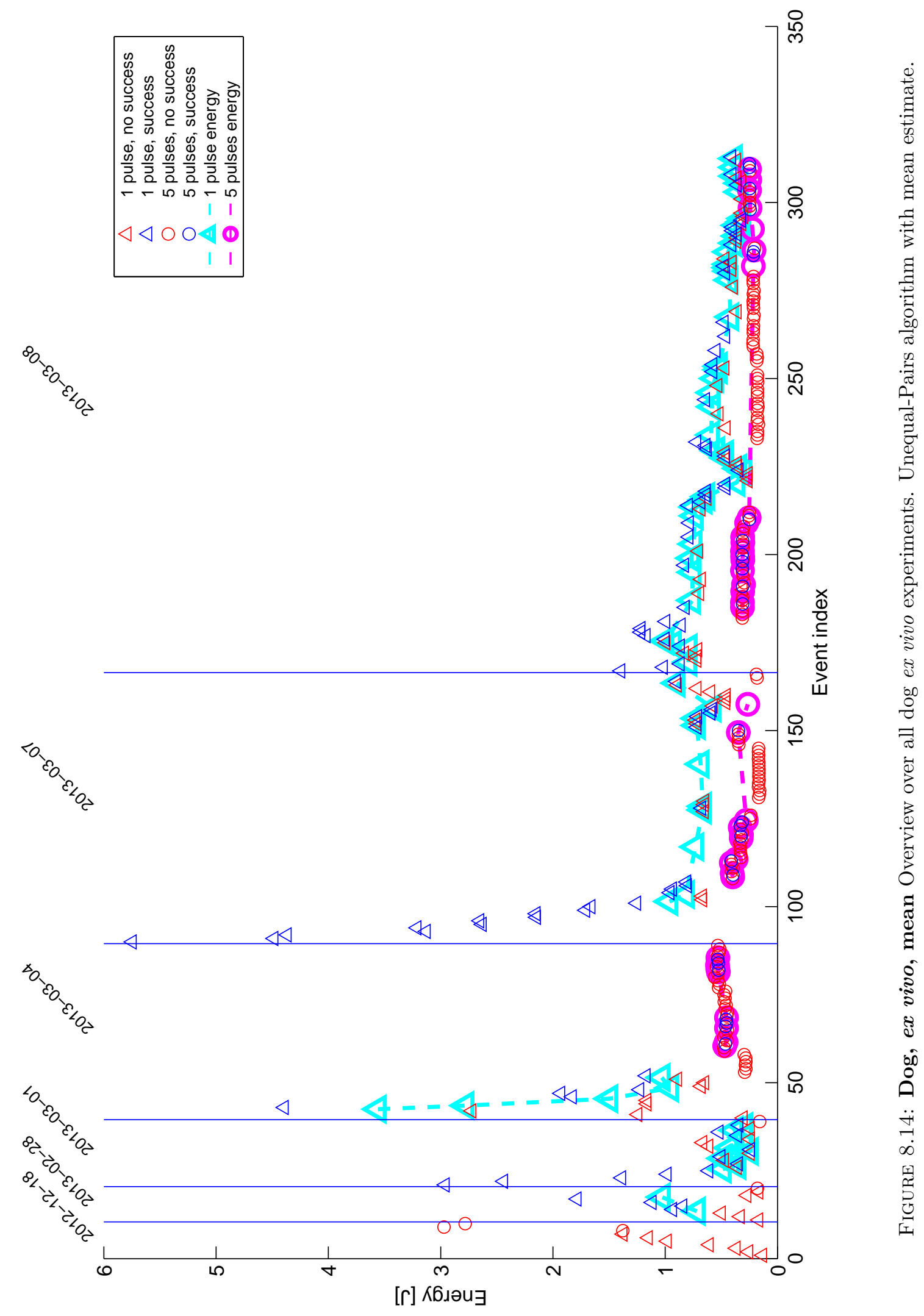




\subsubsection{LEAP Efficiency (Ex Vivo $)$}

For each experiment, we calculated the energy distributions of single pulse and LEAP energy estimates, always averaged over a whole experiment. Figure 8.15 exemplarily shows the energies for two experiments with comparatively many measurements. As in the in vivo experiments, the LEAP energies were significantly lower than the single pulse energies. Figure 8.16 shows the average and maximum gain achieved in the ex vivo experiments.

A comparison of the LEAP energy gains is given in the following table, for those experiments with successful and unsuccessful defibrillation attempts with one and five pulses.

\begin{tabular}{|c|c|c|c|c|c|c|}
\hline Experiment & $\left\langle E_{\Omega}\right\rangle[\mathrm{J}]$ & $\frac{\left\langle E_{\Omega \Omega}\right\rangle}{\left\langle E_{\Omega}\right\rangle}$ & $\frac{E_{\Omega \Omega \min }}{\left\langle E_{\Omega}\right\rangle}$ & $\left.\frac{\left\langle E_{\Omega \Omega}\right\rangle}{\left\langle E_{\Omega}\right\rangle}\right|_{95 \%}$ & \multicolumn{2}{|c|}{ significance: $p$} \\
\hline Pig, 2012-02-14 & 4.28 & 0.41 & 0.17 & 0.72 & $* *$ & 0.0072 \\
\hline Pig, 2012-05-07 & 2.02 & 0.64 & 0.33 & 0.79 & $* * *$ & 0.00011 \\
\hline Pig, 2012-06-11 & 1.30 & 0.37 & 0.21 & 0.48 & $* * *$ & $5.4 \times 10^{-7}$ \\
\hline Pig, 2012-09-11 & 5.79 & 0.61 & 0.52 & 0.93 & * & 0.030 \\
\hline Pig, 2012-09-12 & 10.7 & 0.36 & 0.34 & 0.45 & $* * *$ & $1.5 \times 10^{-7}$ \\
\hline Pig, 2012-12-08 & 9.45 & 0.43 & 0.20 & 0.66 & $* * *$ & $8.4 \times 10^{-5}$ \\
\hline Pig, 2012-12-094 & 26.5 & 0.46 & 0.20 & 0.52 & $* * *$ & $1.9 \times 10^{-14}$ \\
\hline Pig, 2012-12-10 & 19.1 & 0.28 & 0.23 & 0.48 & $* * *$ & $4.7 \times 10^{-6}$ \\
\hline Dog, 2013-03-04 & 1.96 & 0.25 & 0.23 & 0.81 & $*$ & 0.023 \\
\hline Dog, 2013-03-07 & 0.744 & 0.46 & 0.36 & 0.56 & $* * *$ & $1.4 \times 10^{-7}$ \\
\hline Dog, 2013-03-08 & 0.534 & 0.52 & 0.39 & 0.61 & $* * *$ & $3.7 \times 10^{-11}$ \\
\hline $\operatorname{Pig}, \operatorname{mean}( \pm 47.5 \%)^{4}$ & & $0.44(13)$ & $0.28(11)$ & & & \\
\hline Dog, mean $( \pm 47.5 \%)^{\sqrt{4}}$ & & $0.41(35)$ & $0.33(21)$ & & & \\
\hline
\end{tabular}

The indication of statistical significance of the difference of means of single pulse and LEAP defibrillation follows the convention of one to three asterisks for successively smaller p-values (smaller than 0.05, 0.01 and 0.001). In all experiments, LEAP was significantly more efficient than single pulse defibrillation, with up to $83 \%$ energy gain; on average about $70 \%$ energy gain was possible.

The same restrictions regarding interpretation of the exact energy reduction numbers apply as for the in vivo experiments: also ex vivo the single pulse energies which were used for normalization of the defibrillation energies showed similarly fluctuating behavior. 


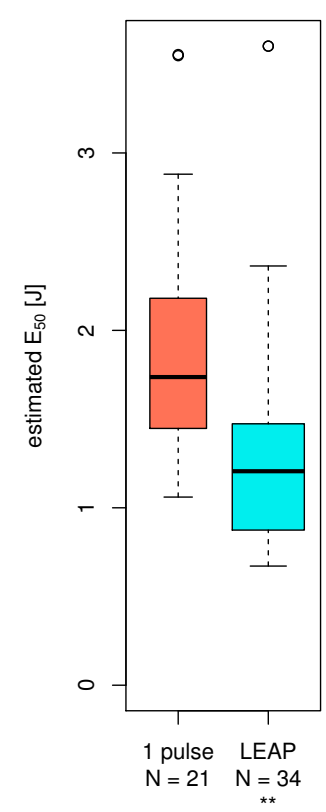

(a) Pig, 2012-05-07

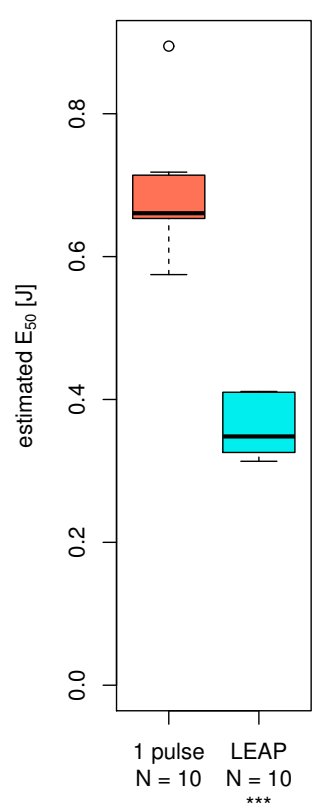

(b) Dog, 2013-03-07

FiguRe 8.15: Energy estimates (conservative) of two ex vivo experiments, on 201205-07 and 2013-03-07. Two asterisks $(* *)$ denote statistical significance of means of $p<0.01$, three $(* * *)$ mean $p<0.001$. With the used conservative estimate the mean gain in the energy required for defibrillation is at least $44 \%$ (pig) and $51 \%(\mathrm{dog})$.

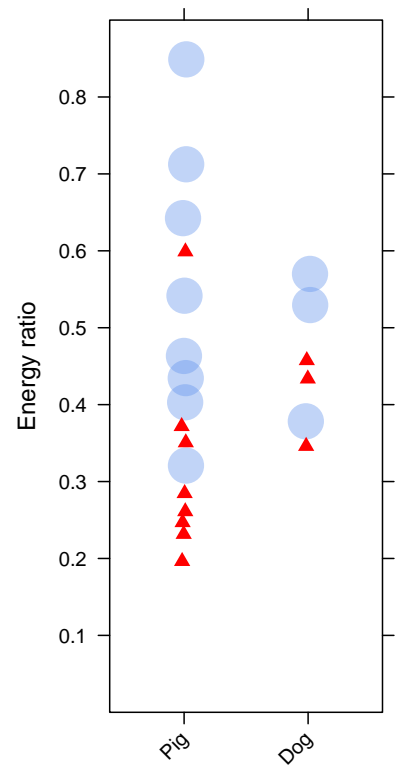

(a) Conservative estimate

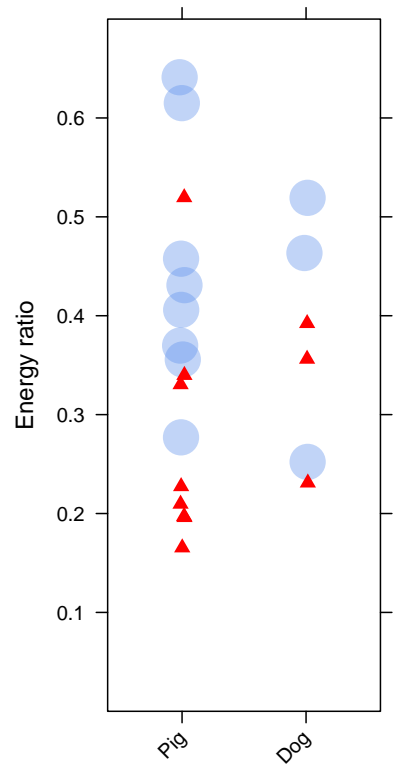

(b) Mean estimate

FigURE 8.16: Energy gains through LEAP in all ex vivo experiments with results for both single pulse and LEAP defibrillation attempts. Mean LEAP energies (as a fraction of the single pulse energies) are denoted as blue circles, the minimum LEAP energies which were found in each experiment are shown as red triangles. 


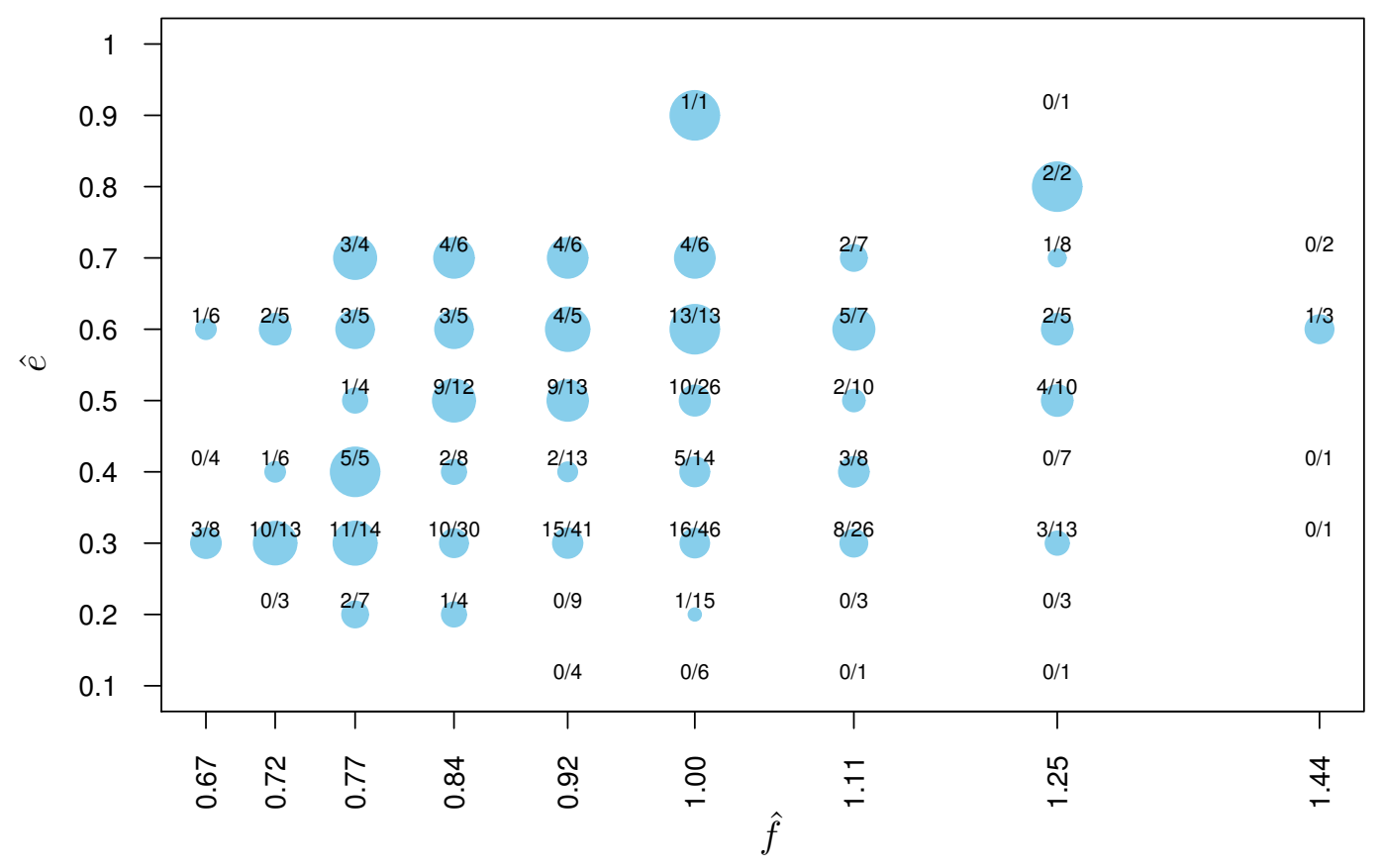

(a) Pig ex vivo defibrillation probabilities

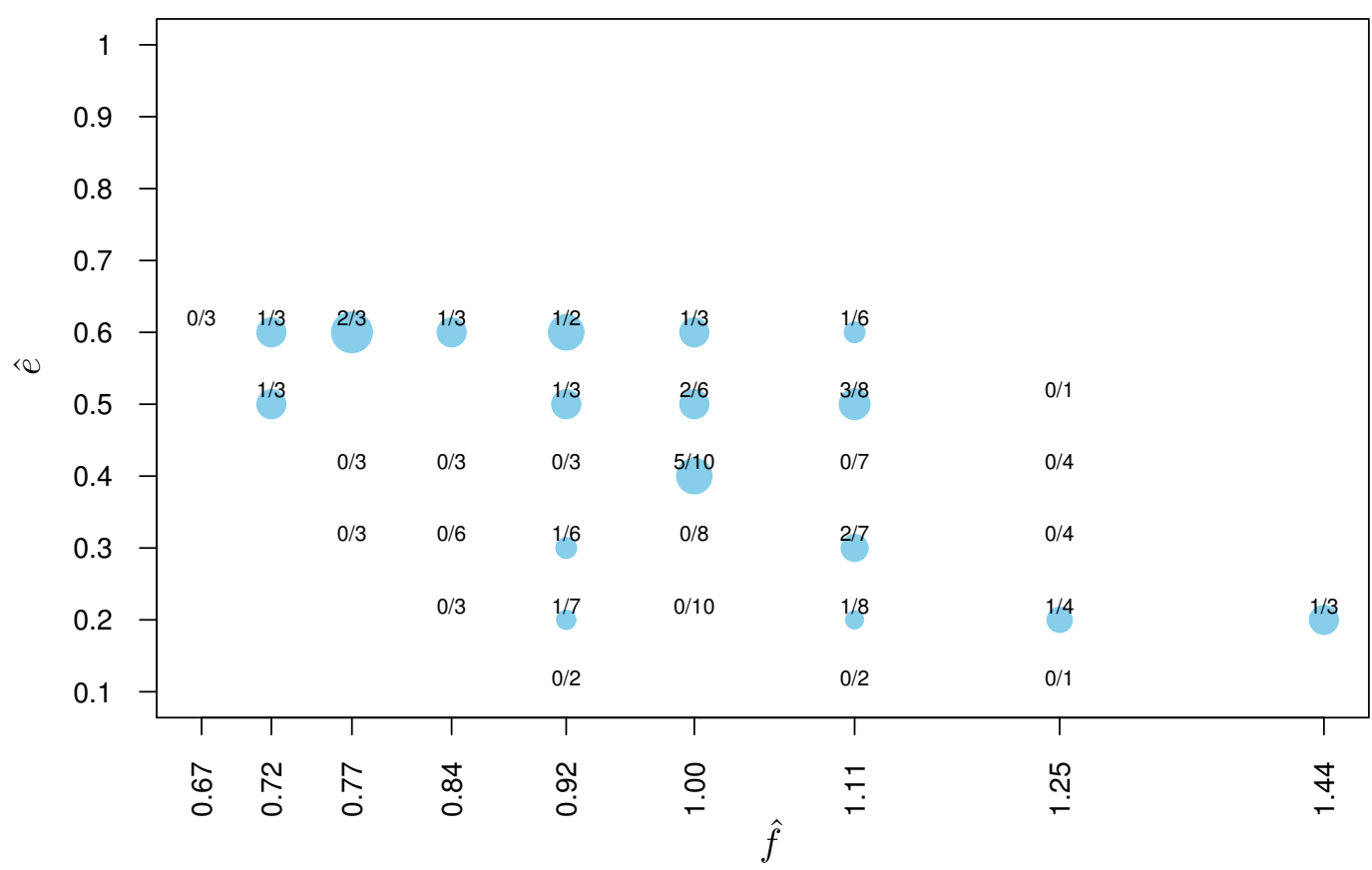

(b) Dog ex vivo defibrillation probabilities

FIGURE 8.17: Defibrillation probabilities for LEAP at different normalized frequencies $\hat{f}$ and energies $\hat{e}$. The area of the circles corresponds to the success ratio which is also given as numbers (success/total attempts) above the symbols. For frequency-energy configurations without numbers, no data is available. There is no clear bias for the dog experiments (bottom plot), but in pig hearts (top) the evaluation shows that $f_{\Omega \Omega}<f_{\circlearrowleft}$ is clearly more promising to terminate fibrillation. 


\subsubsection{Frequency Dependency of LEAP Success}

Owing to the higher overall number of defibrillation in ex vivo experiments, more data could be collected about LEAP at different frequency ratios. In analogy to figure 8.8 in the in vivo results section, figure 8.17 shows the defibrillation success rates for different energies and frequencies.

The number of successful defibrillations at lower energies in the dog experiments is not sufficient for conclusive results regarding the optimum pacing frequencies. The pig experiments, on the other hand, clearly show that underdrive LEAP $\left(f_{\Omega \Omega}<f_{\Im}\right)$ yield a significantly better success rate overdrive LEAP. A t-test comparing the success probabilities for each energy level showed that with $p<0.005$ lower frequencies result in higher defibrillation probabilities. This result confirmed the hints from the respective in vivo analysis (section 8.1.4). The success probabilities (pig experiments) are:

\begin{tabular}{r|rr}
$\hat{e}$ & $\operatorname{Pr}(\hat{f}<1)$ & $\operatorname{Pr}(\hat{f}>1)$ \\
\hline 0.5 & 0.65 & 0.30 \\
0.4 & 0.31 & 0.20 \\
0.3 & 0.47 & 0.28 \\
0.2 & 0.13 & 0.0
\end{tabular}

\subsubsection{Reliability of DFT Measurements}

Some ex vivo perfusion experiments suffer from similar variability in the single pulse DFT as in vivo experiments, complicating the interpretation of LEAP pulse energies. Prominent examples (see the energy plots in figure 8.9 to 8.14 for the measured energies) are:

\begin{tabular}{|c|c|c|}
\hline Experiment & DFT energies & Comment \\
\hline Pig, 2012-05-07 & $\begin{array}{l}\text { large variation in the single pulse DFT, } \\
\text { overlapping with LEAP energies }\end{array}$ & \\
\hline Pig, 2012-12-08 & $\begin{array}{l}\text { single pulse DFT varied from beyond the } \\
\text { amplifier's capacity }(\approx 50 \mathrm{~J}) \text { to about } 5 \mathrm{~J} .\end{array}$ & \\
\hline Dog, 2013-03-08 & $\begin{array}{l}\text { single pulse DFT declined nearly continu- } \\
\text { ally from } 0.8 \mathrm{~J} \text { to } 0.35 \mathrm{~J} \text {, interrupted by a } \\
\text { short and sharp drop after approximately } \\
110 \text { min after the start of the experiment. }\end{array}$ & $\begin{array}{l}\text { The same heart } \\
\text { showed a similar } \\
\text { drop in vivo be- } \\
\text { fore. }\end{array}$ \\
\hline
\end{tabular}

\footnotetext{
${ }^{4}$ In one experiment (2012-12-09) the actual single pulse energy was much higher than calculated here and beyond the technical limits of the amplifier during most parts of the experiment, certainly above $48 \mathrm{~J}$. On these grounds, the relative LEAP energy results were omitted for calculating the averages.
} 



\section{Chapter 9}

\section{Discussion}

\subsection{Prediction of Activation from Vessel Sizes}

I found that the diameter of the coronary vasculature of the heart follows a power law distributions, for atria $(\alpha=-2.74(5), \operatorname{dog})$ and ventricles $(\alpha=-2.75(30)$, dog $)$ and across different species, dogs and pigs $(\alpha=-3.47(76))$ (chapter 5). Although the exponent for pig hearts (the data is from the ventricles) is smaller than for dogs, this difference is not significant given the current data. My measured data differs significantly from the exponents which one can obtain from processing other published data by Kassab et al. [65] $(\alpha=-1.14(25))$. This deviation is likely due to the large variances in the published data.

The size distributions from dogs and pigs are in agreement with the hypothesis that the scaling of cardiac blood vessels is universal among different species. Yet differences between species are possible, so for a conclusive result, further measurements, both for pig hearts and possibly another species, are necessary in order to reliably evaluate their vascular scaling distributions.

Another power law, regarding the time until cardiac tissue was activated after externally applied electric fields, could be successfully linked with the size distributions. Taking into account the dimensionality of the tissue, we used a well-understood generic model of the behavior of excitable media to transform one power law into the other. The predicted activation time exponents were compatible with the measured values.

This connection clearly demonstrates that cardiac structure elements in the form of blood vessels constitute sources of wave emission. Wave emissions of this type are the probable agents for the efficacy of arrhythmia termination via LEAP and will be the key to understanding how arrhythmias can be terminated at low voltages. 


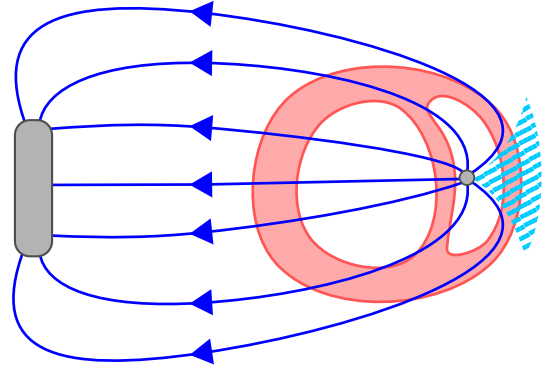

(a) ICD case and catheter

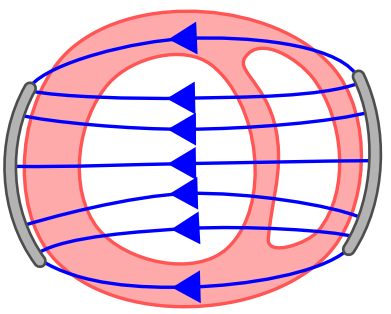

(b) patch electrodes

Figure 9.1: Schematic comparison of different electric field geometries. Figure a shows the field between the ICD case phantom on the left and the catheter electrode inside the right ventricle of the heart. The hatched area to the right marks the region with marginal or no electric field. (b) The electric field between two patch electrodes. Although it is weaker in the outer regions, there are no parts of the heart with vanishing field.

\subsection{Setup Evaluation}

The in vivo experimental setups provided reliable means to safely induce and terminate ventricular fibrillation in the animal, in configurations corresponding to ICDs in clinical practice and to previously published setups. The animals were typically physiologically stable for 2 to 5 hours, allowing about 40 defibrillation episodes. In all cases with sufficient oxygen supply, the heart could be used for another series of ex vivo experiments, which confirmed that the heart was not damaged substantially during thein vivo experiment.

The fact that the impedances and necessary single shock energies of the pig setup are comparable to those in human ICD patients implies that the demonstrated energy gains through LEAP can be expected not only in the animal model but also in human ICD patients. Insofar as the pig and dog experiments can be compared (tables on pp. 108 and 120 , it seems that patch electrodes are more beneficial for energy reduction through application of LEAP. One reason may possibly lie in the field geometry, patch electrodes prevent regions of zero electric field, compared to an intraventricular catheter and submuscular ICD can (figure 9.1).

The ex vivo setup continued to provide an adequate environment for the excised heart, keeping it active for several hours after initiation of the perfusion. The staining with voltage sensitive dyes not only allows contactless measurement of activation, it also indicates which parts of the heart are properly perfused. The energy requirements in the dog experiments were considerably lower than in the pig experiments, enabling the use of power amplifiers which could defibrillate the heart reliably at the common pulse widths. 


\begin{tabular}{lcc} 
Animal & in vivo & ex vivo \\
\hline Pig & $0.51(3)$ & $0.28(11)$ \\
Dog & $0.31(16)$ & $0.33(21)$
\end{tabular}

TABLE 9.1: Comparison of the minimum in required energy $\left(\frac{E_{n n \min }}{\left\langle E_{\Omega}\right\rangle}\right)$ with LEAP in the pig and dog heart experiments. The pig in vivo experiments were the only setup where the ICD case-catheter configuration was used routinely. In the other three cases, external electrodes were used.

This may be caused by the electrode placement directly on the epicardium, reducing the current which flows around the tissue and is therefore lost for wave emission.

Comparing the maximum achievable energy gains in vivo and ex vivo (see table 9.1, data from the previous chapter), one finds that the values in the dog experiments are nearly identical. The ex vivo energy gains in the pig experiments reproduced the dog results, whereas in vivo only about $50 \%$ energy gain could be obtained. This is another strong indication that the electric field geometry may play an important role for the efficiency of LEAP.

\subsubsection{Clinical Relevance}

With single pulse defibrillation energies typically between 10 and $20 \mathrm{~J}$, the pig setup quantitatively reproduces the energies of clinical ICD implants. Since also the impedances were in the range expected for ICDs, the setup with the intraventricular catheter and ICD case phantom seem to be a good model system for real ICD applications. The experimental defibrillator used in these experiments is built on the same principles as commercially available ICDs (charging of capacitors to the desired voltage, discharging with or without polarity switching at custom-chosen times), lowering the barrier between experimental basic research and application.

\subsection{Defibrillation Energy Measurements}

The most commonly used quantitative measure to compare the efficiency of defibrillation methods is the energy delivered to the subject. Of course the actual effect on the heart is mediated by the current [115] which on the length scale of cells flows across the cell membranes and thereby increases the membrane potential sufficiently to initiate an action potential. Thus the relation between the total energy as delivered by the defibrillation device and the defibrillation efficacy depends on many factors affecting the current density inside the cardiac tissue: geometry of heart, thorax and electrodes, impedance through the tissue and on the electrodes' surface, but also the waveform 
parameters of the defibrillating pulse. Nevertheless, measuring the delivered energy has been the de facto standard for comparing defibrillation efficiency [115] and, all other factors being constant, does not carry any serious disadvantages.

A major issue typically arises when measuring the defibrillation requirement, due to the stochastic nature of the fibrillation and defibrillation process. The error margin for clinical DFT measurements is, depending on the exact method, on the order of $50 \%$ the half-probability energy $E_{50}$. A "single measurement" in this context typically consists already of several fibrillation/defibrillation episodes. This means that with only few of these measurements, a quantitative efficiency comparison of several defibrillation methods is not possible.

\subsubsection{The Unequal-Pairs Method}

The Unequal-Pairs energy measurement method as described in section 8.0 (p. 100 ) and in the appendix takes into account all defibrillation attempts and therefore is suitable for initial estimation of the defibrillation energies and further confirmation or correction of this value. Thus, it can be seen as an enhancement of the midpoint DFT measurement [116] which starts with an educated guess of $E_{50}$ and then continues up or down, depending on the initial outcome, until the defibrillation success changes. If the energy is always varied so as to provoke a defibrillation outcome change (increasing upon failure and decreasing upon success), the Unequal-Pairs method's energy distribution has its maximum at $E_{50}$ for many tries.

Especially in the in vivo experiments, this DFT measurement method produced consistent and quick energy checks which allowed to quickly detect fluctuations in the required energy or confirm that the energy remained stable.

It should be noted however, that the deduced defibrillation "threshold" still depends on the protocol, although less so than other methods. Even more protocol independent methods would be, for example, maximum likelihood estimations (MLE) of the dose response curve. An MLE would always result in the best dose response curve, although its error margins of course still would depend on the test energies.

\subsubsection{DFT drift}

The biggest obstacle for reliable measurements of energy ratios in the experiment was the sometimes seemingly erratic behavior of the DFT. 
The literature on the behavior of DFT over the course of prolonged fibrillation and defibrillation experiments is inconclusive, although a number of surveys have been conducted in this area. Babbs et al. [117. found a drop of only about $10 \%$ in the defibrillation threshold energy during experiments of up to 8 hours duration. In contrast, Kerber et al. [118] identified two major factors leading to a substantial decrease in the DFT: duration of experiment (a decline in the DFT of $22 \%$ during 2 hours) and hypoxia (30\% decline if the $\mathrm{pO}_{2}$ was reduced to $40 \mathrm{mmHg}$ ). In [119], a DFT drop of about 30 to $40 \%$ seems to take place over $90 \mathrm{~min}$ (six DFT measurements consisting of several defibrillation episodes each).

In contrast to these findings, in our experiments we saw sudden jumps in the DFT which could not be adequately explained by slow drifts. Whether the ischemic periods or other physiological factors such as the recovery duration after fibrillation episodes are responsible and how to control the defibrillation susceptibility remains a question to be answered in future experiments. The general DFT drifts experienced e.g. in the ex vivo experiments on 2012-06-11 (figure 8.10) and 2013-03-08 (figure 8.14) however seem to confirm the previous findings in [118] and [119].

\subsection{Energy Reduction with LEAP}

A major objective of the LEAP experiments was to compare the efficiency of LEAP in clinically more realistic setups to previous results published in [45]. The reported energy reduction by $90 \%$ in ex vivo ventricular fibrillation experiments suggested in vivo verification in an animal model and possibly in man studies as the next steps.

In all experiments with a largely homogeneous field (all ex vivo and the dog in vivo experiments), we found that the single pulse defibrillation energy could be reduced by approximately $70 \%$ (table 9.1), independently of species or the exact electrode configuration. While this reduction does not reach the energy gain from [45, it is substantial and could be confirmed in a large number of experiments. The energy reduction in the pig in vivo experiments only reached $50 \%$, a result that may well have been caused by zones of comparatively weak electric fields (see figure 9.1).

Presumably the main difference between the experimental setup used for the results reported in [45] and our configuration was the preparation of the heart before the experiment. While we used whole hearts in nearly all cases, the previous ventricular LEAP experiments were conducted on wedge preparations of at most about $10 \mathrm{~cm}$ diameter. This difference leads to a number of implications: 
- As Panfilov convincingly argued in [99], the size of the substrate may play a crucial role in determining the complexity of the supported dynamics. Generally the dynamic behavior of smaller media is less complex, therefore fibrillation in wedge preparations may be easier to terminate by lower energies.

- An essentially flat portion of myocardium may provide less possibilities for reentry than a system of two fully connected ventricles which additionally still includes the Purkinje fiber system.

- The exact mechanism of LEAP is not fully understood yet, but one plausible hypothesis is the unpinning of pinned excitation waves from heterogeneities. In the wedge preparations, such an unpinned wave would hit the (comparatively close) boundary much more easily than in an intact heart. If on the other hand, the tissue boundaries are too far away to be hit by the spiral core immediately, the spiral may reattach to the heterogeneity again.

\subsection{Frequency Dependency of LEAP Success}

The pacing frequency $f_{\Omega \text { 几 }}$ of LEAP is maybe the most important adjustable parameter to decrease the defibrillation energy. Even if there is a clear reference frequency $f_{\odot}$ describing the dominant fibrillation behavior, the choice of $f_{\Omega \Omega}$ is not trivial.

Since free spirals may be driven by pacing with frequencies faster than their own [120], $f_{\text {几л }}>f_{\circledast}$ may seem plausible at first. For pinned excitation spirals - if they play an important role in VF - the situation is more complex though. Depending on the orientation of the spiral relative to the electric field, the wave emitted by a LEAP pulse from the pinning center may detach the spiral. Intuitively, many pulses at $f_{\Omega} \neq$ $f_{\odot}$ should hit the spiral at different phases, thereby increasing the chance of hitting the unpinning window $\rho_{\mathrm{uw}}$ [48. Behrend et al. [49] showed however that for some models, if $f_{\text {几л }}>f_{\odot}$, the pulses may lock the spiral into a phase where unpinning is impossible, whereas slower pacing frequencies may scan the phase until they finally find the unpinning window.

One question behind the experiments was which pacing frequencies optimized the required pacing energies. Our in vivo (section 8.1.4) and ex vivo (section 8.2.4) experiments showed a clear preference for $f_{\Omega}<f_{\odot}$, although there was not sufficient data to obtain a specific optimum frequency ratio.

This result suggests that the unpinning mechanism from sources close to or directly at vortex cores plays an important role in LEAP and that successive entrainment of the cardiac tissue as proposed in [45] on its own is not sufficient to terminate VF. 


\section{Chapter 10}

\section{Conclusion and Outlook}

\subsection{Structure and Function}

We have clearly demonstrated experimentally the existence of a link between cardiac tissue structure and susceptibility to low-energy pulses. This result is not only a confirmation of predictions from theory and cell culture experiments. In the bulk tissue of healthy hearts, the cardiac vasculature provides the necessary structural heterogeneities, but diseased hearts may also possess other structural features, such as scars, fibrotic or remodeled tissue. The experiments suggest that the tissue response to electric stimuli is characteristic for the embedded heterogeneities.

Therefore, our results may open the path for new diagnostic methods and individually optimized treatments which take into account patient specific characteristics. This may lower the energy requirements of gentle defibrillation methods such as LEAP even further.

To date, we experimentally only investigated the effects of the vasculature. Other heterogeneities such as fibrotic tissue and their interaction with low-energy electric stimulation remains to be modeled theoretically and examined in experiments.

\subsection{LEAP Energy}

We successfully demonstrated the efficacy of LEAP for terminating otherwise fatal ventricular fibrillation in a number of diverse experiments: for two species (pig and $\operatorname{dog}$ ), we performed LEAP on alive anesthetized animals and on isolated intact hearts in a Langendorff perfusion setup. LEAP was effective in all tested electric field geometries: 
nearly homogeneous fields and highly diverging fields modeled after clinically relevant defibrillation catheter settings.

In all experiments with a reasonably homogeneous electric field distribution, I showed that termination of VF with LEAP is consistently possible with up to $70 \%$ energy reduction, compared to the standard single pulse defibrillation. On the other hand, substantially less energy reduction was achieved in those experiments with highly inhomogeneous fields. I confirmed that underdrive pacing is beneficial for LEAP success, which supports the hypothesis that a major factor for LEAP is the direct access to vortex cores of fibrillatory activity (as outlined in [45]) and which will be of practical importance for the further development of optimized pacing waveform.

In order to decrease the energy requirement even more and to increase the success rates for low energies, more theoretical and numerical work about the underlying mechanisms is required as well as refinement of the pacing protocol. The combination of different approaches to terminate fibrillation like the unpinning of vortices (with underdrive) and gradual synchronization of tissue (with overdrive pacing), or real-time decision systems for the onset timing of LEAP are promising candidates for further research topics.

\subsection{LEAP Experiments}

For the in vivo and ex vivo LEAP trials, we built an experiment framework consisting of a multitude of components, with minimal differences between the experiments. Highenergy defibrillation devices, recording of physiological data and customizable software were shared between in vivo and ex vivo setups, for dog and pig experiments. Ex vivo, the hearts were kept in physiological conditions by a mostly custom-built Langendorff perfusion setup which allowed the additional acquirement of optical voltage mapping signals.

The LEAP experiments provided reliable electrical and electrophysiological data for hours, irrespective of species, electrode setup or if the experiment was in vivo or ex vivo. We found some evidence that increased resting times between fibrillation episodes in vivo may be beneficial for long-term stability of defibrillation energy requirements, although for reliable results more data is required.

In order to further optimize the success rates of LEAP, a reasonable improvement would be an automatic control framework capable of e.g. synchronization of LEAP with activation patterns [121] or automatic adjustment of pacing frequencies. 
We found significantly better LEAP energy gains for more homogeneous electric field geometries, so an important question for the future is if and under which conditions the clinical catheter-can configuration can be used efficiently for LEAP. A major obstacle for the use of epicardial patch electrodes seems to be the increased perioperative mortality as compared to intraventricular catheter electrodes [122]. Here additional research is certainly necessary in order to find the optimal electrode configuration. To further increase the completeness of electro-optical data gathered from ex vivo experiments, endoscopic optical mapping of the interventricular septum would be a promising opportunity. 



\section{Appendix A}

\section{Derivations}

\section{A.1 Solution to the Bidomain Boundary Problem at Cir- cular Boundaries}

As was first pointed out by Pumir and Krinsky [47, external fields at boundaries of an excitable medium described by the monodomain equations modify the membrane potential as follows:

We start with the linearized ( $\left.\phi_{\mathrm{m}}=\phi_{\text {resting }}+\phi \approx \phi_{\text {resting }}\right)$ monodomain and boundary condition equations (4.4) and (4.5) as introduced in section 4.1.1.

$$
\begin{aligned}
\Delta \phi-\frac{1}{\lambda^{2}} \phi & =0 \\
\mathbf{n} \cdot(\nabla \phi-\mathbf{E}) & =0
\end{aligned}
$$

In this representation, the "electrotonic space constant" $\lambda$ is constructed from the intraand extracellular specific conductivities, the surface to volume ratio and the effective transmembrane conductivity $G_{\mathrm{m}}$ (assuming $\left.j_{\text {ion }}\left(\phi_{\mathrm{m}}\right) \approx G_{\mathrm{m}} \phi\right)$ :

$$
\lambda=\sqrt{\frac{1}{\frac{1}{\sigma_{\mathrm{e}}}+\frac{1}{\sigma_{\mathrm{i}}}} \frac{1}{\chi G_{\mathrm{m}}}}
$$

Let $E=|\mathbf{E}|$ and let $\theta_{0}$ be the field's angular direction. Then for a circular obstacle (i.e. an obstacle representing a circular boundary) of radius $R, 4.5$ can be expressed in polar coordinates $(r, \theta)$ :

$$
\partial_{r} \phi-E \cos \left(\theta-\theta_{0}\right)=0
$$


According to this representation, $\phi(r, \theta)$ can be factorized into $\phi(r) \cdot \cos \left(\theta-\theta_{0}\right)$. The following expression for $\phi(r, \theta)$ follows this factorization and solves (4.4) [47]:

$$
\phi(r, \theta)=-\lambda E \frac{K_{1}\left(\frac{r}{\lambda}\right)}{K_{1}^{\prime}\left(\frac{R}{\lambda}\right)} \cos \left(\theta-\theta_{0}\right)
$$

Here, $K_{1}$ is the modified Bessel function of the second kind. Since this function decreases strictly monotonically for increasing $r$, the change in membrane potential $\phi(r, \theta)$ is largest directly on the boundary, i.e. at $r=R$. The normalized membrane potential change (or depolarization) is plotted against $\frac{\lambda}{R}$ in figure 4.2 in the main text.

The physically relevant question is: How does the depolarization depend on the obstacle size and is it sufficient to initialize an action potential, i.e. does it reach a critical threshold value? For this, it is sufficient to consider $\frac{\phi}{\lambda E}$ on the boundary in the case of $\theta=\theta_{0}+\pi$. Let $R^{\prime}=\frac{R}{\lambda}$, then:

$$
\begin{aligned}
\phi\left(R, \theta_{0}+\pi\right) & =\lambda E \frac{K_{1}\left(R^{\prime}\right)}{K_{1}^{\prime}\left(R^{\prime}\right)} \\
& =\lambda E \frac{2 K_{1}\left(R^{\prime}\right)}{-K_{0}\left(R^{\prime}\right)-K_{2}\left(R^{\prime}\right)} \\
& \approx \lambda E R^{\prime} \text { for } R^{\prime} \ll 1 \\
& =E \cdot R
\end{aligned}
$$

This implies for the minimum size of an obstacle in order to depolarize tissue above a certain threshold:

$$
R_{\min } \propto \frac{1}{E}
$$

As Bittihn et al. [50] showed, maximum depolarization is not reached for obstacles of infinite size, as one might expect from figure 4.2 , but for boundaries with negative curvatures or negative $R$. For this case of negative curvature, basically $K_{1}$ replaced by $I_{1}$, the modified Bessel function of the first kind.

Boundaries with negative curvature, i.e. concave shape, correspond to regions of excitable medium which are enclosed by purely extracellular space. One conclusion drawn in [50] is that e.g. a circular spot of excitable medium will have its maximum depolarization at a radius of several $\lambda$. 


\section{A.2 Defibrillation threshold estimation}

\section{A.2.1 Maximum Likelihood Estimation of a Defibrillation Probability}

The most accurate way to characterize the defibrillation dose response curve is fitting a parametrized curve to the data. Since the dose response curve only describes probabilities, the method of maximum likelihood estimation is appropriate in this case.

Consider the probability Pr that a defibrillation attempt is successful (we denote this with the success variable $s$ which shall become $s=1$ for successful defibrillation and 0 otherwise).

Now for a given pulse energy $E$ this probability shall be described by the probability function $F_{\boldsymbol{\alpha}}$ which is parametrized by a set of parameters $\boldsymbol{\alpha}$ :

$$
\operatorname{Pr}(E) \equiv \operatorname{Pr}(s=1 \mid E)=F_{\boldsymbol{\alpha}}(E)
$$

Now consider a set of measurements

$$
\boldsymbol{X}=\left\{x_{1}, x_{2}, \ldots\right\}=\left\{\left(E_{1}, s_{1}\right),\left(E_{2}, s_{2}\right), \ldots \mid\left(E_{i}, s_{i}\right) \in\left(\mathbb{R}_{0}^{+} \times\{0,1\}\right)\right\}
$$

and

$$
\begin{aligned}
\boldsymbol{E} & =\left\{E_{1}, E_{2}, \ldots\right\} \\
\boldsymbol{S} & =\left\{s_{1}, s_{2}, \ldots\right\}
\end{aligned}
$$

where each measurement $x_{i}$ consists of an input (the energy $E_{i}$ ) and an outcome (the success $s_{i}$ ). The defibrillation probability function can be deduced from the answer to the following question: Given $\boldsymbol{E}$, for which set of parameters $\boldsymbol{\alpha}$ is the outcome $\boldsymbol{S}$ least unlikely? This is equivalent to the following formulation:

\section{Problem A.1 (MLE for defibrillation)}

Find $\boldsymbol{\alpha}$ so that $\operatorname{Pr}(\overline{\boldsymbol{S}} \mid \boldsymbol{E})=\prod_{i}\left|F_{\boldsymbol{\alpha}}\left(E_{i}\right)-s_{i}\right|$ becomes minimal.

Since $\operatorname{Pr}(\overline{\boldsymbol{S}} \mid \boldsymbol{E})$ can be easily calculated for any $\boldsymbol{\alpha}$, this maximum likelihood estimation (MLE) problem can quickly be solved by standard numerical methods if the measurements $\boldsymbol{X}$ contain sufficient information. 


\section{A.2.2 Error Estimation of the Defibrillation Probability}

The error of the estimated parameters $\boldsymbol{\alpha}$ can be estimated from the logarithmic improbability $L$ :

$$
L(\overline{\boldsymbol{S}}, \boldsymbol{E})_{\boldsymbol{\alpha}}=\log (\operatorname{Pr}(\overline{\boldsymbol{S}} \mid \boldsymbol{E}))=\sum_{i} \log \left(\left|F_{\boldsymbol{\alpha}}\left(E_{i}\right)-s_{i}\right|\right)
$$

The inverse of the information matrix $\boldsymbol{I}(\hat{\boldsymbol{\alpha}})$ [123, p. 265f.] gives an estimate for the standard errors $\delta_{i}$ of the estimated parameters $\hat{\boldsymbol{\alpha}}$ :

$$
\begin{aligned}
\boldsymbol{I}(\boldsymbol{\alpha}) & =-\frac{\partial^{2} L(\overline{\boldsymbol{S}}, \boldsymbol{E}, \boldsymbol{\alpha})}{\partial \boldsymbol{\alpha} \partial \boldsymbol{\alpha}^{T}} \\
\delta_{i} & =\sqrt{\boldsymbol{I}(\hat{\boldsymbol{\alpha}})_{i i}^{-1}}
\end{aligned}
$$

Unfortunately the number of measurements required to keep this error low is too high for practical use in in vivo experiments where the actual values $\boldsymbol{\alpha}$ may drift on the timescale of a few dozen measurements.

\section{A.2.3 The "Unequal Pairs" Method}

The "Unequal Pairs" method invented for this work analyzes pairs of measurements in time. Consider a series $\boldsymbol{X}$ of events as defined in A.5 A.7 above. One way to gain information about the dose response function $F_{\boldsymbol{\alpha}}(E)$ is to look exclusively at those events whose neighbor event produced a different outcome, i.e. those $i$ where $s_{i} \neq s_{i+1}$. We shall consider such a pair a measurement in the context of this method since we only use this pair's energy for further calculations. For the sake of brevity, let us define

$$
\begin{aligned}
\operatorname{Pr}(x \mid E) & \equiv \operatorname{Pr}(s=x \mid E) \text { for } x \in\{0,1\} \\
\operatorname{Pr}(E) & \equiv \operatorname{Pr}(1 \mid E) .
\end{aligned}
$$

Then the measurement probability $\operatorname{Pr}($ measurement $\mid E) \equiv P_{M}(E)$ that the success of two events differs is simply given by:

$$
P_{M}(E)=\operatorname{Pr}(01 \vee 10 \mid E)=\operatorname{Pr}(01 \mid E)+\operatorname{Pr}(10 \mid E)=2 \operatorname{Pr}(10 \mid E)=2 \operatorname{Pr}(E)(1-\operatorname{Pr}(E))
$$




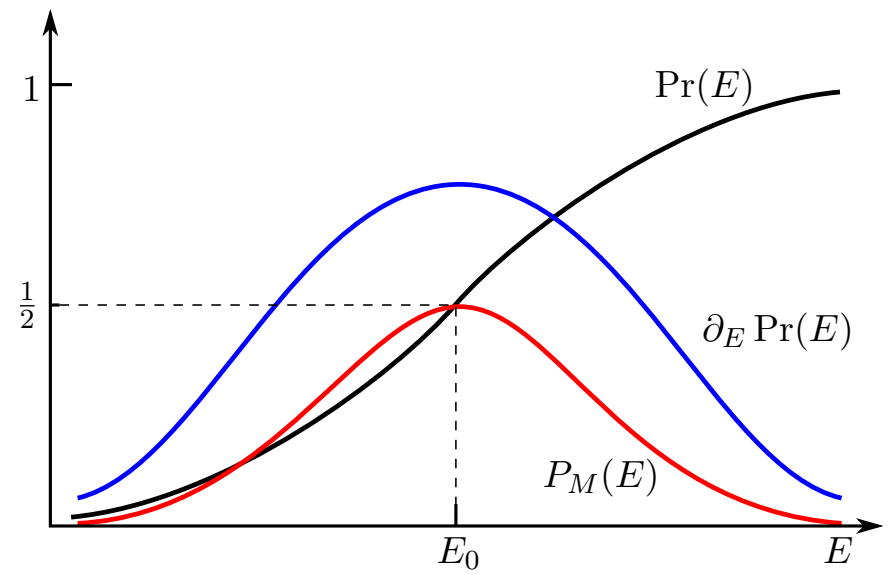

FIgURE A.1: Sketched probability functions for success and measured energies, for an ("anti-symmetric") $\operatorname{Pr}(E)$ which is anti-symmetric around $E_{0}$. Note that $\operatorname{Pr}\left(E_{0}\right)=$ $\frac{1}{2}=P_{M}\left(E_{0}\right)=\max _{E} P_{M}(E)$, which holds for any strictly monotonic $\operatorname{Pr}(E)$ (with a conveniently chosen $E_{0}$ ).

This leads to the immediate conclusion concerning its derivatives:

$$
\begin{aligned}
\partial_{E} P_{M}(E) & =2\left((1-\operatorname{Pr}(E)) \partial_{E} \operatorname{Pr}(E)-\operatorname{Pr}(E) \partial_{E} \operatorname{Pr}(E)\right) \\
& =2(1-2 \operatorname{Pr}(E)) \partial_{E} \operatorname{Pr}(E) \\
\Rightarrow \partial_{E} P_{M}(E) & =0 \text { for } \operatorname{Pr}(E)=\frac{1}{2}
\end{aligned}
$$

We see that the maximum of $P_{M}(E)$ has a value of $\frac{1}{2}$ and is located at the energy where $\operatorname{Pr}(E)=\frac{1}{2}$ (for strictly monotonic $\operatorname{Pr}(E)$ ). Additionally if $\operatorname{Pr}(E)$ is anti-symmetric (up to a constant shift) around a specific $E_{0}$, i.e. if for $E^{\prime} \equiv E-E_{0}, \operatorname{Pr}\left(-E^{\prime}\right)=1-\operatorname{Pr}\left(E^{\prime}\right)$, then

$$
P_{M}\left(E^{\prime}\right)=2 \operatorname{Pr}\left(E^{\prime}\right)\left(1-\operatorname{Pr}\left(E^{\prime}\right)\right)=2 \operatorname{Pr}\left(E^{\prime}\right) \operatorname{Pr}\left(-E^{\prime}\right)
$$

and therefore

$$
P_{M}\left(E^{\prime}\right)=P_{M}\left(-E^{\prime}\right) \Rightarrow P_{M} \text { is symmetric around } E_{0} .
$$

See also figure A.1 for an illustration of these relations.

\section{A.2.3.1 Special Case: The Logistic Function}

A common choice for $\operatorname{Pr}(E)$ is one of the most basic sigmoid functions, the logistic function $\operatorname{Pr}(E)=1 /\left(1-e^{-\left(\frac{E}{\sigma^{2}}-E_{0}\right)}\right)$. Apart from being straightforward to calculate, it has the convenient property of being the solution to the following equation, thereby 


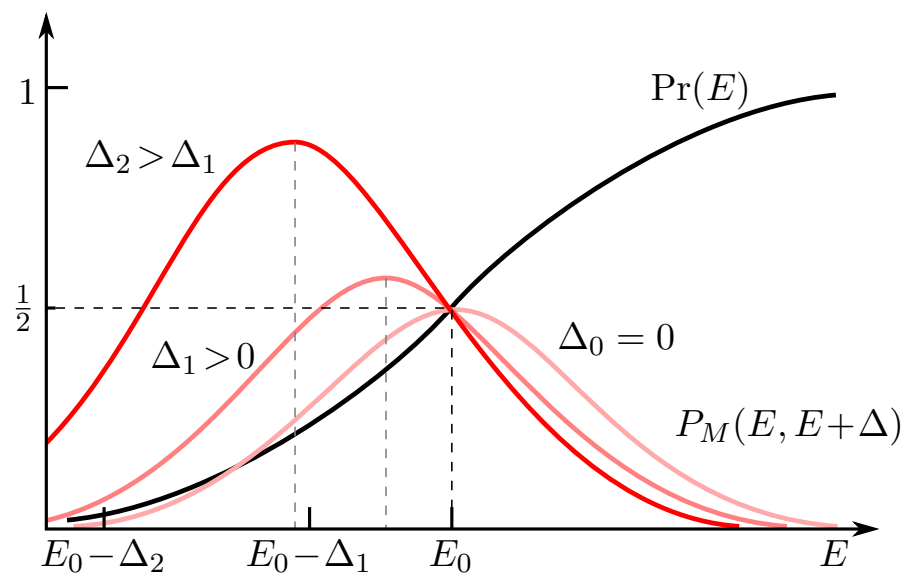

Figure A.2: Schematic of the measurement probabilities for several $\Delta$. Note how $P_{M}\left(E_{0}, E_{0}+\Delta\right)=\operatorname{Pr}\left(E_{0}\right)=\frac{1}{2}$ still holds for all $\Delta$.

closely linking the shapes of $P_{M}$ and Pr:

$$
P_{M}(E)=2 \sigma^{2} \frac{\mathrm{d}}{\mathrm{d} E} \operatorname{Pr}(E)
$$

Specifically, the measurement probability $P_{M}$ for this case is given by

$$
P_{M}(E)=\frac{2 e^{\frac{E}{\sigma^{2}}-E_{0}}}{\left(1+e^{\frac{E}{\sigma^{2}}-E_{0}}\right)^{2}}
$$

\section{A.2.3.2 Different Energies}

If the energies of the two neighboring events are different $(E$ and $E+\Delta)$, this is reflected on the measurement probability:

$$
\begin{aligned}
P_{M}(E, E+\Delta) & =\operatorname{Pr}(10 \mid E, E+\Delta)+\operatorname{Pr}(01 \mid E, E+\Delta) \\
& =\operatorname{Pr}(E)+\operatorname{Pr}(E+\Delta)-2 \operatorname{Pr}(E) \operatorname{Pr}(E+\Delta)
\end{aligned}
$$

If such a pair of events at $E$ and $E+\Delta, \Delta>0$, is interpreted as the measurement of an energy $E$ (as a reasonable lower bound), the previously established statement still holds:

$$
\operatorname{Pr}\left(E_{0}\right)=\frac{1}{2} \Rightarrow P_{M}\left(E_{0}, E_{0}+\Delta\right)=\operatorname{Pr}\left(E_{0}\right)=\frac{1}{2}
$$

The maximum of $P_{M}(E, E+\Delta)$ however is shifted compared to $\Delta=0$, again for strictly monotonically increasing $\operatorname{Pr}(E)$ we obtain:

$$
\begin{aligned}
& \frac{\mathrm{d}}{\mathrm{d} E} P_{M}(E, E+\Delta)= \\
& \operatorname{Pr}^{\prime}(E)+\operatorname{Pr}^{\prime}(E+\Delta)-2 \operatorname{Pr}^{\prime}(E) \operatorname{Pr}(E+\Delta)-2 \operatorname{Pr}(E) \operatorname{Pr}^{\prime}(E+\Delta)
\end{aligned}
$$


using $\operatorname{Pr}\left(E_{0}\right)=\frac{1}{2}$ :

$$
\begin{aligned}
& \begin{aligned}
P_{M}^{\prime}\left(E_{0}, E_{0}+\Delta\right) & =\operatorname{Pr}^{\prime}\left(E_{0}\right)+\operatorname{Pr}^{\prime}\left(E_{0}+\Delta\right)-2 \operatorname{Pr}^{\prime}\left(E_{0}\right) \operatorname{Pr}\left(E_{0}+\Delta\right)-\operatorname{Pr}^{\prime}\left(E_{0}+\Delta\right) \\
& =\operatorname{Pr}^{\prime}\left(E_{0}\right)\left(1-2 \operatorname{Pr}\left(E_{0}+\Delta\right)\right)<0
\end{aligned} \\
& P_{M}^{\prime}\left(E_{0}-\Delta, E_{0}\right)=\operatorname{Pr}^{\prime}\left(E_{0}\right)\left(1-2 \operatorname{Pr}\left(E_{0}-\Delta\right)\right)>0
\end{aligned}
$$

This means that the maximum of $P_{M}(E, E+\Delta)$ is located somewhere between $E_{0}-\Delta$ and $E_{0}$, as shown schematically in figure A.2.

Therefore the interpretation of experimentally measured results with the Unequal Pairs method must take into account the difference in energies of neighboring events, measurement protocols with smaller energy differences are less prone to these systematic errors. 



\section{Appendix B}

\section{Supplementary Material}

\section{B.1 Vascular Size Distributions}

\section{B.1.1 Dog Hearts}

Listed in this section are the vascular size distributions of the dog heart preparations (ventricles and atria). The results of the corresponding power law fits are listed in table $5.1 \mathrm{a}$ and $5.1 \mathrm{~b}$ in chapter 5 . These plots were first published in [45].

\section{Dog Atria}

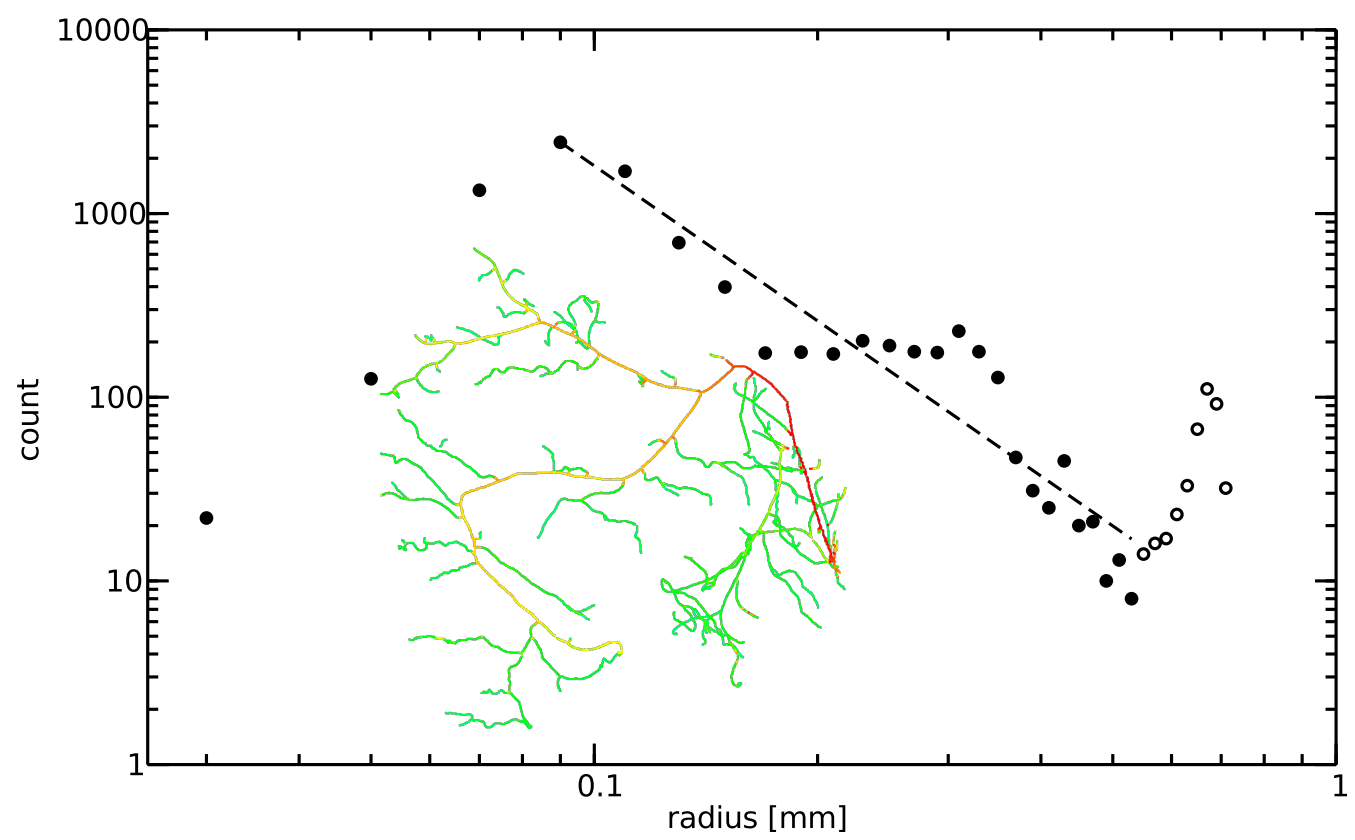

Figure B.1: Vascular size distributions in dog atria: Preparation \#1. 


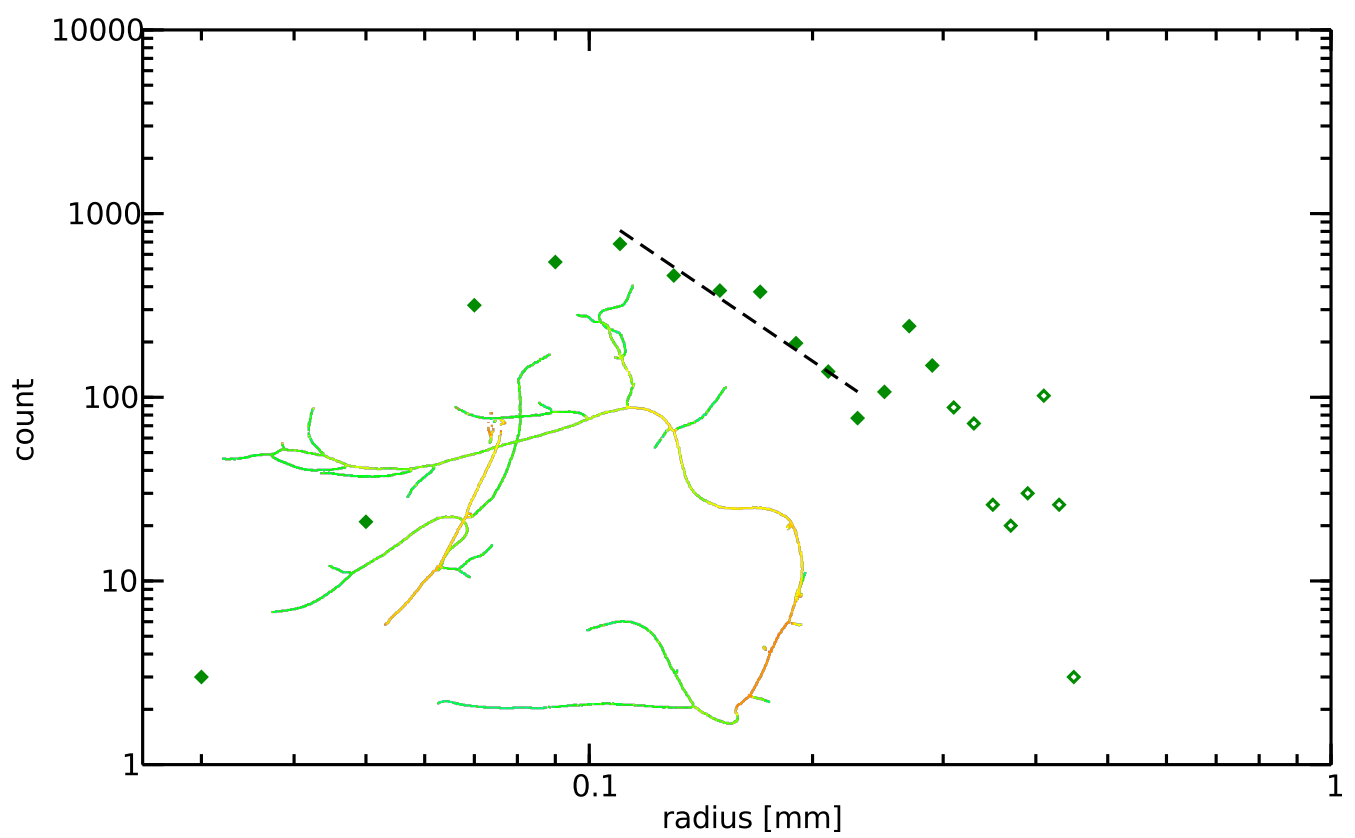

FiguRE B.2: Vascular size distributions in dog atria: Preparation \#2.

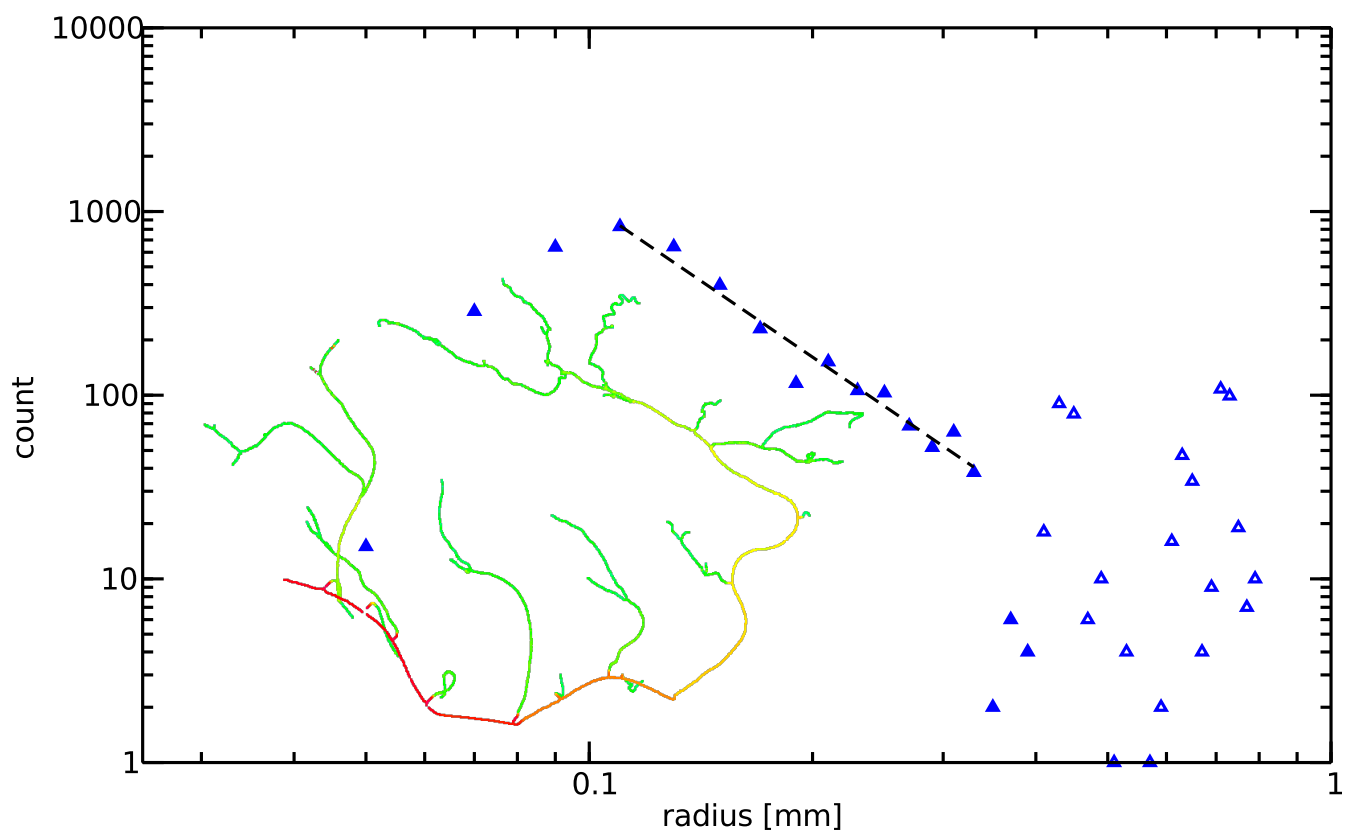

Figure B.3: Vascular size distributions in dog atria: Preparation \#3. 


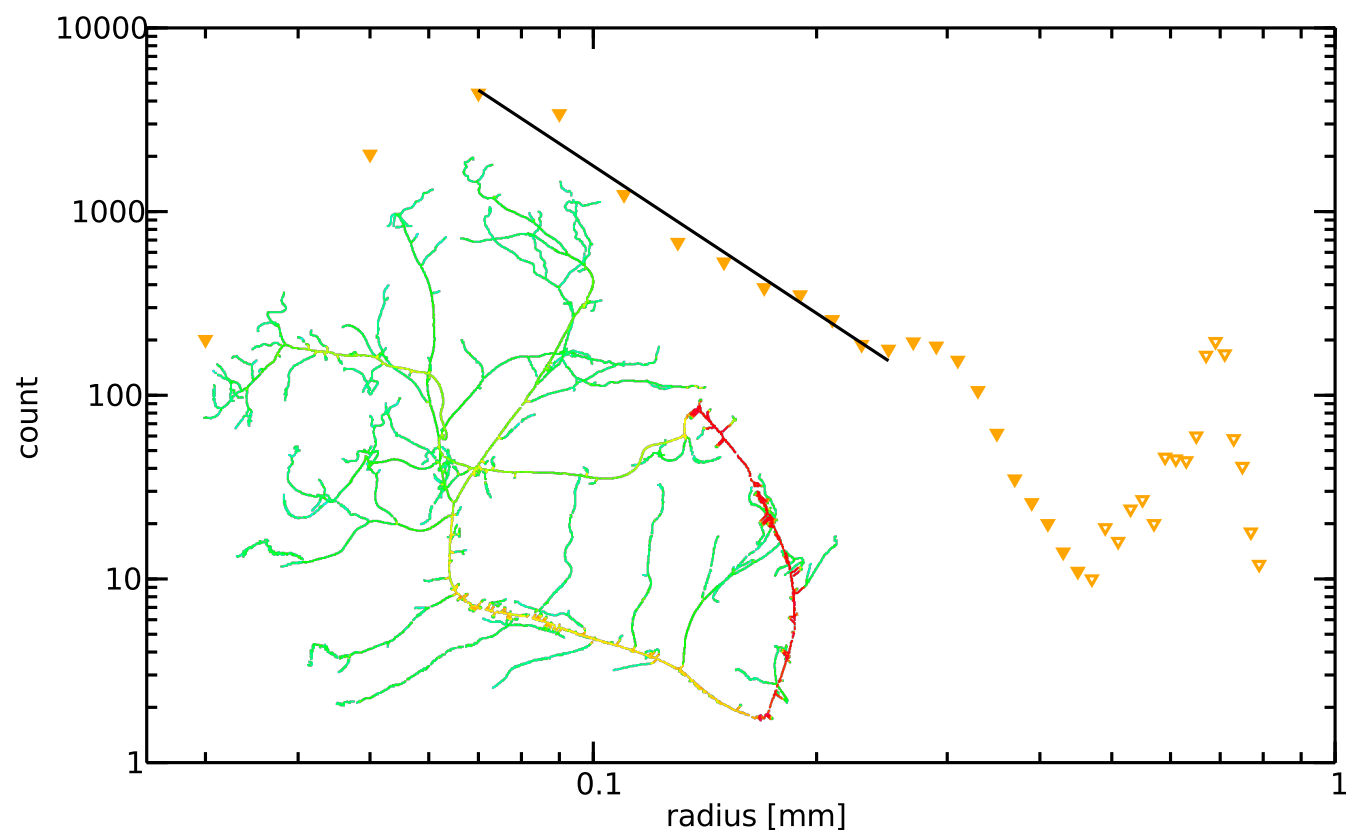

Figure B.4: Vascular size distributions in dog atria: Preparation \#4.

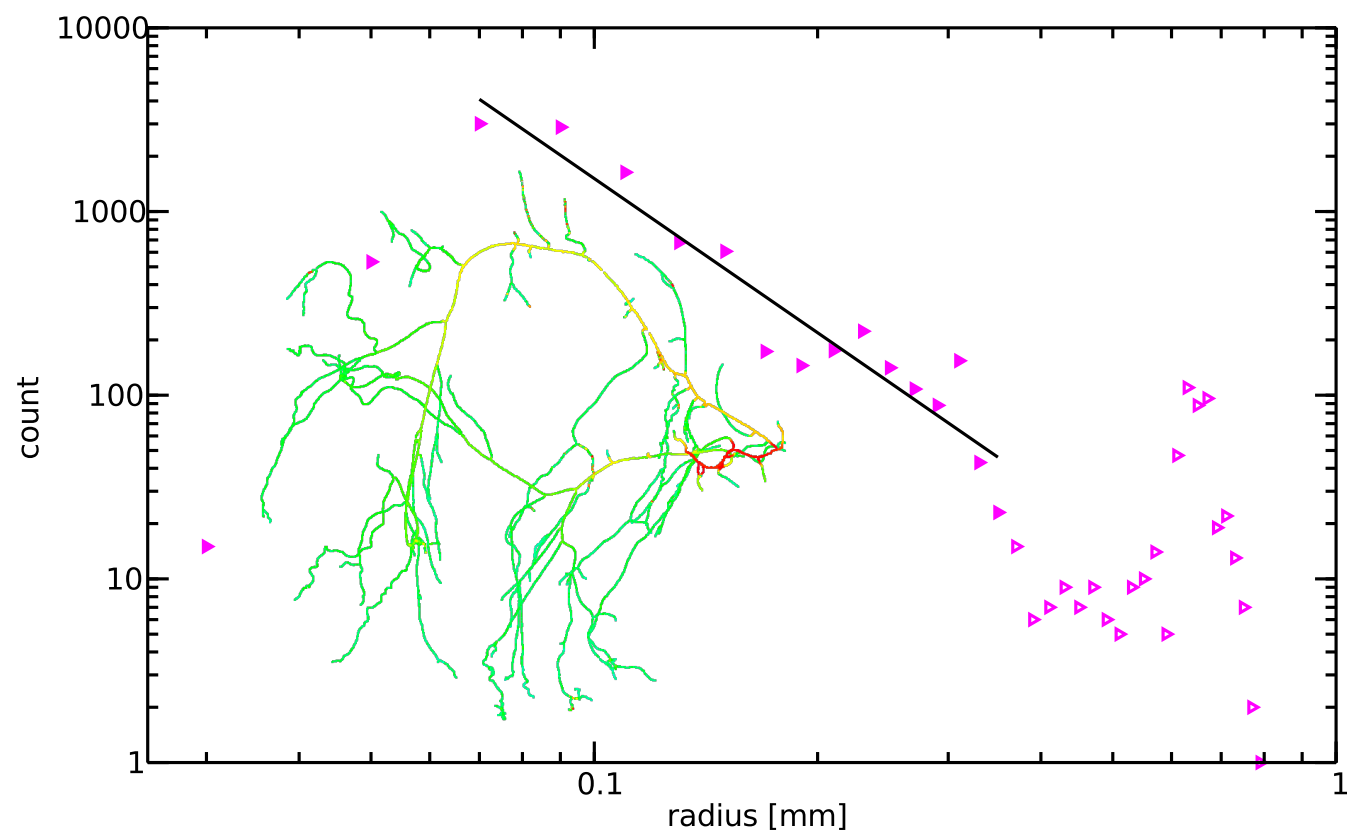

Figure B.5: Vascular size distributions in dog atria: Preparation \#5. 


\section{Dog Ventricles}

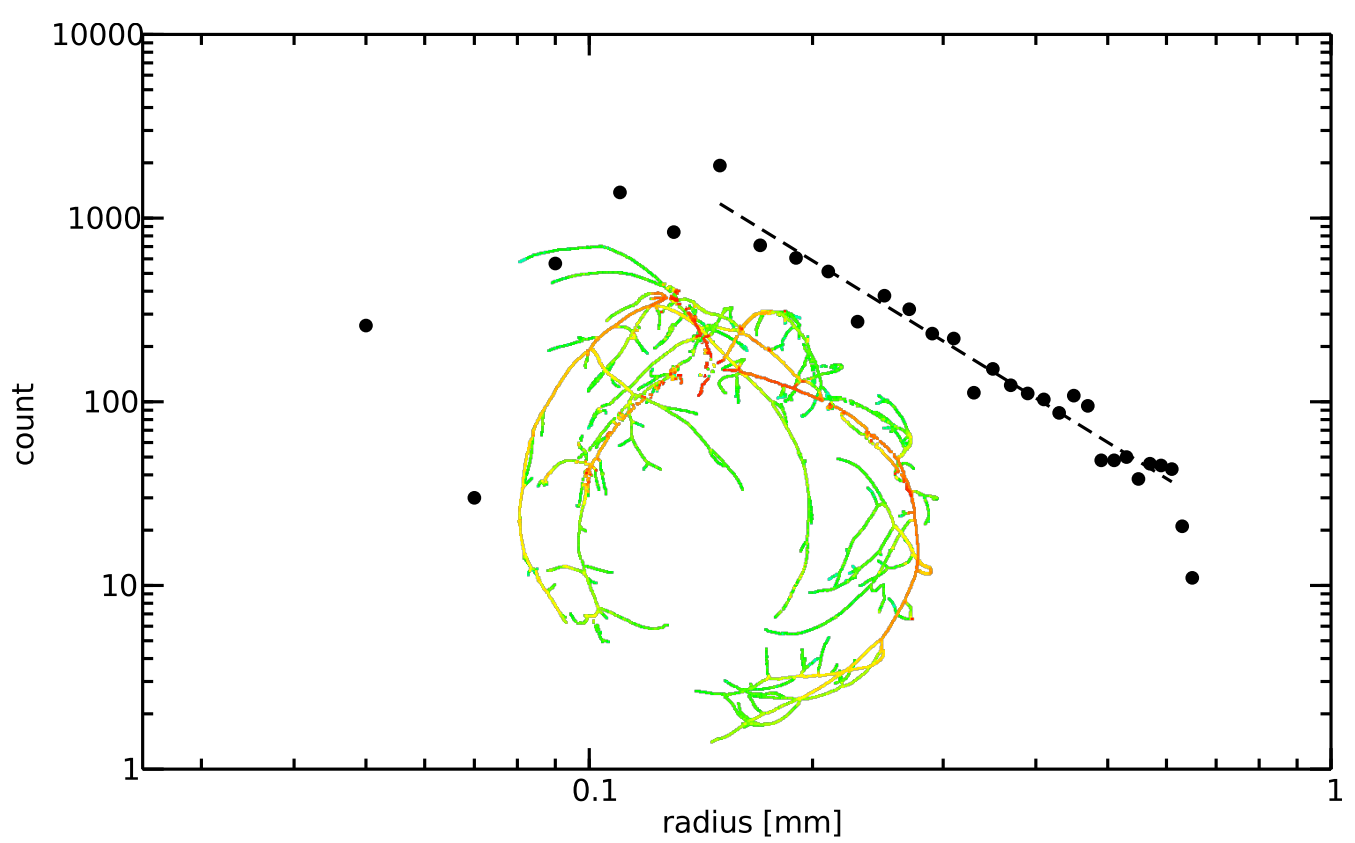

Figure B.6: Vascular size distributions in dog ventricles: Preparation \#1.

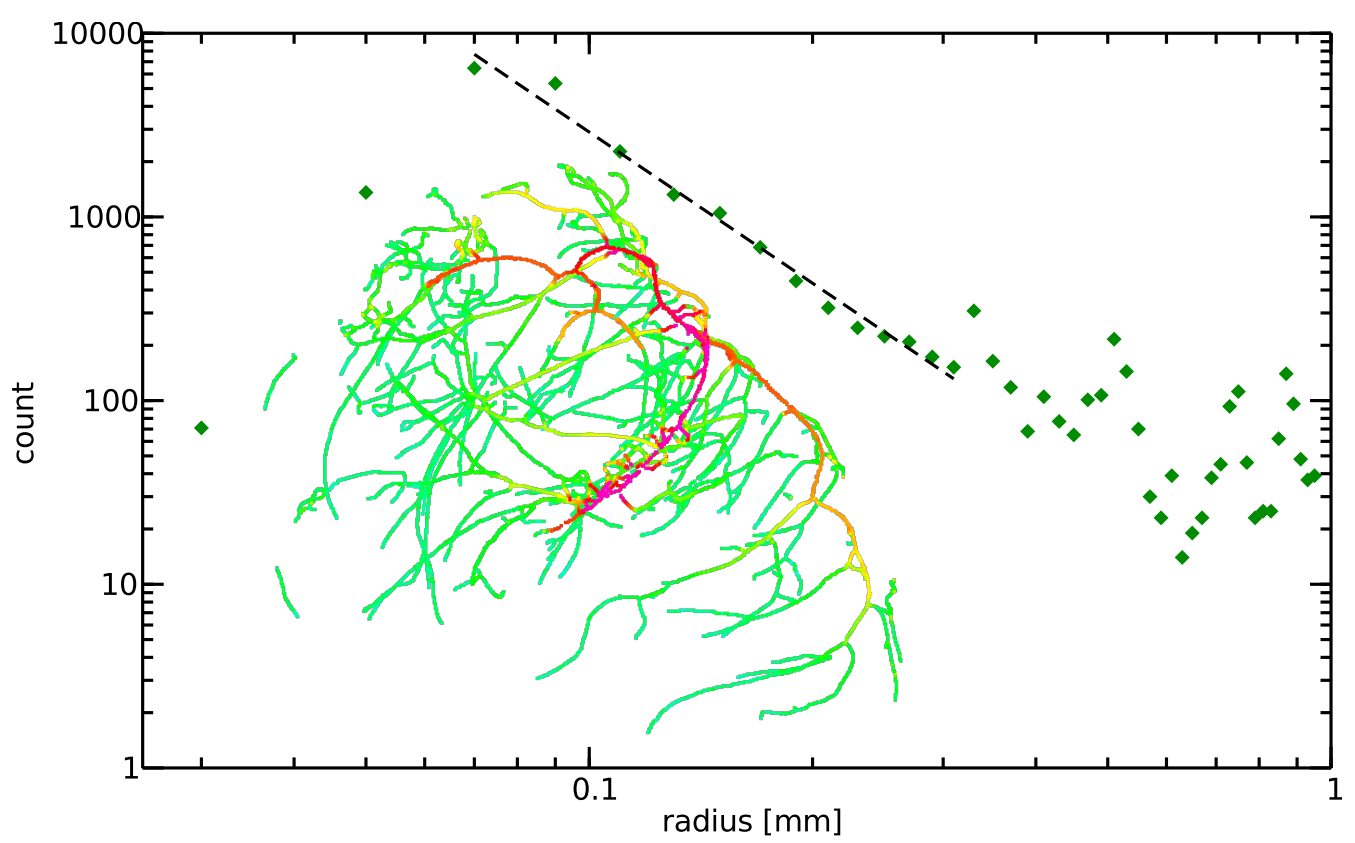

Figure B.7: Vascular size distributions in dog ventricles: Preparation \#2. 


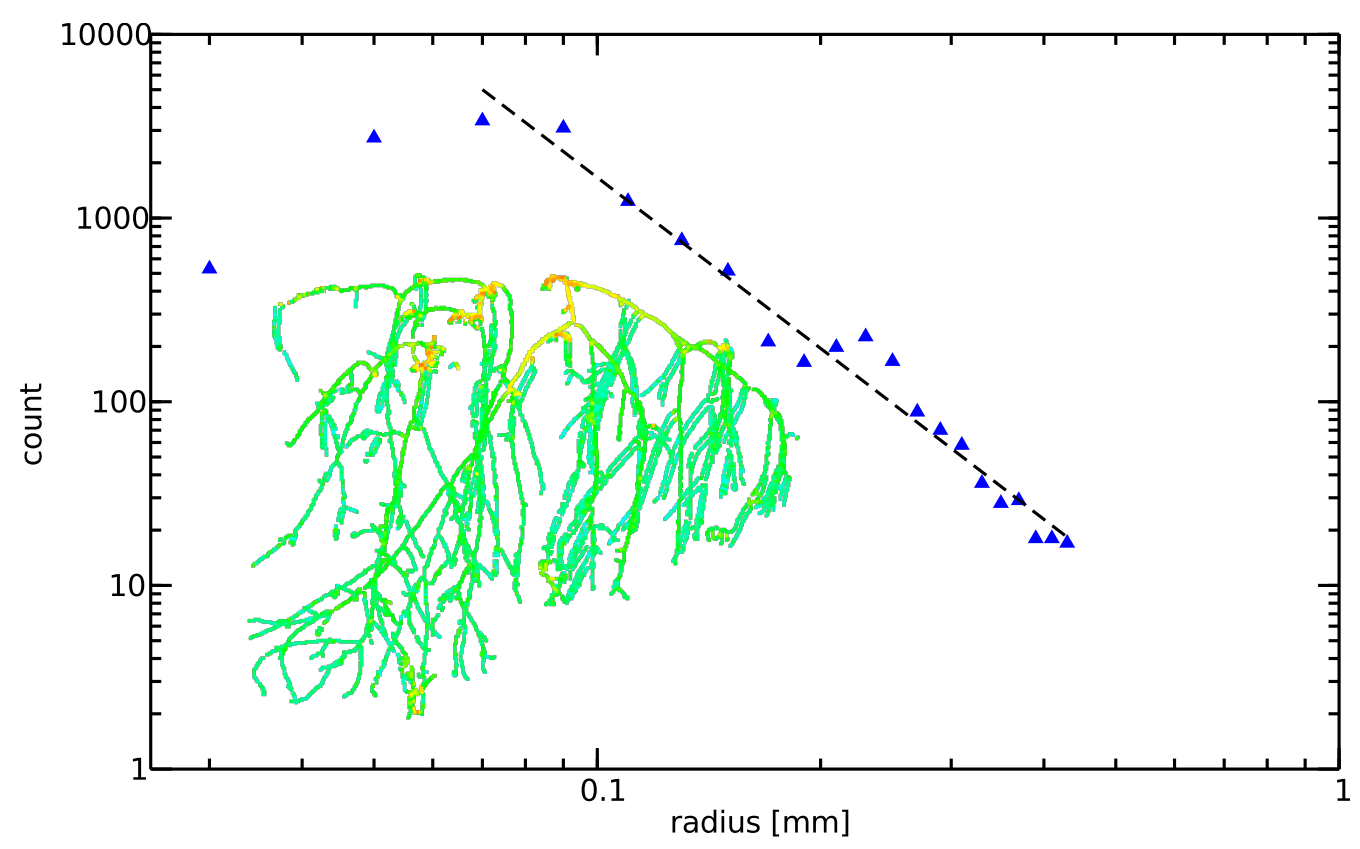

FiguRe B.8: Vascular size distributions in dog ventricles: Preparation \#3. 


\section{B.1.2 Pig Hearts}

Listed in this section are the vascular size distributions of the pig heart preparations (ventricular vasculature). The resulting of the exponents $\alpha$ of the corresponding power law fits are listed in section 5.1 .2 in the chapter Heterogeneity Characterization Results

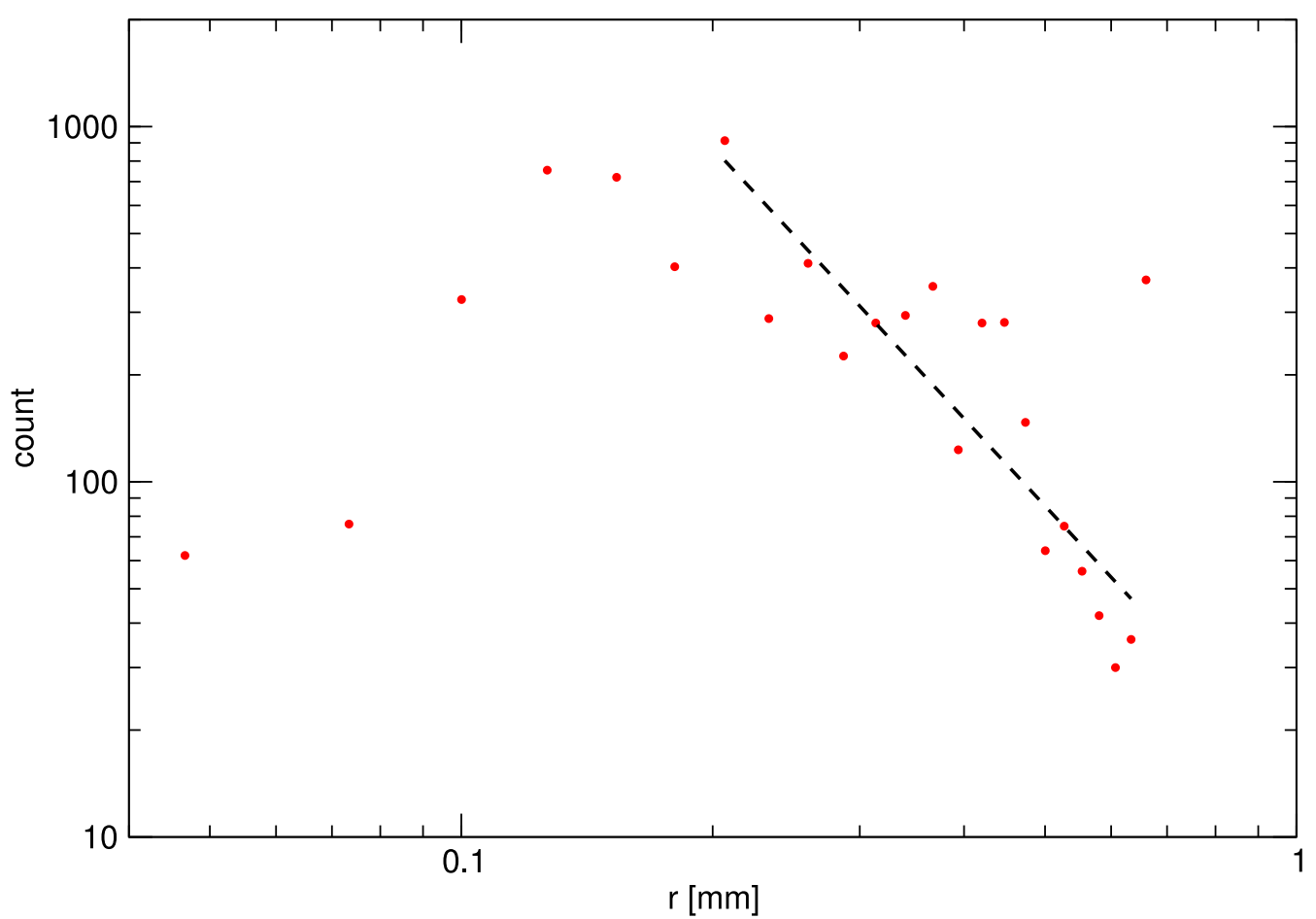

Figure B.9: Vascular size distributions in porcine ventricles: Preparation \#1. The rendering has been lost. 


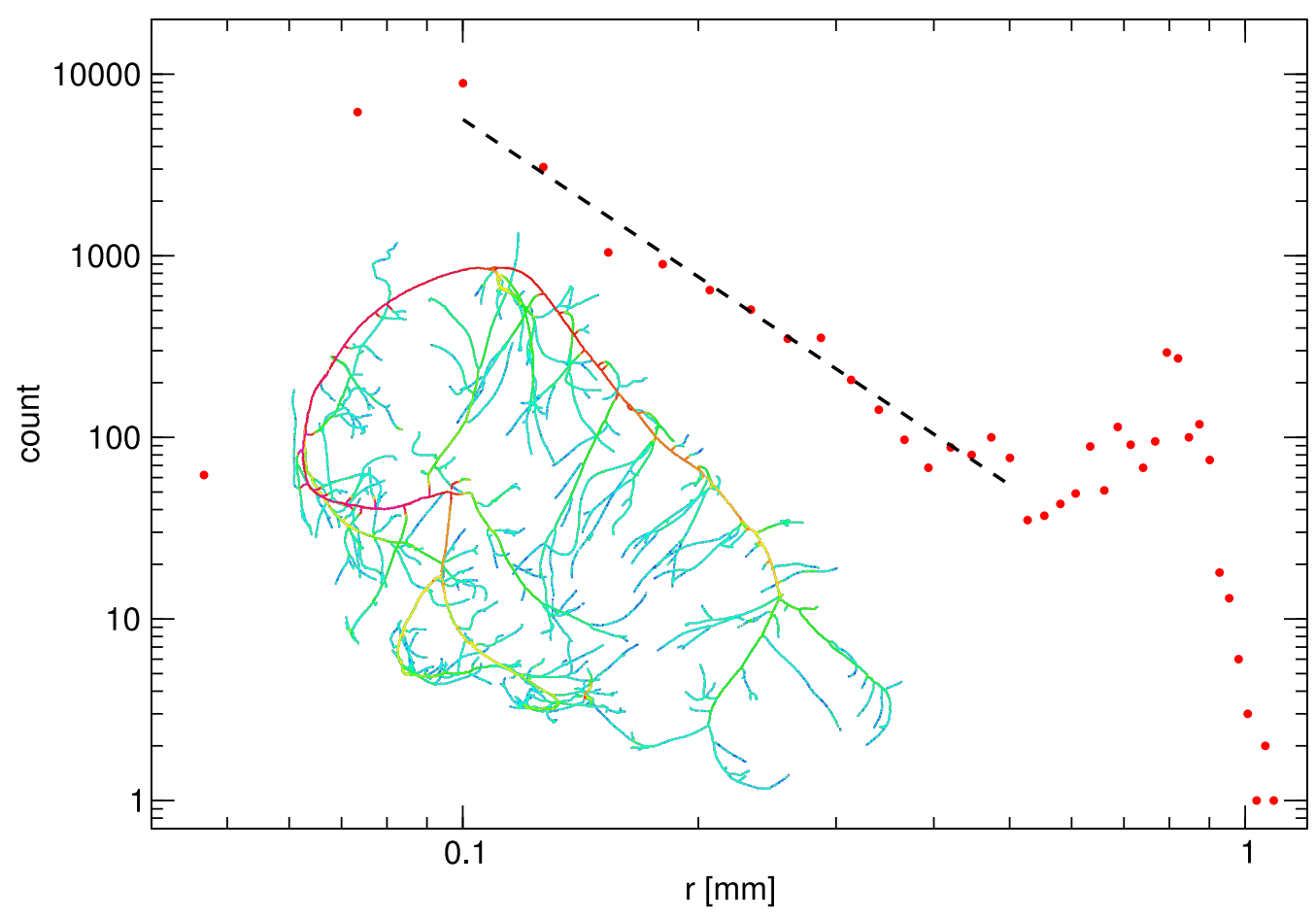

FiguRE B.10: Vascular size distributions in porcine ventricles: Preparation \#2.

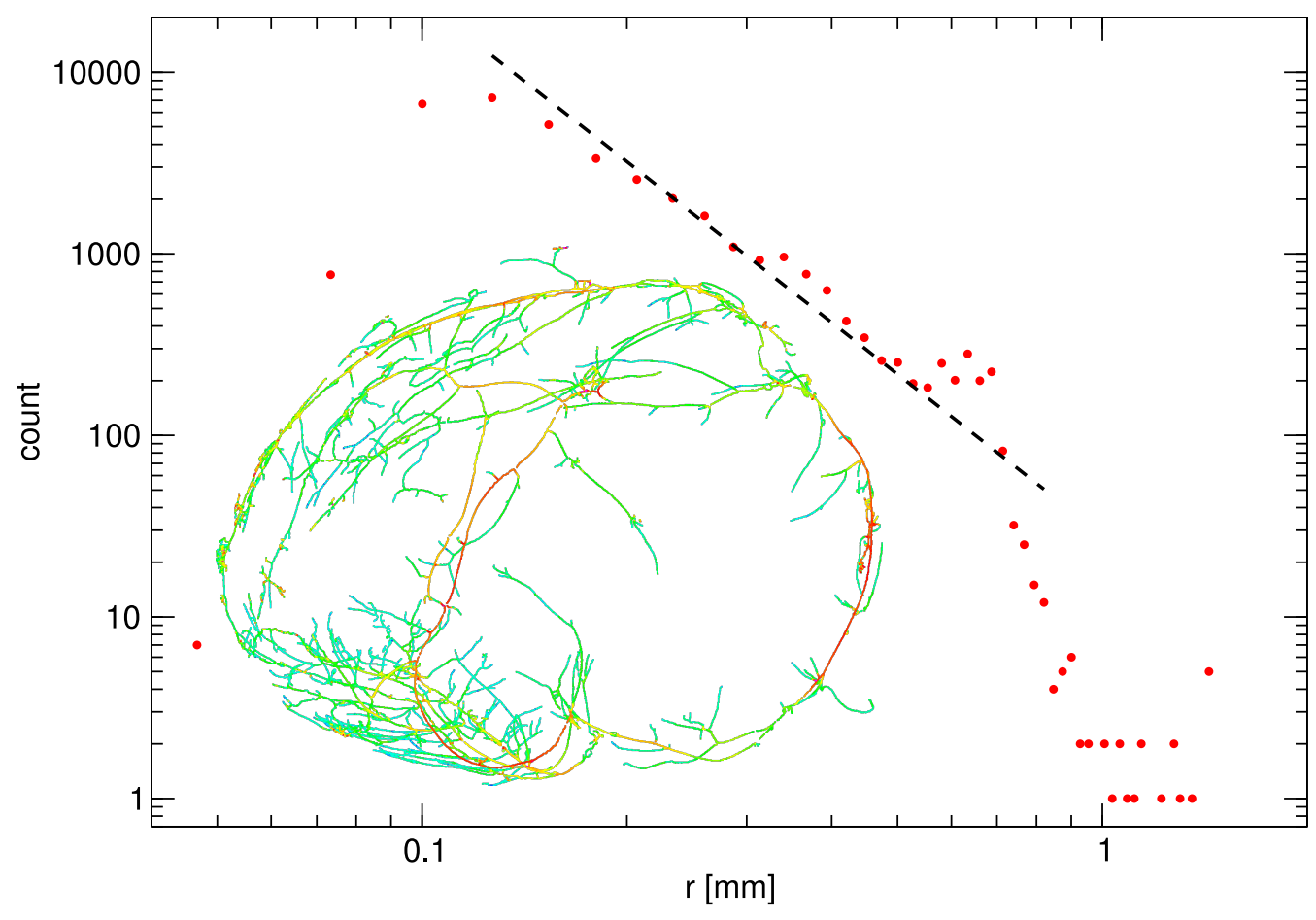

Figure B.11: Vascular size distributions in porcine ventricles: Preparation \#3. 


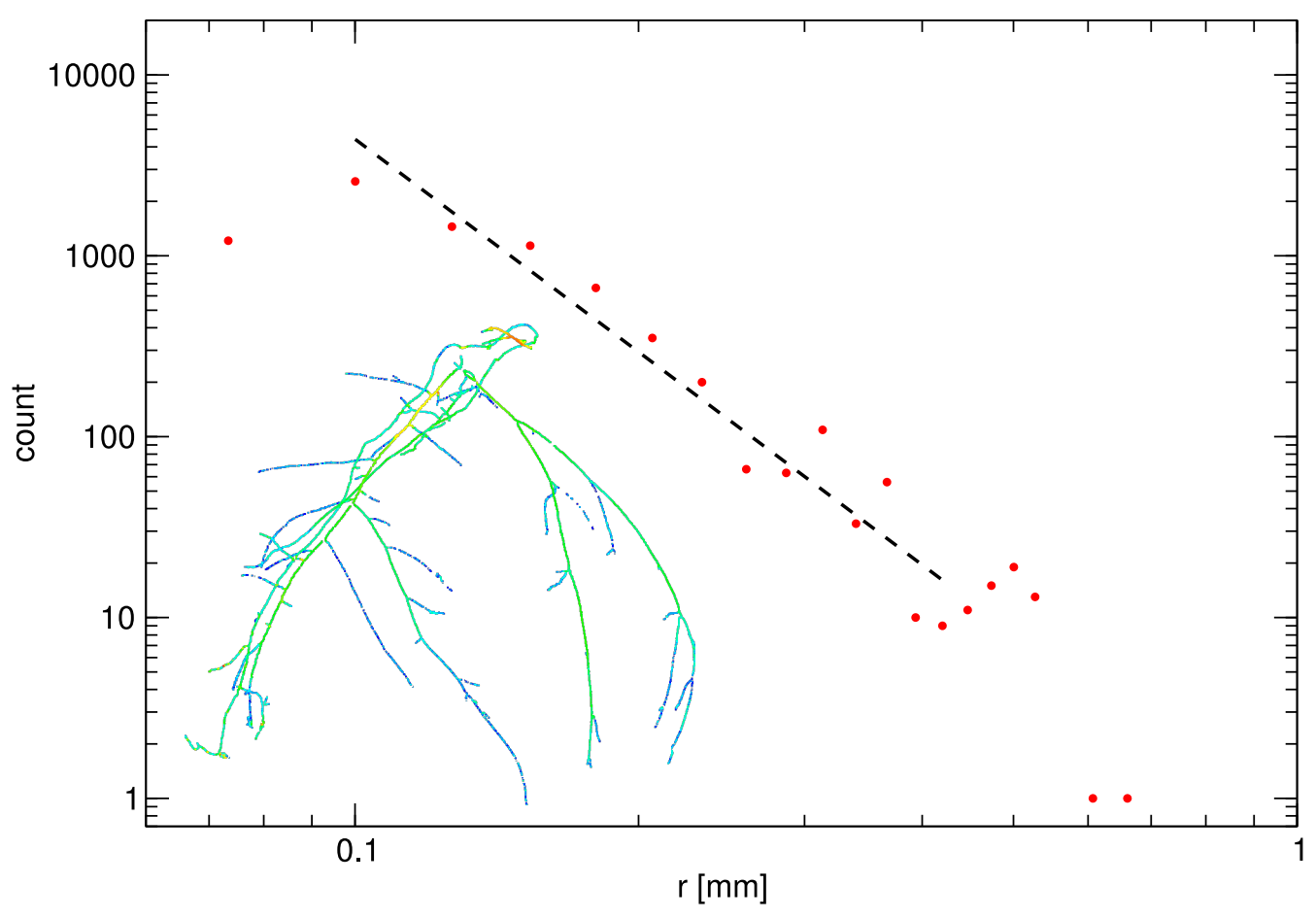

FiguRE B.12: Vascular size distributions in porcine ventricles: Preparation \#4.

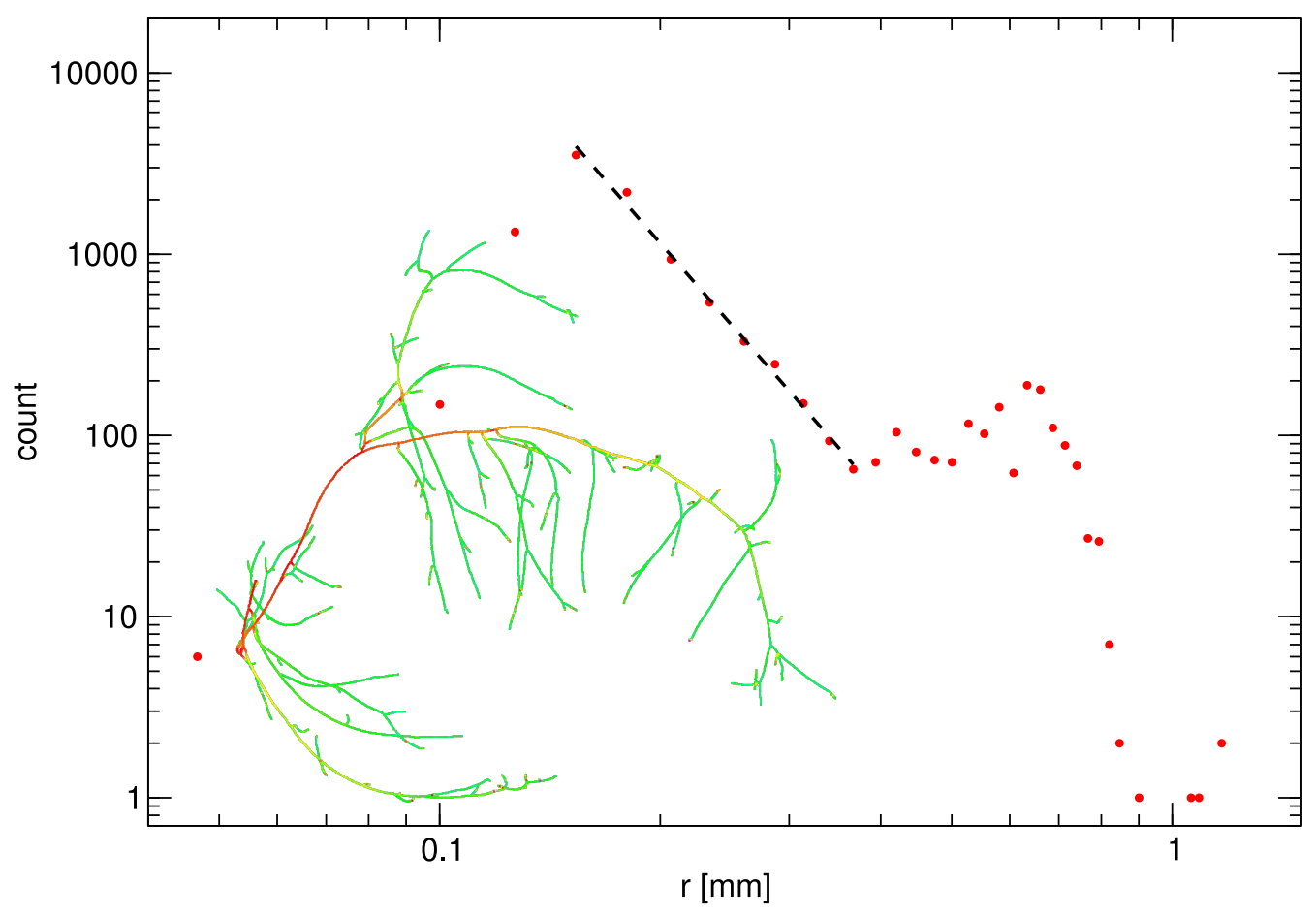

FiguRE B.13: Vascular size distributions in porcine ventricles: Preparation \#5. 


\section{B.2 Frequency Dependency of LEAP Success}

The following plots show the frequency dependency for each in vivo and ex vivo experiment. Only on few experiments there was enough data to draw meaningful conclusions.
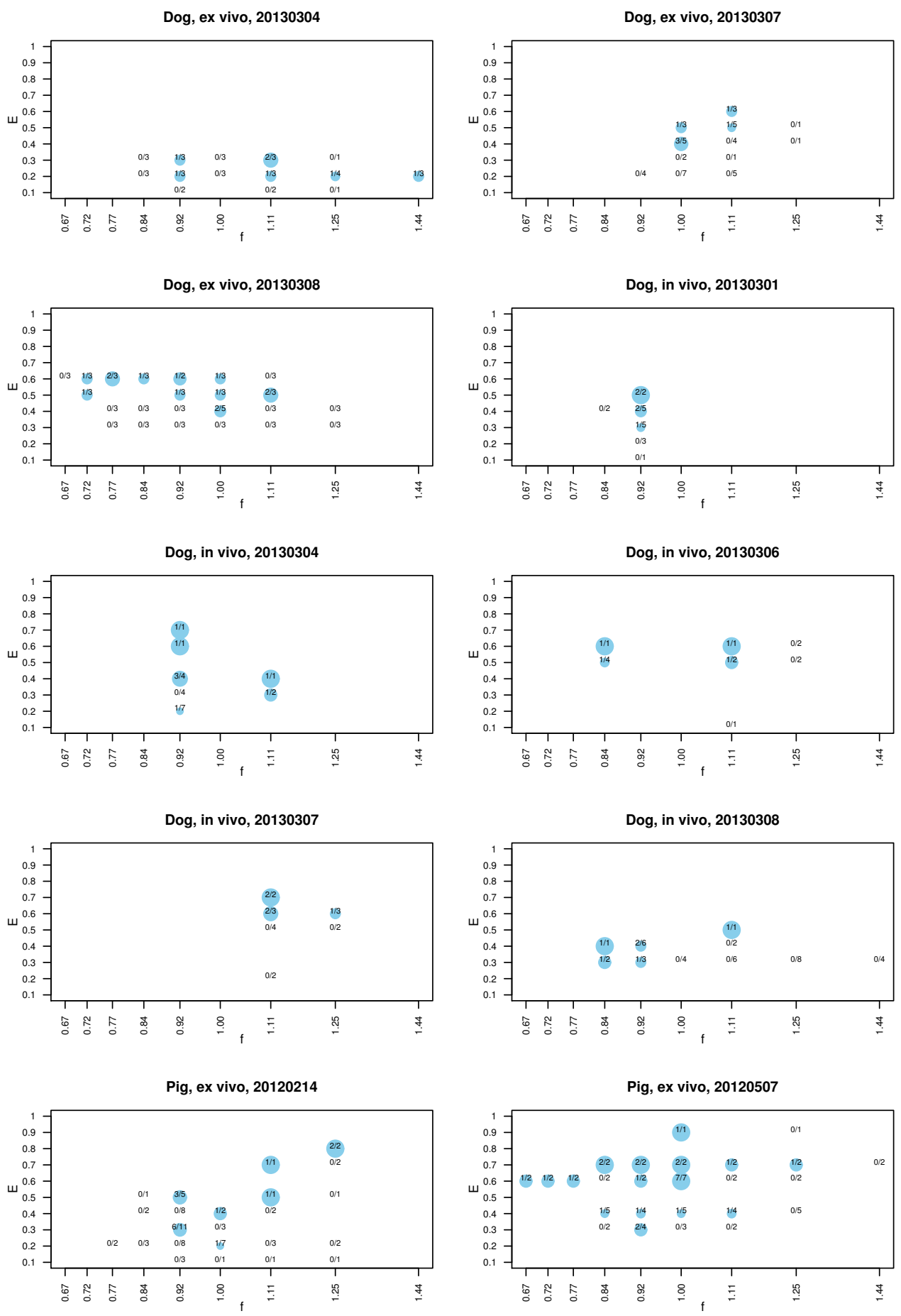

FiguRE B.14: LEAP Success for different pacing frequencies 

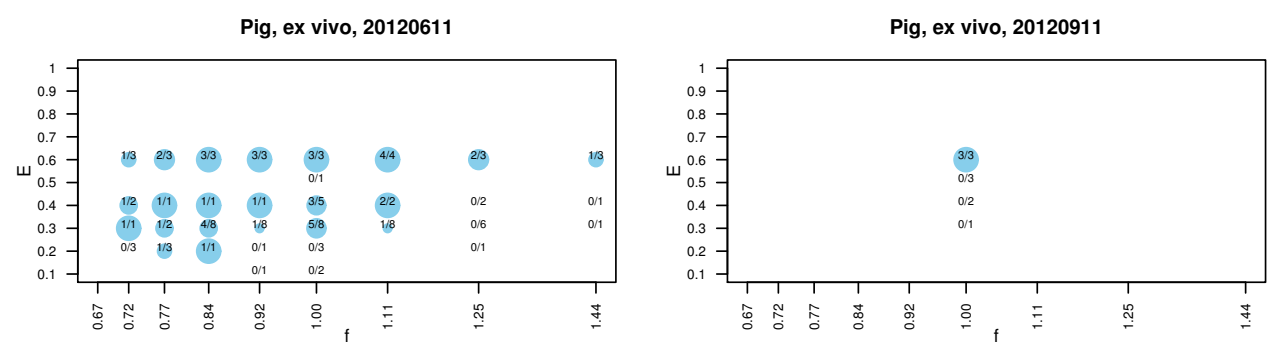

Pig, ex vivo, 20120912

Pig, ex vivo, 20121208
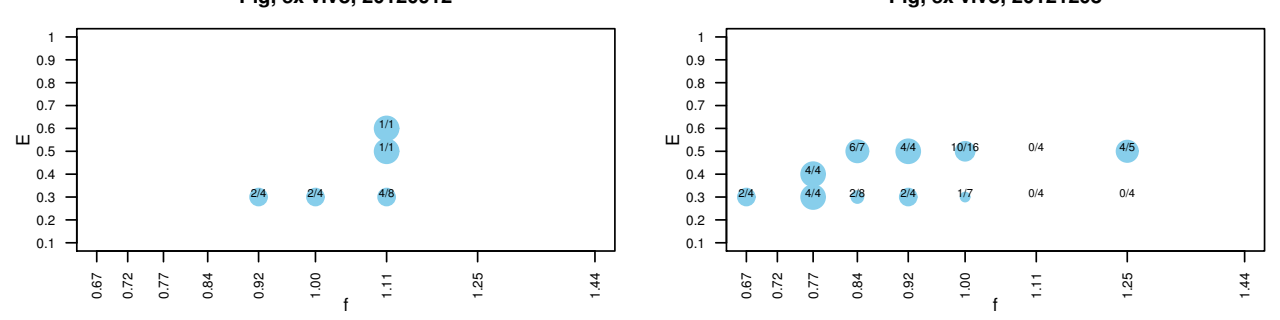

Pig, ex vivo, 20121209
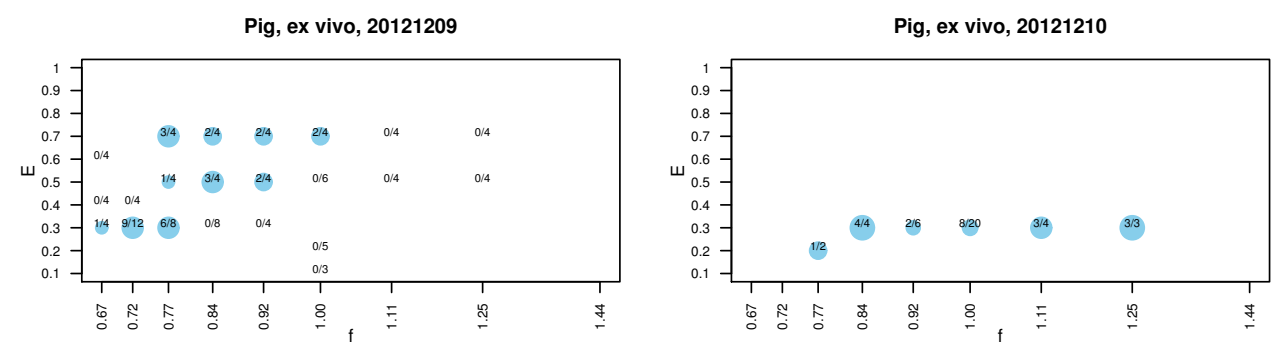

Pig, in vivo, 20110926

Pig, in vivo, 20111212
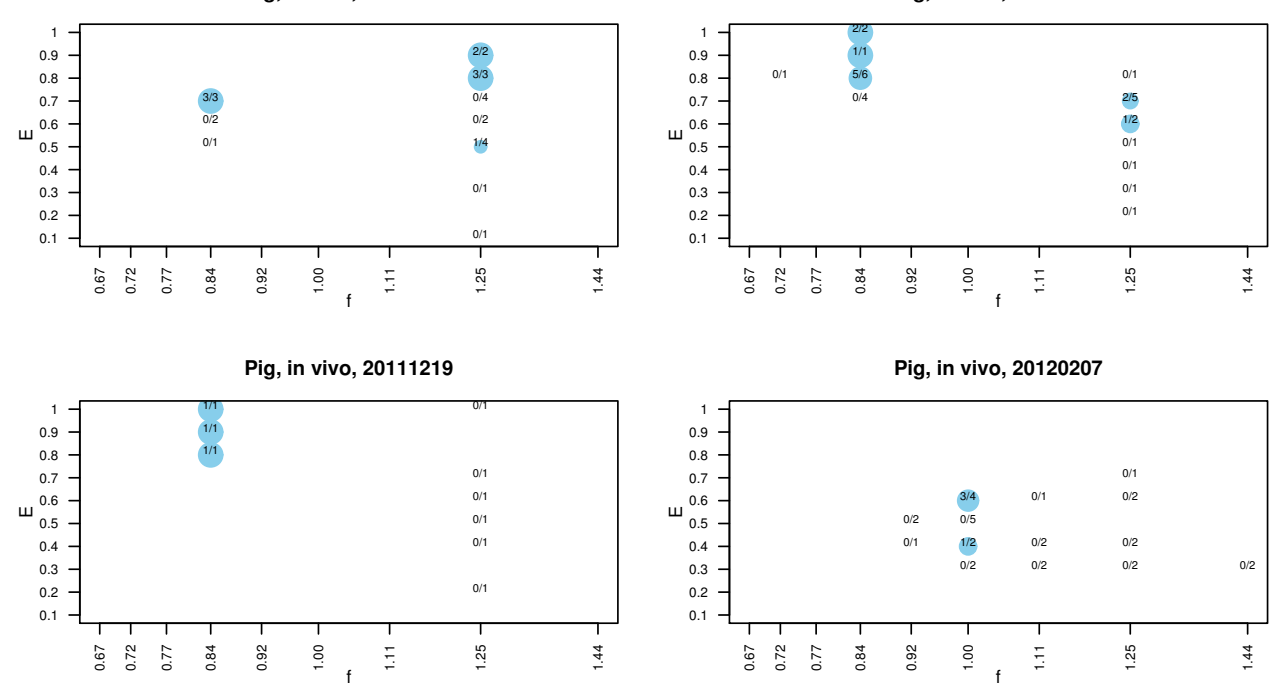

FiguRE B.15: LEAP Success for different pacing frequencies 
Pig, in vivo, 20120213

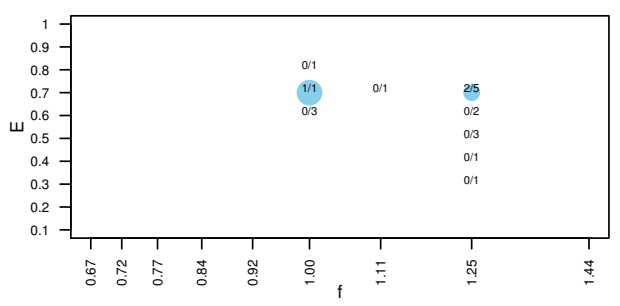

Pig, in vivo, 20120507

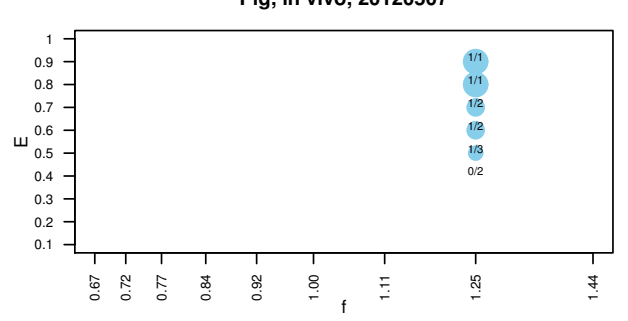

Pig, in vivo, 20120521

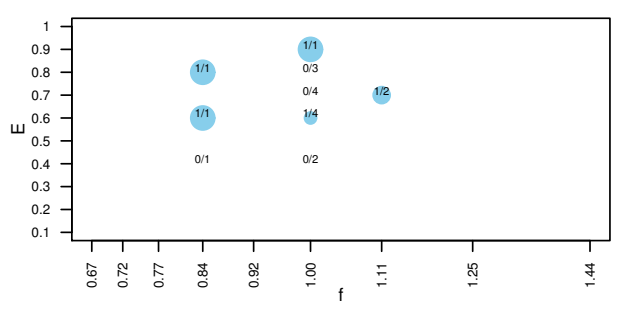

Pig, in vivo, 20120214

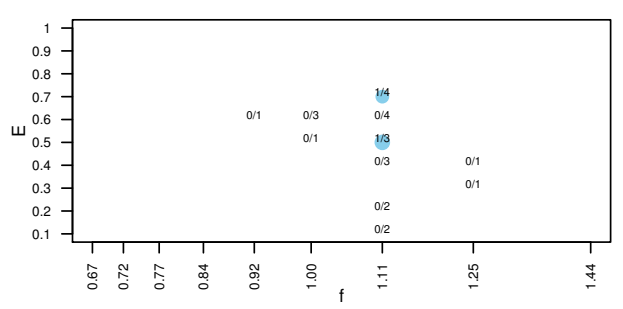

Pig, in vivo, 20120514

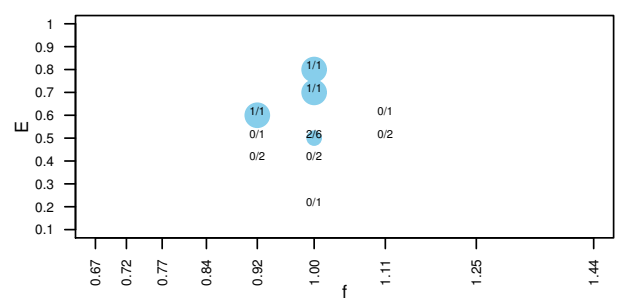

Pig, in vivo, 20120611

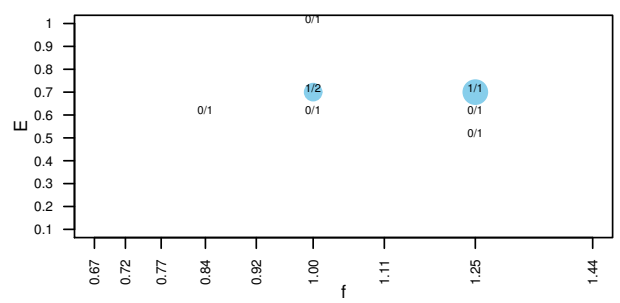

FIGURE B.16: LEAP Success for different pacing frequencies 



\section{Bibliography}

[1] Yaneer Bar-Yam. Dynamics of complex systems. Addison-Wesley, 1997. ISBN 9780201557480 .

[2] Freddy K. Lippert, Violetta Raffay, Marios Georgiou, Petter A. Steen, and Leo Bossaert. European resuscitation council guidelines for resuscitation 2010 section 10. the ethics of resuscitation and end-of-life decisions. Resuscitation, 81(10):14451451, October 2010. ISSN 0300-9572. doi: 10.1016/j.resuscitation.2010.08.013.

[3] Dirk Müller, Rahul Agrawal, and Hans-Richard Arntz. How sudden is sudden cardiac death? Circulation, 114(11):1146-1150, September 2006. ISSN 0009-7322, 1524-4539. doi: 10.1161/CIRCULATIONAHA.106.616318.

[4] Sumeet S. Chugh, Kyndaron Reinier, Carmen Teodorescu, Audrey Evanado, Elizabeth Kehr, Mershed Al Samara, Ronald Mariani, Karen Gunson, and Jonathan Jui. Epidemiology of sudden cardiac death: Clinical and research implications. Progress in Cardiovascular Diseases, 51(3):213-228, November 2008. ISSN 00330620. doi: $10.1016 /$ j.pcad.2008.06.003.

[5] Yash Lokhandwala, Gopi Krishna Panicker, and Shantanu Deshpande. Sudden cardiac death-an indian perspective. CVD Prevention and Control, 4(2):103-108, June 2009. ISSN 1875-4570. doi: 10.1016/j.cvdpc.2009.02.004.

[6] B. Hygriv Rao, B. K. S. Sastry, Sumeet S. Chugh, Sharada Kalavakolanu, Johann Christopher, Deepika Shangula, Radhika Korabathina, and P. Krishnam Raju. Contribution of sudden cardiac death to total mortality in india - a population based study. International Journal of Cardiology, 154(2):163-167, January 2012. ISSN 0167-5273. doi: 10.1016/j.ijcard.2010.09.016.

[7] Flavio Fenton and Elizabeth Cherry. Models of cardiac cell. Scholarpedia, 3(8): 1868, 2008. ISSN 1941-6016. doi: 10.4249/scholarpedia.1868.

[8] Richard FitzHugh. Impulses and physiological states in theoretical models of nerve membrane. Biophysical Journal, 1(6):445-466, July 1961. ISSN 0006-3495. doi: 10.1016/S0006-3495(61)86902-6. 
[9] Dwight Barkley. A model for fast computer simulation of waves in excitable media. Physica D: Nonlinear Phenomena, 49(1-2):61-70, April 1991. ISSN 0167-2789. doi: 10.1016/0167-2789(91)90194-E.

[10] Vladimir E. Bondarenko, Gyula P. Szigeti, Glenna C. L. Bett, Song-Jung Kim, and Randall L. Rasmusson. Computer model of action potential of mouse ventricular myocytes. American Journal of Physiology - Heart and Circulatory Physiology, 287(3):H1378 -H1403, 2004. doi: 10.1152/ajpheart.00185.2003.

[11] Arthur T. Winfree. The Geometry of Biological Time. Springer Science \& Business Media, 2001. ISBN 9780387989921.

[12] Vladimir Sergeevich Zykov. Simulation of Wave Processes in Excitable Media. Manchester University Press ND, 1987. ISBN 9780719024726.

[13] A. N. Zaikin and Anatol M. Zhabotinsky. Concentration wave propagation in two-dimensional liquid-phase self-oscillating system. Nature, 225(5232):535-537, February 1970. ISSN $\$$ ffooterJournalISSN\}. doi: 10.1038/225535b0.

[14] Richard J. Field, Endre Koros, and Richard M. Noyes. Oscillations in chemical systems. II. thorough analysis of temporal oscillation in the bromate-cerium-malonic acid system. Journal of the American Chemical Society, 94(25):8649-8664, 1972. ISSN 0002-7863. doi: 10.1021/ja00780a001.

[15] Arthur T. Winfree, Scott Caudle, Gang Chen, Patrick McGuire, and Zoltan Szilagyi. Quantitative optical tomography of chemical waves and their organizing centers. Chaos: An Interdisciplinary Journal of Nonlinear Science, 6(4):617-626, December 1996. ISSN 10541500. doi: doi:10.1063/1.166208.

[16] Patricia Dähmlow, Sergio Alonso, Markus Bär, and Marcus J. B. Hauser. Twists of opposite handedness on a scroll wave. Physical Review Letters, 110(23):234102, June 2013. doi: 10.1103/PhysRevLett.110.234102.

[17] Tomas Rokicki, Andrew Trevorrow, Tim Hutton, Dave Greene, Jason Summers, and Maks Verver. Golly. http://golly.sourceforge.net/, 2010.

[18] Norbert Wiener and Arturo Rosenblueth. The mathematical formulation of the problem of conduction of impulses in a network of connected excitable elements, specifically in cardiac muscle. Arch Inst Cardiol Mex, 16(3):205-265, July 1946.

[19] L. Reshodko and Jan Bureš. Computer simulation of reverberating spreading depression in a network of cell automata. Biological Cybernetics, 18(3):181-189, 1975. ISSN 0340-1200. doi: 10.1007/BF00326688. 
[20] Illés J. Farkas, Dirk Helbing, and Tamás Vicsek. Social behaviour: Mexican waves in an excitable medium. Nature, 419(6903):131-132, September 2002. ISSN 00280836. doi: 10.1038/419131a.

[21] Illés J. Farkas and Tamás Vicsek. Initiating a mexican wave: An instantaneous collective decision with both short- and long-range interactions. Physica A: Statistical Mechanics and its Applications, 369(2):830-840, September 2006. ISSN 0378-4371. doi: 10.1016/j.physa.2006.01.075.

[22] Peter Devreotes. Dictyostelium discoideum: a model system for cell-cell interactions in development. Science, 245(4922):1054-1058, September 1989. ISSN 0036-8075, 1095-9203. doi: 10.1126/science.2672337.

[23] John J. Tyson and James D. Murray. Cyclic AMP waves during aggregation of dictyostelium amoebae. Development, 106(3):421-426, July 1989. ISSN 0950-1991, 1477-9129.

[24] John J. Tyson, Kevin A. Alexander, V.S. Manoranjan, and James D. Murray. Spiral waves of cyclic amp in a model of slime mold aggregation. Physica D: Nonlinear Phenomena, 34(1-2):193-207, January 1989. ISSN 0167-2789. doi: 10.1016/0167-2789(89)90234-0.

[25] Robert Plonsey and Roger Barr. The four-electrode resistivity technique as applied to cardiac muscle. IEEE Transactions on Biomedical Engineering, BME-29(7):541 -546, July 1982. ISSN 0018-9294. doi: 10.1109/TBME.1982.324927.

[26] Markus Bär and Markus Eiswirth. Turbulence due to spiral breakup in a continuous excitable medium. Physical Review E, 48(3):R1635-R1637, 1993. doi: 10.1103/PhysRevE.48.R1635.

[27] Klaus Golenhofen. Physiologie heute: Lehrbuch, Kompendium, Fragen und Antworten ; mit 7 Tabellen. Urban und Fischer, München; Jena, 2000. ISBN 34374248079783437424809 .

[28] Miguel Valderrábano. Influence of anisotropic conduction properties in the propagation of the cardiac action potential. Progress in Biophysics and Molecular Biology, 94(1-2):144-168, May 2007. ISSN 0079-6107. doi: 10.1016/j.pbiomolbio. 2007.03.014.

[29] Gerhard A. Holzapfel and Ray W. Ogden. Constitutive modelling of passive myocardium: a structurally based framework for material characterization. Philosophical Transactions of the Royal Society A: Mathematical, Physical and Engineering Sciences, 367(1902):3445-3475, September 2009. ISSN 1364-503X, 14712962. doi: 10.1098/rsta.2009.0091. 
[30] Arthur C. Guyton and John E. Hall. Textbook of medical physiology. Saunders, Philadelphia, 10 edition, 2000. ISBN 0-7216-8677-X.

[31] Alain Karma. Physics of cardiac arrhythmogenesis. Annual Review of Condensed Matter Physics, 4(1):313-337, 2013. doi: 10.1146/ annurev-conmatphys-020911-125112.

[32] Tsu-Juey Wu, Shien-Fong Lin, Ali Baher, Zhilin Qu, Alan Garfinkel, James N. Weiss, Chih-Tai Ting, and Peng-Sheng Chen. Mother rotors and the mechanisms of d600-induced type 2 ventricular fibrillation. Circulation, 110(15):2110-2118, October 2004. ISSN 0009-7322, 1524-4539. doi: 10.1161/01.CIR.0000143834.51102.91.

[33] Eric A. Sobie, Robert C. Susil, and Leslie Tung. A generalized activating function for predicting virtual electrodes in cardiac tissue. Biophysical journal, 73(3):1410$1423,1997$.

[34] Leslie Tung. The generalized activating function. In Igor R. Efimov, Mark W. Kroll, and Patrick J. Tchou, editors, Cardiac Bioelectric Therapy, pages 111-132. Springer US, 2009. ISBN 978-0-387-79403-7.

[35] Matthew G. Fishler. Syncytial heterogeneity as a mechanism underlying cardiac far-field stimulation during defibrillation-level shocks. Journal of Cardiovascular Electrophysiology, 9(4):384-394, 1998. ISSN 1540-8167. doi: 10.1111/j.1540-8167. 1998.tb00926.x.

[36] Gregory P. Walgott, Stephen B. Knisley, Xiaohong Zhou, Jonathan G. Newton, and Raymond E. Ideker. On the mechanism of ventricular defibrillation. Pacing and Clinical Electrophysiology, 20(2):422-431, 1997. ISSN 1540-8159. doi: 10. 1111/j.1540-8159.1997.tb06201.x.

[37] Gregory P. Walcott, Cheryl R. Killingsworth, and Raymond E. Ideker. Do clinically relevant transthoracic defibrillation energies cause myocardial damage and dysfunction? Resuscitation, 59(1):59-70, October 2003. ISSN 0300-9572. doi: 10.1016/S0300-9572(03)00161-8.

[38] Eleanor B. Schron, Derek V. Exner, Qing Yao, Louise S. Jenkins, Jonathan S. Steinberg, James R. Cook, Steven P. Kutalek, Peter L. Friedman, Rosemary S. Bubien, Richard L. Page, and Judy Powell. Quality of life in the antiarrhythmics versus implantable defibrillators trial impact of therapy and influence of adverse symptoms and defibrillator shocks. Circulation, 105(5):589-594, February 2002. ISSN 0009-7322, 1524-4539. doi: 10.1161/hc0502.103330. 
[39] Samuel F. Sears and Jamie B. Conti. Quality of life and psychological functioning of icd patients. Heart, 87(5):488-493, May 2002. ISSN , 1468-201X. doi: 10.1136/ heart.87.5.488.

[40] Helena C. M. Kamphuis, Rob de Leeuw, Richard Derksen, Richard N. W. Hauer, and Jaques a. M. Winnubst. Implantable cardioverter defibrillator recipients: quality of life in recipients with and without ICD shock delivery a prospective study. Europace, 5(4):381-389, October 2003. ISSN 1099-5129, 1532-2092. doi: 10.1016/S1099-5129(03)00078-3.

[41] Michael O. Sweeney, Mark S. Wathen, Kent Volosin, Ismaile Abdalla, Paul J. DeGroot, Mary F. Otterness, and Alice J. Stark. Appropriate and inappropriate ventricular therapies, quality of life, and mortality among primary and secondary prevention implantable cardioverter defibrillator patients results from the pacing fast VT REduces shock ThErapies (PainFREE rx II) trial. Circulation, 111(22):2898-2905, June 2005. ISSN 0009-7322, 1524-4539. doi: 10.1161/CIRCULATIONAHA.104.526673.

[42] Maha Ahmad, Lauren Bloomstein, Marc Roelke, Alan D. Bernstein, and Victor Parsonnet. Patients' attitudes toward implanted defibrillator shocks. Pacing and Clinical Electrophysiology, 23(6):934-938, 2000. ISSN 1540-8159. doi: 10.1111/j. 1540-8159.2000.tb00877.x.

[43] Valentin Krinsky, Frédéric Plaza, and V. Voignier. Quenching a rotating vortex in an excitable medium. Physical Review E, 52(3):2458-2462, September 1995. doi: 10.1103/PhysRevE.52.2458.

[44] Alain Pumir, Sitabhra Sinha, S. Sridhar, Médéric Argentina, Marcel Hörning, Simonetta Filippi, Christian Cherubini, Stefan Luther, and Valentin Krinsky. Wavetrain-induced termination of weakly anchored vortices in excitable media. Physical Review E, 81(1):010901, January 2010. doi: 10.1103/PhysRevE.81.010901.

[45] Stefan Luther, Flavio H. Fenton, Bruce G. Kornreich, Amgad Squires, Philip Bittihn, Daniel Hornung, Markus Zabel, James Flanders, Andrea Gladuli, Luis Campoy, Elizabeth M. Cherry, Gisa Luther, Gerd Hasenfuss, Valentin I. Krinsky, Alain Pumir, Robert F. Gilmour, and Eberhard Bodenschatz. Low-energy control of electrical turbulence in the heart. Nature, 475(7355):235-239, July 2011. ISSN 0028-0836. doi: 10.1038/nature10216.

[46] Tariq Baig and Philip Bittihn. Personal communication, 2013. 
[47] Alain Pumir and Valentin Krinsky. Unpinning of a rotating wave in cardiac muscle by an electric field. Journal of Theoretical Biology, 199(3):311-319, August 1999. ISSN 0022-5193. doi: 10.1006/jtbi.1999.0957.

[48] Philip Bittihn, Amgad Squires, Gisa Luther, Eberhard Bodenschatz, Valentin Krinsky, Ulrich Parlitz, and Stefan Luther. Phase-resolved analysis of the susceptibility of pinned spiral waves to far-field pacing in a two-dimensional model of excitable media. Philosophical Transactions of the Royal Society A: Mathematical, Physical and Engineering Sciences, 368(1918):2221-2236, May 2010. ISSN 1364-503X, 1471-2962. doi: 10.1098/rsta.2010.0038.

[49] Anna Behrend, Philip Bittihn, and Stefan Luther. Predicting unpinning success rates for a pinned spiral in an excitable medium. In Computing in Cardiology, 2010, pages 345-348, September 2010.

[50] Philip Bittihn, Marcel Hörning, and Stefan Luther. Negative curvature boundaries as wave emitting sites for the control of biological excitable media. Physical Review Letters, 109(11):118106, September 2012. doi: 10.1103/PhysRevLett.109.118106.

[51] Benjamin E. Steinberg, Leon Glass, Alvin Shrier, and Gil Bub. The role of heterogeneities and intercellular coupling in wave propagation in cardiac tissue. Philosophical Transactions of the Royal Society A: Mathematical, Physical and Engineering Sciences, 364(1842):1299-1311, May 2006. ISSN 1364-503X, 1471-2962. doi: $10.1098 /$ rsta.2006.1771.

[52] Gil Bub, Alvin Shrier, and Leon Glass. Spiral wave generation in heterogeneous excitable media. Physical Review Letters, 88(5):058101, January 2002. doi: 10. 1103/PhysRevLett.88.058101.

[53] Fagen Xie, Zhilin Qu, Alan Garfinkel, and James N. Weiss. Electrophysiological heterogeneity and stability of reentry in simulated cardiac tissue. American Journal of Physiology - Heart and Circulatory Physiology, 280(2):H535-H545, February 2001. ISSN 0363-6135, 1522-1539.

[54] Michael Vinson, Arkady Pertsov, and José Jalife. Anchoring of vortex filaments in 3d excitable media. Physica D: Nonlinear Phenomena, 72(1-2):119-134, April 1994. ISSN 0167-2789. doi: 10.1016/0167-2789(94)90171-6.

[55] Zulma A. Jiménez and Oliver Steinbock. Scroll wave filaments self-wrap around unexcitable heterogeneities. Physical Review E, 86(3):036205, September 2012. doi: 10.1103/PhysRevE.86.036205. 
[56] Christian Mewis, Ioakim Spyridopoulos, and Reimer Riessen. Kardiologie compact: Alles für Station und Facharztprüfung. Georg Thieme Verlag, February 2006. ISBN 9783131576620 .

[57] Paul A. Iaizzo. Handbook of cardiac anatomy, physiology, and devices. Springer, New York, NY, 2009. ISBN 978160327371816032737199781603273725 1603273727 .

[58] Cecil D. Murray. The physiological principle of minimum work applied to the angle of branching of arteries. The Journal of General Physiology, 9(6):835-841, July 1926. ISSN 0022-1295.

[59] Cecil D. Murray. The physiological principle of minimum work. Proceedings of the National Academy of Sciences of the United States of America, 12(3):207-214, March 1926. ISSN 0027-8424.

[60] Geoffrey B. West, James H. Brown, and Brian J. Enquist. A general model for the origin of allometric scaling laws in biology. Science, 276(5309):122-126, April 1997. doi: 10.1126/science.276.5309.122.

[61] Yifang Zhou, Ghassan S. Kassab, and Sabee Molloi. On the design of the coronary arterial tree: a generalization of murray's law. Physics in Medicine and Biology, 44(12):2929-2945, 1999. ISSN 0031-9155.

[62] Ghassan S. Kassab. Scaling laws of vascular trees: of form and function. Am J Physiol Heart Circ Physiol, 290(2):H894-903, February 2006. doi: 10.1152/ ajpheart.00579.2005.

[63] Walter R. Stahl. Scaling of respiratory variables in mammals. Journal of Applied Physiology, 22(3):453-460, March 1967. ISSN 8750-7587, 1522-1601.

[64] N.A. Edwards. Scaling of renal functions in mammals. Comparative Biochemistry and Physiology Part A: Physiology, 52(1):63-66, 1975. ISSN 0300-9629. doi: 10. 1016/S0300-9629(75)80128-9.

[65] Ghassan S. Kassab, Carmela A. Rider, Nina J. Tang, and Yuan-Cheng C. Fung. Morphometry of pig coronary arterial trees. American Journal of Physiology Heart and Circulatory Physiology, 265(1):H350-H365, July 1993. ISSN 0363-6135, 1522-1539.

[66] NIST US Department of Commerce. NIST: X-ray mass attenuation coefficients, July 2004. URL http://www.nist.gov/pml/data/xraycoef/index.cfm. NIST: X-Ray Mass Attenuation Coefficients. 
[67] NIST US Department of Commerce. NIST: X-ray mass attenuation coefficients - table 3, July 2004. URL http://physics.nist.gov/PhysRefData/ XrayMassCoef/tab3.html.

[68] J. H. Hubbell and Stephen M. Seltzer. NIST: X-ray mass attenuation coefficients - table 4, 1996. URL http://physics.nist.gov/PhysRefData/XrayMassCoef/ tab4.html

[69] David R. Lide and H.P.R. Frederikse, editors. CRC Handbook of Chemistry and Physics. CRC Press Inc, 75th edition, June 1994. ISBN 0-8493-0475-X.

[70] Jay Tepperman and David Pearlman. Effects of exercise and anemia on coronary arteries of small animals as revealed by the corrosion-cast technique. Circulation Research, 9(3):576-584, May 1961. ISSN 0009-7330, 1524-4571. doi: 10.1161/01. RES.9.3.576.

[71] Morris E. Weaver, George A. Pantely, J. David Bristow, and Herbert D. Ladley. A quantitative study of the anatomy and distribution of coronary arteries in swine in comparison with other animals and man. Cardiovascular Research, 20(12): 907-917, December 1986. ISSN 0008-6363,. doi: 10.1093/cvr/20.12.907.

[72] Xavier Tizon, Qingfen Lin, Tomas Hansen, Gunilla Borgefors, Lars Johansson, Håkan Ahlström, and Hans Frimmel. Identification of the main arterial branches by whole-body contrast-enhanced MRA in elderly subjects using limited user interaction and fast marching. Journal of Magnetic Resonance Imaging: JMRI, 25 (4):806-814, April 2007. ISSN 1053-1807. doi: 10.1002/jmri.20848.

[73] Daniel Hornung and Stefan Luther. Automatic coronary vessel reconstruction from CT images. Biomedizinische Technik/Biomedical Engineering, 55(s1):115118, October 2010. ISSN 1862-278X. doi: 10.1515/BMT.2010.701.

[74] Johannes Schindelin, Ignacio Arganda-Carreras, Erwin Frise, Verena Kaynig, Mark Longair, Tobias Pietzsch, Stephan Preibisch, Curtis Rueden, Stephan Saalfeld, Benjamin Schmid, Jean-Yves Tinevez, Daniel James White, Volker Hartenstein, Kevin Eliceiri, Pavel Tomancak, and Albert Cardona. Fiji: an opensource platform for biological-image analysis. Nature Methods, 9(7):676-682, 2012. ISSN 1548-7091. doi: 10.1038/nmeth.2019.

[75] Caroline A. Schneider, Wayne S. Rasband, and Kevin W. Eliceiri. NIH image to ImageJ: 25 years of image analysis. Nature Methods, 9(7):671-675, 2012. ISSN 1548-7091. doi: 10.1038/nmeth.2089.

[76] Apache commons math, 2010. URL https://commons.apache.org/proper/ commons-math/. 
[77] Ta-Chih Lee, Rangasami L. Kashyap, and Chong-Nam Chu. Building skeleton models via 3-d medial surface axis thinning algorithms. CVGIP: Graphical Models and Image Processing, 56(6):462-478, November 1994. ISSN 1049-9652. doi: 10. 1006/cgip.1994.1042.

[78] Ignacio Arganda-Carreras. Skeletonize3d, 2008. URL http://fiji.sc/ Skeletonize3D

[79] Ignacio Arganda-Carreras. AnalyzeSkeleton, 2009. URL http://fiji.sc/ AnalyzeSkeleton.

[80] Wilhelm Burger and Mark Burge. Digital image processing an algorithmic introduction using Java. Springer, New York, 2008. ISBN 97818462896821846289688.

[81] Silke Grabherr, Andreas Hess, Marek Karolczak, Michael J. Thali, Sebastian D. Friess, Willi A. Kalender, Richard Dirnhofer, and Valentin Djonov. Angiofilmediated visualization of the vascular system by microcomputed tomography: A feasibility study. Microscopy Research and Technique, 71(7):551-556, 2008. ISSN 1097-0029. doi: 10.1002/jemt.20585.

[82] Philip Bittihn. Complex Structure and Dynamics of the Heart. PhD thesis, Universität Göttingen, October 2013.

[83] Dirk Durrer, Rudolf Th. van Dam, G. E. Freud, Michiel J. Janse, Frits L. Meijler, and Robert C. Arzbaecher. Total excitation of the isolated human heart. Circulation, 41(6):899-912, June 1970. ISSN 0009-7322, 1524-4539. doi: 10.1161/01.CIR.41.6.899.

[84] Jean Provost, Viatcheslav Gurev, Natalia Trayanova, and Elisa E. Konofagou. Mapping of cardiac electrical activation with electromechanical wave imaging: An in silico-in vivo reciprocity study. Heart Rhythm, 8(5):752-759, May 2011. ISSN 1547-5271. doi: 10.1016/j.hrthm.2010.12.034.

[85] K.R. Gee, K.A. Brown, W-N.U. Chen, J. Bishop-Stewart, D. Gray, and I. Johnson. Chemical and physiological characterization of fluo-4 ca2+-indicator dyes. Cell Calcium, 27(2):97-106, February 2000. ISSN 0143-4160. doi: 10.1054/ceca.1999. 0095 .

[86] Vladimir G. Fast. Simultaneous optical imaging of membrane potential and intracellular calcium. Journal of Electrocardiology, 38(4, Supplement):107-112, October 2005. ISSN 0022-0736. doi: 10.1016/j.jelectrocard.2005.06.023.

[87] Eric Fluhler, Valerie G. Burnham, and Leslie M. Loew. Spectra, membrane binding, and potentiometric responses of new charge shift probes. Biochemistry, 24 (21):5749-5755, October 1985. ISSN 0006-2960. doi: 10.1021/bi00342a010. 
[88] Leslie M. Loew, Lawrence B. Cohen, James Dix, Eric N. Fluhler, Valerie Montana, Guy Salama, and Wu Jian-young. A naphthyl analog of the aminostyryl pyridinium class of potentiometric membrane dyes shows consistent sensitivity in a variety of tissue, cell, and model membrane preparations. The Journal of Membrane Biology, 130(1):1-10, October 1992. ISSN 0022-2631, 1432-1424. doi: 10.1007/BF00233734.

[89] Andy Yen Hsin Chen. Development and characterization of fiber-based systems for biomedical imaging. Thesis, ResearchSpace@Auckland, 2011. URL https: //researchspace.auckland.ac.nz/handle/2292/13361

[90] Evgeny Stambulchik, Paul J Turner, et al. Grace: a WYSIWYG 2D plotting tool for the X Window System and M*tif. http://plasma-gate.weizmann.ac.il/ Grace/, 2008. version 5.1.22-9ubuntu1.

[91] Charles D. Deakin, Jerry P. Nolan, Kjetil Sunde, and Rudolph W. Koster. European resuscitation council guidelines for resuscitation 2010 section 3. electrical therapies: Automated external defibrillators, defibrillation, cardioversion and pacing. Resuscitation, 81(10):1293-1304, October 2010. ISSN 0300-9572. doi: 10.1016/j.resuscitation.2010.08.008.

[92] Harry G. Mond and Alessandro Proclemer. The 11th world survey of cardiac pacing and implantable cardioverter-defibrillators: Calendar year 2009-a world society of arrhythmia's project. Pacing and Clinical Electrophysiology, 34(8):10131027, 2011. ISSN 1540-8159. doi: 10.1111/j.1540-8159.2011.03150.x.

[93] Alain Pumir, Vladimir P. Nikolski, Marcel Hörning, Akihiro Isomura, Konstantin Agladze, Kenichi Yoshikawa, Robert Gilmour, Eberhard Bodenschatz, and Valentin Krinsky. Wave emission from heterogeneities opens a way to controlling chaos in the heart. Physical review letters, 99(20):208101, 2007.

[94] Amgad Squires. Wave Emission From Heterogeneities For Low-Energy Termination Of Cardiac Arrhythmias. PhD thesis, Cornell University, January 2011. URL http://ecommons.library.cornell.edu/handle/1813/33488.

[95] Matthew C. Strain and Henry S. Greenside. Size-dependent transition to highdimensional chaotic dynamics in a two-dimensional excitable medium. Physical Review Letters, 80(11):2306-2309, March 1998. doi: 10.1103/PhysRevLett.80. 2306 .

[96] Richard J. Kovacs and John C. Bailey. Effects of acetylcholine on action potential characteristics of atrial and ventricular myocardium after bilateral cervical vagotomy in the cat. Circulation Research, 56(4):613-620, April 1985. ISSN 0009-7330, 1524-4571. doi: 10.1161/01.RES.56.4.613. 
[97] Claudia Richter. Cardiomyocyten im Chaos: makroskopische Untersuchungen kardialer Arrhythmien in-vitro unter dem Einfluss elektrischer Pulsfolgen und Parameteränderungen. PhD thesis, Universität Rostock, June 2010.

[98] Simon J. Crick, Mary N. Sheppard, Siew Yen Ho, and Robert H. Anderson. Localisation and quantitation of autonomic innervation in the porcine heart i: conduction system. Journal of Anatomy, 195(3):341-357, 1999. ISSN 1469-7580. doi: 10.1046/j.1469-7580.1999.19530341.x.

[99] Alexander V. Panfilov. Is heart size a factor in ventricular fibrillation? or how close are rabbit and human hearts? Heart Rhythm, 3(7):862-864, 2006. doi: 10.1016/j.hrthm.2005.12.022.

[100] Mark W. Kroll and Michael H. Lehmann, editors. Implantable Cardioverter Defibrillator Therapy: The Engineering-Clinical Interface. Springer, 1996. ISBN 978-1-4615-6345-7.

[101] Rudolph W. Koster, Paul Dorian, Fred W. Chapman, Paul W. Schmitt, Sharon G. O'Grady, and Robert G. Walker. A randomized trial comparing monophasic and biphasic waveform shocks for external cardioversion of atrial fibrillation. American Heart Journal, 147(5):e1-e7, May 2004. ISSN 0002-8703. doi: 10.1016/j.ahj.2003. 10.049 .

[102] James W. Leitch, Anne M. Gillis, D. George Wyse, Raymond Yee, George J. Klein, Gerard Guiraudon, Robert S. Sheldon, Henry J. Duff, Teresa M. Kieser, and L. Brent Mitchell. Reduction in defibrillator shocks with an implantable device combining antitachycardia pacing and shock therapy. Journal of the American College of Cardiology, 18(1):145-151, July 1991. ISSN 0735-1097. doi: 10.1016/ S0735-1097(10)80232-3.

[103] Mark S. Wathen, Michael O. Sweeney, Paul J. DeGroot, Alice J. Stark, Jodi L. Koehler, Michael B. Chisner, Christian Machado, and Wayne O. Adkisson. Shock reduction using antitachycardia pacing for spontaneous rapid ventricular tachycardia in patients with coronary artery disease. Circulation, 104(7):796-801, August 2001. ISSN 0009-7322, 1524-4539. doi: 10.1161/hc3101.093906.

[104] Mohammed Chebbok, Amgad Squires, Johannes Schroeder-Schetelig, Markus Zabel, Gerd Hasenfuss, Eberhard Bodenschatz, Flavio Fenton, and Stefan Luther. Low-energy anti-fibrillation pacing (LEAP): a gentle, non traumatic defibrillation option. European Heart Journal, 33(suppl 1):381, August 2012. ISSN 0195-668X, 1522-9645. doi: 10.1093/eurheartj/ehs282. 
[105] Nipon Chattipakorn, Isabelle Banville, Richard A. Gray, and Raymond E. Ideker. Mechanism of ventricular defibrillation for near-defibrillation threshold shocks a whole-heart optical mapping study in swine. Circulation, 104(11):1313-1319, September 2001. ISSN 0009-7322, 1524-4539. doi: 10.1161/hc3601.094295.

[106] Peter J. A. Bollen, Axel Kornerup Hansen, and Aage Kristian Olsen Alstrup. The laboratory swine. The laboratory animal pocket reference series. CRC Press, Boca Raton, Fla. [u.a.], 2. ed. edition, 2010. ISBN 1-439-81528-3, 978-1-439-81528-1.

[107] Camelia Gabriel, Azadeh Peyman, and E. H. Grant. Electrical conductivity of tissue at frequencies below $1 \mathrm{MHz}$. Physics in Medicine and Biology, 54(16):48634878, August 2009. ISSN 0031-9155. doi: 10.1088/0031-9155/54/16/002.

[108] Nicolas Salihin, Falko Schlottig, Thomas Hefti, Henning Schliephake, and Rudolf Gruber. Primärstabilität von dentalimplantaten im polylaktidverstärkten knochen - eine pilotstudie am göttinger mini pig, May 2013. URL http://www.ag-kiefer.de/tagungen/aktuelle-jahrestagung-2013/ wissenschaftliches-programm/freitag-10052013/ landgraf-friedrich-saal-i/vortraege-tag-der-forschung-1.html.

[109] B B Roe, J C Hutchinson, N H Fishman, D J Ullyot, and D L Smith. Myocardial protection with cold, ischemic, potassium-induced cardioplegia. The Journal of thoracic and cardiovascular surgery, 73(3):366-374, March 1977. ISSN 0022-5223.

[110] Douglas L. Jones, William D. Irish, and George J. Klein. Defibrillation efficacy. comparison of defibrillation threshold versus dose-response curve determination. Circulation Research, 69(1):45-51, July 1991. ISSN 0009-7330, 1524-4571. doi: 10.1161/01.RES.69.1.45.

[111] Igor Singer and Doug Lang. Defibrillation threshold: Clinical utility and therapeutic implications. Pacing and Clinical Electrophysiology, 15(6):932-949, 1992. ISSN 1540-8159. doi: 10.1111/j.1540-8159.1992.tb03083.x.

[112] Charles D. Swerdlow, Andrea M. Russo, and Paul J. Degroot. The dilemma of ICD implant testing. Pacing and Clinical Electrophysiology, 30(5):675-700, 2007. ISSN 1540-8159. doi: 10.1111/j.1540-8159.2007.00730.x.

[113] S. Serge Barold, Bengt Herweg, and Anne B. Curtis. The defibrillation safety margin of patients receiving ICDs: A matter of definition. Pacing and Clinical Electrophysiology, 28(9):881-882, 2005. ISSN 1540-8159. doi: 10.1111/j.1540-8159. 2005.00191.x.

[114] Kenneth A. Ellenbogen and Mark A. Wood. Cardiac pacing and ICDs. Blackwell Pub., Chichester, UK; Hoboken, NJ, 2008. ISBN 140516350X 9781405163507. 
[115] Mark Kroll, Michael Lehmann, and Patrick J. Tchou. The defibrillation dosage. In Mark W. Kroll and Michael H. Lehmann, editors, Implantable Cardioverter Defibrillator Therapy: The Engineering-Clinical Interface, number 188 in Developments in Cardiovascular Medicine, pages 63-88. Springer US, January 1996. ISBN 978-1-4613-7914-0, 978-1-4615-6345-7.

[116] Igor Singer and Douglas Lang. The defibrillation threshold. In Mark W. Kroll and Michael H. Lehmann MD, editors, Implantable Cardioverter Defibrillator Therapy: The Engineering-Clinical Interface, number 188 in Developments in Cardiovascular Medicine, pages 89-129. Springer US, January 1996. ISBN 978-1-4613-7914-0, 9781-4615-6345-7.

[117] C. F. Babbs, S. J. Whistler, and G. K. Yim. Temporal stability and precision of ventricular defibrillation threshold data. American Journal of Physiology - Heart and Circulatory Physiology, 235(5):H553-H558, November 1978.

[118] Richard E. Kerber, Natesa G. Pandian, Robert Hoyt, Susan R. Jensen, Samon Koyanagi, Joseph Grayzel, and Robert Kieso. Effect of ischemia, hypertrophy, hypoxia, acidosis, and alkalosis on canine defibrillation. American Journal of Physiology - Heart and Circulatory Physiology, 244(6):H825-H831, June 1983. ISSN 0363-6135, 1522-1539.

[119] Eleftheria P. Tsagalou, Maria I. Anastasiou-Nana, Christos E. Charitos, Costantinos X. Siafakas, Stavros G. Drakos, Argirios Ntalianis, John V. Terrovitis, Emmanuel M. Mavrikakis, Antonios Doufas, and John N. Nanas. Time course of fibrillation and defibrillation thresholds after an intravenous bolus of amiodarone - an experimental study. Resuscitation, 61(1):83-89, April 2004. ISSN 0300-9572. doi: 10.1016/j.resuscitation.2003.12.003.

[120] Valentin Krinsky and Konstantin I. Agladze. Interaction of rotating waves in an active chemical medium. Physica D: Nonlinear Phenomena, 8(1-2):50-56, July 1983. ISSN 0167-2789. doi: 10.1016/0167-2789(83)90310-X.

[121] Stefan Luther. Personal communication. 2012.

[122] Sanjeev Saksena, The Pcd Investigators, and Participating Institutions. Defibrillation thresholds and perioperative mortality associated with endocardial and epicardial defihrillation lead systems. Pacing and Clinical Electrophysiology, 16 (1):202-207, 1993. ISSN 1540-8159. doi: 10.1111/j.1540-8159.1993.tb01562.x.

[123] Trevor J. Hastie, Robert John Tibshirani, and Jerome H. Friedman. The Elements of Statistical Learning: Data Mining, Inference, and Prediction. Springer series in statistics. Springer, New York, N.Y, 2nd edition, 2009. ISBN 978-0-387-84857-0. 



\section{Acknowledgments}

This work would not have been possible without the support and input of many friends and colleagues and combinations thereof, so I wish to thank them here.

I would like to thank my thesis committee, first of all my advisor, Stefan Luther, for the years I was allowed to spend in his group. Although up to his neck in work to assemble the research group, acquire new and reacquire old members, apply for and receive funding, hold exams and seminars, he always found time to do science and advise those who needed it. He guided me through my thesis project, with fresh ideas and and even practical hands-on help in the lab at midnight when important experiments would start the next day. The group would not be the same without him. Like Stefan a member of my thesis committee, Ulrich Parlitz brought with him what can probably best described as the spirit of the Third Institute of Physics at the University of Göttingen. Nearly a decade ago, it was him who kindled my love for nonlinear systems and for unexpected complexity, so I am glad I could be working with him. Many years of experience made him the man to ask for all kinds of numerical or data analytical problems. Last but not least, Eberhard Bodenschatz was also willing to join my thesis committee. As the only one not in Stefan Luther's research group, it was his to sometimes show me a different perspective on my own work. With his insightful questions and remarks he provided me with the necessary input to focus on the important steps towards completion of my thesis.

Jörg Enderlein was kindly willing to review this work and to be on my examination committee. Since cardiac dynamics is not his primary field of interest, I wish to thank him all the more for accepting this duty. The same goes for the other members of my examination committee, Eleni Katifori and Andreas Janshoff who will thankfully spend one afternoon to hear and ask about this work.

New ideas and great results can never come from a single group alone. Flavio Fenton brought many unconventional ways of thinking, of coding and of nearly everything else. It was always as much fun as it was productive to be with him, whether on his many visits to Europe and Göttingen or in Cornell in his former lab, and it was certainly some 
of the best time. Of course, I will not omit "our" emeritus, Valentin Krinsky. He not only is one of the great scientific minds in our group, but also had his very own way of hinting people into the right direction. His aversion against giving answers, but instead loving to ask the right questions, was infamous, but in the end, he was right on most things.

I also wish to thank my colleagues for the great time I had with them and for all the fruitful discussions about science, Club Mate and everything under the sun. In the context of this work, my special thanks go to Philip and Tariq for proof-reading this thesis. With Philip and Johannes, I had very competent and thoughtful partners in the preparation and execution of the experiments. Marion, Claudia and Mohammed were always there in the labs and experiments when needed and showed me many practical things no theoretical physicist would ever think of in their wildest dreams.

The experiments would not have been possible without many other people. Foremost, Laura and Niels in Cornell provided us with everything we needed, from chemicals over solder to meals late at night. Robert Gilmour lent not just his name to the Cornell lab, we sometimes had the feeling that his presence alone was enough to make devices and methods alike just work. Of course, we never could have built our "shock box" defibrillator ourselves, so Karl Lautscham also deserves a honorable mention.

Last, but of course not the least, I wish to thank my girlfriend Miriam for the time past, the present and the future. I could not hope for a better partner!

\section{Funding}

The research leading to these results has received funding from the European Community's Seventh Framework Programme FP7/2007-2013 under grant agreement No. HEALTH-F2-2009-241526, EUTrigTreat and from the German Ministry for Education and Research (FKZ 01EZ0905/6).

This research has received funding from the SFB 1002 "Modulatorische Einheiten bei Herzinsuffizienz". 